\title{
Renormalization-Group Theory for Quantum Dissipative Systems in Nonequilibrium
}

\author{
Dissertation \\ zur Erlangung des Doktorgrades \\ der Mathematisch-Naturwissenschaftlichen Fakultäten \\ der Georg-August-Universität zu Göttingen
}

\author{
vorgelegt von \\ Markus Keil \\ aus Groß-Gerau
}

Göttingen 2001 
D7

Referent: Prof. Dr. Kurt Schönhammer

Korreferent: Prof. Dr. Herbert Schoeller

Tag der mündlichen Prüfung: 29.01.2002 


\section{Contents}

1 Introduction $\quad 3$

2 The polaron $\quad 7$

2.1 Fröhlich Hamiltonian . . . . . . . . . . . . . . . . . . . 8

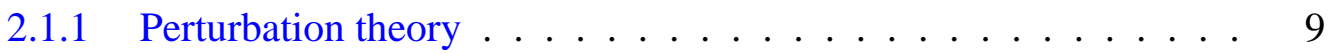

2.1.2 Method of Lee, Low and Pines . . . . . . . . . . . . . . . . . 10

2.1.3 Feynman's method .................................... 11

2.2 Flow equations . . . . . . . . . . . . . . . . . . . . . . 14

2.3 Real-time renormalization-group for the polaron . . . . . . . . . . . . . . 19

2.4 Discussion . . . . . . . . . . . . . . . . 26

3 The spin-boson model $\quad 29$

3.1 Model Hamiltonian . . . . . . . . . . . . . . . . . . . . . . 30

3.2 Real-time renormalization-group formalism . . . . . . . . . . . . 33

3.2.1 Kinetic equation . . . . . . . . . . . . . . . 34

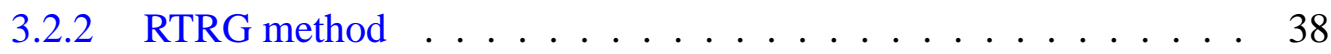

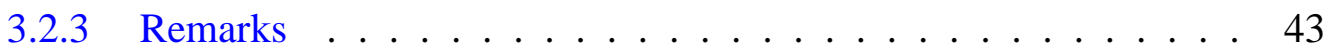

3.2.4 Equilibrium correlation functions . . . . . . . . . . . . 45

3.3 RG equations for the spin-boson model . . . . . . . . . . . . . . 48

3.3 .1 Initial values . . . . . . . . . . . . . . . . . . 48

3.3.2 The $t_{c}$ dependence of the bath contraction . . . . . . . . . . 49

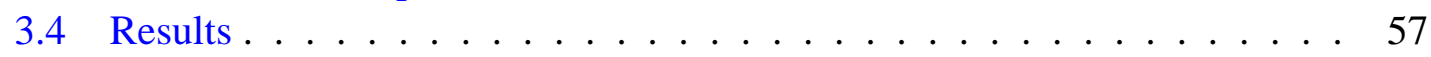

3.4.1 The renormalized tunnel matrix element . . . . . . . . . . 57

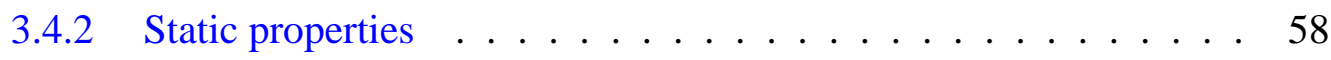

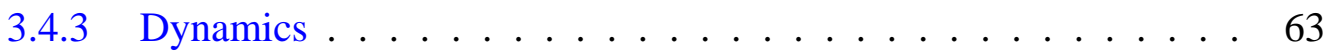

3.4 The Shiba-relation . . . . . . . . . . . . 70

3.5 Discussion ............................... 74

4 Coupled quantum dots $\quad \mathbf{7 7}$

4.1 Model Hamiltonian . . . . . . . . . . . . . . . . . . . . 78

4.2 The tunnel current within the RTRG . . . . . . . . . . . . . . 83

4.2.1 Exact expression for the stationary tunnel current . . . . . . . 83

4.2.2 RG equations for the coupled quantum dots . . . . . . . . 85 
4.3 Results .............................. 92

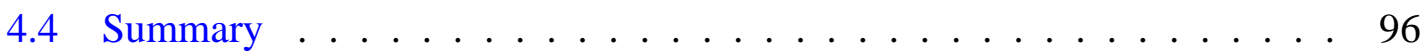

5 Two-lead Kondo model 97

5.1 Model Hamiltonian . . . . . . . . . . . . . . . . . . . . . . . . . 98

5.2 The RTRG for the two-lead Kondo model . . . . . . . . . . . . . . . . . 99

5.3 The running couplings . . . . . . . . . . . . . . . . . . . . . . . . 104

5.3.1 The role of the external voltage . . . . . . . . . . . . . 104

5.3.2 Exact results . . . . . . . . . . . . . . . 105

5.4 Discussion . . . . . . . . . . . . . . . . . 110

6 Conclusion $\quad 111$

$\begin{array}{lll}\text { A The noninteracting blip approximation } & 115\end{array}$

B Relation of the SBM to the Kondo model 119

$\begin{array}{lll}\text { C Double vertices in the RTRG } & 121\end{array}$

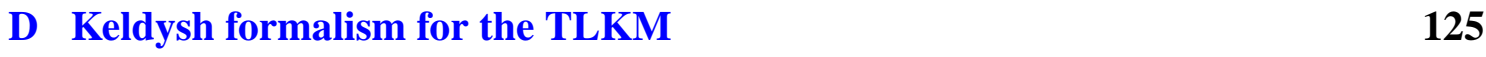

E Numerical methods $\quad 129$

E.1 Ordinary differential equations . . . . . . . . . . . . . . . . . 129

E.2 Exponentials of a matrix in differential equations . . . . . . . . . 131

E.3 Fourier transforms . . . . . . . . . . . . . . . 135 


\section{Chapter 1}

\section{Introduction}

If a physical system is prepared in nonequilibrium, a coupling to an environment gives rise to dissipation. Thereby the system will finally attain a stationary state. Classically, the interaction term in the Hamilton function leads to dissipative forces in the equations of motion. In many cases one ends up with a generalized Langevin equation for the coordinates of the local system [1]. To obtain a correct description of such systems, however, one has to consider the problem quantum mechanically. This leads to quantum dissipative systems where dissipation is generated by the interaction term in the Hamilton operator which acts on the product Hilbert space of the local system and the environmental bath. Typically the environment is given by a particle bath (e.g. electron reservoir) or a bath of quasiparticles representing excitation modes (e.g. phonons in a solid). The dynamics is determined by the Heisenberg equation while the thermodynamic properties follow from the partition function. The local system is described by the reduced density matrix, which is the trace of the full density matrix over the bath degrees of freedom.

In case of a small coupling of the local system to the environment, such problems can be approximately solved by using standard perturbation theory. However, for larger coupling constants this treatment becomes insufficient and other methods have to be applied. One often uses the path-integral formulation of Feynman [2], where the interaction term may be accounted for by an influence functional. One then ends up with an effective theory for the local system's degrees of freedom which usually has to be solved approximately.

Any interacting system may also be studied by exactly diagonalizing the Hamiltonian. However, for quantum dissipative systems this is a complex problem due to the high dimension of the Hilbert space of the bath. Therefore, one may only apply some approximate diagonalization. A general method that serves this purpose is the flow equation formalism of Wegner [3]. In this method the unitary transformation which diagonalizes the Hamiltonian is decomposed. This results in a sequence of infinitesimal transformations, and its differential formulation then leads to flow equations. This approach has often been used to determine the matrix elements of the diagonalized Hamiltonian, such as the ground-state energy. From these one may also calculate spectral properties, such as equilibrium correlation functions. However, the calculation of the diagonalizing unitary 
transformation requires much more effort within this formalism. Therefore, one does not obtain a solution for the system's dynamics.

The method of the flow equations can be viewed as a renormalization-group (RG) technique. RG procedures use some kind of operation to transform a physical system into an equivalent one. Successive application of this operation generates a sequence of systems which define a semi-group with respect to this operation. The elements of the group can be labeled by a parameter $\mu$. The idea of RG methods is that, for some limit of $\mu$, e.g. $\mu \rightarrow \infty$, one deals with a trivial system. Today RG methods are often used tools which are successful in describing many physical problems beyond perturbation theory [3] - [11]. However, until recently RG methods could only be used to calculate equilibrium quantities. They could not describe nonequilibrium stationary states or provide the dynamics of quantum dissipative systems. A new approach which also allows for the description of the dynamics in arbitrary nonequilibrium situations is the real-time renormalization-group (RTRG) by Schoeller [12]. Within this approach one develops an effective theory for the local system by introducing normal ordering and applying Wick's theorem regarding the bath operators. One then has to account for nontrivial expressions, which involve time-dependent bath contractions. In the RTRG scheme one defines the real-time parameter $t_{c}$ as a critical time scale, which serves as a cutoff regarding the time arguments of the contractions that one takes into account. To leave the physical problem invariant one has to account for this cutoff by renormalized operators for the local system. By considering the step $t_{c} \rightarrow t_{c}+d t_{c}$ with an increment $d t_{c}$ one then obtains a set of differential equations with respect to $t_{c}$. This defines a renormalization-group flow for physical quantities, which now depend on the flow parameter $t_{c}$. Within the RTRG one starts at $t_{c}=0$ with the original problem, and ends in the limit $t_{c} \rightarrow \infty$, where all contractions have been integrated out. In contrast to Poor Man's scaling equations [4], this approach takes the full time evolution into account. Thus, all time scales are considered and one generally does not need any further cutoff regarding the $t_{c}$ flow. Another advantage of this method is that one may work on the Keldysh contour. Thereby, nonHamiltonian dynamics is considered, so that both dynamical and stationary quantities of quantum dissipative systems can be calculated for any nonequilibrium problem.

The RTRG has been applied to equilibrium problems [13] and has been used to study nonequilibrium stationary states [14]. In this thesis we use the RTRG to analyze different quantum dissipative systems. These RG studies lead to results which could not be obtained before for these systems, such as nonequilibrium stationary states or the dynamics of the reduced density matrix. In fact, we present the first RG calculation of the timedependent reduced density matrix of a quantum dissipative system at all. We discuss the power and performance of the RTRG and present some further modifications. This will also convey an insight into the general properties and possibilities of this RG method.

As a starting point and a first application of the new RTRG method we consider the polaron problem described by the Fröhlich Hamiltonian [15] in the next chapter. It is a standard model for an electron interacting with phonons in a solid and has attracted much interest in the past 50 years. More recently also low-dimensional systems have often been studied [16] - [20]. We present approximate results for the ground-state energy as well as 
for the effective mass of the one-dimensional polaron in the regime of small to intermediate couplings for the case of vanishing temperature. For this analysis we do not have to work on the Keldysh contour, but it is sufficient to consider the $S$ matrix within the RTRG approach. We also generalize the standard methods of Lee, Low and Pines [21, 22] and that of Feynman [23] to the one-dimensional case with finite bandwidth. These approximations are then compared to the results following from the flow equation formalism and those obtained by the RTRG [24]. Although the comparisons show that the RTRG method is in principle able to describe the polaron beyond perturbation theory, we achieve only a low accuracy for this model. The difficulties of the RTRG concerning the polaron problem are discussed. Furthermore, it turns out that a nonequilibrium study of the polaron requires too much numerical effort.

In Chapter 3, the central part of this thesis, we study the spin-boson model using the RTRG method. In this case the problems which arise for the polaron model are not present. In contrast, here we use the RTRG formalism on the Keldysh contour to determine the full time evolution of the reduced density matrix starting from an arbitrary nonequilibrium state [25, 26]. Furthermore, we also calculate static quantities as well as equilibrium correlation functions. Our results are obtained for arbitrary parameters with the only restriction of not too large couplings, and they exhibit a remarkably high accuracy. The spin-boson model is a basic, yet nontrivial quantum dissipative model, which can be used for any physical or chemical system, where a local two-state system is coupled to a bosonic environment. Altough it has been examined very often (see Refs. [1, 27] and references therein), an exact solution of the spin-boson model is only known for special parameter values.

After we have given a detailed explanation of the general formalism of the RTRG, we discuss the explicit form of the differential equations for the spin-boson model. Considering the resulting RG equations we analytically find the correct renormalized energy scale of the problem. We solve the RG equations numerically to present results for the stationary reduced density matrix, the static spin susceptibility and the time-dependent reduced density matrix. For the latter we also determine both the oscillation frequency of the diagonal elements and the decay constants in the asymptotic regime, the dephasing and the relaxation time. Eventually, we use the RTRG to also calculate equilibrium correlation functions. The presented results show the correct scaling behaviour and are consistent with both QMC simulations and the Shiba-relation. Additionally, we draw comparisons to the noninteracting blip approximation (NIBA) [27] and to results obtained for the anisotropic Kondo model [28, 29].

A more complicated quantum dissipative system is considered in Chapter 4, where we study a system consisting of two coupled quantum dots in a phonon bath. This structure has been realized in a recent experiment [30], where the authors applied an external voltage to the double dot and measured the stationary tunnel current through it. Today quantum dot systems are often studied, as they can be used as realizations for many physical phenomena [31, 32, 33]. In the experiment described in Ref. [30] the influence of the phonon environment on the stationary tunnel current was examined at low temperature. We study this out-of-equilibrium problem using the RTRG, by which again the whole pa- 
rameter space with small to moderately large couplings to the environment is accessible. Since the coupled quantum dots in a phonon bath are closely related to the spin-boson model, we can make use of the insights we have gained in the preceding chapter. In contrast to a previous qualitative theoretical analysis [34] our approach yields quantitatively reliable results. Furthermore, within our analysis we also allow for an additional electronphonon interaction term, which arises from the finite extension of the electron densities within the dots. Comparing our results with the experiment we show that one has to account for the variation of this finite extension with the applied bias between the quantum dots. Using the experimental data we calculate the width of the electron densities as a function of the energy difference between the dots [35].

In the preceding chapters we have neglected "double vertex objects", which have not been present in the original Hamiltonians, but are generated during the renormalizationgroup procedure. This approximation led to the restriction in the coupling constants. In contrast, in Chapter 5 we study the two-lead Kondo model, where a Kondo impurity is coupled to two bands. Since the original Kondo Hamiltonian already contains such double vertex objects, the previously used approximation is not applicable then. However, within the usual RTRG method double vertex objects give rise to retardation effects, which lead to substantially more complicated RG equations. We avoid this problem by introducing a formulation of the RTRG in energy space. The Kondo model has attracted much, both theoretical and experimental, interest (for a review, see [36]). Recently, especially the case of the two-lead Kondo model with a finite external voltage has been studied [37] [42]. Based on qualitative arguments, a two-channel regime for the running couplings was proposed for that model [37]. However, the authors excluded the effects of rates which may destroy the two-channel physics [43]. Applying the RTRG to this model we restrict ourselves to the analysis of an effective Hamiltonian, thus we also disregard the effects of rates which can only be accounted for by studying the problem on the Keldysh contour. Thereby we quantitatively find the two-channel behaviour of the running couplings which confirms the proposal of Ref. [37]. 


\section{Chapter 2}

\section{The polaron}

The polaron has often been examined since Fröhlich proposed the corresponding Hamiltonian [15] (for a review, see Ref. [44]). It serves as a standard model for various problems involving a nonrelativistic particle moving in a scalar field, e.g. the interaction between nucleons and scalar mesons or a single electron in a solid interacting with phonons. The physical picture is that the particle polarizes the environment and must drag this polarization with it, which affects its energy and effective mass. The problem has been studied by Lee, Low and Pines, who used a variational method [21, 22]. They obtained results for small to intermediate coupling strengths. For large couplings the polaron has been examined by Pekar et al. [45, 46]. Feynman provided an analysis for arbitrary couplings [23]. Since polaron effects have been observed in low-dimensional systems, the problem has also been studied in two dimensions [16, 17, 18] and in one dimension [19, 20].

We examine the one-dimensional polaron problem. It can be realized e.g. for a Bloch electron in a one-dimensional wire or macromolecular structure. The excitation of an electron is strongly influenced by the interaction with longitudinal optical phonons [47]. If the conduction band is partially filled, one can linearize the electronic spectrum and the model is exactly solvable by using bosonization techniques [20]. However, if the conduction band is empty, it is necessary to consider a quadratic spectrum for the electron with a bare mass $m_{0}$. This leads to the one-dimensional Fröhlich Hamiltonian with a constant coupling to the phonons. It is our purpose to examine the ground-state energy and the effective mass of the electron for this problem at vanishing temperature using renormalization-group methods. We apply the flow equations introduced by Wegner $[3,48]$ and the real-time renormalization-group (RTRG) technique recently developed by Schoeller [12,13]. Furthermore, we compare our results with first and second order perturbation theory, with Feynman's variational principle, and with the method of Lee, Low and Pines generalized to the one-dimensional case with finite bandwidth. Regarding the flow equations the approximation used in Ref. [49] turns out to be insufficient to describe renormalization effects of the polaron. Using the RTRG for not too large coupling constants we find a ground-state energy below the one following from Feynman's method and perturbation theory of second order and a value for the effective mass between the result of Feynman and the one of Lee, Low and Pines [24]. However, due to a further 
approximation within the RTRG only a low accuracy is achieved.

\subsection{Fröhlich Hamiltonian}

A Hamiltonian for the polaron system was introduced by Fröhlich in 1954 [15]. He considered a single electron in a polar medium, which interacts with the polarization field resulting from displacements of the ions. As only the longitudinal waves enter the interaction term one can write for the quantized polarization at the position $\vec{x}$

$$
\vec{P}(\vec{x})=\frac{1}{\sqrt{V}} \sum_{\vec{q}}\left[\frac{\omega}{8 \pi}\left(\frac{1}{\epsilon_{\infty}}-\frac{1}{\epsilon}\right)\right]^{\frac{1}{2}} \frac{\vec{q}}{|\vec{q}|} e^{i \vec{q} \vec{x}}\left(a_{\vec{q}}-a_{-\vec{q}}^{\dagger}\right)
$$

where we assume periodic boundary conditions. $a_{\vec{q}}^{\dagger}\left(a_{\vec{q}}\right)$ creates (annihilates) a longitudinal optical phonon with the wave vector $\vec{q}$, where $\vec{q}$ lies in the first Brioullin zone. These phonons are assumed to be dispersionless: $\omega_{\vec{q}}=\omega$. The solid has the volume $V$, the static dielectric constant is $\epsilon$, and the one for large frequencies is $\epsilon_{\infty}$. The operator for the charge density of the electron is given by

$$
\rho(\vec{x})=e \psi^{\dagger}(\vec{x}) \psi(\vec{x}) .
$$

$\psi^{\dagger}(\vec{x})(\psi(\vec{x}))$ is the creation (annihilation) operator of an electron at position $\vec{x}$. It can be expressed as

$$
\psi^{\dagger}(\vec{x})=\frac{1}{\sqrt{V}} \sum_{\vec{k}} c_{\vec{k}}^{\dagger} e^{-i \vec{k} \vec{x}} \quad\left(\psi(\vec{x})=\frac{1}{\sqrt{V}} \sum_{\vec{k}} c_{\vec{k}} e^{i \vec{k} \vec{x}}\right)
$$

where $c_{\vec{k}}^{\dagger}\left(c_{\vec{k}}\right)$ creates (annihilates) an electron with wave vector $\vec{k}$. The Hamiltonian then follows from the classical Hamilton function. It consists of a free part for the electron, $H_{0}$, one for the phonons, $H_{B}$, and an interaction part $H_{V}$ :

$$
H=H_{0}+H_{B}+H_{V}
$$

They are given by

$$
\begin{aligned}
H_{0} & =\sum_{k} \epsilon_{k} c_{k}^{\dagger} c_{k}, \\
H_{B} & =\sum_{q} \hbar \omega a_{q}^{\dagger} a_{q}, \\
H_{V} & =\sum_{k, q}\left(M_{q} a_{-q}^{\dagger}+M_{-q}^{*} a_{q}\right) c_{k+q}^{\dagger} c_{k} .
\end{aligned}
$$

Now the indices $k, q$ denote the corresponding wave vectors $\vec{k}, \vec{q}$, the spin index is omitted, as it is conserved under the interaction. We consider a slowly moving electron so 
that only large wavelengths are important. In this picture the electron energy $\epsilon_{k}$ can be assumed as $(\hbar|\vec{k}|)^{2} / 2 m_{0}, m_{0}$ being the bare mass of the electron. Consequently, the first Brioullin zone for the one-dimensional case is given by the bandwidth $D$ as $\left[-\sqrt{2 m_{0} D} / \hbar, \sqrt{2 m_{0} D} / \hbar\left[\right.\right.$. While the electron-phonon interaction coefficients $M_{q}$ are proportional to $1 /|\vec{q}|$ in case of the bulk polaron, the one-dimensional situation involves a $q$ independent coefficient $M$ [18]. We define

$$
M=\hbar \omega \frac{\sqrt{2 \alpha}}{\sqrt{L \sqrt{2 m_{0} \omega / \hbar}}}
$$

where $L$ is the one-dimensional normalization volume. In analogy to the three-dimensional case $\alpha$ is a dimensionless coupling constant. In the following we discuss some earlier approximate solutions of the polaron problem and generalize them to the one-dimensional case with finite bandwidth $D$. Henceforth we choose units such that $\hbar=m_{0}=\omega=1$.

\subsubsection{Perturbation theory}

The simplest approximation that can be applied to the problem is standard perturbation theory in the coupling constant $\alpha$. One obtains for the energy $E_{k}$ of the polaron with momentum $k$

$$
\begin{aligned}
E_{k}= & \frac{k^{2}}{2}-\sum_{q} \frac{M^{2}}{\Delta_{k, q}} \\
& -\frac{1}{2} \sum_{q, q^{\prime}} M^{4} \frac{\left(\Delta_{k, q}+\Delta_{k, q^{\prime}}\right)\left(\Delta_{k, q}+\Delta_{k, q^{\prime}}-\Gamma_{k, q, q^{\prime}}\right)}{\Delta_{k, q}^{2} \Delta_{k, q^{\prime}}^{2} \Gamma_{k, q, q^{\prime}}}+\mathcal{O}\left(\alpha^{3}\right),
\end{aligned}
$$

where we defined the energies

$$
\begin{aligned}
\Delta_{k, q} & =\epsilon_{k+q}-\epsilon_{k}+1 \\
\Gamma_{k, q, q^{\prime}} & =\epsilon_{k+q+q^{\prime}}-\epsilon_{k}+2 .
\end{aligned}
$$

Note that for $|k|<\sqrt{2}$ the fractions are well defined. In the continuum limit the $q$ sums correspond to integrals which can be performed analytically in first order in $\alpha$. With the bandwidth $D$ one obtains for the ground-state energy

$$
E_{0}=-\alpha \frac{2}{\pi} \arctan (\sqrt{D})+\mathcal{O}\left(\alpha^{2}\right)
$$

The inverse effective mass $1 / m$ is given as $1 / m=\left.\frac{d^{2}}{d k^{2}} E_{k}\right|_{k=0}$, thus

$$
\frac{1}{m}=1-\frac{\alpha}{\pi}\left(\arctan (\sqrt{D})+\frac{\sqrt{D}}{D+1}-\frac{2 \sqrt{D}}{(D+1)^{2}}\right)+\mathcal{O}\left(\alpha^{2}\right)
$$




\subsubsection{Method of Lee, Low and Pines}

The method of Lee, Low and Pines is based on the usual variational principle [21, 22]. In the following we will use their approach to derive results for the ground-state energy $E_{0}$ and the effective mass $m$ for the one-dimensional polaron with finite bandwidth $D$. Assuming that successive virtual phonons are emitted independently they chose the following ansatz with the parameter $v_{k, q}$ for the ground-state of the polaron with momentum $k$ :

$$
\begin{aligned}
\left|\psi_{k}\right\rangle^{L L P}\left(v_{k, q}\right)=\int_{L} d x \frac{1}{\sqrt{L}} & \exp \left(i x\left(k-\sum_{q} q a_{q}^{\dagger} a_{q}\right)\right) \psi^{\dagger}(x) \\
& \times N \exp \left(\sum_{q} v_{k, q} a_{-q}^{\dagger}\right)|0\rangle .
\end{aligned}
$$

The normalization factor $N$ is given by

$$
N=\exp \left(-\frac{1}{2} \sum_{q} v_{k, q}^{2}\right)
$$

This yields for the energy $E_{k}$

$$
E_{k}^{L L P}=\frac{k^{2}}{2}+2 \sum_{q} M v_{k, q}+\frac{1}{2}\left(\sum_{q} v_{k, q}^{2} q\right)^{2}+\sum_{q} v_{k, q}^{2} \Delta_{k, q} .
$$

Minimizing the energy with respect to the coefficients $v_{k, q}$ leads to

$$
v_{k, q} \Delta_{k, q}+M+v_{k, q} q \sum_{q^{\prime}} v_{k, q^{\prime}}^{2} q^{\prime}=0
$$

Following Lee, Low and Pines [21] we choose the ansatz

$$
\eta_{k} k=-\sum_{q} v_{k, q}^{2} q
$$

which leads to

$$
v_{k, q}=-\frac{M}{\Delta_{k\left(1-\eta_{k}\right), q}} .
$$

We now assume

$$
0<\eta_{k}<1
$$

for all $k$, so that the above fraction in Eq. (2.15) is well defined for $|k|<\sqrt{2}$. Inserting this in Eq. (2.14) we integrate to get an expression for $\eta_{k}$ :

$$
\eta_{k}=\frac{\frac{\alpha}{\pi}\left(\arctan (\sqrt{D})+\frac{\sqrt{D}}{D+1}-\frac{2 \sqrt{D}}{(D+1)^{2}}\right)}{\frac{\alpha}{\pi}\left(\arctan (\sqrt{D})+\frac{\sqrt{D}}{D+1}-\frac{2 \sqrt{D}}{(D+1)^{2}}\right)+1}+\mathcal{O}\left(k^{2}\right)
$$


For the calculation of the ground-state energy and the effective mass it is sufficient to take into account only the lowest order in $k$ for $\eta_{k}$, so that Eq. (2.16) holds. It then follows from Eqs. (2.12) and (2.15):

$$
\begin{aligned}
E_{0}^{L L P}= & -\alpha \frac{2}{\pi} \arctan (\sqrt{D}) \\
\frac{1}{m^{L L P}}= & \frac{1}{1+\frac{\alpha}{2}} \\
& +\frac{\frac{\alpha}{2}}{\left(1+\frac{\alpha}{2}\right)} \cdot \frac{1-\frac{2}{\pi}\left(\arctan (\sqrt{D})+\frac{\sqrt{D}}{D+1}-\frac{2 \sqrt{D}}{(D+1)^{2}}\right)}{1+\frac{\alpha}{\pi}\left(\arctan (\sqrt{D})+\frac{\sqrt{D}}{D+1}-\frac{2 \sqrt{D}}{(D+1)^{2}}\right)} .
\end{aligned}
$$

These approximate results are valid for small to intermediate couplings $\alpha \lesssim 2$. One notices that the expression for the energy, Eq. (2.18), coincides with that of perturbation theory to first order of $\alpha$, Eq. (2.8). Thus perturbation theory of first order gives an upper bound for the ground-state energy.

\subsubsection{Feynman's method}

Feynman's variational approach [23] is able to treat both small to intermediate and strong couplings. Furthermore, for small couplings he could improve the approximate results of Lee, Low and Pines [21]. We apply Feyman's method to the one-dimensional polaron with a finite bandwidth and small to intermediate couplings. Feynman's variational principle is based on the path-integral formalism [2,50]. When the paths of the oscillators of the bath have been integrated out, one obtains for the effective Euclidean action

$$
S_{\mathrm{eff}}^{E}=\frac{1}{2} \int_{0}^{\beta} d \tau\left(\frac{d x}{d \tau}\right)^{2}-\frac{\alpha}{\sqrt{2}} \int_{0}^{\beta} d \tau \int_{0}^{\beta} d \tau^{\prime} e^{-\left|\tau-\tau^{\prime}\right|} \frac{1}{L} \sum_{q} e^{i q\left(x(\tau)-x\left(\tau^{\prime}\right)\right)},
$$

where we use the coordinate representation. One now considers the sum over all possible trajectories

$$
K=\int \mathcal{D} x(\tau) \exp \left(-S_{\text {eff }}^{E}\right) .
$$

Of course, $K$ will depend on $\beta$ as well as on the initial and final condition for the path $x(\tau)$. From the path-integral formalism we know that for $\beta \rightarrow \infty, K$ decays as $e^{-E_{0} \beta}$, $E_{0}$ being the ground-state energy. One now chooses an approximate effective action $\tilde{S}_{\text {eff }}^{E}$ which is more tractable than $S_{\text {eff. }}^{E}$. Let us consider the average of a functional $f(x(\tau))$ with the positive weight $\exp \left(-\tilde{S}_{\text {eff }}^{E}\right)$ :

$$
\langle f\rangle=\frac{\int \mathcal{D} x(\tau) \exp \left(-\tilde{S}_{\text {eff }}^{E}\right) f}{\int \mathcal{D} x(\tau) \exp \left(-\tilde{S}_{\text {eff }}^{E}\right)} .
$$

For any set of real quantities $g$ we then may use Jensen's inequality

$$
\langle\exp (g)\rangle \geq \exp (\langle g\rangle) \text {. }
$$


Thus

$$
K \geq \exp \left(\left\langle\tilde{S}_{\text {eff }}^{E}-S_{\text {eff }}^{E}\right\rangle\right) \int \mathcal{D} x(\tau) \exp \left(-\tilde{S}_{\text {eff }}^{E}\right)
$$

If we know the ground-state energy $\tilde{E}_{0}$ of the problem described by $\tilde{S}_{\text {eff }}^{E}$, this leads to an upper bound for $E_{0}$ :

$$
\tilde{E}_{0}+\lim _{\beta \rightarrow \infty} \frac{\left\langle S_{\text {eff }}^{E}-\tilde{S}_{\text {eff }}^{E}\right\rangle}{\beta} \geq E_{0}
$$

The left-hand side of the above equation may then be used as an approximate result for $E_{0}$. For small to intermediate couplings we choose Feynman's two-particle approximation with the free parameters $C$ and $w$ :

$$
\tilde{S}_{\text {eff }}^{E}=\frac{1}{2} \int_{0}^{\beta} d \tau\left(\frac{d x}{d \tau}\right)^{2}+\frac{1}{2} C \int_{0}^{\beta} d \tau \int_{0}^{\beta} d \tau^{\prime} e^{-w\left|\tau-\tau^{\prime}\right|}\left(x(\tau)-x\left(\tau^{\prime}\right)\right)^{2} .
$$

Following Feynman [23] one studies

$$
\begin{aligned}
I & =\left\langle\exp \left(i q\left(x(\tau)-x\left(\tau^{\prime}\right)\right)\right)\right\rangle \\
& =\frac{\int \mathcal{D} x(\tau) \exp \left(-\tilde{S}_{\text {eff }}^{E}+i q\left(x(\tau)-x\left(\tau^{\prime}\right)\right)\right)}{\int \mathcal{D} x(\tau) \exp \left(-\tilde{S}_{\text {eff }}^{E}\right)} .
\end{aligned}
$$

By considering the path, for which the exponent in Eq. (2.27) is maximum, he derived

$$
I=\exp \left(-\frac{2 C q^{2}}{v^{3} w}\left(1-e^{-v\left|\tau-\tau^{\prime}\right|}\right)-\frac{w^{2} q^{2}}{2 v^{2}}\left|\tau-\tau^{\prime}\right|\right)
$$

with the definition

$$
v^{2}=w^{2}+\frac{4 C}{w} .
$$

Expanding Eq. (2.28) with respect to $q$ up to order $q^{2}$ we obtain

$$
\left\langle\left(x(\tau)-x\left(\tau^{\prime}\right)\right)^{2}\right\rangle=\frac{4 C}{v^{3} w}\left(1-e^{-v\left|\tau-\tau^{\prime}\right|}\right)+\frac{w^{2}}{v^{2}}\left|\tau-\tau^{\prime}\right| .
$$

Then $\tilde{E}_{0}$ can be derived from the free action $(C=0)$ and the derivative

$$
\frac{d \tilde{E}_{0}}{d C}=\lim _{\beta \rightarrow \infty} \frac{1}{\beta} \int_{0}^{\beta} d \tau \int_{0}^{\beta} d \tau^{\prime} \frac{1}{2}\left\langle\left(x(\tau)-x\left(\tau^{\prime}\right)\right)^{2}\right\rangle e^{-w\left|\tau-\tau^{\prime}\right|} .
$$

This yields

$$
\begin{aligned}
\frac{d \tilde{E}_{0}}{d C} & =\frac{1}{v w} \\
\Longrightarrow \tilde{E}_{0} & =\frac{v-w}{2} .
\end{aligned}
$$


Furthermore, we obtain analogously to Ref. [23]

$$
\begin{aligned}
\lim _{\beta \rightarrow \infty} \frac{\left\langle S_{\text {eff }}^{E}-\tilde{S}_{\text {eff }}^{E}\right\rangle}{\beta}= & -\frac{\alpha \sqrt{2}}{\pi} \int_{0}^{\infty} d \tau e^{-\tau} \\
& \times \int_{0}^{\sqrt{2 D}} d q \exp \left(-\frac{2 C q^{2}}{v^{3} w}\left(1-e^{-v \tau}\right)-\frac{w^{2} q^{2}}{2 v^{2}} \tau\right) \\
& -\frac{C}{v w},
\end{aligned}
$$

where $D$ is again the bandwidth. Thus, the approximate ground-state energy $E_{0}^{F}$ reads

$$
\begin{aligned}
E_{0}^{F} & =\tilde{E}_{0}+\lim _{\beta \rightarrow \infty} \frac{\left\langle S_{\mathrm{eff}}^{E}-\tilde{S}_{\mathrm{eff}}^{E}\right\rangle}{\beta} \\
& =\frac{(v-w)^{2}}{4 v}-\alpha \frac{v}{\sqrt{\pi}} \int_{0}^{\infty} d \tau e^{-\tau}(g(\tau))^{-\frac{1}{2}} \operatorname{erf}\left(\frac{\sqrt{D g(\tau)}}{v}\right)
\end{aligned}
$$

with the error-function

$$
\operatorname{erf}(\mathrm{x})=\frac{2}{\sqrt{\pi}} \int_{0}^{x} d t e^{-t^{2}}
$$

and

$$
g(\tau)=\left(\frac{v^{2}-w^{2}}{v}\left(1-e^{-v \tau}\right)+w^{2} \tau\right) .
$$

The parameters $v$ and $w$ are chosen such that $E_{0}^{F}$ is minimum. For $v=w$ the result of perturbation theory of first order in $\alpha$ is reproduced. Following Feynman we treat small couplings by setting $v=(1+\delta) w$. Considering $\delta$ small one can now expand the right-hand side in Eq. (2.35) which yields

$$
\begin{aligned}
E_{0}^{F}= & \frac{1}{4} w \delta^{2}+\mathcal{O}\left(\delta^{3}\right) \\
& -\alpha\left(\frac{2}{\pi} \arctan \sqrt{D}+\delta \frac{2}{\pi}(\arctan \sqrt{D}-P)+\mathcal{O}\left(\delta^{2}\right)\right),
\end{aligned}
$$

where we introduced

$$
P=\frac{\sqrt{D}}{D+1}+\frac{2}{w}\left(\sqrt{w+1} \arctan \frac{\sqrt{D}}{\sqrt{w+1}}-\arctan \sqrt{D}\right)
$$

Minimizing the energy to this order we obtain

$$
\delta=\alpha \frac{4}{w \pi}(\arctan \sqrt{D}-P)
$$

Thus

$$
E_{0}^{F}=-\alpha \frac{2}{\pi} \arctan \sqrt{D}-\alpha^{2} \frac{4}{w \pi^{2}}(\arctan \sqrt{D}-P)^{2} .
$$


This expression is not sensitive to the choice of $w$. For $D \rightarrow \infty$ it is minimum for $w=3$, for $w=1$ the $\alpha^{2}$ coefficient drops by approximately $20 \%$. Therefore, we may choose $w=3$ for all $D$. This finally leads to

$$
\begin{aligned}
E_{0}^{F}= & -\alpha \frac{2}{\pi} \arctan \sqrt{D} \\
& -\alpha^{2} \frac{4}{3 \pi^{2}}\left(\frac{5}{3} \arctan \sqrt{D}-\frac{4}{3} \arctan \frac{\sqrt{D}}{2}-\frac{\sqrt{D}}{D+1}\right)^{2} .
\end{aligned}
$$

Within Feynman's approximation the effective mass $m$ can be calculated by considering paths with a final coordinate $x_{\beta}$. For small momenta, $K$ in Eq. (2.21) asymptotically decays as $e^{-E_{0} \beta-m x_{\beta}^{2} / 2 \beta}$, from which the mass can be determined. One sets $x_{\beta}=u \beta$, so that the value of $I$ in Eq. (2.28) now depends on $u$. Proceeding analogously as above one obtains

$$
\begin{aligned}
& m^{F}=1+\alpha \frac{1}{\pi}\left(\arctan \sqrt{D}+\frac{\sqrt{D}(D-1)}{(D+1)^{2}}\right) \\
&+\alpha^{2} \frac{4}{3 \pi^{2}}\left(\frac{5}{3} \arctan \sqrt{D}-\frac{4}{3} \arctan \frac{\sqrt{D}}{2}-\frac{\sqrt{D}}{D+1}\right) \\
& \times\left(\arctan \sqrt{D}+\arctan \frac{\sqrt{D}}{2}\right. \\
&\left.\quad+\frac{3 \sqrt{D}\left(D^{2}+5 D-2\right)}{(D+1)^{2}(D+4)}-\frac{36 \sqrt{D}^{3}(D+3)}{(D+1)^{2}(D+4)^{2}}\right),
\end{aligned}
$$

where we chose the same values of the parameters as for determining the ground-state energy. Since Eq. (2.40) follows from a variational principle Feynman's method gives an upper bound for the ground-state energy. In fact, it leads to more accurate results for the energy than for the mass (for quantitative studies concerning the accuracy in three dimensions see Ref. [51]). For $D \rightarrow \infty$ our results coincide with Refs. [18, 19].

\subsection{Flow equations}

The flow equations introduced by Wegner [3] are based on infinitesimal transformations which are successively applied to diagonalize the Hamiltonian. Formally one application of such an infinitesimal transformation is established by incrementing a continuous parameter $l$ by $d l$. This leads to a $l$ dependence of the transformed Hamiltonian $H(l)$. It may be written as

$$
H(l)=U(l) H U^{\dagger}(l)
$$

with a $l$ dependent unitary transformation $U(l)$. By convention one starts at $l=0$ :

$$
U(0)=\mathbb{1} \Longrightarrow H(0)=H .
$$


The infinitesimal transformations are now defined by a generator $\eta(l)$ :

$$
\eta(l)=\frac{d U(l)}{d l} U^{\dagger}(l)
$$

Thus, the resulting flow equation can formally be expressed by the commutator $[\cdot, \cdot]_{-}$:

$$
\frac{d}{d l} H(l)=[\eta(l), H(l)]_{-} .
$$

One useful choice for $\eta(l)$ is

$$
\eta(l)=\left[H_{d}(l), H_{r}(l)\right]_{-},
$$

where $H_{d}$ denotes the diagonal part of the Hamiltonian and $H_{r}$ the off-diagonal part. As a consequence of this choice the off-diagonal elements decay for $l \rightarrow \infty$. This can easily be shown if there are no degeneracies. However, even in the presence of degeneracies more detailed studies $[49,52,48]$ suggest the decay of $H_{r}$. With this choice of $\eta$ the problem arises that the formal flow equation, Eq. (2.45), does not correspond to a closed set of differential equations for the matrix elements of the Hamiltonian. This is due to the generation of new interaction terms when performing the commutators. Therefore, one usually has to truncate the system of flow equations.

Applying this scheme to the polaron one chooses for $\eta(l)$

$$
\eta(l)=\left[H_{0}(l)+H_{B}(l), H_{V}(l)\right]_{-}
$$

with $H_{0}(0)=H_{0}, H_{B}(0)=H_{B}$ and $H_{V}(0)=H_{V}$. The generated set of differential equations is truncated as in Ref. [49], where this approximation proved sufficient to explain the effective interaction between electrons. By using this truncation we neglect double phonon processes, thus $H_{0}(l), H_{B}(l)$ and $H_{V}(l)$ are given as in Eqs. (2.1) - (2.3), but with $l$ dependent coefficients $\epsilon_{k}(l), \omega(l)$ and $M_{k, q}(l)$. In general, we expect this approximation to be valid at least for small couplings $\alpha$. In the thermodynamic limit the phonon frequencies are not renormalized: $\omega(l)=\omega=$ const.. The flow equations read

$$
\begin{aligned}
\frac{d}{d l} \epsilon_{k}(l) & =-\sum_{q} 2 \Delta_{k, q}(l) M_{k, q}^{2}(l), \\
\frac{d}{d l} M_{k, q}(l) & =-\Delta_{k, q}^{2}(l) M_{k, q}(l),
\end{aligned}
$$

where we again used the definition in Eq. (2.6). Note that a $(k, q)$ dependence of the couplings $M_{k, q}$ is generated in the $l$ flow. When we examined the three-dimensional polaron using this formalism [48], the ground-state was in the focus of interest. Here, we are only interested in the ground-state energy and the effective mass of the (onedimensional) polaron. These quantities can directly be derived by integrating Eqs. (2.48) and (2.49), as the renormalized energies are given by $E_{k}=\lim _{l \rightarrow \infty} \epsilon_{k}(l)$. Eqs. (2.48) and (2.49) can be solved approximately [48]. If we, for example, neglect the $l$ dependence 


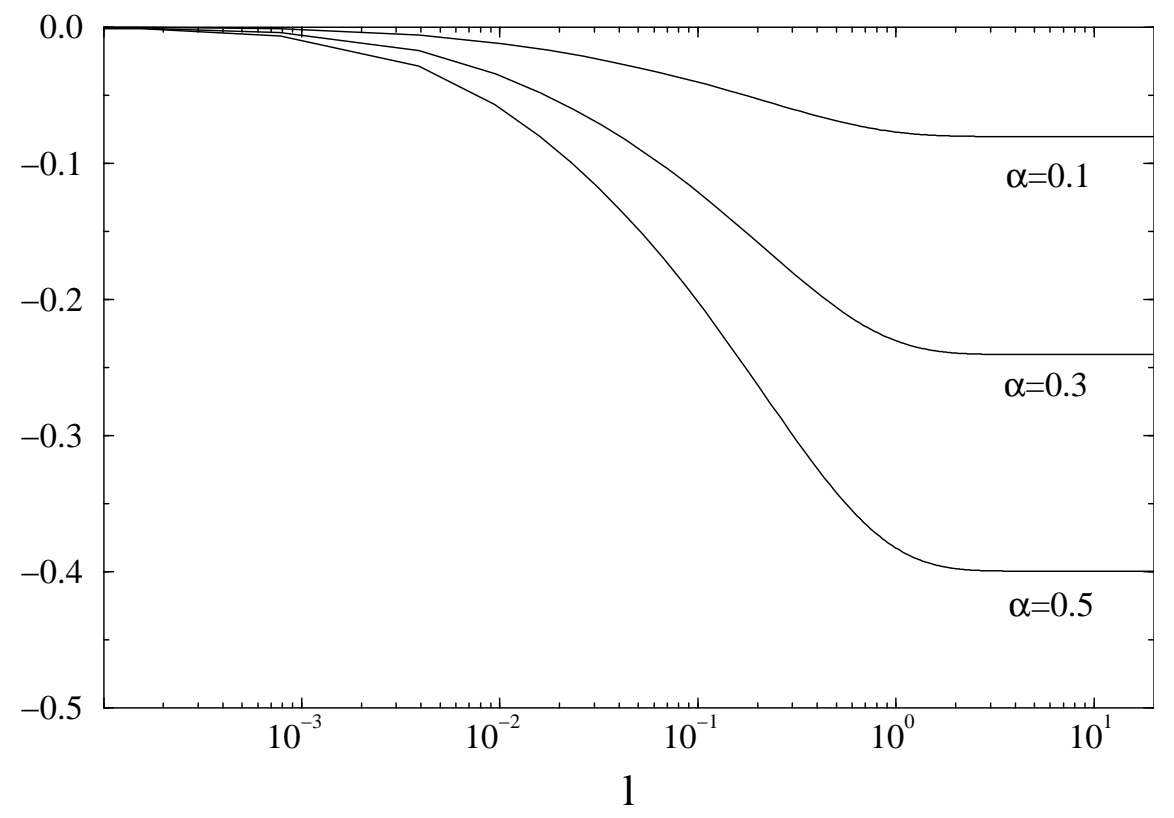

Figure 2.1: Ground-state energy of the polaron in the flow equations as a function of the flow parameter $l$ for $\alpha=0.1,0.3,0.5$ and $D=10$.

of $\Delta_{k, q}$ on the right-hand side, perturbation theory of first order is readily reproduced for $|k|<\sqrt{2}$.

However, here we will solve the set of equations exactly using numerical methods. A description of these methods is given in Appendix E.1. The $q$ integration in Eq. (2.48) is performed using the extended trapezoidal rule. Accurate results are obtained for a discretization $\delta q \approx 0.15$. Fig. 2.1 shows the solution for $\epsilon_{0}(l)$ for different coupling strengths $\alpha$ and the bandwidth $D=10$.

The ground-state energy $E_{0}$ as a function of $\alpha$ for $D=10$ is shown in Fig. 2.2. One recognizes that the result of the flow equations for the ground-state energy is even larger than the result of the perturbation theory of first order. As both the result of perturbation theory of first order and Feynman's result are upper bounds for the ground-state energy, one concludes that this standard approximation for the flow equations gives poor results for the polaron's energy. From Fig. 2.3 we see that this statement also holds for different bandwidths.

Differentiating Eq. (2.48) twice with respect to $k$ gives an equation for the inverse effective mass of the polaron. Fig. 2.4 shows the solution for $d^{2} /\left.d k^{2} \epsilon_{k}(l)\right|_{k=0}$ for different coupling strengths $\alpha$ and the bandwidth $D=10$.

The $\alpha$ dependence of the inverse effective mass $1 / m=\lim _{l \rightarrow \infty} d^{2} /\left.d k^{2} \epsilon_{k}(l)\right|_{k=0}$ for $D=10$ is shown in Fig. 2.5. The result of the flow equations for the effective mass is larger than those of Feynman or Lee, Low and Pines. For couplings larger than $\alpha \approx 2$ this method fails in calculating the mass, similarly to perturbation theory a singularity for the mass is obtained. Varying the bandwidth the result of the flow equations stays close 


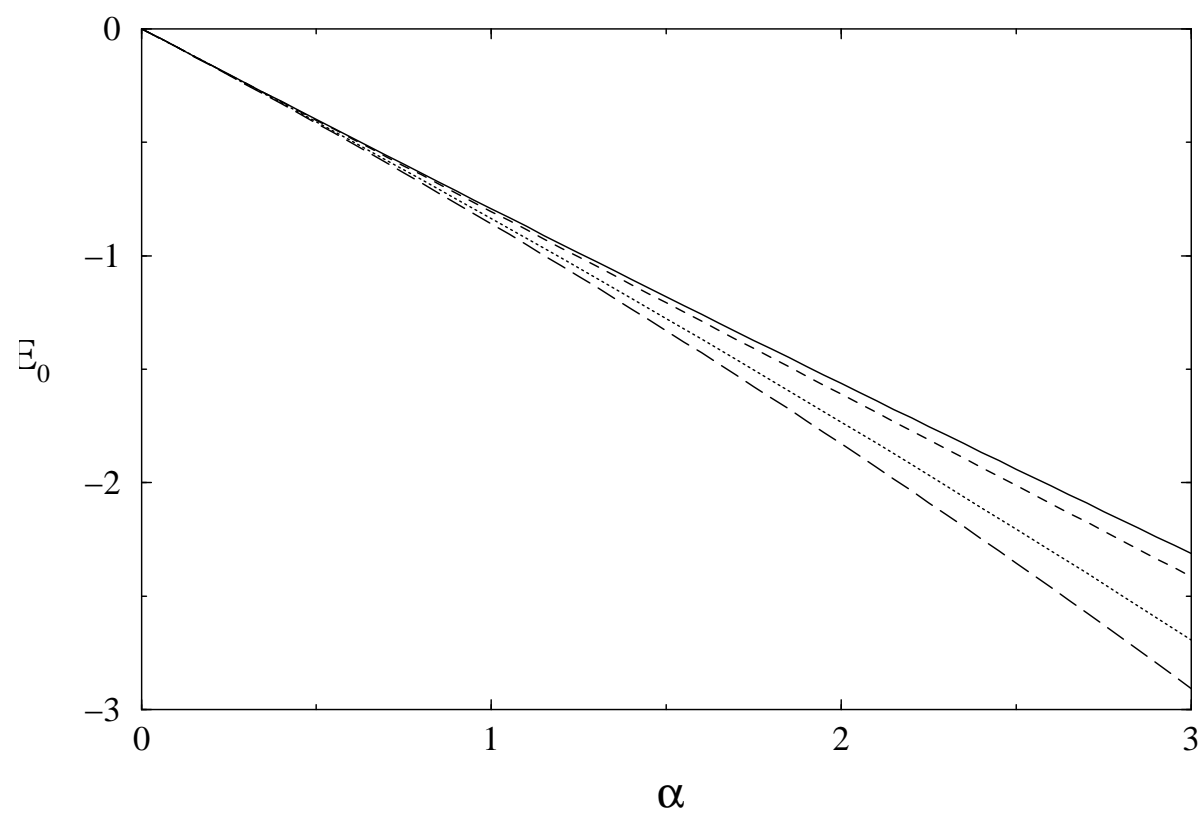

Figure 2.2: Ground-state energy of the polaron as a function of the coupling constant $\alpha$ for $D=10$. Solid line: flow equations. Dashed and long-dashed lines: perturbation theory of order $\alpha$ and $\alpha^{2}$. Dotted line: Feynman's method.

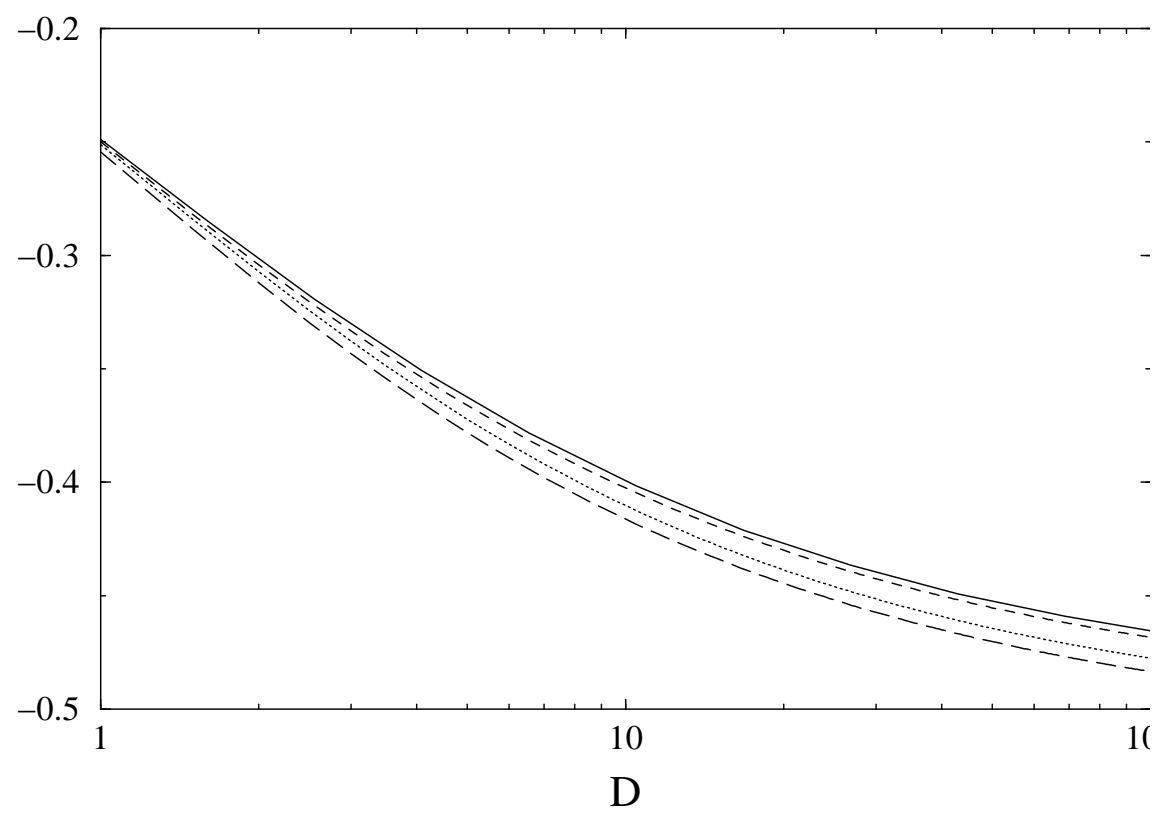

Figure 2.3: Ground-state energy of the polaron as a function of the bandwidth $D$ for $\alpha=0.5$. Solid line: flow equations. Dashed and long-dashed lines: perturbation theory of order $\alpha$ and $\alpha^{2}$. Dotted line: Feynman's method. 


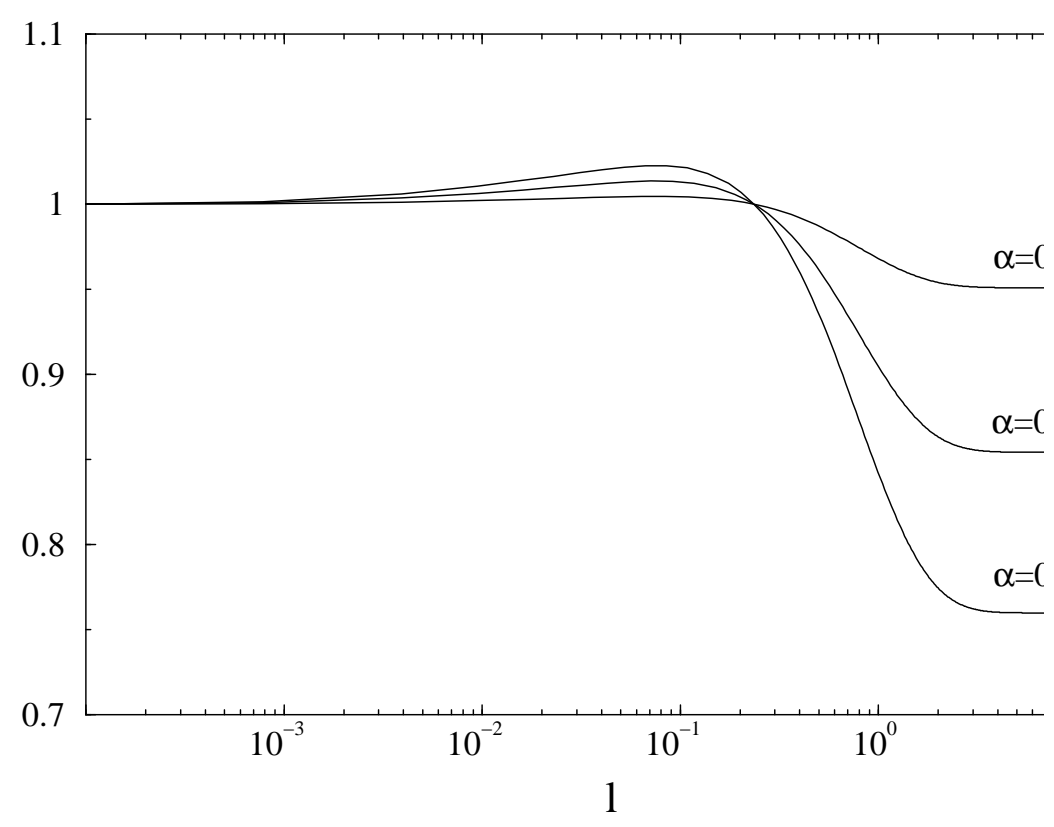

Figure 2.4: Inverse effective mass of the polaron in the flow equations as a function of the flow parameter $l$ for $\alpha=0.1,0.3,0.5$ and $D=10$.

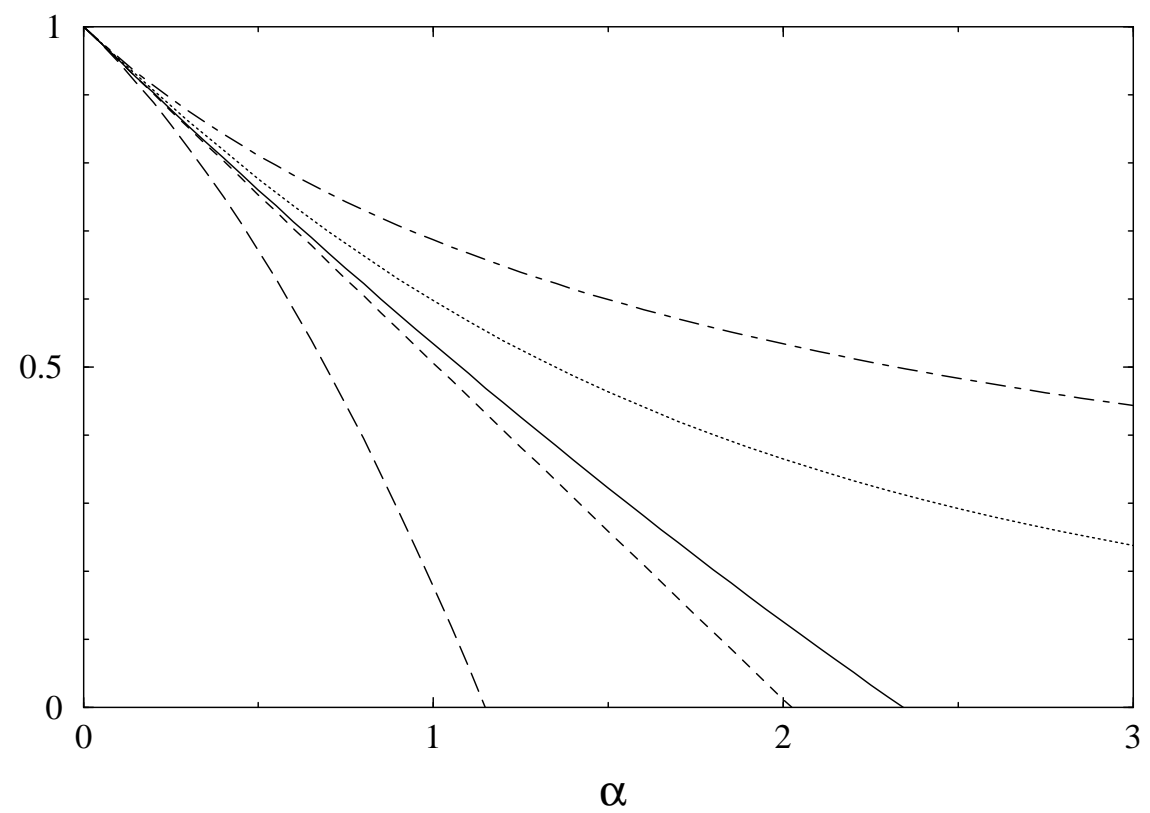

Figure 2.5: Inverse effective mass of the polaron as a function of the coupling constant $\alpha$ for $D=10$. Solid line: flow equations. Dashed and long-dashed lines: perturbation theory of order $\alpha$ and $\alpha^{2}$. Dotted line: Feynman's method. Dot-dashed line: result of Lee, Low and Pines. 


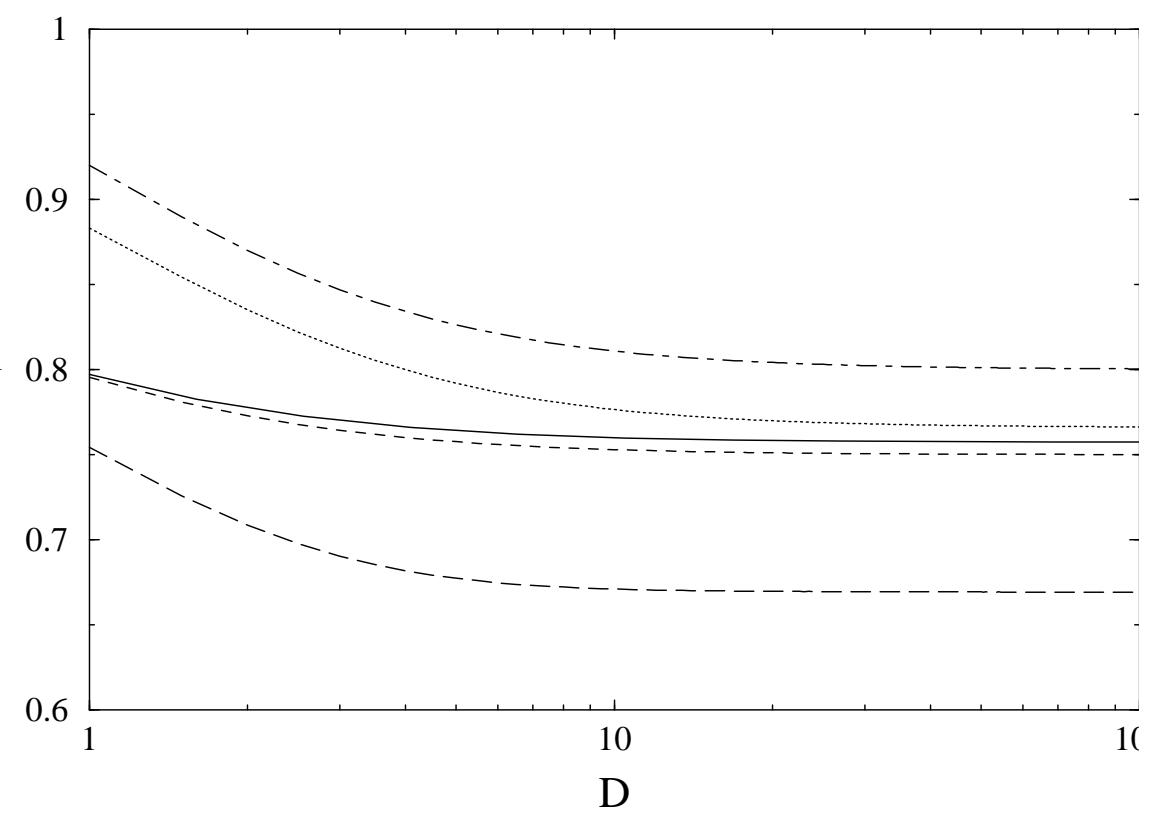

Figure 2.6: Inverse effective mass of the polaron as a function of the bandwidth $D$ for $\alpha=0.5$. Solid line: flow equations. Dashed and long-dashed lines: perturbation theory of order $\alpha$ and $\alpha^{2}$. Dotted line: Feynman's method. Dot-dashed line: result of Lee, Low and Pines.

to that of perturbation theory of first order (see Fig. 2.6).

\subsection{Real-time renormalization-group for the polaron}

Since we deal with vanishing temperature, we may use the Gell-Mann-Low theorem to determine the renormalized energies $E_{k}$. Thus, we assume that the interaction is turned on adiabatically in the interval $t \in]-\infty, 0]$ and that there is no crossing of the ground-states during this process. We therefore consider the $S$ matrix

$$
\begin{aligned}
S & =\lim _{t_{0} \rightarrow \infty} T e^{-i \int_{-t_{0}}^{t} d t H} \\
& =\lim _{t_{0} \rightarrow \infty} e^{-i\left(H_{0}+H_{B}\right) t} T e^{-i \int_{-t_{0}}^{t} d t^{\prime} H_{V}\left(t^{\prime}\right)} e^{-i\left(H_{0}+H_{B}\right) t_{0}}
\end{aligned}
$$

acting on the free ground-state with momentum $k, c_{k}^{\dagger}|0\rangle$. Here, $T$ denotes time ordering and $H_{V}(t)$ is the interaction part of the Hamiltonian taken in the interaction picture with respect to $H_{0}+H_{B}$. The idea of the RTRG is to leave this object invariant while successively integrating out diagrams of different time scales. Thereby a renormalized propagator is generated. Its matrix elements correspond to the renormalized energies according to the Gell-Mann-Low theorem. The general formulation of the RTRG will be explained in detail in Section 3.2. Thus, here we only mention the main points, which this 


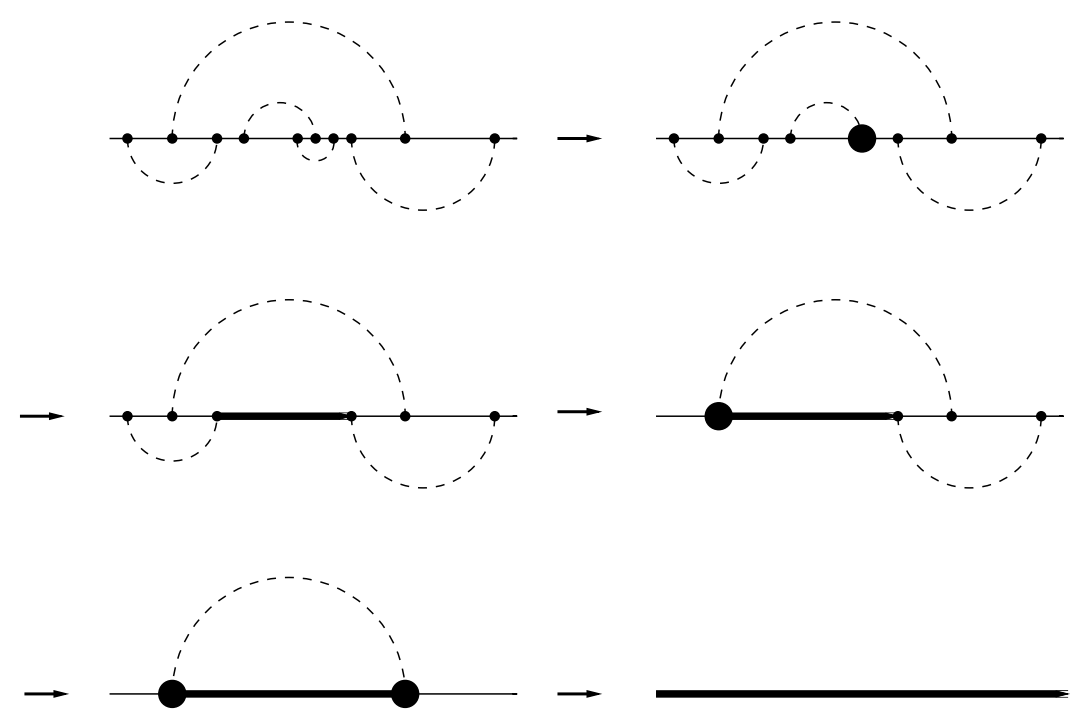

Figure 2.7: Scheme of the real-time renormalization-group. With increasing cutoff $t_{c}$ (indicated by the arrows) the correlation functions (dashed lines) are successively accounted for by renormalized energies and coupling constants (thick lines and dots).

systematic approach is based on. The procedure is schematically shown in Fig. 2.7 [12]. For a given cutoff $t_{c}$ in time space, we allow only for correlation functions of the phonons with a time scale $t>t_{c}$. At zero temperature, the latter are given by

$$
\left\langle\left(a_{-q}^{\dagger}+a_{q}\right)(t)\left(a_{q}^{\dagger}+a_{-q}\right)\right\rangle=e^{-i \omega t} .
$$

All correlation functions (dashed lines in Fig. 2.7) with time scales shorter than $t_{c}$ are accounted for by renormalized energies and coupling constants, which then are $t_{c}$ dependent. These are represented by thick lines and dots in Fig. 2.7. A change of $t_{c}$ to $t_{c}+d t_{c}$ is made by applying three steps [13]: (i) expanding the second exponential in Eq. (2.50) and introducing normal ordering for the phonon operators using Wick's theorem, (ii) integrating over the contractions with a time scale between $t_{c}$ and $t_{c}+d t_{c}$ and (iii) resumming the operators in an exponential form. Consequently, these operators will not be limited to zero-phonon and one-phonon operators any more. But we shall see that a good approximation is achieved for not too large coupling constants, if we neglect double or higher-order vertex operators. Within this method we only need to consider the operators of the electronic system, as the phonon degrees of freedom are integrated out in each $t_{c}$ step. For the polaron problem we write $H_{V}=\sum_{q} G_{q}\left(a_{-q}^{\dagger}+a_{q}\right)$. Thus we start with $t_{c}=0$ involving the operators

$$
\begin{aligned}
H_{0} & =\sum_{k} \epsilon_{k} c_{k}^{\dagger} c_{k}, \\
G_{q} & =\sum_{k} M c_{k+q}^{\dagger} c_{k}
\end{aligned}
$$


as in Eqs. (2.1) and (2.3) and end up with an effective Hamiltonian for $t_{c} \rightarrow \infty$. We obtain the following RG equations

$$
\begin{aligned}
\frac{d H_{0}}{d t_{c}}= & -i e^{-i \omega t_{c}} \sum_{q} e^{i H_{0} t_{c}} G_{q} e^{-i H_{0} t_{c}} G_{-q} \\
\frac{d G_{q}}{d t_{c}}= & -e^{-i \omega t_{c}} \sum_{q^{\prime}} \int_{0}^{t_{c}} d t\left(e^{i H_{0} t} G_{q^{\prime}} e^{-i H_{0} t} G_{q} e^{i H_{0}\left(t-t_{c}\right)} G_{-q^{\prime}} e^{-i H_{0}\left(t-t_{c}\right)}\right. \\
& \left.-G_{q} e^{i H_{0} t} G_{q^{\prime}} e^{-i H_{0} t_{c}} G_{-q^{\prime}} e^{-i H_{0}\left(t-t_{c}\right)}\right)
\end{aligned}
$$

The second term in Eq. (2.55) is a correction term. It is due to the fact that a time interval connected with a contraction becomes a single point in time at one RG step. In the next step this leads to the generation of new terms which were previously not present, and therefore, must be subtracted. Taking the corresponding matrix elements of Eqs. (2.54) and (2.55) we obtain

$$
\begin{aligned}
& \frac{d \epsilon_{k}}{d t_{c}}=-i \sum_{q} e^{-i \Delta_{k, q} t_{c}} M_{k+q,-q} M_{k, q} \\
& \frac{d M_{k, q}}{d t_{c}}=\sum_{q^{\prime}}\left(\begin{array}{l}
M_{k+q+q^{\prime},-q^{\prime}} M_{k+q^{\prime}, q} M_{k, q^{\prime}} \frac{e^{-i \Delta_{k+q, q^{\prime}} t_{c}}-e^{-i \Delta_{k, q^{\prime}} t_{c}}}{i\left(\Delta_{k+q, q^{\prime}}-\Delta_{k, q^{\prime}}\right)} \\
\left.+M_{k, q} M_{k+q^{\prime},-q^{\prime}} M_{k, q^{\prime}} t_{c} e^{-i \Delta_{k, q^{\prime}} t_{c}}\right)
\end{array}\right)
\end{aligned}
$$

with the $t_{c}$ dependent coefficients $\epsilon_{k}\left(t_{c}\right)$ and $M_{k, q}\left(t_{c}\right)$. Again the energy $\Delta_{k, q}$ is defined by Eq. (2.6). The $(k, q)$ dependence of the interaction coefficients is generated during the RG flow. The level broadening is included in Eq. (2.56) since all energies become complex. The terms that generate the double vertex operators are of fourth order in $M$. Therefore Eqs. (2.56) and (2.57) contain the order $M^{4}$ exactly. Thus, the renormalized energies $E_{k}=\lim _{t_{c} \rightarrow \infty} \epsilon_{k}\left(t_{c}\right)$ are correct up to order $\alpha^{2}$. In comparison the method of Lee, Low and Pines, Feynman's approach and the flow equations lead to results which are correct up to first order in $\alpha$ only. If one neglects the $t_{c}$ dependence of $M_{k, q}$ and $\Delta_{k, q}$ and introduces the regularization $e^{-\eta t_{c}}$, Eq. (2.56) reproduces perturbation theory of first order for $|k|<\sqrt{2}$.

To go beyond perturbation theory we solve the ordinary differential equations (2.56) and (2.57) numerically (Appendix E.1). For the integrals a discretization $\delta q \approx 0.15$ is again sufficient. Regarding the oscillating terms in these equations one recognizes that given a certain discretization in $q$ space one obtains large errors with increasing time $t_{c}$. To avoid this $\Delta_{k, q}$ and $M_{k, q}$ have been interpolated in $q$ space (see also Appendix E.3). Unfortunately Eqs. (2.56) and (2.57) do not show a convergent behaviour for the groundstate energy for $t_{c} \rightarrow \infty$ (see Fig. 2.8, where the $t_{c}$ flow of $\epsilon_{0}$ is shown for different $\alpha$ and $D=10$ ). One reason is that there are undamped modes corresponding to high 


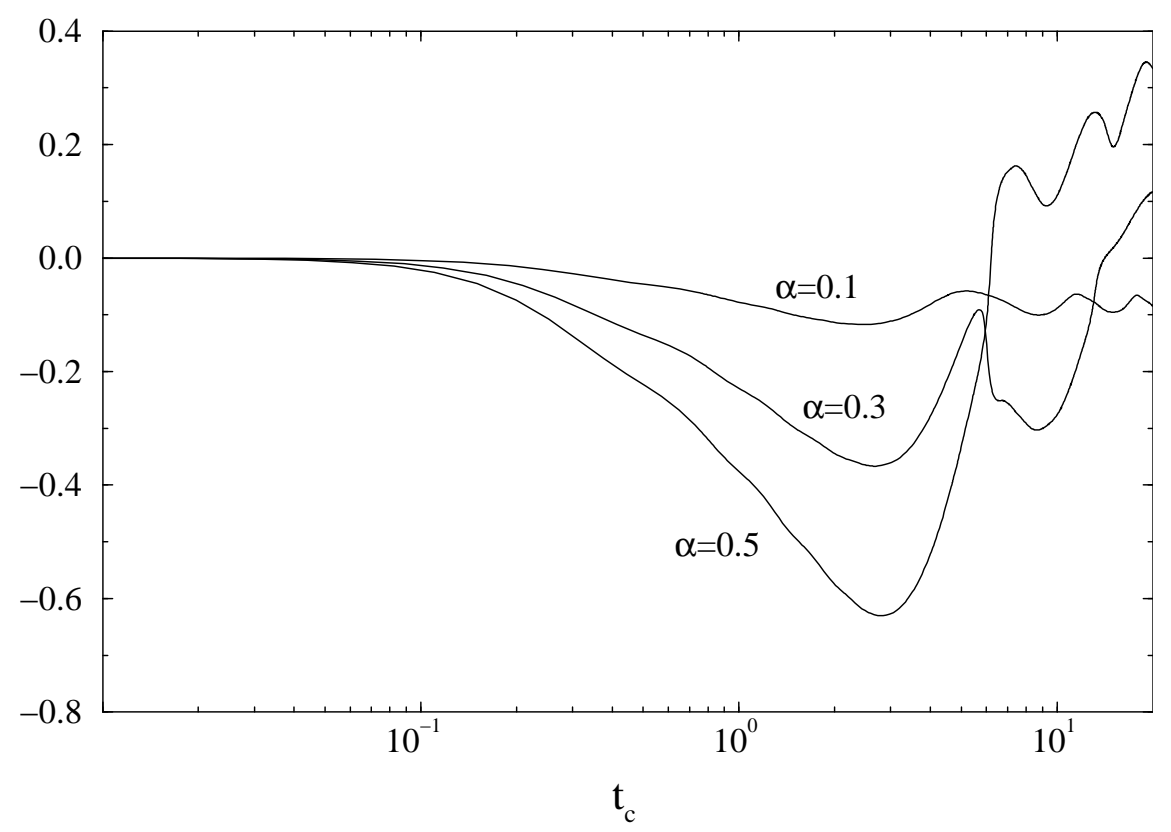

Figure 2.8: Ground-state energy of the polaron in the RTRG as a function of $t_{c}$ for $\alpha=0.1,0.3,0.5$ and $D=10$.

excitations in the $q$ sums leading to increasing effects on the ground-state energy. In this context another problem arises from the fact that the correlation function in Eq. (2.51) is not decaying. As a consequence, oscillations decay as a function of $t_{c}$ but reoccur for sufficiently large $t_{c}$. For other correlation functions, e.g. for acoustic phonons, the numerics is expected to be more stable.

The idea of our solution is to neglect further renormalizaton effects of both $\Delta_{k, q}$ and $M_{k, q}$ for $t_{c}$ larger than a certain point $t_{f}$ [24]. By doing this we obtain a damped oscillation of the ground-state energy $\epsilon_{0}$ in $t_{c}$ space for $t_{c}>t_{f}$. Therefore Eq. (2.56) can be integrated analytically which leads to

$$
E_{0} \approx \epsilon_{0}\left(t_{f}\right)-\sum_{q} \frac{M_{q,-q}\left(t_{f}\right) M_{0, q}\left(t_{f}\right)}{\Delta_{0, q}\left(t_{f}\right)} e^{-i \Delta_{0, q}\left(t_{f}\right) t_{f}} .
$$

In Fig. 2.9 the solution of Eq. (2.58) is shown as a function of $t_{f}$ for different $\alpha$. The problems mentioned above make it necessary to choose a finite $t_{f}$ where the renormalization effects beyond perturbation theory are contained but the numerical instabilities do not yet occur. We choose $t_{f}=2.5$ for all values of $\alpha$. At this point only low excitations $(\Delta<0.4)$ are not integrated out yet. Since $\Delta \sim 1$ sets the scale for the first excited state, it is reasonable to assume that excited states do not have further important renormalization effects. The change of $E_{0}$ between $t_{f}=2$ and $t_{f}=3$ is approximately $1 \%$ for $\alpha=0.5$.

The $\alpha$ dependence of $E_{0}$ is shown in Fig. 2.10. One notices that for $\alpha \lesssim 1$ we obtain lower values for the ground-state energy than those of both Feynman's method and perturbation theory of second order. However, note that our result does not follow from 


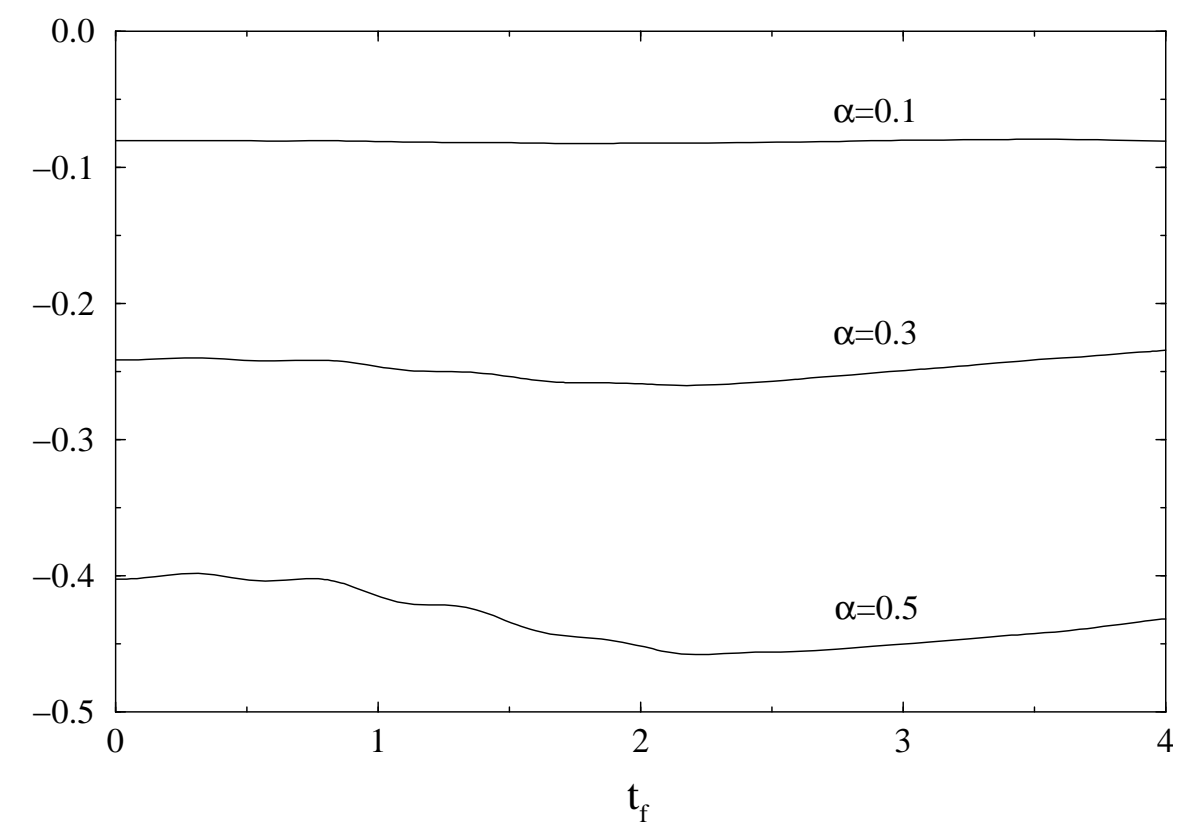

Figure 2.9: Ground-state energy of the polaron in the RTRG as a function of $t_{f}$ for $\alpha=0.1,0.3,0.5$ and $D=10$.

a variational principle and therefore does not give an upper bound for the energy. For larger couplings our method is no longer reliable and yields worse results (see Fig. 2.10), which is not surprising since we neglected double and higher-order vertex operators. We also calculated $E_{0}$ for different bandwidths $D$. As one can see in Fig. 2.11 we find lower ground-state energies for all bandwidths.

To calculate the inverse effective mass one may again (as in Section 2.2) differentiate the flow equation for the energy, Eq. (2.56), twice with respect to $k$. However, the numerical solution of the resulting equation exhibits an even worse convergence than in case of the energy. This behaviour can easily be understood in the perturbative solution of first order, where one again neglects the $t_{c}$ dependence of $M_{k, q}$ and $\Delta_{k, q}$. The differentiation leads to oscillations with increasing amplitude. To reproduce the result of perturbation theory one again has to introduce the regularization $e^{-\eta t_{c}}$.

For the numerical solution of the differential equations, we again introduce a finite $t_{f}$ to avoid these problems. However, the accuracy for the mass is worse than for the energy. For $\alpha=0.5$ the change of $1 / \mathrm{m}$ is approximately $7 \%$ between $t_{f}=2$ and $t_{f}=3$, see Fig. 2.12.

The results as a function of $\alpha$ are shown in Fig. 2.13. For small couplings $(\alpha \lesssim 0.5)$, we find a value for the mass between the variational principle of Feynman and the one of Lee, Low and Pines. For larger couplings the numerical solution is too unstable to make definite statements from the RG approach. From Fig. 2.14 we see that our mass depends only slightly on the bandwidth. 


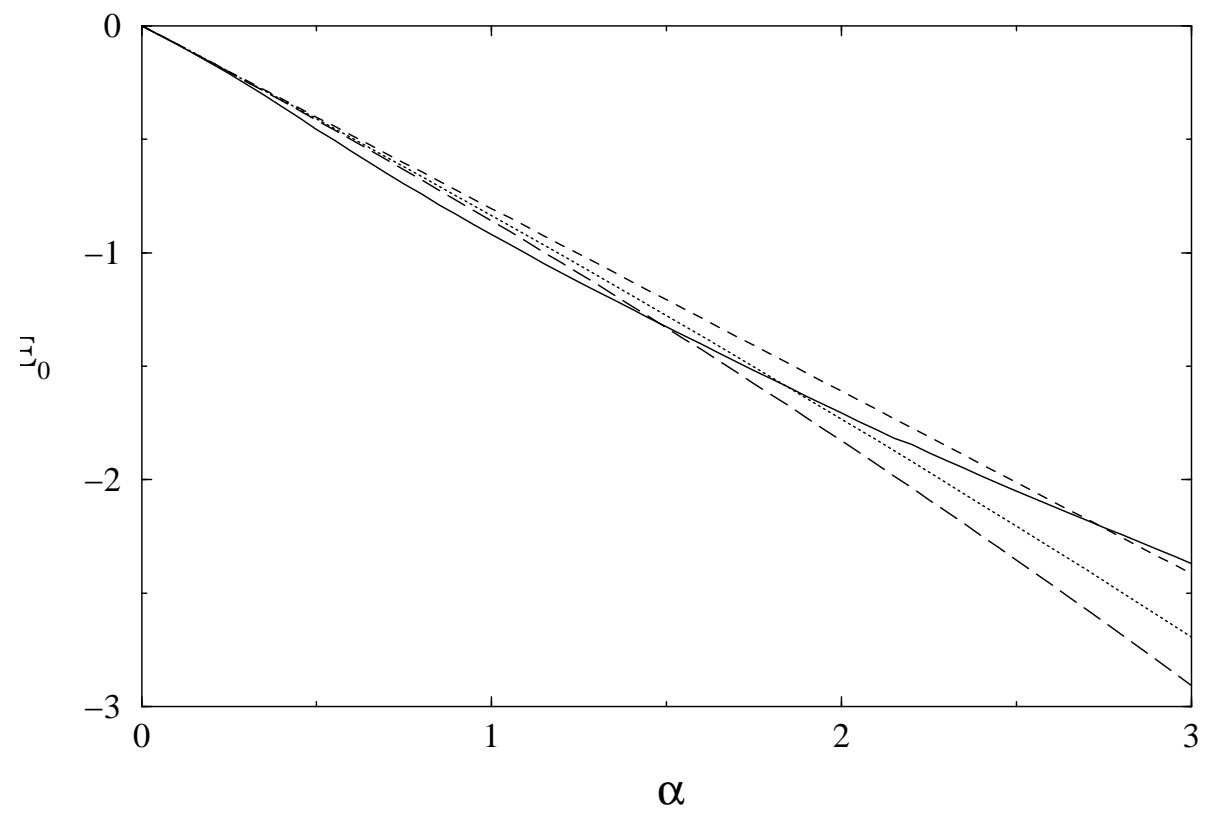

Figure 2.10: Ground-state energy of the polaron as a function of the coupling constant $\alpha$ for $D=10$. Solid line: RTRG with $t_{f}=2.5$. Dashed and long-dashed line: perturbation theory of order $\alpha$ and $\alpha^{2}$. Dotted line: Feynman's method.

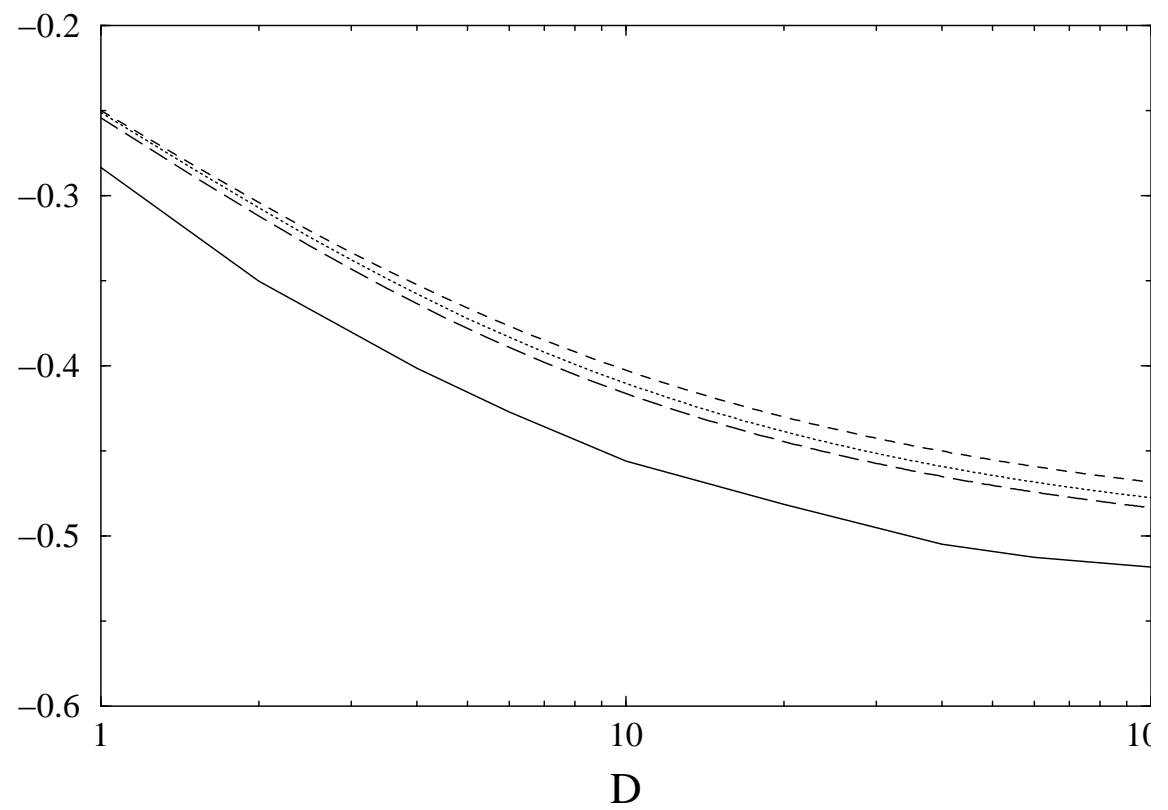

Figure 2.11: Ground-state energy of the polaron as a function of the bandwidth $D$ for $\alpha=0.5$. Solid line: RTRG with $t_{f}=2.5$. Dashed and long-dashed line: perturbation theory of order $\alpha$ and $\alpha^{2}$. Dotted line: Feynman's method. 


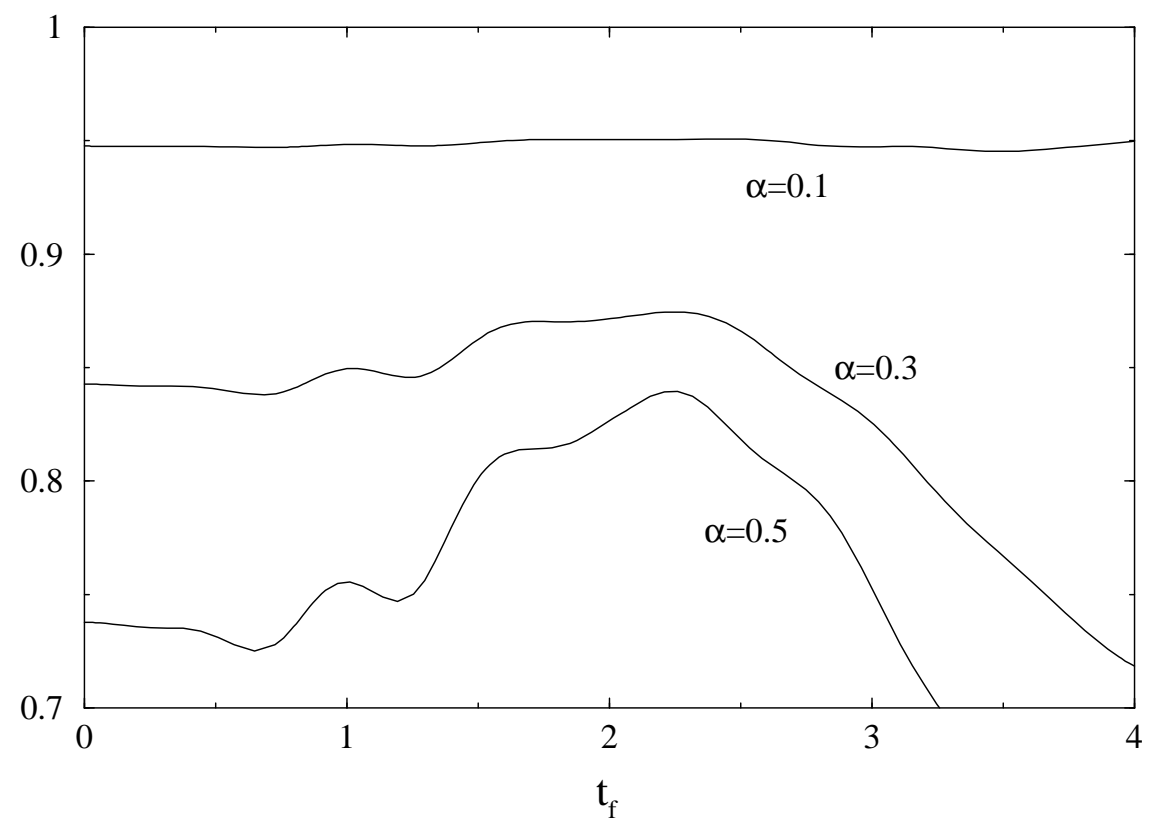

Figure 2.12: Inverse effective mass of the polaron in the RTRG as a function of $t_{f}$ for $\alpha=0.1,0.3,0.5$ and $D=10$.

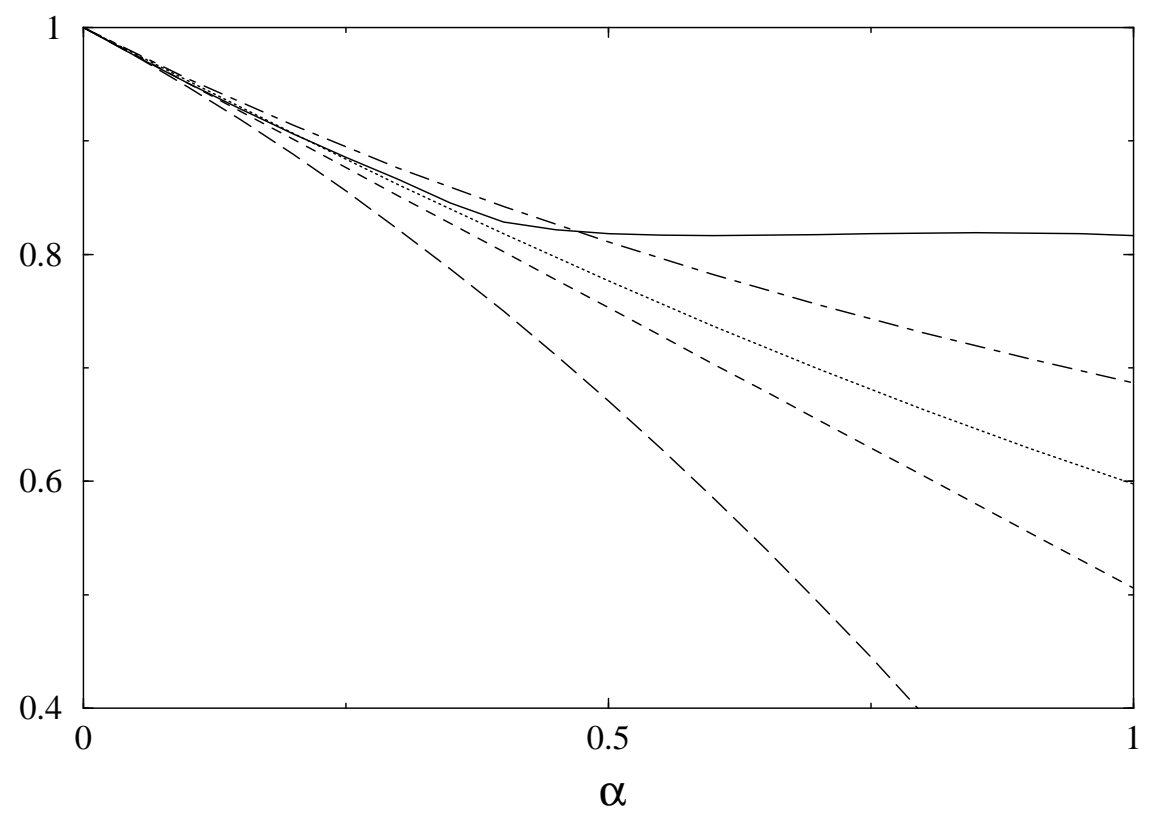

Figure 2.13: Inverse effective mass of the polaron as a function of the coupling constant $\alpha$ for $D=10$. Solid line: RTRG with $t_{f}=2.5$. Dashed and long-dashed line: perturbation theory of order $\alpha$ and $\alpha^{2}$. Dotted line: Feynman's method. Dot-dashed line: result of Lee, Low and Pines. 


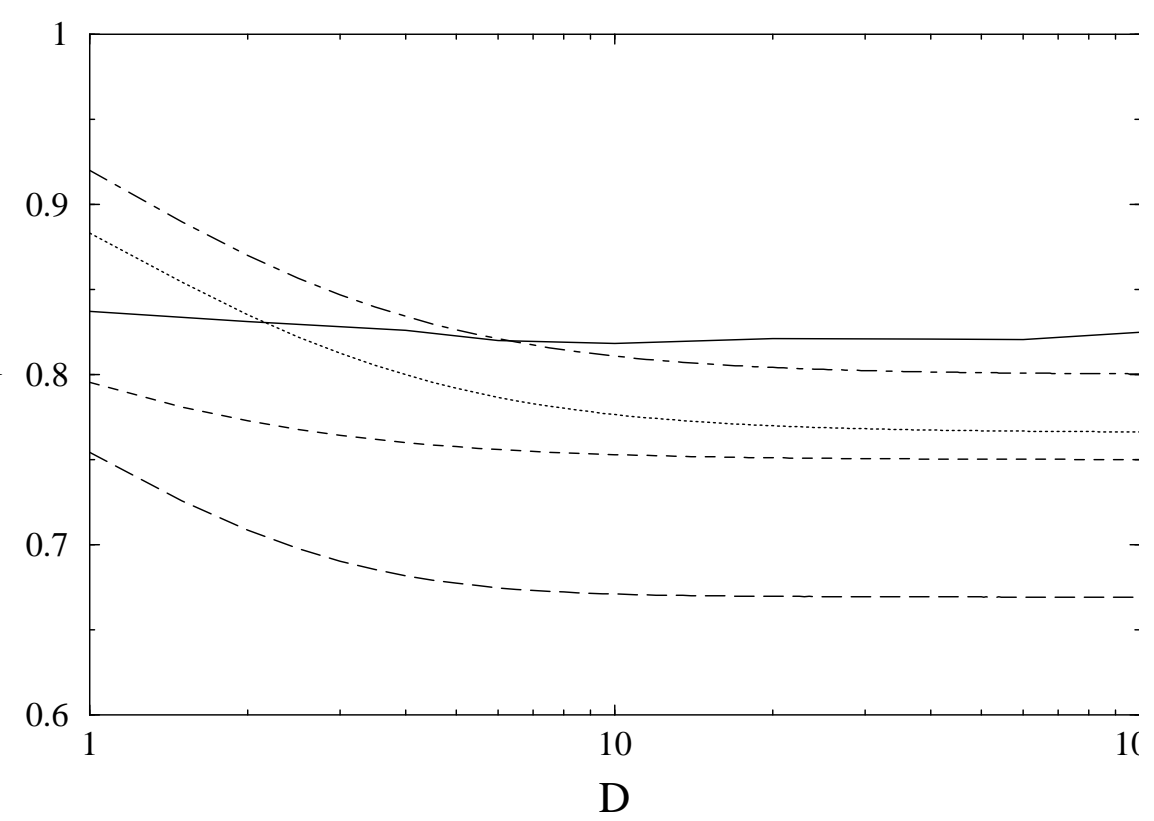

Figure 2.14: Inverse effective mass of the polaron as a function of the bandwidth $D$ for $\alpha=0.5$. Solid line: RTRG with $t_{f}=2.5$. Dashed and long-dashed line: perturbation theory of order $\alpha$ and $\alpha^{2}$. Dotted line: Feynman's method. Dot-dashed line: result of Lee, Low and Pines.

\subsection{Discussion}

In summary, we applied two different renormalization-group methods to the one-dimensional polaron problem. We also generalized the methods of Lee, Low and Pines as well as that of Feynman to the one-dimensional case with finite bandwidth. We compared the values for the ground-state energy and the effective mass, where we also considered the results of perturbation theory. The following conclusions can be drawn:

If in the formalism of the flow equations one uses the approximation introduced in Ref. [49], Eqs. (2.48) and (2.49) are obtained. They are not sufficient to analyze the polaron beyond perturbation theory. In fact, they yield even worse results than simple perturbation theory of first order. To improve the performance of this formalism one may, of course, extend the set of flow equations, i.e. account for double phonon processes [48].

In contrast, the real-time renormalization-group method is able to study the polaron problem beyond perturbation theory. However, due to the fact, that the bath correlation function is not decaying, we did not obtain a convergent solution of the RG equations for $t_{c} \rightarrow \infty$. We applied a physically motivated approximation by introducing the parameter $t_{f}$, which made it possible to obtain results within relatively small errors [24]. For the effective mass the accuracy is generally worse than for the ground-state energy. The RTRG gives reliable results only for not too large couplings: $\alpha \lesssim 1$ concerning the ground-state energy and $\alpha \lesssim 0.5$ regarding the effective mass. The restriction in $\alpha$ is due to the ne- 
glecting of double and higher-order vertex operators. In this context note that it is difficult to judge the performance of the RTRG for the polaron, because there are no exact results which could serve as a test for our approach. In summary, it remains unsatisfactory that the reliability of the RTRG method for the polaron model suffers from the convergence problems. Additionally, due to the continuous electron spectrum a numerical calculation of the reduced density matrix is not viable.

We will show in the next chapter that this method works much better with damped bath correlations. Furthermore, in the next chapter we will study a problem with only two degrees of freedom in the local system. Thus the computational effort needed will be much less in this case, so that we will be able to use this approach to calculate the time-dependent reduced density matrix starting from an arbitrary noneqilibrium state. 



\section{Chapter 3}

\section{The spin-boson model}

The spin-boson model (SBM) is one of the most fundamental quantum dissipative systems [27] (for a review, see Ref. [1]). It models a particle in a double-well potential with a finite tunnel amplitude coupled to a (bosonic) heat bath of harmonic oscillators, see Fig. 3.1. For various physical and chemical systems this rather simple model is adequate and captures the essential physics. For instance the model can be applied to electron transfer reactions, where an electron tunnels from a donor site to an acceptor site in a polarized environment interacting with the electron [53]. Another example is quantum tunneling between flux states in a SQUID [54]. Other applications include tunneling of light particles or defects in solids [55,56] and electron tunneling between quantum dots [30]. An example of the latter and its relation to the spin-boson model will be studied in Chapter 4 . The strong interest in the spin-boson model is due to the fact that it provides a nontrivial description of dissipation in these quantum systems.

We study the dynamics of the spin-boson model using the real-time renormalizationgroup method. In Chapter 2 we used this method to study an equilibrium problem for vanishing temperature, see also Refs. $[13,24]$. Now we apply its nonequilibrium formulation for arbitrary temperature $[12,14]$ to the spin-boson model. For the first time we use this approach to calculate the time-dependent reduced density matrix, and, furthermore generalize the formalism to the calculation of equilibrium correlation functions [25, 26].

We present a solution of the complete dynamics of the reduced density matrix of the spin-boson model, starting from an arbitrary nonequilibrium state. This is achieved for arbitrary parameters with the only restriction of not too large coupling strengths. We also determine the oscillation frequency of the diagonal elements, and in the asymptotic regime we find an exponential decay of the elements of the reduced density matrix involving decay constants, which are identified with a dephasing time and a relaxation time. Additionally, we calculate the spin susceptibility as well as equilibrium correlation functions. Comparisons to the noninteracting blip approximation (NIBA) [27] and to results for the anisotropic Kondo model $[28,29]$ are made. In contrast to the NIBA our analysis provides the complete dynamics of the spin-boson model, i.e. all elements of the time-dependent reduced density matrix are determined. Furthermore, we obtain accurate results in a parameter regime, where the NIBA fails. Generally, our calculation 


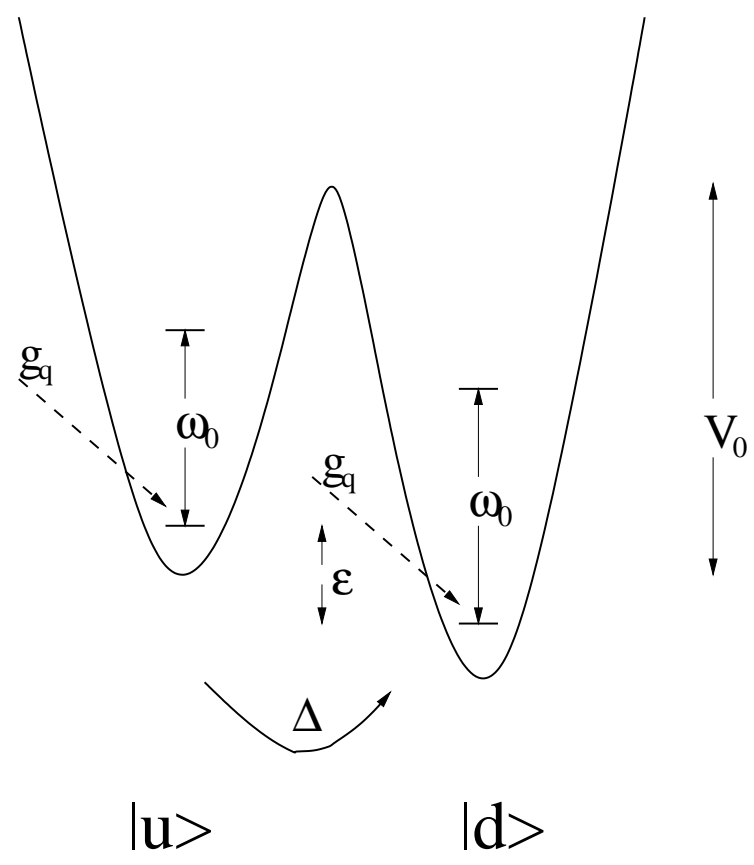

Figure 3.1: Double-well potential for a particle coupled to a bosonic bath (dashed lines). $g_{q}$ : coupling strength to the coordinate of the $q$ th oscillator of the bath, $\Delta$ : tunnel matrix element, $\epsilon$ : bias, $\omega_{0}$ : lowest excitation energies, $V_{0}$ : potential barrier. Truncating the problem to the ground-states $|u\rangle$ and $|d\rangle$ leads to the spin-boson model.

allows a comparison to the anisotropic Kondo model in a parameter regime, which is not accessible by many other methods. To demonstrate the reliability of our results we show the consistency with chromostochastic quantum dynamics (CSQD) [57], and check the Shiba-relation as well as the scaling behaviour [25, 26].

\subsection{Model Hamiltonian}

The general Hamiltonian of a local system with the generalized coordinate $z$, which is coupled to an environment consisting of harmonic oscillators with coordinates $x_{q}$, can be written as

$$
H=\frac{p_{z}^{2}}{2 M}+V(z)+\frac{1}{2} \sum_{q}\left(\frac{p_{q}^{2}}{m_{q}}+m_{q} \omega_{q}^{2}\left(x_{q}-\frac{c_{q}}{m_{q} \omega_{q}^{2}} z\right)^{2}\right) .
$$

Here, the local system is characterized by the mass $M$ and the general potential $V(z)$. Furthermore, we introduced $m_{q}$ and $\omega_{q}$ as the masses and frequencies of the oscillators. Finally, we assumed an interaction which is linear in both $x_{q}$ and $z$ involving the coupling constants $c_{q}$. This model is known as Caldeira-Leggett model [58].

Let us now specialize to the spin-boson model, where the local system is given by a particle in a double-well potential (Fig. 3.1). According to Eq. (3.1) we write the spin- 
boson Hamiltonian as the sum $H=H_{0}+H_{B}+H_{V}$, where $H_{0}$ is the Hamiltonian of the particle in the double-well potential, $H_{B}$ the Hamiltonian of the oscillators in the bath and $H_{V}$ the contribution of the interaction between those systems. In the following we set $\hbar=k_{B}=1$.

The two ground-states for the particle in the double-well potential are denoted by $|u\rangle$ and $|d\rangle$. Apart from the tunnel matrix element $\Delta$, which follows from a WentzelKramers-Brillouin calculation, there may also be a finite energy difference $\epsilon$. If for the potential barrier $V_{0}$, the lowest excitation energies $\omega_{0}$ and the bath temperature $T$ the relation $V_{0} \gg \omega_{0} \gg \Delta, \epsilon, T$ holds, we may use a tight-binding model to describe the problem. Thereby the local system can be represented by only two states: $|u\rangle$ and $|d\rangle$. Using pseudospin language we then obtain the Hamiltonian for the two-state system as

$$
H_{0}=-\frac{\Delta}{2} \sigma_{x}+\frac{\epsilon}{2} \sigma_{z}
$$

where $\sigma_{x}$ and $\sigma_{z}$ are the usual Pauli matrices.

The bath part of the Hamiltonian is the same as in the Caldeira-Leggett model (see Eq. (3.1)).

$$
H_{B}=\frac{1}{2} \sum_{q}\left(\frac{p_{q}^{2}}{m_{q}}+m_{q} \omega_{q}^{2} x_{q}^{2}\right) .
$$

For the interaction part we write

$$
H_{V}=\sigma_{z} \sum_{q} \sqrt{\frac{m_{q} \omega_{q}}{2}} g_{q} x_{q} .
$$

The term which is proportional to $z^{2}$ in Eq. (3.1) is now absorbed in the local potential $V(z)$, as we assumed Gaussian statistics for the fluctuating force $\sum_{q} c_{q} x_{q}(t)[58,1]$. Furthermore, we introduced a new coupling constant $g_{q}$, which contains the dependence of $H_{V}$ on the spatial distance between the two wells of the potential. Eventually, quantizing the coordinates $x_{q}$ and the momenta $p_{q}$ yields

$$
\begin{array}{r}
H_{B}=\sum_{q} \omega_{q} a_{q}^{\dagger} a_{q}, \\
H_{V}=\frac{\sigma_{z}}{2} \sum_{q} g_{q}\left(a_{q}^{\dagger}+a_{q}\right),
\end{array}
$$

where $a_{q}^{\dagger}\left(a_{q}\right)$ creates (annihilates) a boson with energy $\omega_{q}$. The coupling to the environment is completely defined by the spectral density

$$
J(\omega)=\pi \sum_{q} g_{q}^{2} \delta\left(\omega-\omega_{q}\right)
$$

which is usually parametrized by

$$
J(\omega)=2 \pi \alpha \omega^{n+1} e^{-\omega / D} .
$$




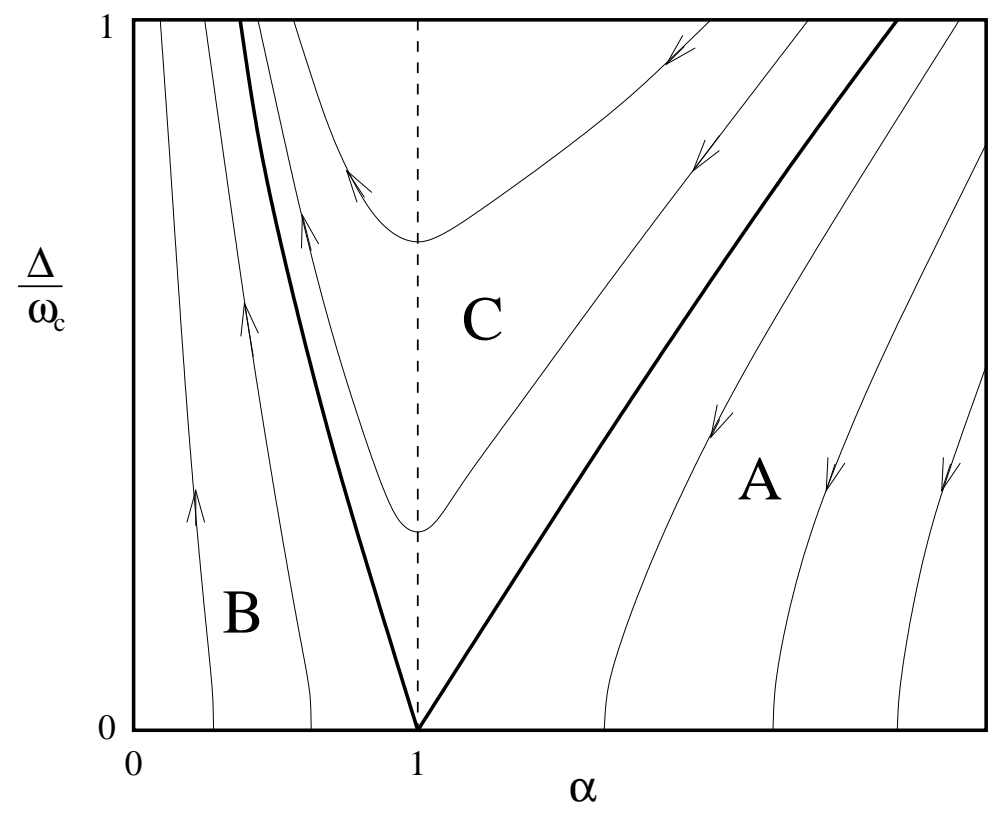

Figure 3.2: Renormalization-group flow of the parameters of the spin-boson model: $\Delta / \omega_{c}$ and $\alpha$. The arrows indicate the direction of decreasing $\omega_{c}$.

The case $n=0$ is referred to as the Ohmic case, whereas $n>(<) 1$ corresponds to the super(sub)ohmic case. In the following we want to consider the Ohmic bath, which most studies of the spin-boson model deal with. Therefore the coupling to the bath is now characterized by the dimensionless coupling constant $\alpha$ and the high-energy cutoff $D$.

An exact solution of the spin-boson model is only known for $\alpha=1 / 2$ [59] which is referred to as the Toulouse limit. From the Poor Man's scaling equations of the anisotropic Kondo model (see Appendix B) one obtains for the running couplings of the spin-boson model $[28,4]$

$$
\begin{aligned}
\frac{d \alpha}{d \ln \omega_{c}} & =\alpha\left(\frac{\Delta}{\omega_{c}}\right)^{2}+\mathcal{O}\left(\frac{\Delta}{\omega_{c}}\right)^{4}, \\
\frac{d\left(\Delta / \omega_{c}\right)}{d \ln \omega_{c}} & =-(1-\alpha)\left(\frac{\Delta}{\omega_{c}}\right)+\mathcal{O}\left(\frac{\Delta}{\omega_{c}}\right)^{3} .
\end{aligned}
$$

The initial conditions are fixed at $\omega_{c}=D$, then $\alpha$ and $\Delta$ are the parameters of the bare Hamiltonian. For decreasing high-energy cutoff $\omega_{c}$ the renormalization-group flow is shown in Fig. 3.2. In the region $A$ - where the coupling constant $\alpha$ is always larger than 1 - we obtain $\Delta / \omega_{c} \rightarrow 0$ for $T=0$. Thus the corresponding parameters give rise to localization. The tunneling regime is found in the regions $B$ and $C$. There the flow of the parameters yields $\alpha \rightarrow 0$ and $\Delta / \omega_{c} \rightarrow \infty$, which can be shown by the numerical renormalization-group or the Bethe ansatz. At $T=0$ the crossover energy scale is defined by $\Delta_{r}=\Delta\left(\tilde{\omega}_{c}\right)$ with $\tilde{\omega}_{c}=\Delta\left(\tilde{\omega}_{c}\right)$. In region $B$ - where the coupling constant $\alpha$ is always less than 1 - one can approximately integrate Eq. (3.10) [28]. This yields

$$
\Delta_{r} / \Delta=(\Delta / D)^{\alpha /(1-\alpha)}
$$


where $\Delta$ is the tunnel matrix element of the bare Hamiltonian. The renormalized tunnel matrix element $\Delta_{r}$ is the relevant energy scale of the problem. In the scaling limit, which is defined by

$$
D \rightarrow \infty, \quad \Delta_{r}=\text { const }
$$

physical quantities only depend on $\Delta_{r}$, i.e. there is no other $D$ dependence. This behaviour is referred to as universal [1]. For $T=\epsilon=0$ there is a transition from coherent oscillations to a pure incoherent decay of $\left\langle\sigma_{z}(t)\right\rangle$ at $\alpha=1 / 2$ [27]. Concerning equilibrium correlation functions the long-time behaviour respectively low-frequency limit is analytically known for vanishing temperature from the Shiba-relation [60] (see Subsection 3.4.4).

Among the approximate solutions of the spin-boson model perturbative approaches in $\alpha$ [61] have the important disadvantage that they disregard the renormalization of the tunnel matrix element. Most of the studies on the problem are based on the influence functional method of Feynman and Vernon [62], like e.g. the NIBA [27], which is explained in Appendix A. The NIBA contains the correct energy scale $\Delta_{r}$ of the problem. However, it does not give the correct long-time behaviour [63]. Furthermore, at low temperature it applies only to the diagonal matrix elements of the reduced density matrix $p(t)$ and breaks down for the biased case $\epsilon \neq 0$. Recent real-time quantum Monte Carlo (QMC) simulations $[64,57,65]$ provide also reliable information on the nondiagonal elements of $p(t)$. But as these methods suffer from the dynamical sign problem, they are quite timeconsuming and have not been able to check the correct long-time behaviour of the correlation functions yet. The flow equation method of Wegner (see Chapter 2) has reproduced the Shiba-relation for the unbiased case and coupling strengths up to $\alpha \sim 0.025-0.05$ (with an error of $3-10 \%$ ) [66], but there only spectral properties of the system have been addressed. Other approaches try to use a mapping of the spin-boson model on the anisotropic Kondo model [67, 68, 69] (see Appendix B), and solve the latter exactly using the numerical renormalization-group (NRG) [6, 70], the Bethe ansatz [28], or the conformal field theory (CFT) [29]. However, the NRG and the Bethe ansatz provide only spectral properties or dynamics at very short time scales [71], and CFT yields a solution only for the diagonal elements of $p(t)$ in case of $\epsilon=0$. Furthermore, and most importantly, the mapping on the Kondo model cannot be proven rigorously, and the relation of the parameters is not precisely known [27, 72]. It is known that the mapping is incorrect for finite high-energy cutoff $D$, but it is at least established that the scaling behaviour in the limit of large $D$ agrees with that of the spin-boson model $[66,6,70,29]$.

\subsection{Real-time renormalization-group formalism}

We use the RTRG method to investigate the spin-boson model. Not only the correct renormalized tunnel matrix element $\Delta_{r}$ is contained in this approach, but we can also calculate the dynamics of the two-state system for an arbitrary initial state and consider arbitrary parameters for not too large couplings $\alpha$. In the following analysis the quantities of interest are the time-dependent reduced density matrix, the static susceptibility 
and equilibrium correlation functions. In contrast to Chapter 2, here we need the full nonequilibrium formulation of the method [12], which is explained in the following. The derivation of the formalism is rather general and can readily be transferred to any other quantum dissipative system, where the coupling is linear in the bath field operators.

In order to calculate the reduced density matrix $p(t)$ one performs two main steps. First one expresses $p(t)$ using a kinetic equation, which involves an integral kernel $\Sigma$ accounting for dissipation. This reduces the problem to that of calculating $\Sigma$. In the second step we explain the RTRG method, which provides a set of differential equations determining $\Sigma$.

In case of equilibrium correlation functions, initial correlations give rise to another integral kernel $\Sigma_{D}$, for which we derive additional $\mathrm{RG}$ equations.

\subsubsection{Kinetic equation}

The dynamics of the density matrix $\rho(t)$ is given by the von Neumann equation

$$
\dot{\rho}=-i[H, \rho] \text {. }
$$

For the time evolution of the reduced density matrix $p(t)$ of the local system this leads to

$$
p(t)=\operatorname{Tr}_{B}\left[e^{-i H t} p(0) \rho_{B}^{e q} e^{i H t}\right],
$$

where $\operatorname{Tr}_{B}$ denotes the trace over the bath degrees of freedom. $p(0)$ is the initial density matrix of the two-state system and $\rho_{B}^{e q}=\exp \left(-H_{B} / T\right)$ the equilibrium density matrix of the bath. We assumed here, that initially, the two-state system is decoupled, and the bath is in thermal equilibrium, $\rho(0)=p(0) \rho_{B}^{e q}$. At $t=0$, the coupling is turned on instantaneously, and the reduced density matrix of the two-state system will evolve into a stationary state. This preparation corresponds to a rarely encountered physical situation. It may be realized, however, in electron transfer reactions where photoinjection suddenly gives rise to a particular electronic donor state. One may also think of a preparation which is physically more relevant, e.g. where one applies a strong bias $\epsilon \gg 1$ for $t<0$ to localize the particle in the right well. In this case the particle would be in equilibrium with the environment. These initial correlations cannot be represented by a factorized form of $\rho(0)$ any more. However, it turns out that for the Ohmic bath the initial correlations are negligible for $D \gg \Delta[1]$.

As in Refs. $[12,14]$ we expand the forward/backward propagators $\exp (\mp i H t)$ in the interaction $H_{V}$, and perform the trace $\operatorname{Tr}_{B}$ by applying Wick's theorem with respect to the bath field operators. All terms can be represented diagrammatically as shown in Fig. 3.3. The vertex operators $-i \sigma_{z} / 2$ are ordered along a closed Keldysh contour. They are connected in pairs by the contractions (dashed lines in Fig. 3.3)

$$
\gamma(t)=\operatorname{Tr}_{B}\left[j(t) j \rho_{B}^{e q}\right]
$$

where we used the interaction picture with respect to $H_{B}$ for the operator $j$

$$
j=\sum_{q} g_{q}\left(a_{q}^{\dagger}+a_{q}\right) .
$$




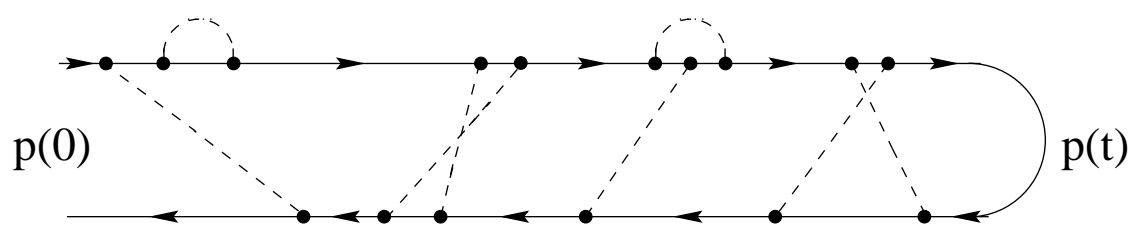

(a) (b) $\quad$ (c) $\quad$ (d)

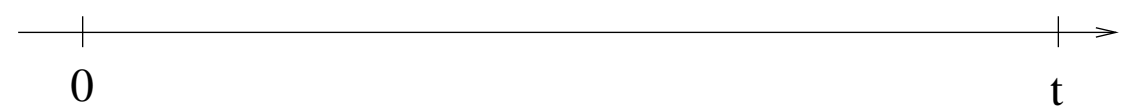

Figure 3.3: Example of a diagram on the Keldysh contour for the reduced density matrix $p(t)$. The dashed lines represent the bath contractions.

By definition

$$
\gamma(-t)=\gamma^{*}(t)
$$

holds. From Eqs. (3.7) and (3.8) one obtains for $\gamma(t)=R(t)+i S(t)$ :

$$
\begin{aligned}
R(t) & =-2 \alpha T \frac{d}{d t} \operatorname{Im}\left[\frac{1}{T / D+i t T}-2 \psi(1+T / D+i t T)\right] \\
S(t) & =-2 \alpha \operatorname{Im}\left[\frac{1}{(t-i / D)^{2}}\right] .
\end{aligned}
$$

Here, we used the logarithmic derivative of the $\Gamma$ function [73]

$$
\psi(x)=\frac{d}{d x} \ln \Gamma(x) .
$$

For the physically relevant situation $D \gg T$ this yields for the real part of $\gamma(t)$

$$
R(t)=-2 \alpha \operatorname{Re}\left[(\pi T)^{2} \frac{1}{\sinh ^{2}(\pi T(t-i / D))}\right] .
$$

The solid line in Fig. 3.3 represents free time evolution of the two-state system. As a result, we have obtained an effective theory of the local system, while the bath has been integrated out.

The type of contractions, which connect vertices lying only on one, the forward or the backward, propagator, has also been present in case of the equilibrium considerations regarding the polaron. In contrast, the contraction lines connecting the forward with the backward propagator do not occur in any equilibrium theory but arise for a Keldysh contour in a natural way. Such contractions lead to non-Hamiltonian dynamics for the reduced density matrix, and therefore, account for dissipation. Formally these two kinds of contractions do not have to be distinguished, when one views the forward and backward propagator as one double line, see Fig. 3.4. The price is that a "state" on the double line 


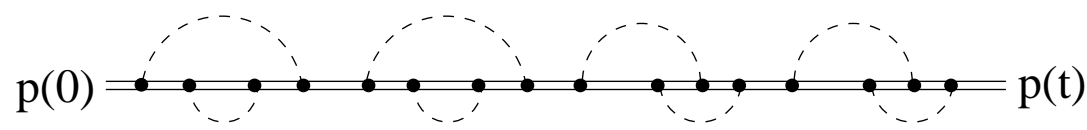

(a)

(b)

(c)

(d)

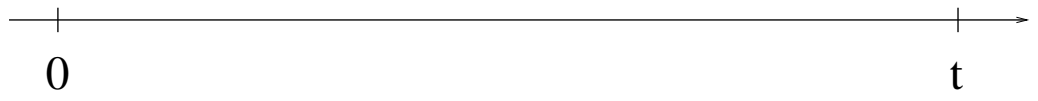

Figure 3.4: The same as Fig. 3.3 but the two lines taken together.

has to be specified by two states, one for the upper (forward) and one for the lower (backward) propagator. Formally this leads to a superoperator notation, where one introduces the Liouvillian $L=L_{0}+L_{B}+L_{V}$, which acts on an usual operator $O$ by taking the commutator $[\cdot, \cdot]_{-}$with the Hamiltonian:

$$
L O=[H, O]_{-}\left(L_{0} O=\left[H_{0}, O\right]_{-}, \quad L_{B} O=\left[H_{B}, O\right]_{-}, \quad L_{V} O=\left[H_{V}, O\right]_{-}\right) .
$$

In Eq. (3.14) the Liouvillian acts on the density matrix $\rho$ :

$$
\begin{aligned}
p(t) & =\operatorname{Tr}_{B}\left[e^{-i L t} p(0) \rho_{B}^{e q}\right] \\
& =\operatorname{Tr}_{B}\left[e^{-i\left(L_{0}+L_{B}\right) t} T e^{-i \int_{0}^{t} d t^{\prime} L_{V}\left(t^{\prime}\right)} p(0) \rho_{B}^{e q}\right] \\
& =\operatorname{Tr}_{B}\left[e^{-i L_{0} t} T e^{-i \int_{0}^{t} d t^{\prime} L_{V}\left(t^{\prime}\right)} p(0) \rho_{B}^{e q}\right]
\end{aligned}
$$

where $T$ denotes the time ordering operator. For $L_{V}(t)$ we used the interaction picture with respect to $L_{0}+L_{B}$. Expanding Eq. (3.22) in $L_{V}$ and performing the trace using Wick's theorem again leads to the effective theory for the local system shown in Fig. 3.4. The free propagation between the vertices is given by the propagator $\exp \left(-i L_{0} t\right)$. For the spin-boson model $L_{0}$ corresponds to a $(4 \times 4)$-matrix, and the elements $\left(L_{0}\right)_{s_{1} s_{1}^{\prime}, s_{2} s_{2}^{\prime}}$ are labeled by the two states of the local system with $s_{1 / 2}\left(s_{1 / 2}^{\prime}\right)$ referring to the forward (backward) propagator. The interaction part $L_{V}$ can be written as

$$
L_{V}=\sum_{p= \pm} G^{p} J^{p} .
$$

The superoperators $G^{p}$ and $J^{p}$ are defined by

$$
\begin{aligned}
G^{+} O & =\frac{\sigma_{z}}{2} O, \\
G^{-} O & =-O \frac{\sigma_{z}}{2}, \\
J^{+} O & =j O, \\
J^{-} O & =O j,
\end{aligned}
$$




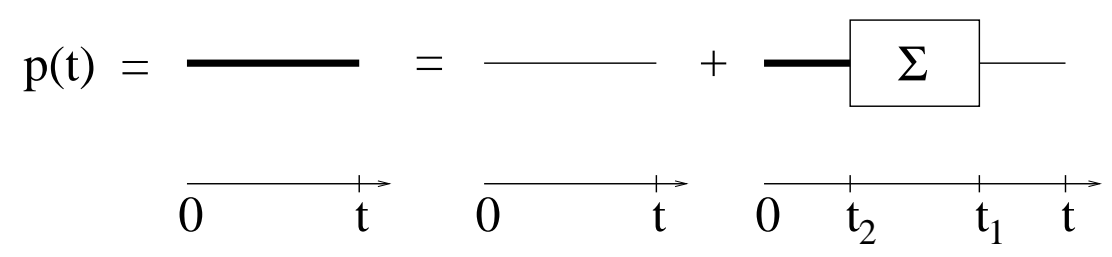

Figure 3.5: Iterative scheme for the reduced density matrix $p(t)$. The two lines of the Keldysh contour are put together to only one line. The thick line represents the exact time evolution of $p(t)$, whereas the thin line corresponds to free propagation. $\Sigma$ represents all irreducible diagrams with the outermost vertices at $t_{2}$ and $t_{1}$.

where $O$ again is an usual arbitrary operator. Thus, in Fig. 3.4 we deal with the interaction vertices $-i G^{p}$, where $p= \pm$ denotes whether the vertex acts on the forward/backward propagator. The contractions connecting the vertices $G^{p_{1}}$ and $G^{p_{2}}$ also depend on $p_{1}, p_{2}$ :

$$
\gamma^{p_{1}, p_{2}}(t)=\operatorname{Tr}_{B}\left[J^{p_{1}}(t) J^{p_{2}} \rho_{B}^{e q}\right] .
$$

This can be expressed using $\gamma(t)$ defined in Eq. (3.15):

$$
\gamma^{p,+}(t)=\gamma(t), \quad \gamma^{p,-}(t)=\gamma(-t)=\gamma^{*}(t) .
$$

To derive a kinetic equation we call diagrams irreducible if any vertical cut crosses at least one dashed line. Fig. 3.4 (respectively Fig. 3.3) shows four such irreducible blocks (a)-(d). We denote the sum over all irreducible diagrams with the outermost vertices at the time points $t_{2}$ and $t_{1}\left(t_{1}>t_{2}\right)$ by the kernel $\Sigma\left(t_{1}-t_{2}\right)$. Diagrammatically, $p(t)$ is then given by all possible sequences of such kernels $\Sigma$. In Fig. 3.5 an iteration scheme for $p(t)$ is shown (the double line of the Keldysh contour is now represented by only one line). Here, $p(t)$ is represented by the thick line, and the iteration starts with the free propagation, which corresponds to the thin line. The iterative solution is formally given by the self-consistent equation

$$
p(t)=e^{-i L_{0} t} p(0)+\int_{0}^{t} d t_{1} \int_{0}^{t_{1}} d t_{2} e^{-i L_{0}\left(t-t_{1}\right)} \Sigma\left(t_{1}-t_{2}\right) p\left(t_{2}\right)
$$

with the superoperator $\Sigma$ :

$$
\Sigma\left(t_{1}-t_{2}\right)=(-i)^{2} \operatorname{Tr}_{B}\left[L_{V} e^{-i L_{0} t_{1}} T e^{-i \int_{t_{2}}^{t_{1}} d t L_{V}(t)} e^{i L_{0} t_{2}} L_{V} \rho_{B}^{e q}\right]_{\text {irred. }} .
$$

Here the index "irred." indicates that only irreducible diagrams are taken into account. Differentiating Eq. (3.28) with respect to time $t$ leads to the standard kinetic equation $[12,14]$

$$
\dot{p}(t)+i L_{0} p(t)=\int_{0}^{t} d t^{\prime} \Sigma\left(t-t^{\prime}\right) p\left(t^{\prime}\right) .
$$

The left-hand side of Eq. (3.30) describes the time evolution of the two-state system in the absence of a coupling to the bath, whereas the right-hand side contains the dissipative part which drives the reduced density matrix into a stationary state. Because of 
$\lim _{t \rightarrow \infty} p(t)=p_{s t}$ we may take the Laplace transform of $p(t)$ in the upper half plane, i.e. for $\operatorname{Im} z>0$. With

$$
p(z)=\int_{0}^{\infty} d t e^{i z t} p(t), \quad \Sigma(z)=\int_{0}^{\infty} d t e^{i z t} \Sigma(t),
$$

we get an explicit equation for $p$ :

$$
p(z)=\Pi(z) p(0), \quad \Pi(z)=\frac{i}{z-L_{0}-i \Sigma(z)} .
$$

Thus, the knowledge of $\Sigma(z)$ provides the full time evolution of the reduced density matrix. The stationary solution $p_{s t}$ follows from

$$
p_{s t}=-i \lim _{z \rightarrow 0} z p(z)
$$

This yields

$$
\left[L_{0}+i \Sigma(z=0)\right] p_{s t}=0 .
$$

For the spin-boson model we also calculate the static susceptibility $\chi_{0}$, which is defined as

$$
\chi_{0}=-\frac{d}{d \epsilon} \operatorname{Tr}_{0}\left[\sigma_{z} p_{s t}\right]
$$

where $\operatorname{Tr}_{0}$ denotes the trace over the local degrees of freedom.

\subsubsection{RTRG method}

Perturbative results for the spin-boson model (e.g. following from Redfield theory [61]) can be recovered by calculating the kernel $\Sigma(z)$ up to first order in the coupling $\alpha$. The aim of the present study, however, is to go beyond and calculate the kernel nonperturbatively by a systematic RG procedure. The idea is to integrate out all contraction lines one after another, as we have already outlined in Section 2.3. This is accounted for by both a renormalized kernel $\Sigma(z)$ and a renormalized propagator and vertices. We formally introduce the time scale $t_{c}$, which the bath contractions depend on:

$$
\gamma^{p_{1}, p_{2}}\left(t, t_{c}\right)=\gamma^{p_{1}, p_{2}}(t) q\left(t, t_{c}\right), \quad t>0 .
$$

Starting with $t_{c}=0$ the RG flow is determined by the cutoff-function $q\left(t, t_{c}\right)$. The standard choice of $q\left(t, t_{c}\right)$ is the sharp cutoff $[13,14,24]$

$$
q\left(t, t_{c}\right)=\Theta\left(t-t_{c}\right)
$$

This choice has already been applied in Section 2.3. With this form of $\gamma^{p_{1}, p_{2}}\left(t, t_{c}\right)$ the RG flow can be described within the diagrammatic language. At a fixed value of $t_{c}$ all contractions with a time scale $t<t_{c}$ have already been integrated out, which again reminds us of the remarks in Section 2.3. Thus, in Fig. 3.4 (respectively Fig. 3.3) the shortest contraction line of each of the blocks (a)-(d) is integrated out first. In the diagrams (a) 
and (b) this leads to a renormalization of $L_{0}$, while in the blocks (c) and (d) a vertex is renormalized. The contractions connecting the upper and the lower propagator (as in (b)) may change the states on the upper and the lower propagator simultaneously. This gives rise to rates, so that the renormalized Liouvillian $L_{0}$ cannot be represented by a commutator with a renormalized Hamiltonian $H_{0}$ any more, i.e. $L_{0} \neq\left[H_{0}, \cdot\right]$ for $t_{c}>0$. Thereby a non-Hamiltonian dynamics is generated. Correspondingly, due to diagrams as (d), a renormalized vertex generally acts on both the forward and backward propagator simultaneously. In the following we denote the rightmost (leftmost) vertex of the kernel $\Sigma(z)$ by $A^{p}(z)\left(B^{p}(z)\right)$. They are renormalized in a different way than $G^{p}$ and also become $z$ dependent for $t_{c}>0$. It should be emphasized that, although the definition of the cutoff-function in Eqs. (3.36) and (3.37) has the advantage that it can be understood in this rather simple picture, this choice is not necessary. Formally, one may think of an arbitrary $t_{c}$ dependence of the function $\gamma^{p_{1}, p_{2}}\left(t, t_{c}\right)$, which only has to fulfill the initial condition

$$
\gamma^{p_{1}, p_{2}}\left(t, t_{c}=0\right)=\gamma^{p_{1}, p_{2}}(t)
$$

Furthermore, a convergent RG flow, where all diagrams are integrated out, can only be expected for

$$
\lim _{t_{c} \rightarrow \infty} \gamma^{p_{1}, p_{2}}\left(t, t_{c}\right)=0
$$

Note that for the spin-boson model the above equation is fulfilled for the sharp cutoff defined in Eqs. (3.36) and (3.37), since

$$
\lim _{t \rightarrow \infty} \gamma^{p_{1}, p_{2}}(t)=0
$$

The renormalization for a general function $\gamma^{p_{1}, p_{2}}\left(t, t_{c}\right)$ is based on the invariance of $\Sigma(z)$. Before introducing $t_{c}$ the kernel $\Sigma$ is given by definition as some functional $\mathcal{F}$ :

$$
\Sigma(z)=\mathcal{F}\left(L_{0}, G^{p}, A^{p}, B^{p}, \gamma^{p_{1}, p_{2}}(t)\right)
$$

with $A^{p}=B^{p}=G^{p}$. Introducing the time scale $t_{c}$ we postulate the invariance of the left-hand side of Eq. (3.41). This can be achieved by writing

$$
\Sigma(z)=\Sigma\left(z, t_{c}\right)+\mathcal{F}\left(L_{0}\left(t_{c}\right), G^{p}\left(t_{c}\right), A^{p}\left(z, t_{c}\right), B^{p}\left(z, t_{c}\right), \gamma^{p_{1}, p_{2}}\left(t, t_{c}\right)\right) .
$$

With Eq. (3.38) the initial condition at $t_{c}=0$ is then given by Eq. (3.41):

$$
\begin{aligned}
\Sigma\left(z, t_{c}=0\right) & =0 \\
L_{0}\left(t_{c}=0\right) & =L_{0} \\
G^{p}\left(t_{c}=0\right) & =A^{p}\left(z, t_{c}=0\right)=B^{p}\left(z, t_{c}=0\right)=G^{p} .
\end{aligned}
$$

Because of Eq. (3.39) and $\mathcal{F}=\mathcal{O}\left(\gamma^{p_{1}, p_{2}}\left(t, t_{c}\right)\right)$ a solution is found for $t_{c} \rightarrow \infty$ :

$$
\Sigma(z)=\lim _{t_{c} \rightarrow \infty} \Sigma\left(z, t_{c}\right)
$$

In the following we derive the renormalization scheme determining $\Sigma\left(z, t_{c}\right)$. 
When incrementing the cutoff $t_{c} \rightarrow t_{c}+d t_{c}$, the left-hand side of Eq. (3.42) stays the same, so that

$$
\begin{aligned}
0= & d \Sigma(z)+\frac{\partial \mathcal{F}}{\partial L_{0}} d L_{0}+\sum_{p}\left(\frac{\partial \mathcal{F}}{\partial G^{p}} d G^{p}+\frac{\partial \mathcal{F}}{\partial A^{p}(z)} d A^{p}(z)+\frac{\partial \mathcal{F}}{\partial B^{p}(z)} d B^{p}(z)\right) \\
& +\sum_{p_{1}, p_{2}} \int_{0}^{\infty} d t \frac{\partial \mathcal{F}}{\partial \gamma^{p_{1}, p_{2}}\left(t, t_{c}\right)} d \gamma^{p_{1}, p_{2}}\left(t, t_{c}\right)
\end{aligned}
$$

where we have omitted the $t_{c}$ dependence of the objects $\Sigma\left(z, t_{c}\right), L_{0}\left(t_{c}\right), G^{p}\left(t_{c}\right), A^{p}\left(z, t_{c}\right)$ and $B^{p}\left(z, t_{c}\right)$. According to Eqs. (3.29) and (3.31), a term contributing to $\mathcal{F}$ has the form

$$
\left.(-i)^{n} \int_{0}^{\infty} d t_{1} \ldots \int_{0}^{t_{n-2}} d t_{n-1}{\overparen{A^{p_{1}}\left(t_{1}\right.}}_{1} z\right) \cdots \mathrm{G}^{p_{j}}\left(t_{j}\right) \ldots G^{p_{k}}\left(t_{k}\right) \cdots B^{p_{n}}(0, z),
$$

where " $\neg$ " represents a contraction. The time-dependence of $G^{p}(t), A^{p}(t, z)$ and $B^{p}(t, z)$ stems from the interaction picture respectively the Laplace transform, it is given by

$$
\begin{aligned}
G^{p}(t) & =e^{i L_{0} t} G^{p} e^{-i L_{0} t}, \\
A^{p}(t, z) & =e^{i z t} A^{p}(z) e^{-i L_{0} t}, \\
B^{p}(t, z) & =e^{i L_{0} t} B^{p}(z) e^{-i z t} .
\end{aligned}
$$

The last term in Eq. (3.45) leads to terms where one contraction line $\gamma^{p_{1}, p_{2}}\left(t, t_{c}\right)$ is re-

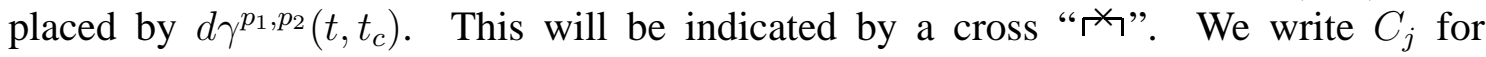
$G_{j} \equiv G^{p_{j}}\left(t_{j}\right)$ respectively $A_{j} \equiv A^{p_{j}}\left(t_{j}, z\right)$ or $B_{j} \equiv B^{p_{j}}\left(t_{j}, z\right)$ and define a "cross contraction" by

$$
\overparen{C_{j} \cdots C_{k}}=-\frac{d \gamma^{p_{j}, p_{k}}}{d t_{c}}\left(t_{j}-t_{k}, t_{c}\right) d t_{c} C_{j} \cdots C_{k} .
$$

We have included a minus sign in Eq. (3.47) in order to later identify a cross contraction with the renormalization contributions $d \Sigma(z), d L_{0}, d G^{p}, d A^{p}(z), d B^{p}(z)$, so that Eq. (3.45) is fulfilled.

Let us now consider terms of the form

$$
\int_{t_{1}>t_{2}>t_{3}>t_{4}} d t_{2} d t_{3} C_{1} \vec{G}_{2}^{*} G_{3} C_{4}
$$

Such terms contribute to a renormalization of $L_{0}$, which corresponds to the second term in Eq. (3.45). The propagation at a given time point $t^{*}$ with $t_{1}>t^{*}>t_{4}$ for an infinitesimal time interval $d t$ can be expanded

$$
\exp \left(-i L_{0} d t\right)=\mathbb{1}-i L_{0} d t
$$

Thus, the second term in Eq. (3.45) gives rise to a contribution $d L_{0}\left(t^{*}\right)=e^{i L_{0} t^{*}} d L_{0} e^{-i L_{0} t^{*}}$ to the renormalized Liouvillian at any time point $t^{*}$. Regarding the above term (3.48) one 
has to choose this time point $t^{*}$, which the cross contraction corresponds to. We fix that time ordering variable $t^{*}$ at the smaller time point of the cross contraction. To do that formally the term (3.48) is written as

$$
\begin{aligned}
& \int_{t_{1}>t_{2}>t_{3}>t_{4}} d t_{2} d t_{3} C_{1} \stackrel{\boldsymbol{K}_{4}}{G_{2}} G_{3} C_{4}=\int_{\substack{t_{1}>t_{3}>t_{4} \\
t_{2}>t_{3}}} d t_{2} d t_{3} C_{1} \stackrel{\boldsymbol{K}_{7}}{G_{2}} G_{3} C_{4} \\
& -\int_{t_{2}>t_{1}>t_{3}} d t_{2} d t_{3} C_{1} \stackrel{\Gamma^{\star}}{G_{2}} G_{3} C_{4} \\
& +\int_{t_{2}>t_{1}>t_{4}>t_{3}} d t_{2} d t_{3} C_{1} \stackrel{\boldsymbol{K}_{2}}{G_{2}} G_{3} C_{4}
\end{aligned}
$$

The first term on the right-hand side of Eq. (3.50) is identified with $d L_{0}\left(t_{3}\right)$. Including the factors $(-i)$ and the sums over $p_{2}, p_{3}$ we obtain

$$
-i d L_{0}\left(t_{3}\right)=(-i)^{2} \int_{t_{3}}^{\infty} d t_{2} \sum_{p_{2}, p_{3}} \stackrel{\Gamma}{*}_{2} G_{3}
$$

Shifting the integration boundaries leads to

$$
-i d L_{0}(0)=(-i)^{2} \int_{0}^{\infty} d t \sum_{p_{1}, p_{2}} \stackrel{G^{p_{1}}(t)}{x} G^{p_{2}}(0) .
$$

The second term on the right-hand side of Eq. (3.50) is a correction term due to the choice of the time ordering. Its occurence can be understood, if one multiplies $d L_{0}\left(t_{3}\right)$ from the left by some vertex $C_{1}$. Then time ordering requires $t_{1}>t_{3}$. Thus, $t_{1}$ is decoupled from the integration variable $t_{2}$, and terms with $t_{2}>t_{1}>t_{3}$ occur. Since such diagrams have not been present before calculating $d L_{0}$, we have to substract the corresponding correction term in Eq. (3.50). It is interpreted as a contribution to a renormalized vertex $C_{1}$, where $C_{1}=G_{1}$ respectively $C_{1}=A_{1}$. Since $B$ is the vertex with the smallest time argument in $\Sigma(z), C_{1}=B_{1}$ is not allowed. Thus, we account for this term using the third (respectively fourth) term in Eq. (3.45). In these terms the vertex $G^{p}\left(A^{p}\right)$ is replaced by $d G^{p}\left(d A^{p}\right)$. Denoting the correction term by $d C_{1}^{(c)}$ we obtain

$$
-i d C_{1}^{(c)}=-(-i)^{3} \int_{t_{2}>t_{1}>t_{3}} d t_{2} d t_{3} \sum_{p_{2}, p_{3}} C_{1} \stackrel{\mathrm{G}_{2}}{G_{3}} .
$$

The last term on the right-hand side of Eq. (3.50) is interpreted as a double vertex, since both $t_{1}$ and $t_{4}$ lie within the contraction interval $\left[t_{2}, t_{3}\right]$. Such objects are neglected. A further vertex renormalization stems from terms of the form

$$
\int_{t_{2}>t_{1}>t_{3}} d t_{2} d t_{3} \stackrel{\times}{C_{2} G_{1} G_{3}} .
$$


If $C_{2}=G_{2}$ this contributes to $d G_{1}$, whereas $C_{2}=A_{2}$ leads to a renormalization $d A_{1}$. Correspondingly we obtain a contribution $d B_{1}$ from

$$
\int_{t_{2}>t_{1}>t_{3}} d t_{2} d t_{3} \stackrel{\stackrel{\times}{G}_{2} G_{1} B_{3}}{.}
$$

The terms (3.54) and (3.55) again cause correction terms. However, they correspond to double and higher-order vertex objects, which are neglected. Thus, with shifted integration boundaries the total renormalization contributions $d G^{p}, d A^{p}$ and $d B^{p}$ read

$$
\begin{aligned}
& -i d G^{p}(0)=(-i) \int_{\infty>t>0>t^{\prime}>-\infty} d t d t^{\prime} \sum_{p_{1}, p_{2}}\left(G^{p}(0) \stackrel{\gtrless}{G^{p_{1}}(t)} G^{p_{2}}\left(t^{\prime}\right)\right. \\
& -\overbrace{G^{p_{1}}(t) G^{p}(0)}^{\times} G^{p_{2}}\left(t^{\prime}\right)) \\
& -i d A^{p}(0, z)=(-i) \int_{\infty>t>0>t^{\prime}>-\infty} d t d t^{\prime} \sum_{p_{1}, p_{2}}\left(A^{p}(0, z) \stackrel{\overrightarrow{G^{p_{1}}(t)} G^{p_{2}}}{\left.t^{\prime}\right)}\right. \\
& -\overbrace{A^{p_{1}}(t, z) G^{p}(0)}^{\times} G^{p_{2}}\left(t^{\prime}\right)) \text {, } \\
& -i d B^{p}(0, z)=-(-i) \int_{\infty>t>0>t^{\prime}>-\infty} d t d t^{\prime} \sum_{p_{1}, p_{2}} \stackrel{G^{p_{1}}(t) G^{p}(0) B^{p_{2}}}{\left.t^{\prime}, z\right) .}
\end{aligned}
$$

The renormalization scheme is completed by the terms connecting boundary vertex objects. The terms

$$
\stackrel{\star^{*}}{A_{1} G_{2}} \quad \text { and } \quad \stackrel{*^{*}}{G_{1} B_{2}}
$$

do not occur, since we consider only irreducible diagrams. The remaining term

$$
\stackrel{\aleph^{\prime}}{A_{1}}
$$

is accounted for by the renormalization $d \Sigma(z)$ in Eq. (3.45). With the definition of the Laplace transform this yields

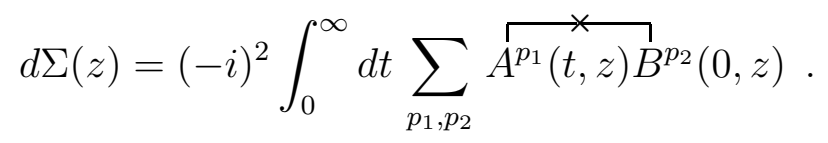

Using the definition in Eq. (3.47), the renormalization in Eq. (3.52) and Eqs. (3.56) - 
(3.59) leads to the $\mathrm{RG}$ equations

$$
\begin{aligned}
\frac{d L_{0}}{d t_{c}} & =i \int_{0}^{\infty} d t \sum_{p_{1}, p_{2}} \frac{d \gamma^{p_{1}, p_{2}}}{d t_{c}}\left(t, t_{c}\right) G^{p_{1}}(t) G^{p_{2}}(0), \\
\frac{d G^{p}}{d t_{c}} & =\int_{0}^{\infty} d t \int_{-\infty}^{0} d t^{\prime} \sum_{p_{1}, p_{2}} \frac{d \gamma^{p_{1}, p_{2}}}{d t_{c}}\left(t-t^{\prime}, t_{c}\right) \\
& \times\left(G^{p_{1}}(t) G^{p}(0)-G^{p}(0) G^{p_{1}}(t)\right) G^{p_{2}}\left(t^{\prime}\right), \\
\frac{d A^{p}}{d t_{c}}(z) & =\int_{0}^{\infty} d t \int_{-\infty}^{0} d t^{\prime} \sum_{p_{1}, p_{2}} \frac{d \gamma^{p_{1}, p_{2}}}{d t_{c}}\left(t-t^{\prime}, t_{c}\right) \\
\frac{d B^{p}}{d t_{c}}(z) & \left.=\int_{0}^{\infty} d t \int_{-\infty}^{0} d A^{p_{1}}(t, z) G^{p}(0)-A^{p}(0, z) G^{p_{1}}(t)\right) G^{p_{2}}\left(t^{\prime}\right), \\
\frac{d \Sigma}{d t_{c}}(z) & =\int_{0}^{\infty} d t \sum_{p_{1}, p_{2}} \frac{d \gamma^{p_{1}, p_{2}}}{d t_{c}}\left(t-t^{\prime}, t_{c}\right)
\end{aligned}
$$

The $t_{c}$ dependence of the function $\gamma^{p_{1}, p_{2}}\left(t, t_{c}\right)$ will be chosen such that one integral on the right-hand sides of the above equations becomes trivial (e.g. the sharp cutoff given in Eqs. (3.36) and (3.37)). In principle, the integration variables only refer to the interaction picture, so that we deal with pure differential equations.

\subsubsection{Remarks}

The first order contribution in $\alpha$ to the kernel $\Sigma(z)$ may be obtained by neglecting the $t_{c}$ dependence of $L_{0}, A^{p}(z)$ and $B^{p}(z)$, i.e. integrating only Eq. (3.64). In the set of ordinary differential equations (3.60) - (3.64) double and higher-order vertex objects have been neglected. Principally the RG scheme allows a systematic study of all higher-order vertex objects - the additional terms arising from double vertex objects are shown in Appendix C. However, the number of terms increases substantially, and due to retardation effects of the double vertices one deals with integro-differential equations. Thus, accounting for double vertex objects within this formalism increases the numerical effort rapidly. However, in Chapter 5 we will present a formulation of the RTRG, where double vertices can also be included. At this stage we expect from the approximation a restriction in the magnitude of the coupling constant $\alpha$, since double vertex objects are of first order in $\gamma(t)$ respectively $\alpha$. In fact, within Eqs. (3.60) - (3.64) the kernel $\Sigma(z)$ is contained exactly up to second order in $\alpha$. The explicit range of validity of the approximation cannot be derived directly from the formalism, but for the spin-boson model we will find that our results are accurate for $\alpha \lesssim 0.1-0.2$. In this context note, that although third and higher orders of $\alpha$ are not contained exactly within the approximation, by integrating 
Eqs. (3.60) - (3.64) we still account for diagrams of all orders of $\alpha$. Thus, the RG equations naturally account for nonperturbative effects; for the spin-boson model we will calculate the correct renormalized tunnel amplitude $\Delta_{r}$ in Section 3.4.1. Furthermore, because we have not expanded the propagator in Eqs. (3.60) - (3.64), all time scales are considered and we do not deal with any restriction regarding the eigenvalues of $L_{0} t_{c}$. Thus, in contrast to a Poor Man's scaling approach (see Eqs. (3.9) and (3.10)) we do not have to stop the RG flow at a finite $t_{c}$ (respectively $\omega_{c}$ in Eqs. (3.9) and (3.10)), but we may consider the limit of $t_{c} \rightarrow \infty$.

The initial values of the superoperators are given by Eq. (3.43). For the spin-boson model we obtain from Eqs. (3.21) and (3.24) for the corresponding matrices

$$
\begin{aligned}
\left(L_{0}\right)_{s_{1} s_{1}^{\prime}, s_{2} s_{2}^{\prime}} & =\delta_{s_{1}^{\prime}, s_{2}^{\prime}}\left(H_{0}\right)_{s_{1} s_{2}}-\delta_{s_{1}, s_{2}}\left(H_{0}\right)_{s_{2}^{\prime} s_{1}^{\prime}} \\
\left(G^{+}\right)_{s_{1} s_{1}^{\prime}, s_{2} s_{2}^{\prime}} & =\delta_{s_{1}^{\prime}, s_{2}^{\prime}}\left(\frac{\sigma_{z}}{2}\right)_{s_{1} s_{2}}, \\
\left(G^{-}\right)_{s_{1} s_{1}^{\prime}, s_{2} s_{2}^{\prime}} & =-\delta_{s_{1}, s_{2}}\left(\frac{\sigma_{z}}{2}\right)_{s_{2}^{\prime} s_{1}^{\prime}} .
\end{aligned}
$$

Due to Hermiticity of the Hamiltonian it follows

$$
\begin{aligned}
\left(i L_{0}\right)_{s_{1} s_{1}^{\prime}, s_{2} s_{2}^{\prime}} & =\left(i L_{0}\right)_{s_{1}^{\prime} s_{1}, s_{2}^{\prime} s_{2}}^{*}, \\
\left(i G^{p}\right)_{s_{1} s_{1}^{\prime}, s_{2} s_{2}^{\prime}} & =\left(i G^{\bar{p}}\right)_{s_{1}^{\prime} s_{1}, s_{2}^{\prime} s_{2}}^{*},
\end{aligned}
$$

where $\bar{p}=\mp$ for $p= \pm$. Using Eq. (3.27) one finds that the above symmetry is conserved by the flow equations (3.60) - (3.64). Thus, the symmetry relations (3.68) and (3.69) are valid for all $t_{c} \geq 0$, and we also have

$$
\begin{aligned}
\left(i A^{p}(z)\right)_{s_{1} s_{1}^{\prime}, s_{2} s_{2}^{\prime}} & =\left(i A^{\bar{p}}\left(-z^{*}\right)\right)_{s_{1}^{\prime} s_{1}, s_{2}^{\prime} s_{2}}^{*} \\
\left(i B^{p}(z)\right)_{s_{1} s_{1}^{\prime}, s_{2} s_{2}^{\prime}} & =\left(i B^{\bar{p}}\left(-z^{*}\right)\right)_{s_{1}^{\prime} s_{1}, s_{2}^{\prime} s_{2}}, \\
(\Sigma(z))_{s_{1} s_{1}^{\prime}, s_{2} s_{2}^{\prime}} & =\left(\Sigma\left(-z^{*}\right)\right)_{s_{1}^{\prime} s_{1}, s_{2}^{\prime} s_{2}} .
\end{aligned}
$$

From Eq. (3.32) one then concludes

$$
p(z)_{s s^{\prime}}=\left(p\left(-z^{*}\right)\right)_{s^{\prime} s}^{*}
$$

for $p(0)$ being Hermitian. Thus, the RG equations conserve the Hermiticity of $p(t)$. Furthermore,

$$
\begin{aligned}
0 & =\sum_{s}\left(L_{0}\right)_{s s, s_{1} s_{2}}=\sum_{s, p}\left(G^{p}\right)_{s s, s_{1} s_{2}} \\
& =\sum_{s, p}\left(A^{p}(z)\right)_{s s, s_{1} s_{2}}=\sum_{s, p}\left(B^{p}(z)\right)_{s s, s_{1} s_{2}}=\sum_{s}(\Sigma(z))_{s s, s_{1} s_{2}}
\end{aligned}
$$

is fulfilled for all $t_{c} \geq 0$. With the kinetic equation (3.30) one then finds the conservation of probability: $d / d t \operatorname{Tr}_{0} p(t)=0$. Eqs. (3.74) also show that a solution of Eq. (3.34) for 
the stationary reduced density matrix $p_{s t}$ exists, since the sum of two rows of the matrix $L_{0}+i \Sigma(z=0)$ vanishes. Furthermore, because of Eqs. (3.68) and (3.72) this solution can be chosen to be Hermitian. However, the eigenvalues $\lambda_{i}$ of $p_{s t}$ do not necessarily satisfy the relation $0 \leq \lambda_{i} \leq 1$ for all $t_{c}$.

Finally, we note that the scheme we used for the polaron in Section 2.3 can be recovered from Eqs. (3.60) and (3.61) by taking only the upper propagator, i.e. $p=p_{1}=p_{2}=+$, and choosing the sharp cutoff defined in Eqs. (3.36) and (3.37). To obtain Eqs. (2.54) and (2.55) we have to replace the superoperators by the corresponding operators: $L_{0} \rightarrow H_{0}$ and $G^{p} \rightarrow G_{q}$. Note that in case of the polaron we had a $q$ dependent operator $G_{q}$ in the electron system (see Eq. (2.53)). In the above language this means that also the bath operator $j$ becomes $q$ dependent. Thus, in such a situation the bath contraction $\gamma$ formally depends on two indices $q_{1}$ and $q_{2}$, which the righthand sides of the RG equations are then summed over. In case of the polaron we had $\gamma_{q_{1}, q_{2}}=\delta_{q_{1},-q_{2}} \exp (-i \omega t)$. From this contraction there arose difficulties concerning the convergence, because Eq. (3.39) was not fulfilled, since $\exp (-i \omega t)$ does not decay. Furthermore, for the polaron we were not able to use the RTRG formalism on the Keldysh contour, as the continuous electron spectrum led to a large size of the numerical problem. Additionally, we had to perform the sums over the bath degrees of freedom numerically. In contrast, the spin-boson model has only two degrees of freedom in the local system and the bath degrees of freedom have been integrated out analytically when calculating $\gamma(t)$. Thus, for the spin-boson model we are able to use the full nonequilibrium version of the RTRG, where we work on the Keldysh contour. We point out that already in case of the polaron we accounted for level broadening respectively finite life-times, since the energies became complex. On the Keldysh contour this physics is included by contraction lines connecting vertices, which lie only on one propagator, i.e. $p_{1}=p_{2}$ in $\gamma^{p_{1}, p_{2}}$. The complex energies already lead to the inequality $L_{0} \neq\left[H_{0}, \cdot\right]$ for $t_{c}>0$ [12]. This however does not reflect rates invoked by dissipation. As explained in the preceding subsection this phenomenon corresponds to diagrams, which connect the upper and the lower propagator, since these terms may change the states on both propagators simultaneously. Thereby the system is driven irreversibly into a stationary state.

\subsubsection{Equilibrium correlation functions}

For the calculation of equilibrium correlation functions the formalism explained above is not sufficient, because then initial correlations are important. They did not occur above, since we assumed a factorized initial state. In the following we develop a generalization of the method, which accounts for such initial correlations. Let us consider an equilibrium correlation function of the form

$$
C(t)=\operatorname{Tr}\left[[g(t), d]_{ \pm} \rho^{e q}\right] .
$$

Here, $g$ and $d$ are some operators for the local system and $[\cdot, \cdot]_{ \pm}$denotes the anticommutator respectively the commutator. This can be written as

$$
C(t)=\operatorname{Tr}\left[g e^{-i L t} D \rho^{e q}\right]
$$




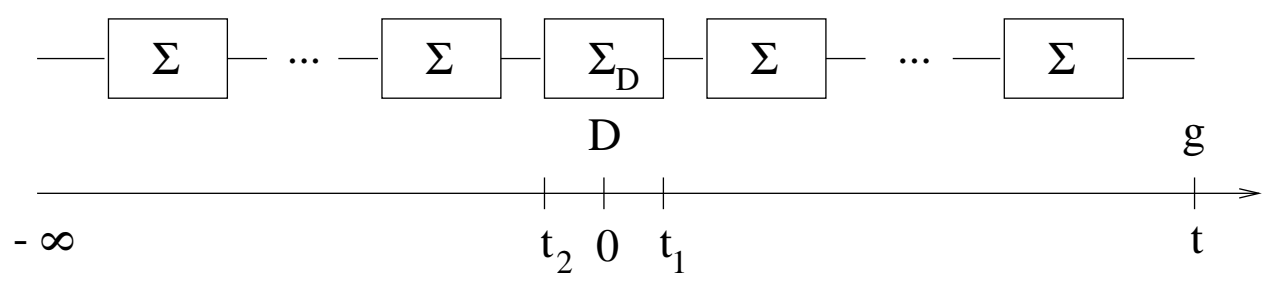

Figure 3.6: The diagrammatic expression for an equilibrium correlation function $\mathrm{C}(\mathrm{t})$, when $D$ lies within a $\Sigma$ block.

with the superoperator $D^{1}$. It acts on an operator $O$ by

$$
D O=d O \pm O d
$$

where the sign depends on whether we consider the correlation function for the anticommutator or the commutator of $g$ and $d$. The density matrix in equilibrium $\rho^{e q}$ can be expressed by an evolution out of a factorized initial state at $t_{0} \rightarrow-\infty$. Thus

$$
C(t)=\lim _{t_{0} \rightarrow-\infty} \operatorname{Tr}_{0} \operatorname{Tr}_{B}\left[g e^{-i L t} D e^{-i \int_{t_{0}}^{0} d t^{\prime} L} p\left(t_{0}\right) \rho_{B}^{e q}\right]
$$

To perform the trace over the bath degrees of freedom we proceed analogously to the dicussion of the reduced density matrix. The diagrammatic expression for Eq. (3.78) is again given by all possible sequences of $\Sigma$ blocks of irreducible diagrams. However, in this case the superoperator $D$ may lie within such a block, i.e. $D$ may be crossed by at least one contraction line. This gives rise to a new object, which we call $\Sigma_{D}[25,26]$ (see Fig. 3.6). It depends not only on the time difference between the last and the first vertex $\left(t_{1}-t_{2}\right)$, but also on the time difference between $D$ and the first vertex $\left(0-t_{2}\right)$. We write $\Sigma_{D}=\Sigma_{D}\left(t_{1}-t_{2},-t_{2}\right)$. To evaluate the diagrams in Fig. 3.6 we take the Laplace inversion of Eq. (3.32). This gives us an equation for the evolution of the reduced density matrix out of a factorized state at $t=0$ :

$$
p(t)=\Pi(t) p(0)
$$

Thus, the superoperator $\Pi(t)$ accounts for all sequences of $\Sigma$ blocks in a time interval with length $t$. We may now express $C(t)$ by superoperators acting only in the local system. With $\lim _{t_{0} \rightarrow-\infty} \Pi\left(t_{1}-t_{0}\right) p\left(t_{0}\right)=p_{s t}$ the total result for $C(t)$ reads

$$
C(t)=\operatorname{Tr}_{0}\left[g \Pi(t) D p_{s t}+\int_{0}^{t} d t_{1} \int_{-\infty}^{0} d t_{2} g \Pi\left(t-t_{1}\right) \Sigma_{D}\left(t_{1}-t_{2},-t_{2}\right) p_{s t}\right],
$$

where the first term accounts for the diagrams, where $D$ is not crossed by any contraction line. Switching back to Laplace space yields

$$
C(z)=\operatorname{Tr}_{0}\left[g \Pi(z)\left(D+\Sigma_{D}(z)\right) p_{s t}\right]
$$

\footnotetext{
${ }^{1}$ It is clear from the context, whether $D$ refers to this superoperator or the high-energy cutoff.
} 
with $C(z)=\int_{0}^{\infty} d t e^{i z t} C(t), \Pi(z)$ as in Eq. (3.32) and

$$
\begin{aligned}
\Sigma_{D}(z) & =\int_{0}^{\infty} d t_{1} \int_{-\infty}^{0} d t_{2} e^{i z t_{1}} \Sigma_{D}\left(t_{1}-t_{2},-t_{2}\right) \\
& =\int_{0}^{\infty} d t_{1} \int_{0}^{\infty} d t_{2} e^{i z t_{1}} \Sigma_{D}\left(t_{1}+t_{2}, t_{2}\right) \\
& =\int_{0}^{\infty} d t e^{i z t} \int_{0}^{t} d t^{\prime} e^{-i z t^{\prime}} \Sigma_{D}\left(t, t^{\prime}\right)
\end{aligned}
$$

While $\Pi(z)$ only depends on $\Sigma(z)$, and therefore can be calculated from Eqs. (3.60) (3.64), we have to set up additional RG equations for determining $\Sigma_{D}$. Formally, it is given by

$$
\Sigma_{D}\left(t, t^{\prime}\right)=(-i)^{2} \operatorname{Tr}_{B}\left[L_{V} e^{-i L_{0} t} T e^{-i \int_{0}^{t} d t L_{V}(t)} D\left(t^{\prime}\right) L_{V} \rho_{B}^{e q}\right]_{\text {irred. }},
$$

where $T$ again is the time ordering operator, and the interaction picture with respect to $L_{0}+L_{B}$ has been used.

The renormalization scheme for this object can be derived within the same framework as in Subsection 3.2.2. We obtain

$$
d \Sigma_{D}(z)=(-i)^{2} \int_{0}^{\infty} d t \int_{0}^{t} d t^{\prime} \sum_{p_{1}, p_{2}} \stackrel{A_{A^{p_{1}}(t, z) D\left(t^{\prime}\right)}^{x}}{B^{p_{2}}}(0) .
$$

According to the Laplace integral in Eq. (3.82) the objects $D$ and $\bar{B}$ are defined by

$$
\begin{aligned}
D(t) & =e^{i L_{0} t} D e^{-i L_{0} t} e^{-i z t}, \\
\bar{B}^{p}(t) & =B^{p}(t, z=0)=e^{i L_{0} t} B^{p}(z=0) .
\end{aligned}
$$

In the following we will interpret $D$ as a vertex. Thus, the renormalization of $D$ is analogous to Eq. (3.61). We therefore end up with two additional RG equations for the calculation of equilibrium correlation functions. They read

$$
\begin{aligned}
\frac{d D}{d t_{c}}=\int_{0}^{\infty} d t \int_{-\infty}^{0} d t^{\prime} \sum_{p_{1}, p_{2}} \frac{d \gamma^{p_{1}, p_{2}}}{d t_{c}}\left(t-t^{\prime}, t_{c}\right) \\
\quad \times\left(G^{p_{1}}(t) D(0)-D(0) G^{p_{1}}(t)\right) G^{p_{2}}\left(t^{\prime}\right) \\
\frac{d \Sigma_{D}}{d t_{c}}(z)=\int_{0}^{\infty} d t \int_{0}^{t} d t^{\prime} \sum_{p_{1}, p_{2}} \frac{d \gamma^{p_{1}, p_{2}}}{d t_{c}}\left(t, t_{c}\right) \\
\times A^{p_{1}}(t, z) D\left(t^{\prime}\right) B^{p_{2}}(0, z=0) .
\end{aligned}
$$

Eqs. (3.60) - (3.64) and the above equations now define the complete RG scheme. Since we treated $D$ as a vertex, we interpreted terms like

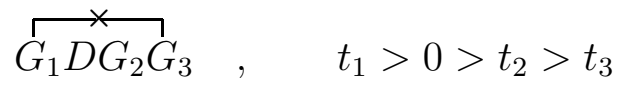


as double vertex objects, which are neglected (compare with the last term on the righthand side of Eq. (3.50)). However, if one considers the definitions of $\Sigma_{D}$ and $\Sigma$, Eqs. (3.83) and (3.29), this approximation implies that for $\Sigma_{D}$ the $\mathrm{RG}$ equations do not account for the same class of diagrams as for $\Sigma$, i.e. in contrast to $\Sigma, \Sigma_{D}$ is not contained exactly up to second order of $\alpha$.

At the end of this section we again point out that the RTRG formalism explained here may be applied to any quantum dissipative system. However, the approximation we introduced here is feasible, only if the interaction term is linear in the bath field operators. When studying problems involving a fermionic environment, one has to account for additional signs. This will be explained in detail in Chapter 4, where we study coupled quantum dots.

\subsection{RG equations for the spin-boson model}

\subsubsection{Initial values}

For the spin-boson model the initial values for the superoperators in Eq. (3.65) - (3.67) correspond to the $(4 \times 4)$-matrices

$$
\begin{aligned}
L_{0} & =\left(\begin{array}{cccc}
\epsilon & 0 & \frac{\Delta}{2} & -\frac{\Delta}{2} \\
0 & -\epsilon & -\frac{\Delta}{2} & \frac{\Delta}{2} \\
\frac{\Delta}{2} & -\frac{\Delta}{2} & 0 & 0 \\
-\frac{\Delta}{2} & \frac{\Delta}{2} & 0 & 0
\end{array}\right), \\
G^{+} & =\frac{1}{2}\left(\begin{array}{cccc}
1 & 0 & 0 & 0 \\
0 & -1 & 0 & 0 \\
0 & 0 & 1 & 0 \\
0 & 0 & 0 & -1
\end{array}\right), \\
G^{-} & =-\frac{1}{2}\left(\begin{array}{cccc}
-1 & 0 & 0 & 0 \\
0 & 1 & 0 & 0 \\
0 & 0 & 1 & 0 \\
0 & 0 & 0 & -1
\end{array}\right) .
\end{aligned}
$$

Here, we identified an arbitrary superoperator $S$ of the two-state system with a $(4 \times 4)$ matrix using the prescription

$$
S=\left(\begin{array}{cccc}
S_{u d, u d} & S_{u d, d u} & S_{u d, u u} & S_{u d, d d} \\
S_{d u, u d} & S_{d u, d u} & S_{d u, u u} & S_{d u, d d} \\
S_{u u, u d} & S_{u u, d u} & S_{u u, u u} & S_{u u, d d} \\
S_{d d, u d} & S_{d d, d u} & S_{d d, u u} & S_{d d, d d}
\end{array}\right)
$$

where the matrix elements are labeled by the two states $|u\rangle$ and $|d\rangle$. 


\subsubsection{The $t_{c}$ dependence of the bath contraction}

Let us now make an explicit choice for the $t_{c}$ dependence of $\gamma^{p_{1}, p_{2}}\left(t, t_{c}\right)$ for the RG scheme in Eqs. (3.60) - (3.64). As we have already mentioned, one possible choice is the sharp cutoff, which we want to consider first. We define

$$
\begin{aligned}
\gamma_{1}^{p_{1}, p_{2}}\left(t, t_{c}\right) & =\gamma^{p_{1}, p_{2}}(t) \Theta\left(t-t_{c}\right) \\
& =\left(R(t)+i p_{2} S(t)\right) \Theta\left(t-t_{c}\right)
\end{aligned}
$$

where we used Eq. (3.27). For $D \gg T$ the functions $R(t)$ and $S(t)$ are given by Eqs. (3.20) and (3.19). This leads to the RG equations

$$
\begin{aligned}
& \frac{d L_{0}}{d t_{c}}=-i \sum_{p_{1}, p_{2}}\left(R\left(t_{c}\right)+i p_{2} S\left(t_{c}\right)\right) G^{p_{1}}\left(t_{c}\right) G^{p_{2}}(0), \\
& \frac{d G^{p}}{d t_{c}}=-\int_{0}^{t_{c}} d t \sum_{p_{1}, p_{2}}\left(R\left(t_{c}\right)+i p_{2} S\left(t_{c}\right)\right) \\
& \times\left.\quad G^{p_{1}}(t) G^{p}(0)-G^{p}(0) G^{p_{1}}(t)\right) G^{p_{2}}\left(t-t_{c}\right), \\
& \frac{d A^{p}}{d t_{c}}(z)=-\int_{p_{1}, p_{2}}^{t_{c}} d t \sum_{\times}\left(R\left(t_{c}\right)+i p_{2} S\left(t_{c}\right)\right) \\
& \frac{d B^{p}}{d t_{c}}(z)=-\int_{0}^{t_{c}} d t \sum_{p_{1}, p_{2}}\left(R\left(t_{c}\right)+i p_{2} S\left(t_{c}\right)\right) \\
& \times G^{p_{1}}(t) G^{p}(0) B^{p_{2}}\left(t-t_{c}, z\right), \\
& \frac{d \Sigma}{d t_{c}}(z)=-\sum_{p_{1}, p_{2}}\left(R\left(t_{c}\right)+i p_{2} S\left(t_{c}\right)\right) A^{p_{1}}\left(t_{c}, z\right) B^{p_{2}}(0, z) .
\end{aligned}
$$

However, this set of equations generates unphysical terms, when calculating $\Sigma(z)$. This can easily be seen, if we restrict ourselves to the "trivial" case, where $\Delta=\epsilon=T=0$, i.e. we deal with two decoupled shifted harmonic oscillators. First, one recognizes that $L_{0}, G^{p}, A^{p}$ and $B^{p}$ commute for all $t_{c}$, since the commutator $\left[G^{+}, G^{-}\right]_{-}$and $L_{0}$ vanish initially. With

$$
M=G^{+}+G^{-}=\left(\begin{array}{cccc}
1 & 0 & 0 & 0 \\
0 & -1 & 0 & 0 \\
0 & 0 & 0 & 0 \\
0 & 0 & 0 & 0
\end{array}\right)
$$

and the identity $M G^{+}=M G^{-}$the solutions for $L_{0}$ and $G^{p}$ then read

$$
\begin{aligned}
L_{0}\left(t_{c}\right) & =-i 2 \alpha \frac{t_{c}}{t_{c}^{2}+1 / D^{2}} M^{2} \\
G^{p}\left(t_{c}\right) & =G^{p}
\end{aligned}
$$


Here, we used the result for $R(t)$ for vanishing temperature:

$$
R(t)=-2 \alpha \operatorname{Re}\left[\frac{1}{(t-i / D)^{2}}\right] .
$$

Setting $z=0$ the integrals in Eqs. (3.95) and (3.96) give rise to terms

$$
\begin{aligned}
f\left(t_{c}\right) & =\frac{e^{-i L_{0} t_{c}}-1}{-i L_{0} t_{c}} \\
& =\frac{e^{-2 \alpha \frac{t_{c}^{2}}{t_{c}^{2}+1 / D^{2}}}-1}{-2 \alpha \frac{t_{c}^{2}}{t_{c}^{2}+1 / D^{2}}},
\end{aligned}
$$

where we used $M^{4}=M^{2}$. We introduce $A=A^{+}+A^{-}$and $B=B^{+}+B^{-}$and obtain the differential equations

$$
\begin{aligned}
& \frac{d A}{d t_{c}}(z=0)=-R\left(t_{c}\right)\left(t_{c} f\left(t_{c}\right)-t_{c}\right) M^{2} A \\
& \frac{d B}{d t_{c}}(z=0)=-R\left(t_{c}\right) t_{c} f\left(t_{c}\right) M^{2} B,
\end{aligned}
$$

where, for the latter equation, we used

$$
\frac{d\left(B^{+}-B^{-}\right)}{d t_{c}}=0
$$

We now define

$$
F\left(t_{c}\right)=\int_{0}^{t_{c}} d t R(t) t f(t)
$$

so that the solutions for $A$ and $B$ read

$$
\begin{aligned}
& A\left(z=0, t_{c}\right)=M \exp \left(-F\left(t_{c}\right)+\int_{0}^{t_{c}} d t R(t) t\right), \\
& B\left(z=0, t_{c}\right)=M \exp \left(-F\left(t_{c}\right)\right) .
\end{aligned}
$$

With that we obtain from Eq. (3.97)

$$
\begin{aligned}
\frac{d \Sigma}{d t_{c}}(z=0) & =-M^{2} R\left(t_{c}\right) \exp \left(-2 F\left(t_{c}\right)-\frac{2 \alpha t_{c}^{2}}{t_{c}^{2}+1 / D^{2}}+\int_{0}^{t_{c}} d t R(t) t\right) \\
& =-M^{2} R\left(t_{c}\right) \exp \left(-2 F\left(t_{c}\right)+\alpha \ln \left(\frac{1}{\left(D t_{c}\right)^{2}+1}\right)\right) .
\end{aligned}
$$

We integrate that formally, using the substitutions $x=D t$ and $z=1 /\left(1+x^{2}\right)$ :

$$
\Sigma\left(z=0, t_{c}\right)=-2 \alpha M^{2} D \int_{0}^{D t_{c}} d x\left(2 z^{2}-z\right) e^{-2 F(x / D)+\alpha \ln z} .
$$


Expanding the above expression in $\alpha$ yields

$$
\begin{aligned}
\Sigma\left(z=0, t_{c}\right)= & -2 M^{2} \frac{t_{c}}{t_{c}^{2}+1 / D^{2}}\left[\alpha+\alpha^{2}\left(-\ln z_{c}+2 z_{c}-2\right)\right. \\
& \left.+\alpha^{3}\left(\frac{1}{2} \ln ^{2} z_{c}+\left(-2 z_{c}+4\right) \ln z_{c}+\frac{10}{3} z_{c}^{2}-\frac{25}{3} z_{c}+\frac{9}{2}\right)\right] \\
& -\alpha^{3} M^{2} D \arctan \left(D t_{c}\right)+\mathcal{O}\left(\alpha^{4}\right)
\end{aligned}
$$

where we wrote $z_{c}=1 /\left(1+\left(D t_{c}\right)^{2}\right)$. One notices that, in third order of $\alpha$ we obtain a term depending linearly on $D$. However, it is known, that for $D \rightarrow \infty$ the exact solution involves only logarithmic divergencies $[1,27]$. Thus, the choice of the sharp cutoff generates unphysical terms. Within our approximation they only arise in third or higher order of $\alpha$, since Eqs. (3.60) - (3.64) give the exact result for $\Sigma(z)$ up to second order in $\alpha$.

In the following the generation of unphysical terms is avoided by making an appropriate choice for the $t_{c}$ dependence of $\gamma^{p_{1}, p_{2}}\left(t, t_{c}\right)$. We introduce

$$
\gamma^{p_{1}, p_{2}}\left(t, t_{c}\right)=\frac{d}{d t}\left(\int_{0}^{t} d t^{\prime} R\left(t^{\prime}\right) \Theta\left(t-t_{c}\right)\right)+i p_{2} S(t) \Theta\left(t-t_{c}\right)
$$

Compared to the sharp cutoff, this choice includes an additional term stemming from the derivative of the $\Theta$ function. From Eq. (3.20) we obtain

$$
\int_{0}^{t} d t^{\prime} R\left(t^{\prime}\right)=2 \alpha \operatorname{Re}[\pi T \operatorname{coth}(\pi T(t-i / D))]
$$

The asymptotic behaviour is given by $\int_{0}^{\infty} d t^{\prime} R\left(t^{\prime}\right)=2 \alpha \pi T$. Therefore the above definition does not satisfy Eq. (3.39). However, we may generate a convergent RG flow by modifying Eq. (3.112). We write

$$
\tilde{R}(t)=\int_{0}^{t} d t^{\prime} R\left(t^{\prime}\right)-2 \alpha \pi T \operatorname{sign}(t)
$$

and define

$$
\begin{aligned}
\gamma_{2}^{p_{1}, p_{2}}\left(t, t_{c}\right)= & \frac{d}{d t}\left(\tilde{R}(t) \Theta\left(t-t_{c}\right)\right)+\Theta\left(t-t_{c}\right) \frac{d}{d t} 2 \alpha \pi T \operatorname{sign}(t) \\
& +i p_{2} S(t) \Theta\left(t-t_{c}\right) .
\end{aligned}
$$

With this definition the initial condition in Eq. (3.38) is still fulfilled, since $\tilde{R}(t=0)$ van- 
ishes. Considering only the second term of Eq. (3.115) leads to the differential equations

$$
\begin{aligned}
\frac{d L_{0}}{d t_{c}} & =-i \sum_{p_{1}, p_{2}} 4 \alpha \pi T \delta\left(t_{c}\right) G^{p_{1}}\left(t_{c}\right) G^{p_{2}}(0), \\
\frac{d G^{p}}{d t_{c}} & =-\int_{0}^{t_{c}} d t \sum_{p_{1}, p_{2}} 4 \alpha \pi T \delta\left(t_{c}\right) \\
\times\left(G^{p_{1}}(t) G^{p}(0)-G^{p}(0) G^{p_{1}}(t)\right) G^{p_{2}}\left(t-t_{c}\right), & \\
\frac{d A^{p}}{d t_{c}}(z) & =-\int_{0}^{t_{c}} d t \sum_{p_{1}, p_{2}} 4 \alpha \pi T \delta\left(t_{c}\right) \\
\frac{d B^{p}}{d t_{c}}(z) & =-\int_{0}^{t_{c}} d t \sum_{p_{1}, p_{2}} 4 \alpha \pi T \delta\left(t_{c}\right) \\
\times G^{p_{1}}(t) G^{p}(0) B^{p_{2}}\left(t-t_{c}, z\right), & \\
\frac{d \Sigma}{d t_{c}}(z) & =-\sum_{p_{1}, p_{2}} 4 \alpha \pi T \delta\left(t_{c}\right) A^{p_{1}}\left(t_{c}, z\right) B^{p_{2}}(0, z),
\end{aligned}
$$

where we used $\frac{d}{d t} \operatorname{sign}(\mathrm{t})=2 \delta(\mathrm{t})$. Therefore, this term of $\gamma_{2}^{p_{1}, p_{2}}\left(t, t_{c}\right)$ does not contribute to the renormalization of the vertices $G^{p}, A^{p}(z), B^{p}(z)$. Regarding $L_{0}$ and $\Sigma(z)$ we may account for it by changing the initial conditions:

$$
\begin{aligned}
L_{0}\left(t_{c}=0\right) & =L_{0}-2 i \alpha \pi T M^{2} \\
\Sigma\left(z, t_{c}=0\right) & =-2 \alpha \pi T M^{2} .
\end{aligned}
$$

Let us now consider the remaining terms of Eq. (3.115). Note that for them Eq. (3.39) is fulfilled, since $\lim _{t \rightarrow \infty} \tilde{R}(t)=0$ holds. Using partial integration for the first term, the RG 
equations read

$$
\begin{aligned}
& \frac{d L_{0}}{d t_{c}}=\sum_{p_{1}, p_{2}}\left(\tilde{R}\left(t_{c}\right)\left[G^{p_{1}}\left(t_{c}\right), L_{0}\right]+p_{2} S\left(t_{c}\right) G^{p_{1}}\left(t_{c}\right)\right) G^{p_{2}}(0), \\
& \frac{d G^{p}}{d t_{c}}=\sum_{p_{1}, p_{2}}\left(\tilde{R}\left(t_{c}\right)\left(G^{p_{1}}\left(t_{c}\right) G^{p}(0)-G^{p}(0) G^{p_{1}}\left(t_{c}\right)\right) G^{p_{2}}(0)\right. \\
& -i \int_{0}^{t_{c}} d t\left(G^{p_{1}}(t) G^{p}(0)-G^{p}(0) G^{p_{1}}(t)\right) \\
& \left.\times\left(\tilde{R}\left(t_{c}\right)\left[L_{0}, G^{p_{2}}\left(t-t_{c}\right)\right]+p_{2} S\left(t_{c}\right) G^{p_{2}}\left(t-t_{c}\right)\right)\right), \\
& \frac{d A^{p}}{d t_{c}}(z)=\sum_{p_{1}, p_{2}}\left(\tilde{R}\left(t_{c}\right)\left(A^{p_{1}}\left(t_{c}, z\right) G^{p}(0)-A^{p}(0, z) G^{p_{1}}\left(t_{c}\right)\right) G^{p_{2}}(0)\right. \\
& -i \int_{0}^{t_{c}} d t\left(A^{p_{1}}(t, z) G^{p}(0)-A^{p}(0, z) G^{p_{1}}(t)\right) \\
& \left.\times\left(\tilde{R}\left(t_{c}\right)\left[L_{0}, G^{p_{2}}\left(t-t_{c}\right)\right]+p_{2} S\left(t_{c}\right) G^{p_{2}}\left(t-t_{c}\right)\right)\right), \\
& \frac{d B^{p}}{d t_{c}}(z)=\sum_{p_{1}, p_{2}}\left(\tilde{R}\left(t_{c}\right) G^{p_{1}}\left(t_{c}\right) G^{p}(0) B^{p_{2}}(0, z)\right. \\
& -i \int_{0}^{t_{c}} d t G^{p_{1}}(t) G^{p}(0) \\
& \left.\times\left(\tilde{R}\left(t_{c}\right)\left(L_{0}-z\right)+p_{2} S\left(t_{c}\right)\right) B^{p_{2}}\left(t-t_{c}, z\right)\right), \\
& \frac{d \Sigma}{d t_{c}}(z)=\sum_{p_{1}, p_{2}}-i A^{p_{1}}\left(t_{c}, z\right)\left(\tilde{R}\left(t_{c}\right)\left(L_{0}-z\right)+p_{2} S\left(t_{c}\right)\right) B^{p_{2}}(0, z) .
\end{aligned}
$$

The above equations do not generate the unphysical terms, which are produced by the standard sharp cutoff. We may easily check this by considering again the special case, where $\Delta=\epsilon=T=z=0$. Then, as in case of the sharp cutoff the objects $L_{0}, G^{p}, A^{p}$ and $B^{p}$ commute for all $t_{c}$. This yields

$$
\begin{aligned}
L_{0}\left(t_{c}\right) & =0, \\
G^{p}\left(t_{c}\right) & =G^{p} .
\end{aligned}
$$

Furthermore, this leads to

$$
\begin{aligned}
& A\left(z=0, t_{c}\right)=A^{+}\left(z=0, t_{c}\right)+A^{-}\left(z=0, t_{c}\right)=M, \\
& \Sigma\left(z=0, t_{c}\right)=0,
\end{aligned}
$$


where we again used $d\left(B^{+}-B^{-}\right) / d t_{c}=0$. Finally, one obtains for $B=B^{+}+B^{-}$

$$
\begin{aligned}
B\left(z=0, t_{c}\right) & =M \exp \left(\int_{0}^{t_{c}} d t \tilde{R}(t)\right) \\
& =M \exp \left(-\alpha \ln \left(\frac{1}{1+\left(D t_{c}\right)^{2}}\right)\right) \\
& =M\left(1+\left(D t_{c}\right)^{2}\right)^{\alpha} .
\end{aligned}
$$

Thus, the choice $\gamma_{2}^{p_{1}, p_{2}}\left(t, t_{c}\right)$ does not lead to unphysical divergencies for $D \rightarrow \infty$.

However, the convergence of the RG flow can be improved by switching from $\gamma_{2}^{p_{1}, p_{2}}\left(t, t_{c}\right)$ to $\gamma_{1}^{p_{1}, p_{2}}\left(t, t_{c}\right)$ at some value $t_{c}=\bar{t}[25,26]$. In case of large $\alpha$ this also leads to more accurate results for the equilibrium correlation functions (see Subsection 3.4.4). For $t_{c}>\bar{t} \gg 1 / D, \gamma_{1}^{p_{1}, p_{2}}\left(t, t_{c}\right)$ does not generate any unphysical terms, since then $R(t)$ is approximately independent of $D$. Thus, we define a smooth crossover at $\bar{t}$ with a smearing $\eta$ :

$$
\gamma_{\bar{t} \eta}^{p_{1}, p_{2}}\left(t, t_{c}\right)=\left(1-u_{\eta}\left(t_{c}-\bar{t}\right)\right) \gamma_{2}^{p_{1}, p_{2}}\left(t, t_{c}\right)+u_{\eta}\left(t_{c}-\bar{t}\right) \gamma_{1}^{p_{1}, p_{2}}\left(t, t_{c}\right)
$$

where we use the function

$$
u_{\eta}(x)=\frac{1}{2}+\frac{1}{\pi} \arctan \left(\frac{x}{\eta}\right)
$$

In Eqs. (3.60) - (3.64), we have to substitute

$$
\begin{aligned}
\frac{d}{d t_{c}} \gamma_{\bar{t} \eta}^{p_{1}, p_{2}}\left(t, t_{c}\right)= & \left(1-u_{\eta}\left(t_{c}-\bar{t}\right)\right) \frac{d}{d t_{c}} \gamma_{2}^{p_{1}, p_{2}}\left(t, t_{c}\right)+u_{\eta}\left(t_{c}-\bar{t}\right) \frac{d}{d t_{c}} \gamma_{1}^{p_{1}, p_{2}}\left(t, t_{c}\right) \\
& +\left(\frac{d}{d t_{c}} u_{\eta}\left(t_{c}-\bar{t}\right)\right) \tilde{R}(t)\left(\frac{d}{d t_{c}} \Theta\left(t-t_{c}\right)\right)
\end{aligned}
$$


which leads to the RG equations

$$
\begin{aligned}
& \frac{d L_{0}}{d t_{c}}=\sum_{p_{1}, p_{2}}\left(\left(1-u_{\eta}\left(t_{c}-\bar{t}\right)\right) \tilde{R}\left(t_{c}\right)\left[G^{p_{1}}\left(t_{c}\right), L_{0}\right]\right. \\
& \left.-i U_{\bar{t} \eta}^{p_{2}}\left(t_{c}\right) G^{p_{1}}\left(t_{c}\right)\right) G^{p_{2}}(0), \\
& \frac{d G^{p}}{d t_{c}}=\sum_{p_{1}, p_{2}}\left(\left(1-u_{\eta}\left(t_{c}-\bar{t}\right)\right) \tilde{R}\left(t_{c}\right)\left(G^{p_{1}}\left(t_{c}\right) G^{p}(0)-G^{p}(0) G^{p_{1}}\left(t_{c}\right)\right) G^{p_{2}}(0)\right. \\
& -\int_{0}^{t_{c}} d t\left(G^{p_{1}}(t) G^{p}(0)-G^{p}(0) G^{p_{1}}(t)\right) \\
& \times\left(i\left(1-u_{\eta}\left(t_{c}-\bar{t}\right)\right) \tilde{R}\left(t_{c}\right)\left[L_{0}, G^{p_{2}}\left(t-t_{c}\right)\right]\right. \\
& \left.\left.+U_{\bar{t} \eta}^{p_{2}}\left(t_{c}\right) G^{p_{2}}\left(t-t_{c}\right)\right)\right) \\
& \frac{d A^{p}}{d t_{c}}(z)=\sum_{p_{1}, p_{2}}\left(\left(1-u_{\eta}\left(t_{c}-\bar{t}\right)\right) \tilde{R}\left(t_{c}\right)\left(A^{p_{1}}\left(t_{c}, z\right) G^{p}(0)-A^{p}(0, z) G^{p_{1}}\left(t_{c}\right)\right) G^{p_{2}}(0)\right. \\
& -\int_{0}^{t_{c}} d t\left(\left(A^{p_{1}}(t, z) G^{p}(0)-A^{p}(0, z) G^{p_{1}}(t)\right)\right. \\
& \times\left(i\left(1-u_{\eta}\left(t_{c}-\bar{t}\right)\right) \tilde{R}\left(t_{c}\right)\left[L_{0}, G^{p_{2}}\left(t-t_{c}\right)\right]\right. \\
& \left.\left.+U_{\bar{t} \eta}^{p_{2}}\left(t_{c}\right) G^{p_{2}}\left(t-t_{c}\right)\right)\right) \\
& \frac{d B^{p}}{d t_{c}}(z)=\sum_{p_{1}, p_{2}}\left(\left(1-u_{\eta}\left(t_{c}-\bar{t}\right)\right) \tilde{R}\left(t_{c}\right) G^{p_{1}}\left(t_{c}\right) G^{p}(0) B^{p_{2}}(0, z)\right. \\
& -\int_{0}^{t_{c}} d t G^{p_{1}}(t) G^{p}(0) \\
& \times\left(i\left(1-u_{\eta}\left(t_{c}-\bar{t}\right)\right) \tilde{R}\left(t_{c}\right)\left(L_{0}-z\right)\right. \\
& \left.\left.+U_{\overline{t \eta}}^{p_{2}}\left(t_{c}\right)\right) B^{p_{2}}\left(t-t_{c}, z\right)\right), \\
& \frac{d \Sigma}{d t_{c}}(z)=-\sum_{p_{1}, p_{2}} A^{p_{1}}\left(t_{c}, z\right)\left(i\left(1-u_{\eta}\left(t_{c}-\bar{t}\right)\right) \tilde{R}\left(t_{c}\right)\left(L_{0}-z\right)\right. \\
& \left.+U_{\bar{t} \eta}^{p_{2}}\left(t_{c}\right)\right) B^{p_{2}}(0, z) .
\end{aligned}
$$

Here, we used the function

$$
U_{\bar{t} \eta}^{p_{2}}\left(t_{c}\right)=R\left(t_{c}\right) u_{\eta}\left(t_{c}-\bar{t}\right)+\tilde{R}\left(t_{c}\right) \frac{d}{d t_{c}} u_{\eta}\left(t_{c}-\bar{t}\right)+i p_{2} S\left(t_{c}\right)
$$


where the derivative of $u_{\eta}$ is given by the Lorentzian

$$
\frac{d}{d x} u_{\eta}(x)=\frac{1}{\eta \pi} \frac{\eta^{2}}{x^{2}+\eta^{2}} .
$$

According to Eqs. (3.121) and (3.122) the initial conditions have to be modified again:

$$
\begin{aligned}
L_{0}\left(t_{c}=0\right) & =L_{0}-\left(1-u_{\eta}(-\bar{t})\right) 2 i \alpha \pi T M^{2}, \\
\Sigma\left(z, t_{c}=0\right) & =-\left(1-u_{\eta}(-\bar{t})\right) 2 \alpha \pi T M^{2} .
\end{aligned}
$$

Furthermore, the corresponding RG equations for the calculation of $\Sigma_{D}$ follow from Eqs. (3.87) and (3.88):

$$
\begin{aligned}
& \frac{d D}{d t_{c}}=\sum_{p_{1}, p_{2}}(\left(1-u_{\eta}\left(t_{c}-\bar{t}\right)\right) \tilde{R}\left(t_{c}\right)\left(G^{p_{1}}\left(t_{c}\right) D-D G^{p_{1}}\left(t_{c}\right)\right) G^{p_{2}}(0) \\
&-\int_{0}^{t_{c}} d t\left(G^{p_{1}}(t) D-D G^{p_{1}}(t)\right) \\
& \times\left(i\left(1-u_{\eta}\left(t_{c}-\bar{t}\right)\right) \tilde{R}\left(t_{c}\right)\left[L_{0}, G^{p_{2}}\left(t-t_{c}\right)\right]\right.\left.\left.+U_{\bar{t} \eta}^{p_{2}}\left(t_{c}\right) G^{p_{2}}\left(t-t_{c}\right)\right)\right) \\
& \frac{d \Sigma_{D}}{d t_{c}}(z)=\sum_{p_{1}, p_{2}}\left(\left(1-u_{\eta}\left(t_{c}-\bar{t}\right)\right) \tilde{R}\left(t_{c}\right) A^{p_{1}}\left(t_{c}, z\right) D B^{p_{2}}(0, z=0)\right. \\
&-\int_{0}^{t_{c}} d t A^{p_{1}}(t, z) D\left(i\left(1-u_{\eta}\left(t_{c}-\bar{t}\right)\right) \tilde{R}\left(t_{c}\right) L_{0}\right. \\
&\left.\left.+U_{\bar{t} \eta}^{p_{2}}\left(t_{c}\right)\right) B^{p_{2}}\left(t-t_{c}, z=0\right)\right) .
\end{aligned}
$$

As in case of $G^{p}, A^{p}(z)$ and $B^{p}(z)$, the initial conditions of $D$ and $\Sigma_{D}(z)$ remain unchanged.

The following study of the spin-boson model is based on Eqs. (3.136) - (3.140), (3.144) and (3.145), the final RG equations for the smooth crossover between $\gamma_{2}^{p_{1}, p_{2}}\left(t, t_{c}\right)$ and $\gamma_{1}^{p_{1}, p_{2}}\left(t, t_{c}\right)$. Note that, in the limit of large $\bar{t}$, we again recover Eqs. (3.123) - (3.127), the RG equations for $\gamma_{2}^{p_{1}, p_{2}}\left(t, t_{c}\right)$. This limit is usually applied, when we solve the $\mathrm{RG}$ equations. However, it turns out, that for large couplings $\alpha$ sometimes a crossover around $\bar{t} \approx 1 / \Delta$ yields more stable and/or more accurate results. We then always choose $\eta=0.001 / \Delta$, so that, with $D=100 \Delta$ or $D=1000 \Delta, 1 / D$ is negligible in $\gamma_{1}^{p_{1}, p_{2}}\left(t, t_{c}\right)$, and we do not deal with unphysical terms. 


\subsection{Results}

\subsubsection{The renormalized tunnel matrix element}

Because of its nonperturbative character the real-time renormalization group is able to determine the renormalized tunnel matrix element $\Delta_{r}$ of the spin-boson model. As already mentioned in Subsection 3.2.3, the flow equations for the Hamiltonian $H_{0}$ of the two-state system is obtained from the RG equations on the Keldysh contour by setting $p=p_{1}=p_{2}=+$ and replacing $L_{0}$ by $H_{0}$ as well as $G^{+}$by $\sigma_{z} / 2$. Furthermore, we expand the exponentials in $t_{c}$. Analogously to the Poor Man's scaling approach (see Section 3.1), $\Delta_{r}$ at $T=0$ is given by the crossover energy scale defined by $\Delta_{r}=\Delta\left(\tilde{t}_{c}\right)=1 / \tilde{t}_{c}$.

In lowest order in $t_{c}$ we obtain for $\Delta\left(t_{c}\right)$ from Eq. (3.136)

$$
-\frac{1}{2} \frac{d \Delta}{d t_{c}}\left(t_{c}\right)=\left(1-u_{\eta}\left(t_{c}-\bar{t}\right)\right) \tilde{R}\left(t_{c}\right) \frac{\Delta\left(t_{c}\right)}{4}-U_{\bar{t} \eta}\left(t_{c}\right) t_{c} \frac{\Delta\left(t_{c}\right)}{4}+\mathcal{O}\left(\alpha^{2}\right) .
$$

We now consider the limit of $\bar{t} \rightarrow \infty$, so that we may set $u_{\eta}\left(t_{c}-\bar{t}\right)=d / d t_{c} u_{\eta}\left(t_{c}-\bar{t}\right)=0$. The resulting flow equation then reads

$$
\frac{d \Delta}{d t_{c}}\left(t_{c}\right)=\frac{\Delta\left(t_{c}\right)}{2}\left(-\tilde{R}\left(t_{c}\right)+i S\left(t_{c}\right) t_{c}\right)+\mathcal{O}\left(\alpha^{2}\right) .
$$

In the following we neglect the terms of the order $\alpha^{2}$. To determine $\Delta_{r}$ we take the real part of $\Delta\left(t_{c}\right)$. Performing the integral yields

$$
\ln \left(\frac{\Delta_{r}}{\Delta}\right)=-\frac{\alpha}{2} \ln \left(1+\left(\frac{D}{\Delta_{r}}\right)^{2}\right) .
$$

Considering the above equation in the scaling limit, where $D \gg \Delta_{r}$ holds, leads to

$$
\frac{\Delta_{r}}{\Delta}=\left(\frac{\Delta}{D}\right)^{\frac{\alpha}{1-\alpha}}
$$

which coincides with the well-known result in Eq. (3.11). Thus our approach contains the correct relevant energy scale. Also note that for the spin-boson model the renormalizationgroup flow of the couplings yields $\alpha \rightarrow 0$, if for the bare coupling $\alpha<1$ holds (see Fig. 3.2). Therefore, Eqs. (3.136) - (3.140), (3.144) and (3.145) are able to describe the spin-boson model accurately in a nonperturbative way.

In the following we present numerical results obtained from these equations both for the reduced density matrix and for equilibrium correlation functions. The correspondence of the expectation values of the Pauli matrices to the matrix elements of the reduced density matrix $p(t)$ reads

$$
\begin{aligned}
\left\langle\sigma_{x}(t)\right\rangle & =p_{u d}(t)+p_{d u}(t)=2 \operatorname{Re}\left[p_{u d}(t)\right] \\
\left\langle\sigma_{y}(t)\right\rangle & =i\left(p_{u d}(t)-p_{d u}(t)\right)=-2 \operatorname{Im}\left[p_{u d}(t)\right], \\
\left\langle\sigma_{z}(t)\right\rangle & =p_{u u}(t)-p_{d d}(t)=2 p_{u u}(t)-1
\end{aligned}
$$


The second equations in Eq. (3.150) follow from the Hermiticity of $p(t)$ and the normalization $p_{u u}+p_{d d}=1$. Furthermore, $\left\langle\sigma_{y}\right\rangle$ and $\left\langle\sigma_{z}\right\rangle$ are connected via the Heisenberg equation which yields

$$
\left\langle\dot{\sigma}_{z}\right\rangle=-\Delta\left\langle\sigma_{y}\right\rangle .
$$

\subsubsection{Static properties}

The static properties of the spin-boson model are determined by Eq. (3.34). We calculate the kernel $\Sigma(z=0)$ by integrating the ordinary differential equations Eqs. (3.136) (3.140) using the method described in Appendix E.1. As we have already mentioned, the time-integrals on the right-hand sides of the RG equations only refer to the interaction picture. Thus they can be performed analytically. For the evaluation of the exponentials of $L_{0}$ we introduce a unitary transformation which diagonalizes $L_{0}$, see Appendix E. 2 for details. For the calculation of the stationary reduced density matrix $p_{s t}$ we consider the limit of $\bar{t} \rightarrow \infty$ in Eq. (3.133), i.e. we use the definition of $\gamma_{2}^{p_{1}, p_{2}}\left(t, t_{c}\right)$. Figs. 3.7 and 3.8 show the real part of the matrix elements of $p_{s t}$ as a function of $t_{c}$ for different values of $\epsilon$ and $\alpha$ with $D=100 \Delta$ and $T=0$. Note that $p_{s t}$ converges for $t_{c} \rightarrow \infty$. Whereas for finite $t_{c}$ the stationary reduced density matrix has unphysical eigenvalues (see our remarks in Subsection 3.2.3), its asymptotic value corresponds to a well-defined reduced density matrix. The imaginary part of $p_{s t}$ vanishes for all $t_{c}$ which is consistent with Eq. (3.151). For the symmetric case the diagonal elements of the real part are also constant $(=1 / 2)$. In case of $\epsilon \neq 0$ the diagonal elements are renormalized, and the asymmetry leads to a finite difference between them for $t_{c} \rightarrow \infty$. The off-diagonal elements of the real part of $p_{s t}$ are renormalized in any case and attain a finite value for $t_{c} \rightarrow \infty$.

We may now calculate the static susceptibility according to Eq. (3.35) from the asymptotic $\left(t_{c} \rightarrow \infty\right)$ results for $p_{s t}$. The derivative with respect to $\epsilon$ is performed by calculating $p_{s t}$ for an increment $d \epsilon$. We set $d \epsilon=0.001 \Delta$, which turns out to be sufficiently small. The temperature-dependence of the static susceptibility $\chi_{0}(T)$ for different values of $D, \alpha$ and $\epsilon$ is shown in Figs. 3.9 - 3.14. In Fig. 3.9 one sees the susceptibility for $D=100 \Delta$ and $D=1000 \Delta$ with $\alpha=0.1$ and $\epsilon=0$, where we again chose $\bar{t} \rightarrow \infty$. Using Eq. (3.149) this yields renormalized tunnel matrix elements of $\Delta_{r}=0.599 \Delta$ and $\Delta_{r}=0.464 \Delta$. Fig. 3.9 shows that $\chi_{0}(T)$ strongly depends on the high-energy cutoff $D$. However, in the scaling limit the susceptibility should only depend on one energy scale. We normalize our results using the zero-temperature value $\chi_{0}(T=0)$. Then the curves for different high-energy cutoffs coincide. The normalized results for different values of $\alpha$ and $\epsilon$ can be seen in Figs. 3.10 and 3.11. Whereas for $\epsilon=0$ the susceptibility is a monotonous function of $T$, we obtain a local maximum for the biased case. In Fig. 3.12 our result for $\alpha=0.125$ and $\epsilon=0$ is compared to that obtained for the anisotropic Kondo model using the Bethe ansatz. One recognizes a good agreement, which can be improved by choosing a finite value of the crossover parameter $\bar{t} \approx 1 / \Delta$ (as mentioned in Section 3.3 we set $\eta=0.001 / \Delta$ for the smearing). For larger $\alpha$ this finite value of $\bar{t}$ also avoids numerical instabilities, and we will later see that the errors concerning the Shiba-relation can be reduced for $\alpha \approx 0.2$ by setting $\bar{t} \approx 1 / \Delta$. For the biased case the results of the Bethe 


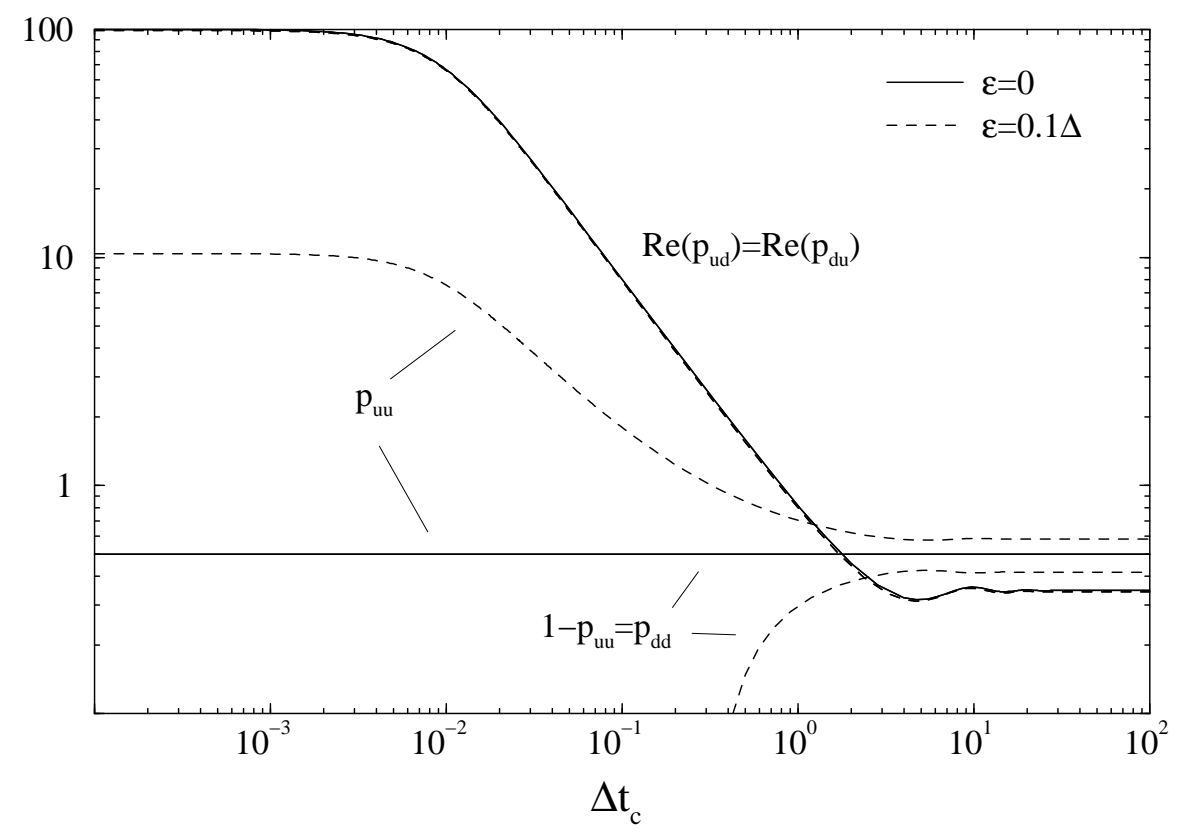

Figure 3.7: Real part of the stationary reduced density matrix $p_{s t}$ as a function of $t_{c}$ for $\alpha=0.1, D=100 \Delta$ and $T=0$. Solid lines: $\epsilon=0$. Dashed lines: $\epsilon=0.1 \Delta$.

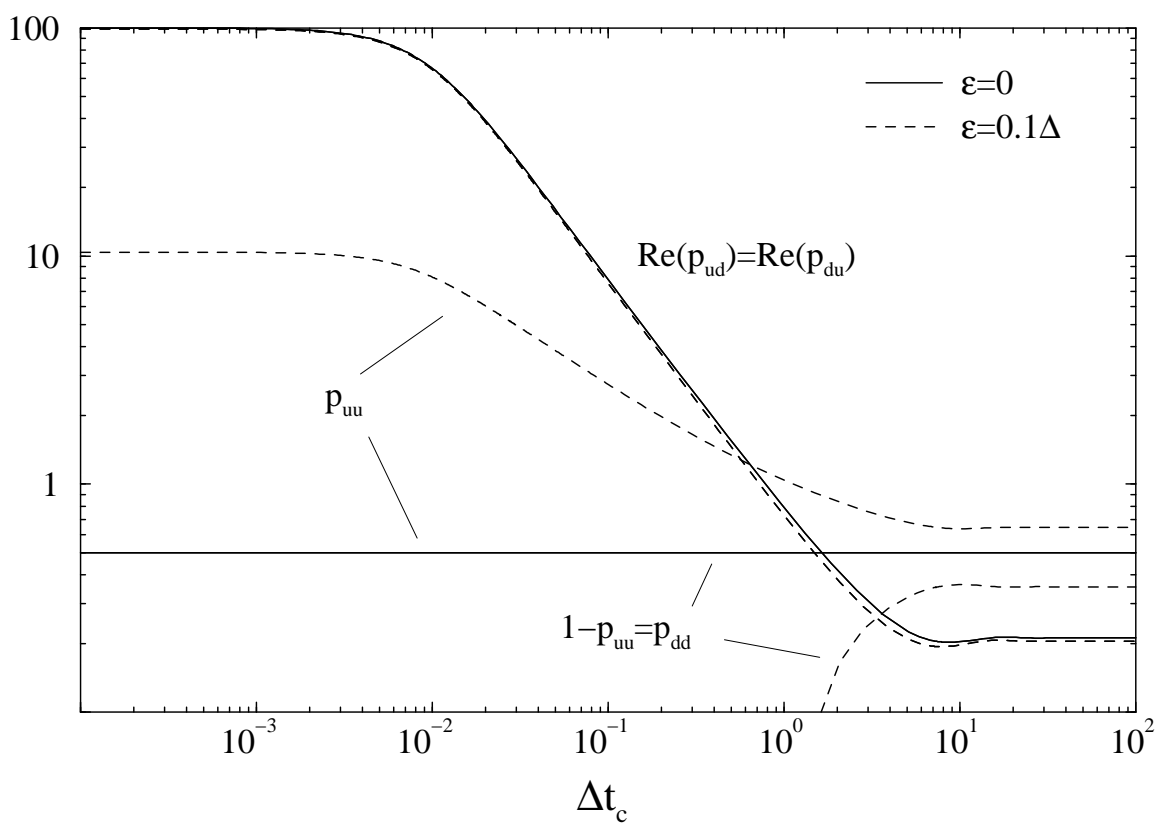

Figure 3.8: Real part of the stationary reduced density matrix $p_{s t}$ as a function of $t_{c}$ for $\alpha=0.2, D=100 \Delta$ and $T=0$. Solid lines: $\epsilon=0$. Dashed lines: $\epsilon=0.1 \Delta$. 


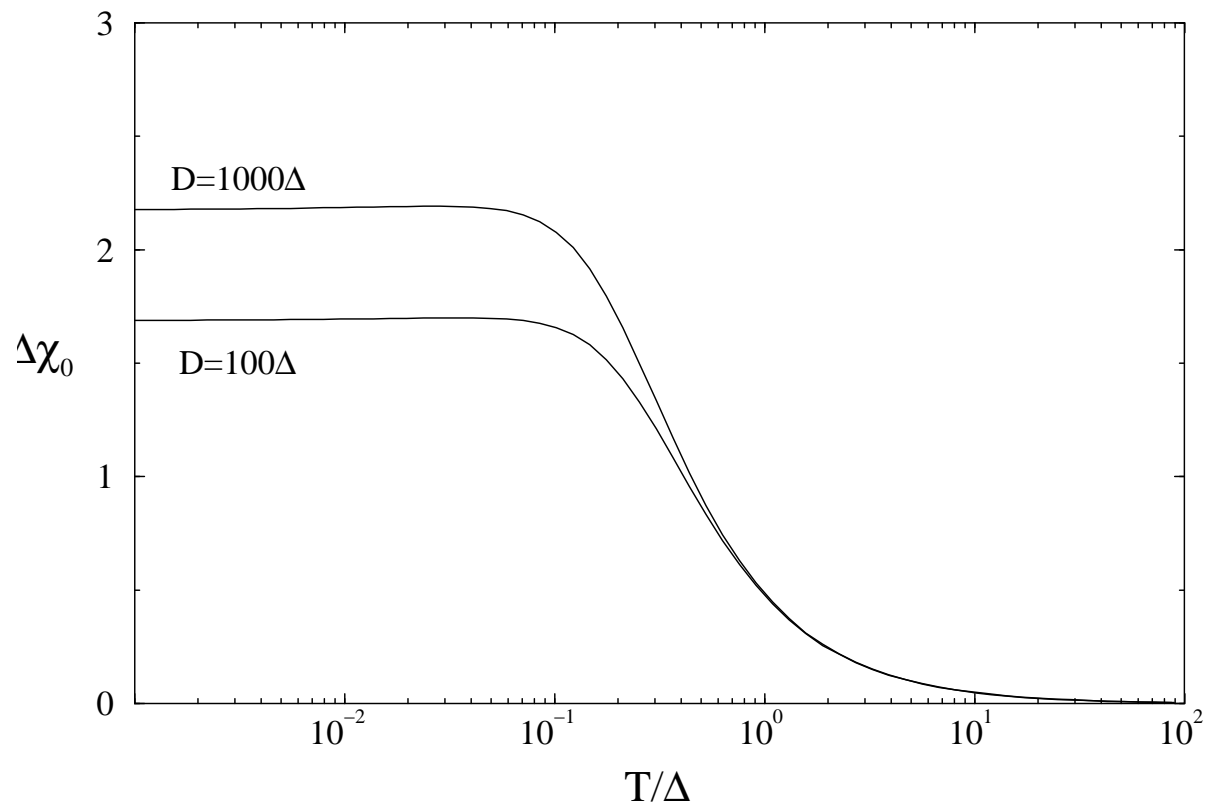

Figure 3.9: Static susceptibility $\chi_{0}$ as a function of the temperature $T$ for $\alpha=0.1, \epsilon=0$ and $D=100 \Delta, 1000 \Delta$.

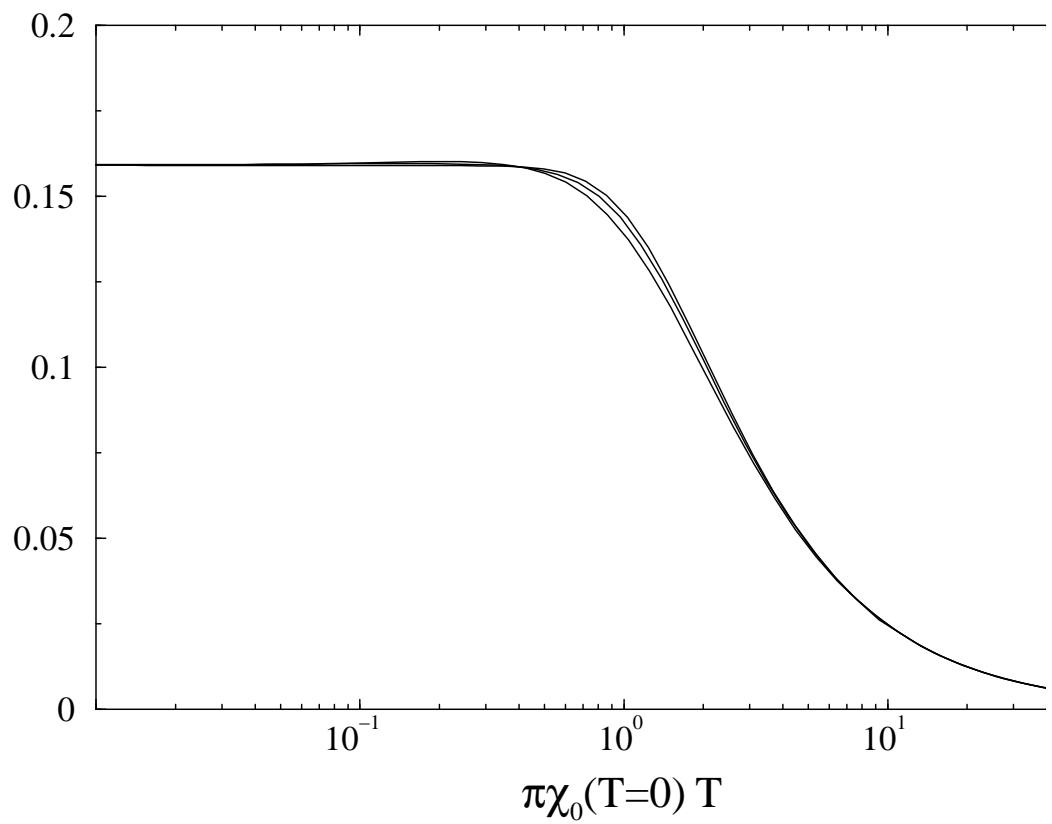

Figure 3.10: Static susceptibility $\chi_{0}$ as a function of the temperature $T$ for $\epsilon=0$, $D / \Delta=1000 \gg \Delta_{r} / \Delta$ and $\alpha=0.01,0.05,0.125$ (from top to bottom). 


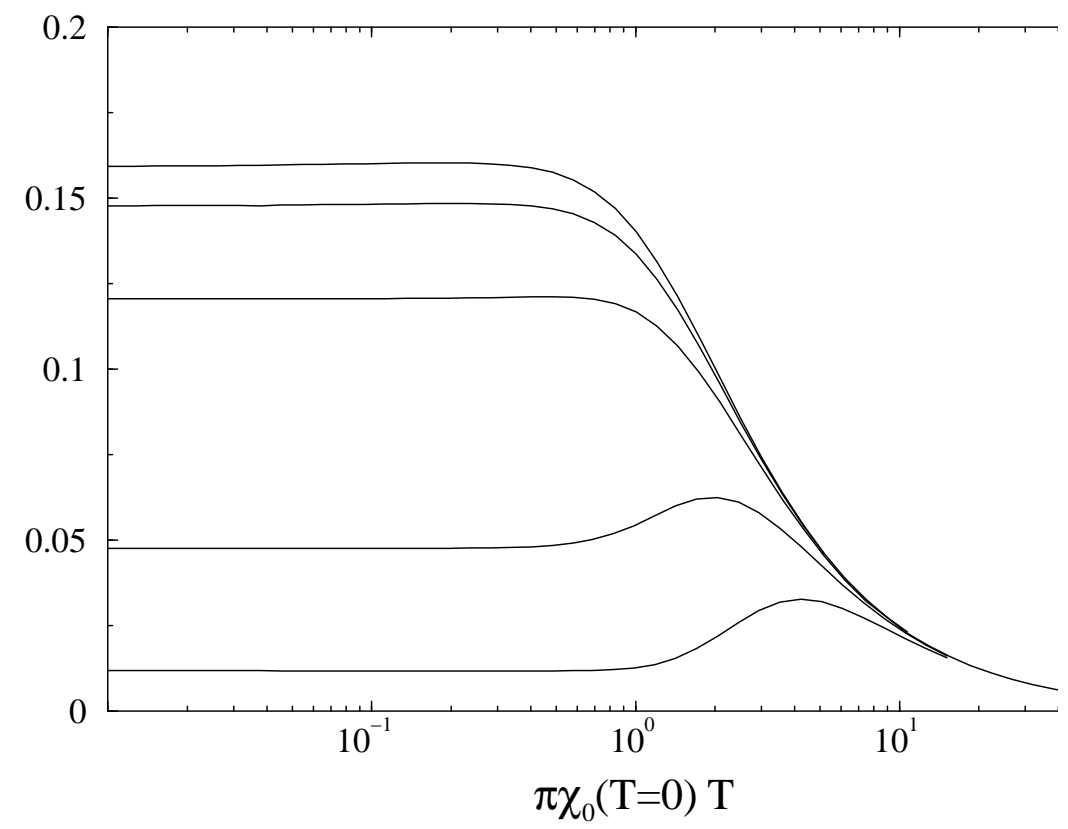

Figure 3.11: Static susceptibility $\chi_{0}$ as a function of the temperature $T$ for $\alpha=0.1$, $D / \Delta=1000 \gg \Delta_{r} / \Delta$ and $\epsilon / \Delta=0,0.1,0.2,0.5,1$ (from top to bottom).

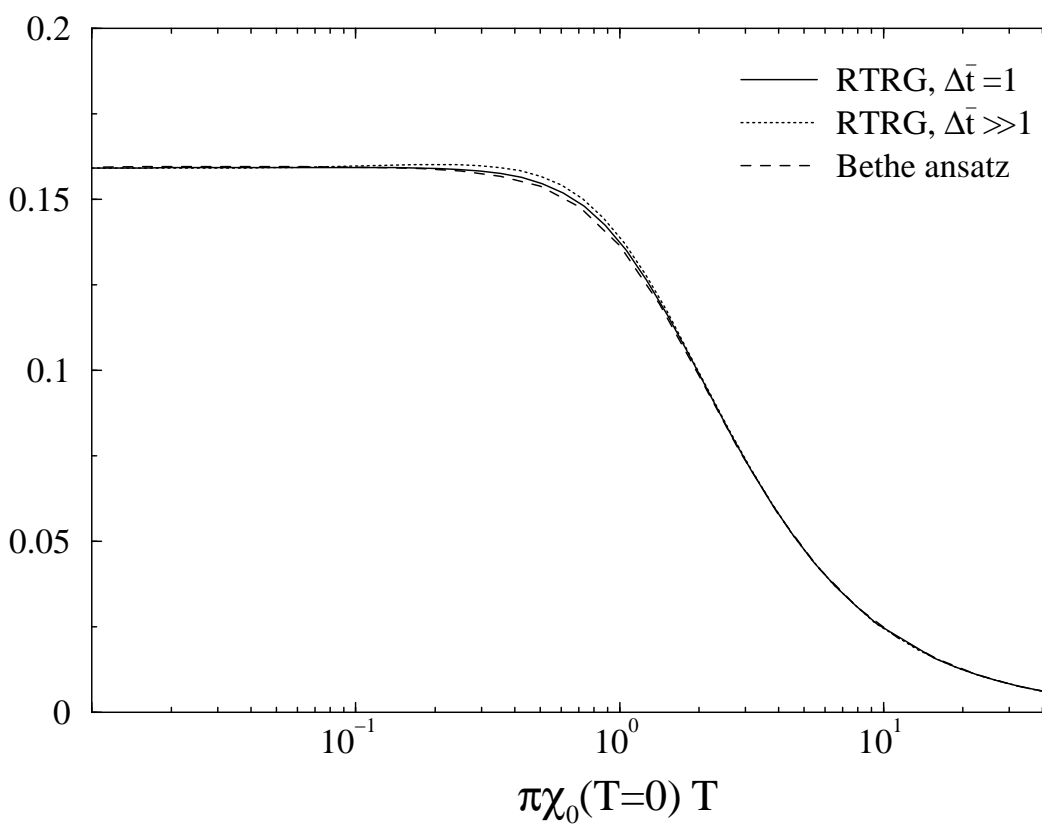

Figure 3.12: Static susceptibility $\chi_{0}$ as a function of the temperature $T$ for $\alpha=0.125$, $\epsilon=0$ and $D / \Delta=1000 \gg \Delta_{r} / \Delta$. Solid line: RTRG with $\bar{t}=1 / \Delta$. Dotted line: RTRG with $\bar{t} \rightarrow \infty$. Dashed line: Bethe ansatz for the anisotropic Kondo model. 


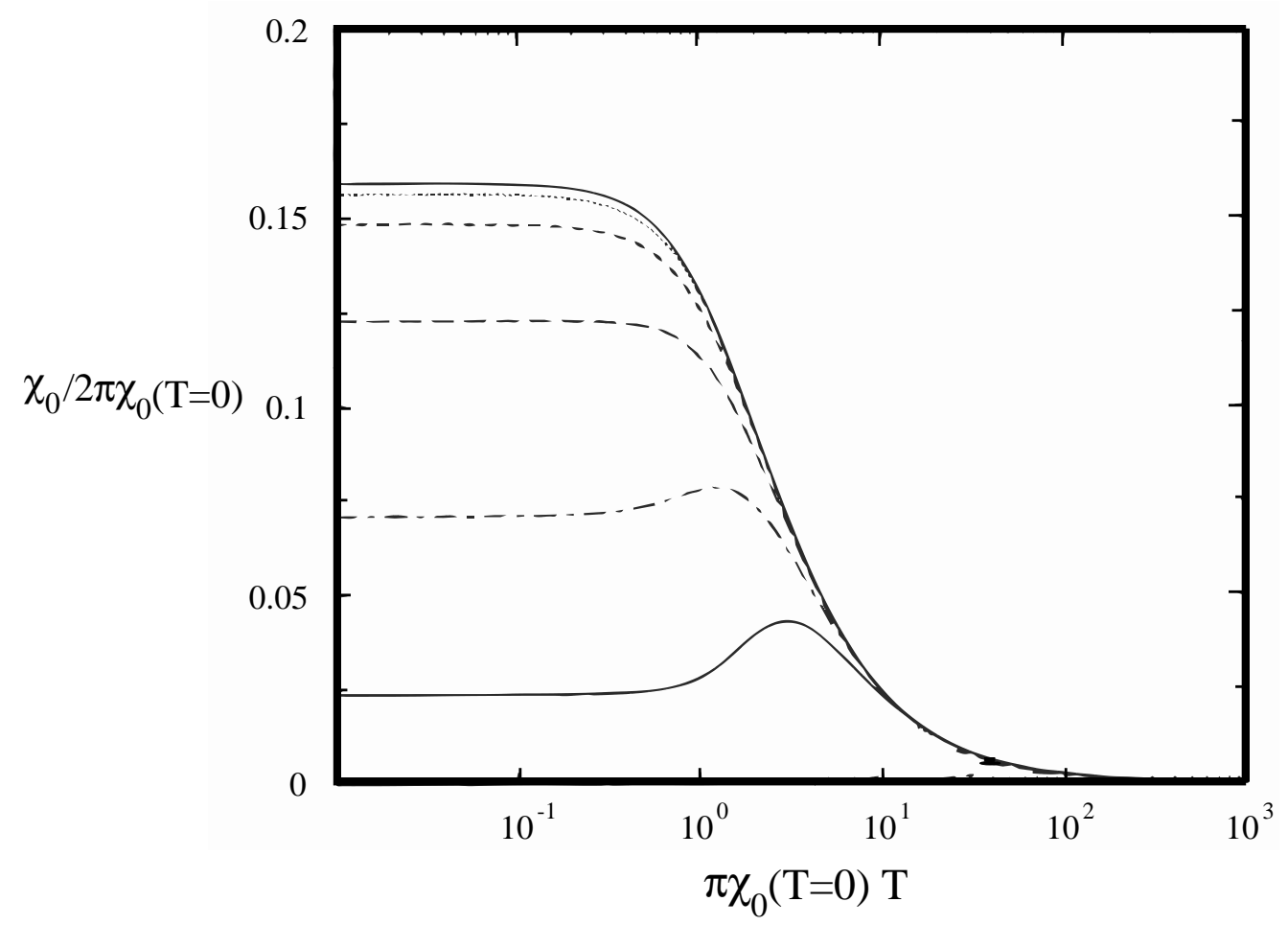

Figure 3.13: Static susceptibility $\chi_{0}$ as a function of the temperature $T$ for $\alpha=0.2, D \gg$ $\Delta_{r}$ and $\pi \chi_{0}(T=0) \epsilon=0,0.3125,0.625,1.25,2.5,5$ (from top to bottom), obtained by the Bethe ansatz for the anisotropic Kondo model (taken from Ref. [28]).

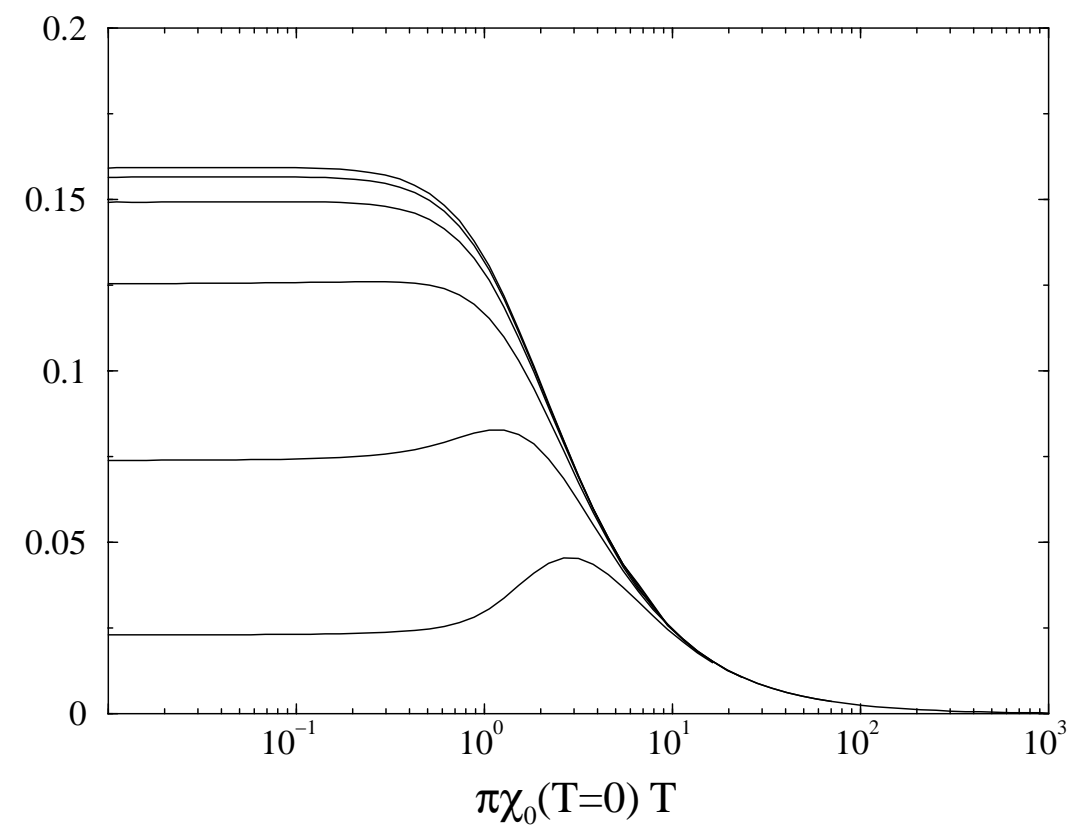

Figure 3.14: Static susceptibility $\chi_{0}$ as a function of the temperature $T$ for $\alpha=0.2$, $D / \Delta=1000 \gg \Delta_{r} / \Delta, \bar{t}=1 / \Delta$ and $\pi \chi_{0}(T=0) \epsilon=0,0.3125,0.625,1.25,2.5,5$ (from top to bottom). 
ansatz for $\alpha=0.2$ are shown in Fig. 3.13 [28]. Our corresponding results are presented in Fig. 3.14, where we have used $\bar{t}=1 / \Delta$. Comparing them one recognizes deviations at low temperature for $\epsilon \neq 0$. These deviations occur independently of the choice of $\bar{t}$, which demonstrates that the commonly used relation of the parameters (see Appendix B) of the Kondo model to the ones of the spin-boson model has to be taken with care.

Finally, we note that in the high-temperature limit all our results for $\chi_{0}(T)$ show the correct $1 / 2 T$ law, which is independent of $\alpha, \epsilon$ and $D$ [28].

\subsubsection{Dynamics}

In this subsection we calculate the time-dependent reduced density matrix $p(t)$ for the initial preparation of the two-state system in the spin-up state: $p(0)_{u u}=1$, $p(0)_{d d}=p(0)_{u d}=p(0)_{d u}=0$. From the result for $\Sigma(z)$ for $t_{c} \rightarrow \infty$ and from Eq. (3.32) we determine the Laplace transform $p(z)$. According to Eq. (3.31) $p(t)$ is then given by

$$
\begin{aligned}
p(t) & =\frac{1}{2 \pi} \int_{-\infty+i c}^{\infty+i c} d z e^{-i z t} p(z) \\
& =\frac{1}{2 \pi} e^{c t} \int_{-\infty}^{\infty} d z e^{-i z t} p(z+i c) \quad \text { for } \quad c \in \mathbb{R}^{+}
\end{aligned}
$$

This Fourier integral is calculated numerically using the method described in Appendix E.3 and setting $c=0.1 \Delta$.

The solution for the time evolution of the reduced density matrix at vanishing temperature is shown in Figs. 3.15 - 3.20 for different couplings and for the unbiased and biased case. Here, we again chose $\bar{t} \rightarrow \infty$, in fact these results are insensitive to the choice of $\bar{t}$ even for a coupling strength of $\alpha=0.2$. Figs. 3.15 and 3.16 show, that, for $\alpha=0.1$, $\epsilon=0$ and $D=100 \Delta$ we achieve a very good agreement with chromostochastic quantum dynamics (CSQD). Only the real parts of the nondiagonal elements, which correspond to $\left\langle\sigma_{x}\right\rangle$, exhibit a deviation of approximately $5 \%$. However, in contrast to the results for $\left\langle\sigma_{z}\right\rangle$ CSQD cannot give an error for $\left\langle\sigma_{x}\right\rangle$ [74]. The diagonal elements oscillate in time as well as the imaginary parts of the off-diagonal elements, in fact the latter do not contain any new information due to Eq. (3.151), so that we focus on the real part of $p(t)$ in the following. For the symmetric case the real parts of the off-diagonal elements show a pure decaying behaviour (Fig. 3.15). In Fig. 3.17 the correct scaling behaviour is checked for $D=100 \Delta$ respectively $D=1000 \Delta$ by rescaling the results using $\Delta_{r}=0.599 \Delta$ respectively $\Delta_{r}=0.464 \Delta$. One obtains coincidence of the diagonal elements, whereas the real parts of the off-diagonal elements have an extra factor of $\Delta_{r} / \Delta$, which is consistent with Ref. [1]. A comparison with the noninteracting blip approximation (NIBA) both for the unbiased and the biased case is drawn in Figs. 3.18 and 3.19. Note that a formal derivation of the NIBA results is presented in Appendix A. In Figs. 3.18 and 3.19, $p(t)$ is calculated from Eqs. (A.14) - (A.19), where we again used the technique presented in Appendix E.3. One recognizes that for $\epsilon=0$ the NIBA gives quite accurate results for the diagonal elements (see also Appendix A), but fails for the nondiagonal elements. The 


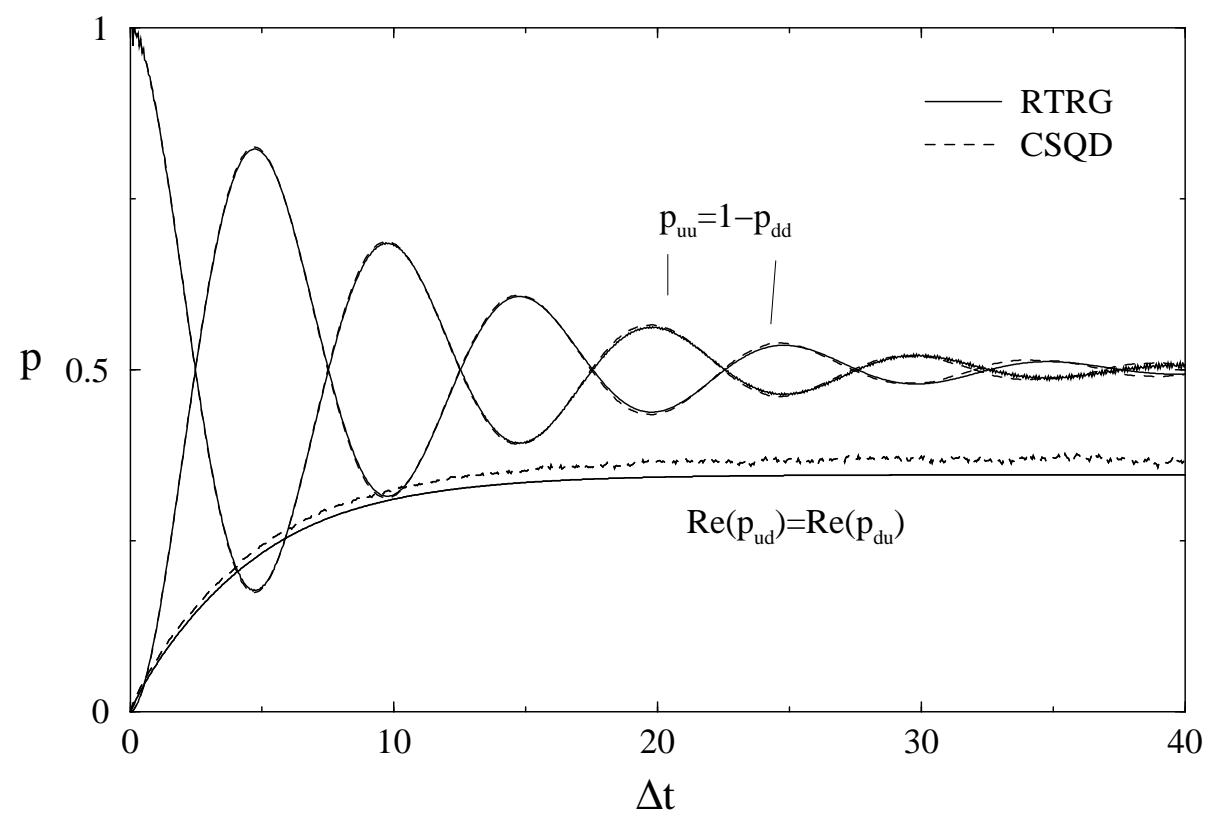

Figure 3.15: Real part of the time-dependent reduced density matrix $p(t)$ for $\alpha=0.1$, $\epsilon=0, D=100 \Delta$ and $T=0$. Solid lines: RTRG. Dashed lines: CSQD.

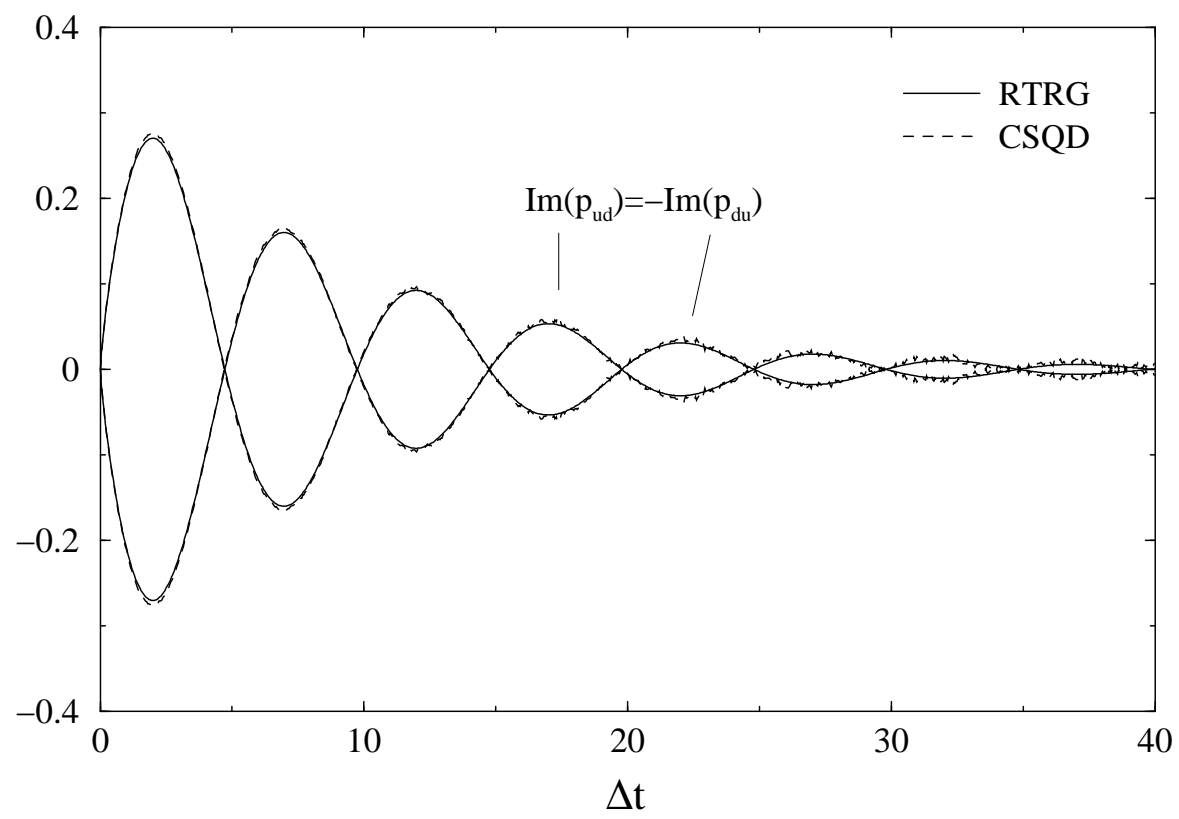

Figure 3.16: Imaginary part of the time-dependent reduced density matrix $p(t)$ for $\alpha=0.1, \epsilon=0, D=100 \Delta$ and $T=0$. Solid lines: RTRG. Dashed lines: CSQD. 


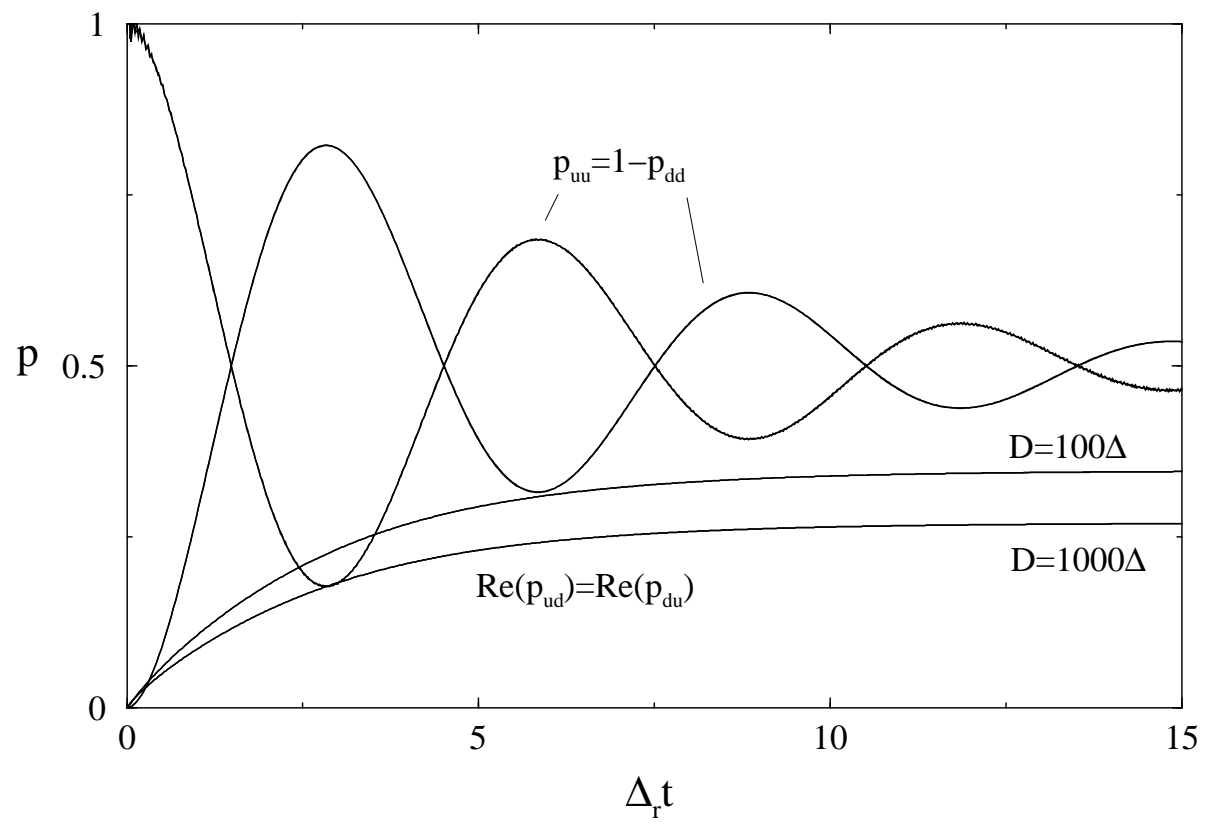

Figure 3.17: Rescaled real part of the time-dependent reduced density matrix $p(t)$ for $\alpha=0.1, \epsilon=0, T=0$ and $D=100 \Delta, 1000 \Delta$. The nondiagonal elements have an extra factor $\Delta_{r} / \Delta=0.599 \Delta, 0.464 \Delta$.

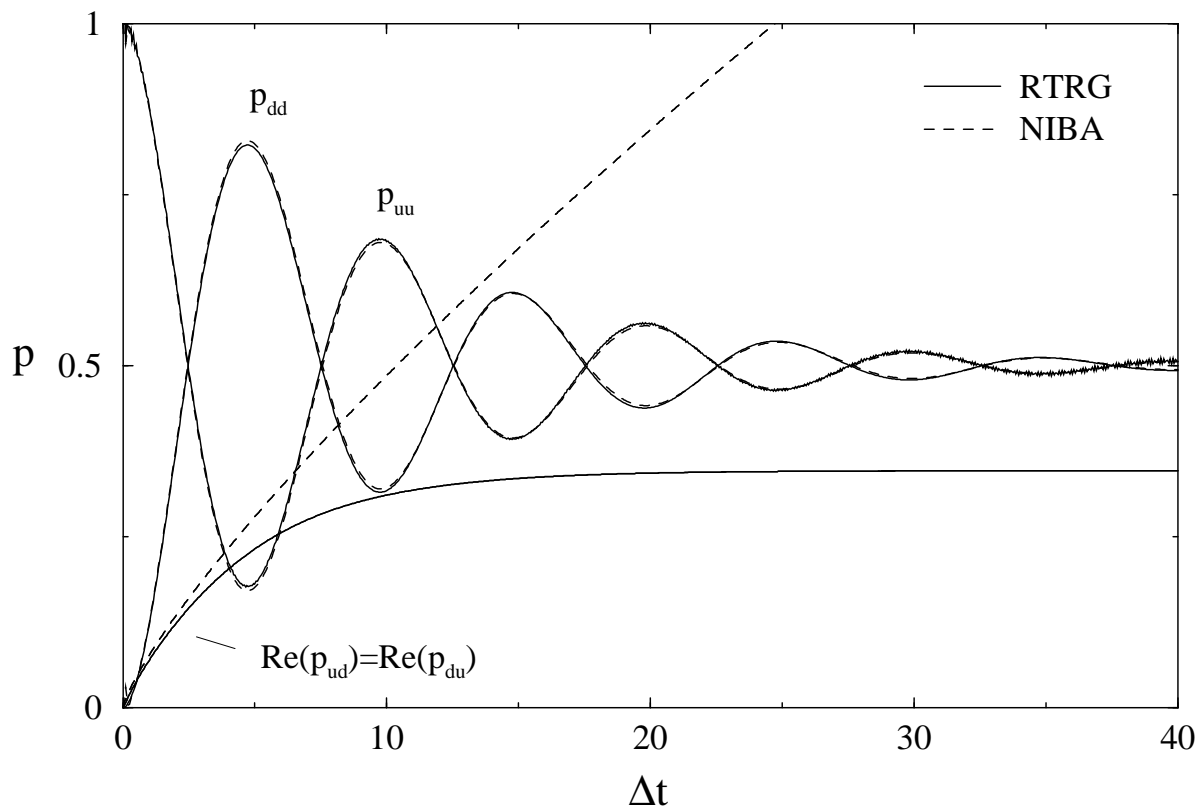

Figure 3.18: Real part of the time-dependent reduced density matrix $p(t)$ for $\alpha=0.1$, $\epsilon=0, D=100 \Delta$ and $T=0$. Solid lines: RTRG. Dashed lines: NIBA. 


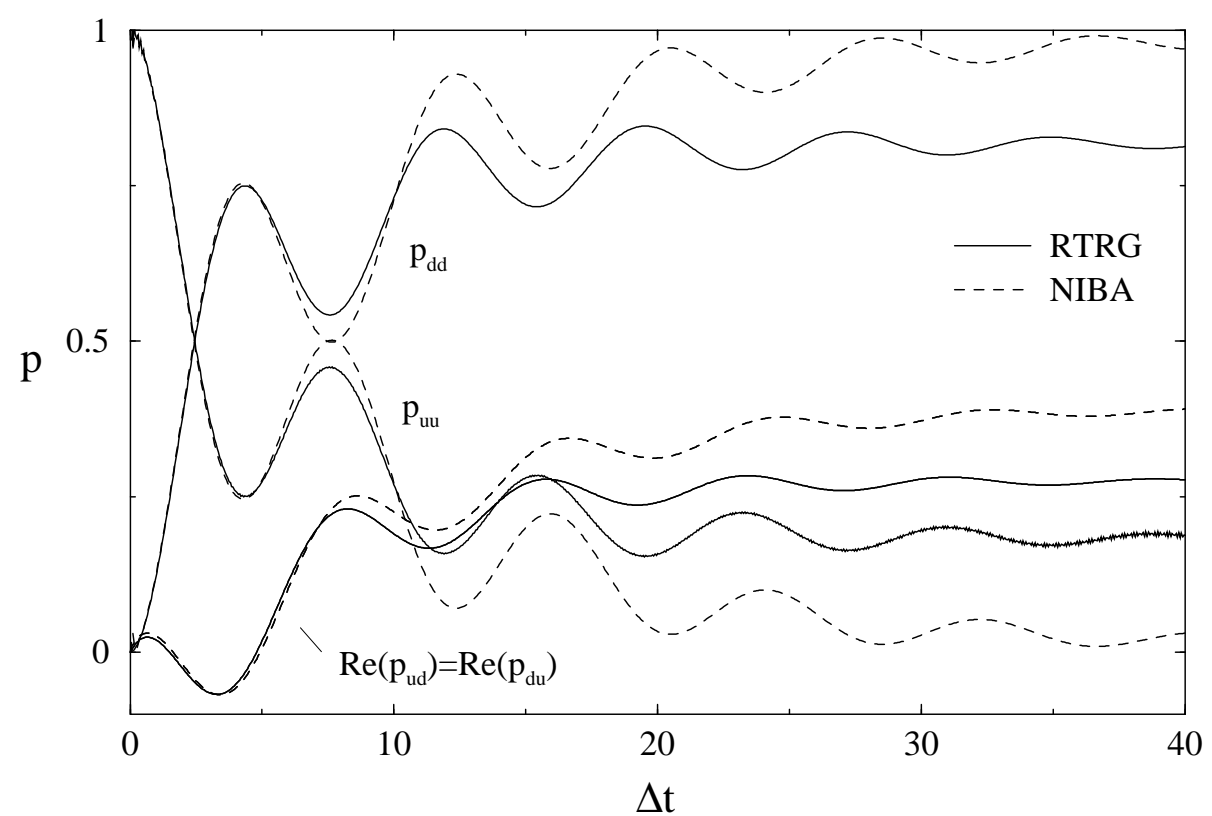

Figure 3.19: Real part of the time-dependent reduced density matrix $p(t)$ for $\alpha=0.1$, $\epsilon=0.5 \Delta, D=100 \Delta$ and $T=0$. Solid lines: RTRG. Dashed lines: NIBA.

NIBA even violates the bound

$$
\left\langle\sigma_{x}(t)\right\rangle^{2} \leq 1
$$

which follows from $\left\langle\sigma_{x}^{2}(t)\right\rangle-\left\langle\sigma_{x}(t)\right\rangle^{2} \geq 0$. For finite bias (see Fig. 3.19) the diagonal elements also have a decaying part, as well as the nondiagonal elements also show oscillations. We see, that, for $\epsilon=0.5 \Delta$ the NIBA gives poor results both for the diagonal and the nondiagonal elements. In Fig. 3.20 our results for the unbiased and the biased case are shown for $\alpha=0.2$.

For one of these plots of $p(t)$ we calculated 512 points of the Laplace transform $p(z+i c)$ for $z \in[-10 \Delta, 10 \Delta]$. For $z<-10 \Delta$ respectively $z>10 \Delta$ we extrapolated $p(z+i c)$ algebraically (see also Appendix E.3). For such an integration problem a parallelization of the computer program may strongly improve the performance. We applied the "pvm" package to parallelize our program [75]. Using a cluster of four PIII (500MHz)-machines, one of these plots of $p(t)$ is generated within about five hours only.

From the above results for $p(t)$ we also determine the oscillation frequency $\Omega \sim \Delta_{r}$ of the diagonal elements. We compare it with the analytical result $\Omega_{0}$, which is valid in the limit of small $\alpha$ only. It reads [1]

$$
\Omega_{0}=\left(\epsilon^{2}+(\Gamma(1-2 \alpha) \cos (\pi \alpha))^{1 / 1-\alpha} \Delta_{r}^{2}\right)^{1 / 2}
$$

Furthermore, for the symmetric case, conformal field theory (CFT) applied to the anisotropic 


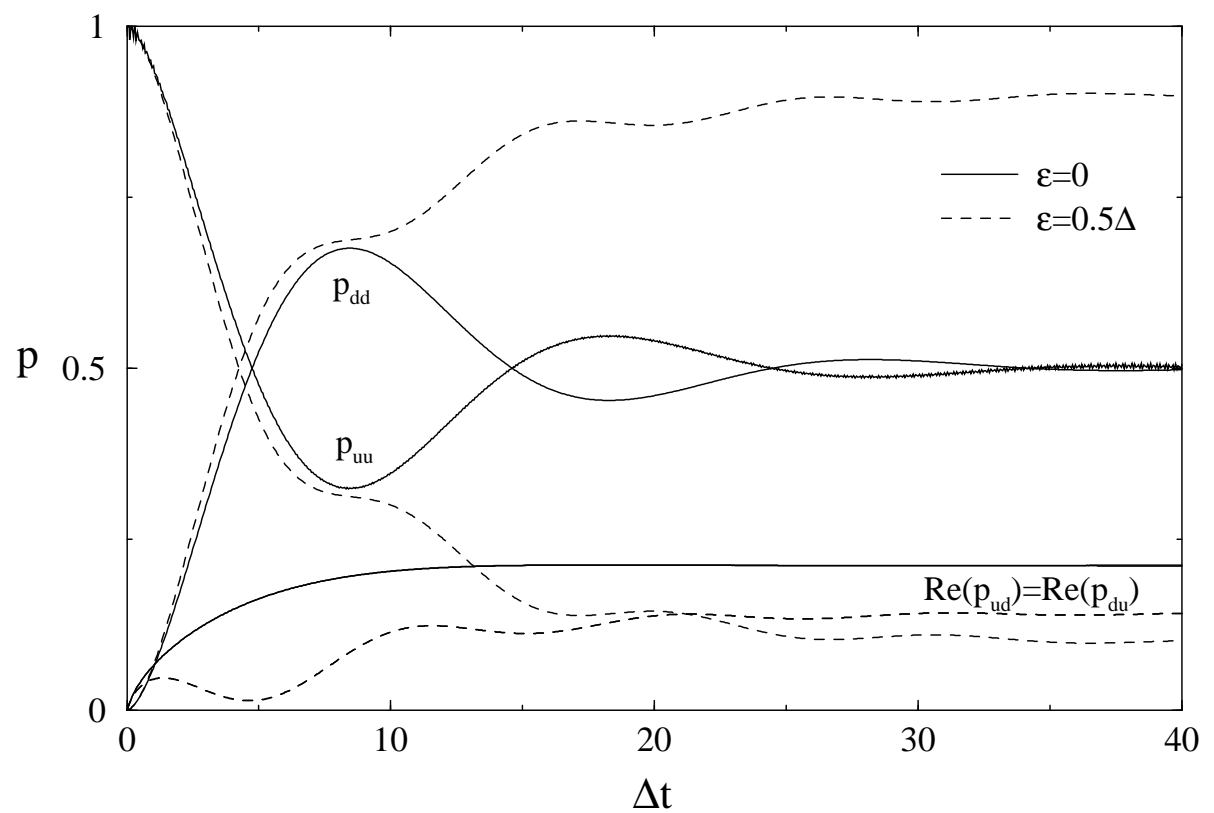

Figure 3.20: Real part of the time-dependent reduced density matrix $p(t)$ for $\alpha=0.2$, $D=100 \Delta$ and $T=0$. Solid lines: $\epsilon=0$. Dashed lines: $\epsilon=0.5 \Delta$.

Kondo model, leads to the solution [29, 1]

$$
\begin{aligned}
\Omega_{C F T}= & \sin (\pi \alpha / 2(1-\alpha)) \cos (\pi \alpha / 2(1-\alpha)) \frac{\Gamma(\alpha / 2(1-\alpha))}{\sqrt{\pi} \Gamma(1 / 2(1-\alpha))} \\
& \times\left(\frac{\Gamma\left(\frac{1}{2}+\alpha\right) \Gamma(1-\alpha) \Gamma(1-2 \alpha) \cos (\pi \alpha)}{\sqrt{\pi}}\right)^{1 / 2(1-\alpha)} \Delta_{r},
\end{aligned}
$$

which is valid for $0<\alpha<0.5$. Figs. 3.21 and 3.22 show $\Omega$ as a function of the coupling strength $\alpha$ for the unbiased and the biased case with $D=100 \Delta$ and $T=0$. As expected, in the limit of small $\alpha$ our results are consistent with $\Omega_{0}$, while for large $\alpha$ there are deviations. In comparison to the CFT result for the symmetric case we find a good agreement, only for large $\alpha$ there are small deviations.

The long-time behaviour of $p(t)$ can be approximated by an exponential decay, which is parametrized by the dephasing time $\tau^{d e p h}$ and the relaxation time $\tau^{r e l}$ :

$$
p(t)=p_{0}+p_{1} e^{i \Omega t} e^{-t / \tau^{d e p h}}+p_{2} e^{-t / \tau^{r e l}} .
$$

The results for $\alpha \ll 1 \operatorname{read}[1]$

$$
\begin{aligned}
\tau_{0}^{\text {deph }} & =\frac{2(\Gamma(1-2 \alpha) \cos (\pi \alpha))^{1 /(\alpha-1)} \Omega_{0}}{\pi \alpha \Delta_{r}^{2}}, \\
\tau_{0}^{r e l} & =\frac{(\Gamma(1-2 \alpha) \cos (\pi \alpha))^{1 /(\alpha-1)} \Omega_{0}}{\pi \alpha \Delta_{r}^{2}} .
\end{aligned}
$$




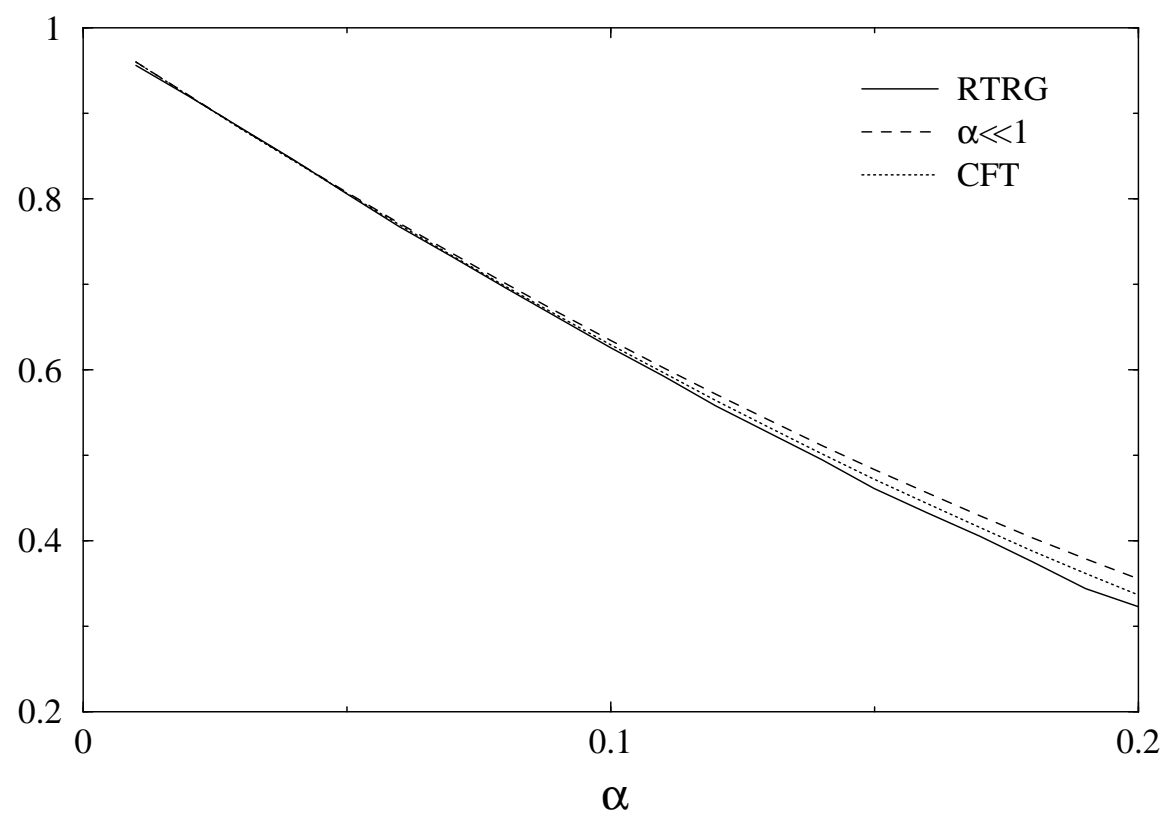

Figure 3.21: Oscillation frequency $\Omega$ as a function of the coupling constant $\alpha$ for $\epsilon=0$, $D=100 \Delta$ and $T=0$. Solid line: RTRG. Dashed line: $\Omega_{0}(\alpha \ll 1)$. Dotted line: $\Omega_{C F T}$.

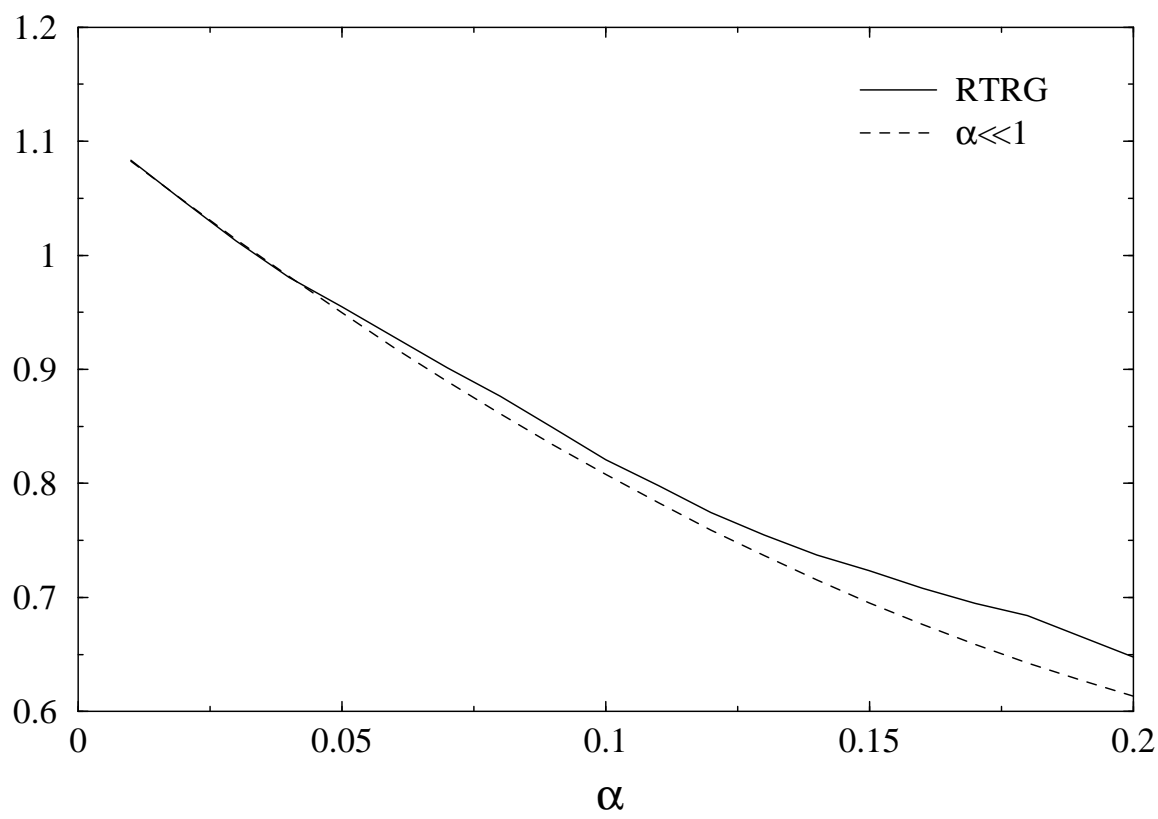

Figure 3.22: Oscillation frequency $\Omega$ as a function of the coupling constant $\alpha$ for $\epsilon=0.5 \Delta, D=100 \Delta$ and $T=0$. Solid line: RTRG. Dashed line: $\Omega_{0}(\alpha \ll 1)$. 


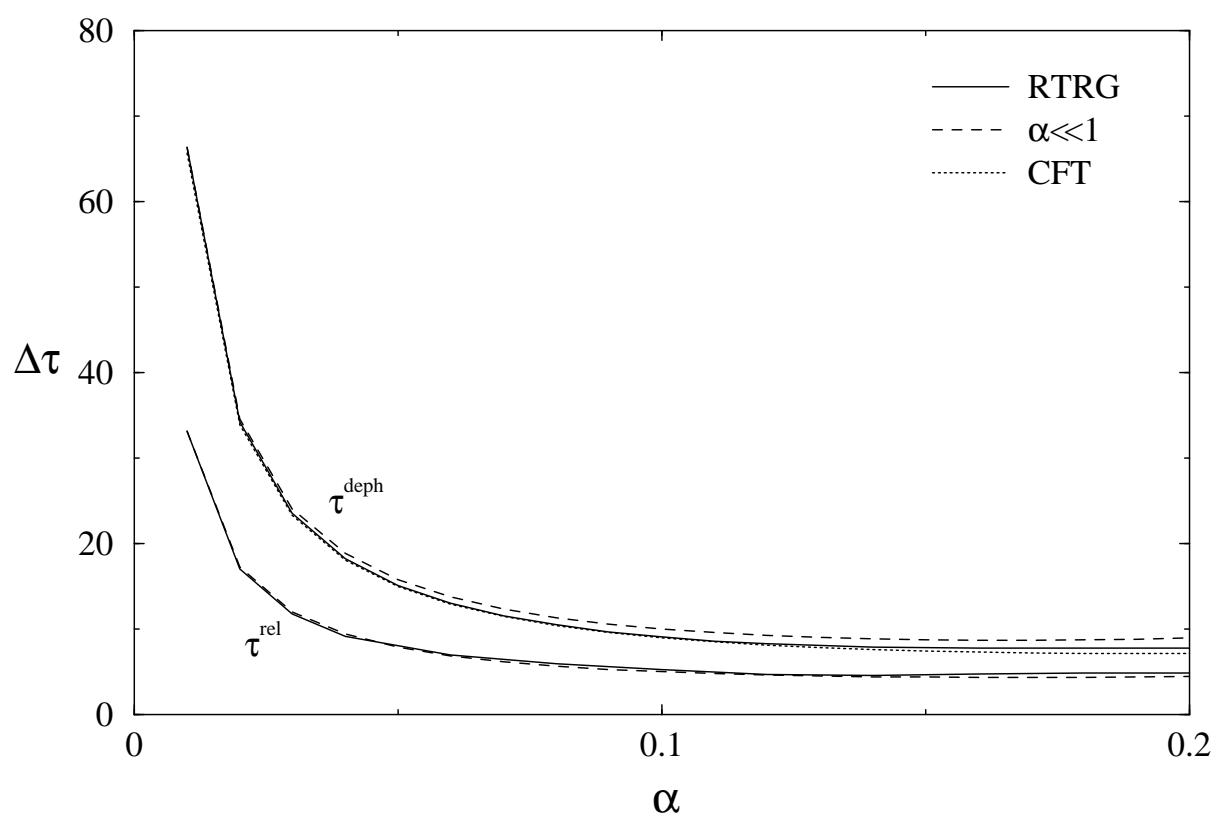

Figure 3.23: Decay constants $\tau^{d e p h}$ and $\tau^{\text {rel }}$ as a function of the coupling constant $\alpha$ for $\epsilon=0, D=100 \Delta$ and $T=0$. Solid line: RTRG. Dashed line: $\tau_{0}^{d e p h}$ and $\tau_{0}^{r e l}(\alpha \ll 1)$. Dotted line: $\tau_{C F T}^{\text {deph }}$.

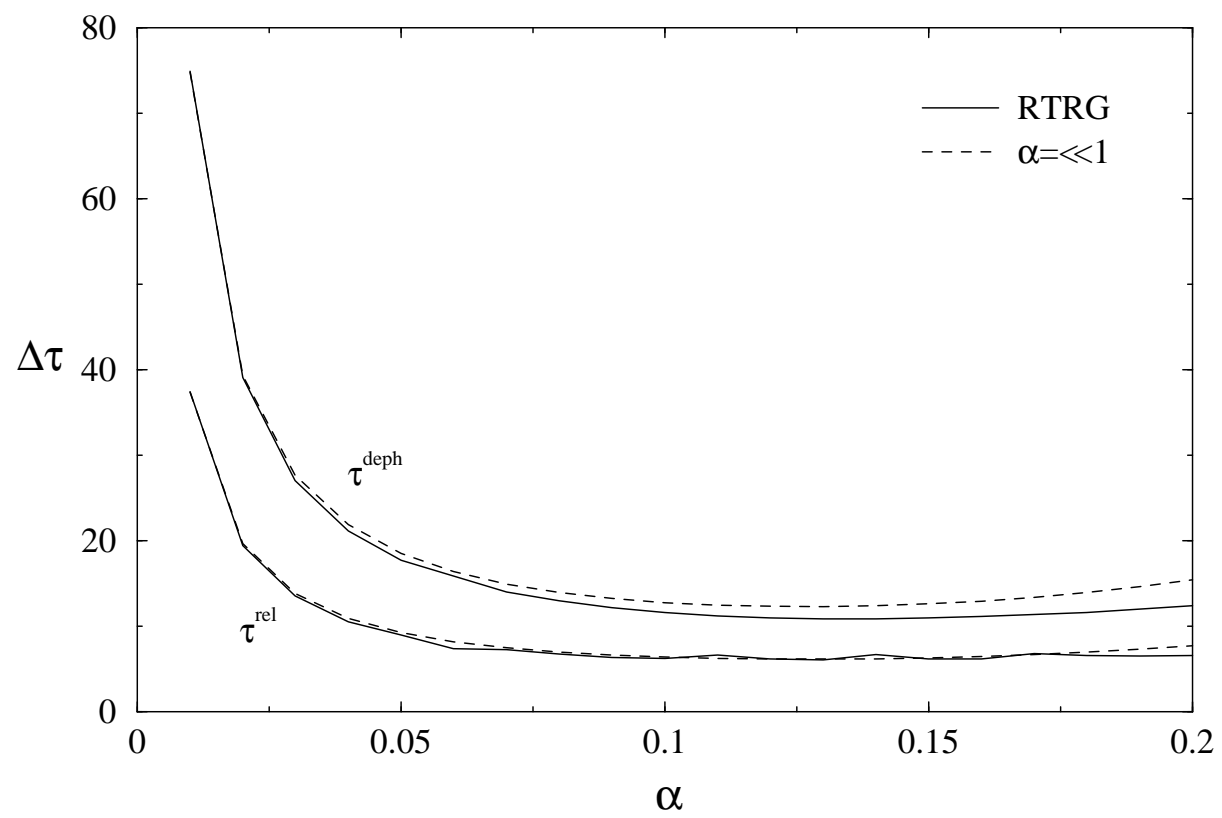

Figure 3.24: Decay constants $\tau^{\text {deph }}$ and $\tau^{r e l}$ as a function of the coupling constant $\alpha$ for $\epsilon=0.5 \Delta, D=100 \Delta$ and $T=0$. Solid line: RTRG. Dashed line: $\tau_{0}^{d e p h}$ and $\tau_{0}^{r e l}(\alpha \ll 1)$. 
For the symmetric case, a solution for the diagonal elements has been found using the CFT [29]. But as the diagonal elements exhibit a purely oscillating behaviour for $\epsilon=0$, CFT yields only a result for $\tau^{d e p h}$ :

$$
\begin{aligned}
\tau_{C F T}^{d e p h}= & \sin ^{-2}(\pi \alpha / 2(1-\alpha)) \frac{\sqrt{\pi} \Gamma(1 / 2(1-\alpha))}{\Gamma(\alpha / 2(1-\alpha))} \\
& \times\left(\frac{\Gamma\left(\frac{1}{2}+\alpha\right) \Gamma(1-\alpha) \Gamma(1-2 \alpha) \cos (\pi \alpha)}{\sqrt{\pi}}\right)^{1 / 2(\alpha-1)} \Delta_{r}^{-1}
\end{aligned}
$$

which again is valid for $0<\alpha<0.5$. We calculate the dephasing and relaxation times by studying the poles of $p(z)$. The $\alpha$ dependence of $\tau^{d e p h}$ and $\tau^{r e l}$ for the unbiased and the biased case is shown in Figs. 3.23 and 3.24. Again our results coincide with $\tau_{0}^{\text {deph }}$ and $\tau_{0}^{r e l}$ only in the limit of small $\alpha$, and the result of $\tau^{d e p h}$ for $\epsilon=0$ is in good agreement with CFT.

\subsubsection{The Shiba-relation}

The Shiba-relation is an exact equation for $T=0$, which was originally derived for the Anderson model [60]. In time space the relation determines the asymptotic long-time behaviour of the symmetrized equilibrium correlation function [1]:

$$
\begin{aligned}
C^{s}(t) & :=\frac{1}{2} \operatorname{Tr}\left[\left[\sigma_{z}(t), \sigma_{z}\right]_{+} \rho^{e q}\right]-\left(\operatorname{Tr}_{0}\left[\sigma_{z} p_{s t}\right]\right)^{2} \\
& =-2 \alpha \frac{\chi_{0}^{2}}{t^{2}} \quad \text { for } \quad t \rightarrow \infty
\end{aligned}
$$

Here, $[\cdot, \cdot]_{+}$again denotes the anticommutator.

In energy space we obtain for the Fourier transform

$$
C^{s}(\omega):=\int_{-\infty}^{\infty} d t e^{i \omega t} C^{s}(t)
$$

an equation for the low-frequency limit:

$$
C^{s}(\omega)=2 \pi \alpha \chi_{0}^{2}|\omega| \quad \text { for } \quad \omega \rightarrow 0 .
$$

When checking the RTRG results using the Shiba-relation, the discontinuity of $C^{s}(\omega) / \omega$ for $\omega \rightarrow 0$ leads to numerical instabilities. Therefore, we focus on the antisymmetrized equilibrium correlation function involving the commutator $[\cdot, \cdot]_{-}$. The imaginary part of the dynamic susceptibility is defined as

$$
\chi^{\prime \prime}(\omega)=\int_{-\infty}^{\infty} d t e^{i \omega t} \frac{1}{2} \operatorname{Tr}\left[\left[\sigma_{z}(t), \sigma_{z}\right]_{-} \rho^{e q}\right] .
$$

$\chi^{\prime \prime}(\omega)$ is connected with $C^{s}(\omega)$ by the fluctuation-dissipation theorem [1]

$$
C^{s}(\omega)=\operatorname{coth}\left(\frac{\omega}{2 T}\right) \chi^{\prime \prime}(\omega)
$$




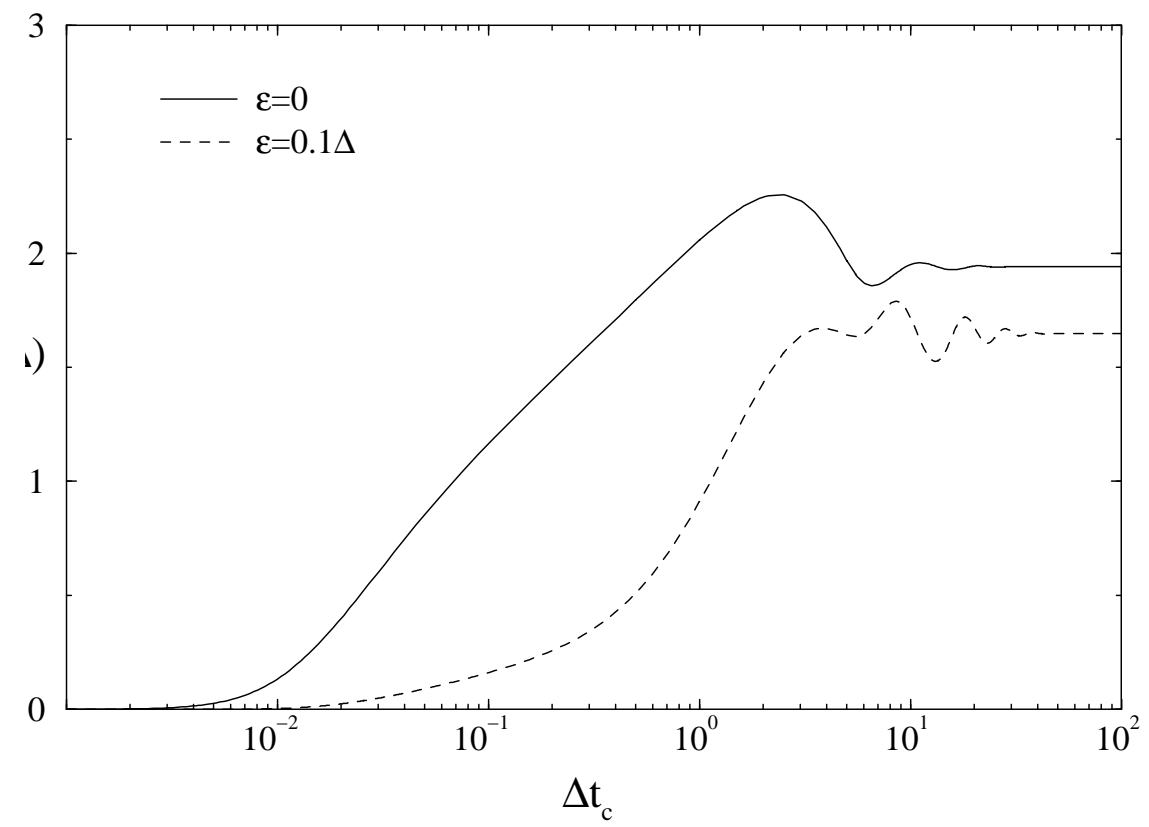

Figure 3.25: Spectral function $S(0.1 \Delta)$ as a function of $t_{c}$ for $\alpha=0.1, D=100 \Delta$ and $T=0$. Solid line: $\epsilon=0$. Dashed line: $\epsilon=0.1 \Delta$.

Hence, the Shiba-relation for the imaginary part of the dynamic susceptibility reads

$$
\chi^{\prime \prime}(\omega)=2 \pi \alpha \chi_{0}^{2} \omega \quad \text { for } \quad \omega \rightarrow 0 .
$$

We now use Eqs. (3.136) - (3.140), (3.144) and (3.145) to calculate $\chi^{\prime \prime}(\omega)$. Since Eq. (3.81) determines the Laplace transform of an equilibrium correlation function, we write

$$
\chi^{\prime \prime}(\omega)=\operatorname{Re}\left[\int_{0}^{\infty} d t e^{i \omega t} \operatorname{Tr}\left[\left[\sigma_{z}(t), \sigma_{z}\right]_{-} \rho^{e q}\right]\right] .
$$

In Figs. 3.25 and 3.26 one can see the $t_{c}$ dependence of the spectral function $S(\omega)=\chi^{\prime \prime}(\omega) / \omega$ at $\omega=0.1 \Delta$ for $D=100 \Delta, T=0$ and different values of $\epsilon$ and $\alpha$. We again use $\bar{t} \rightarrow \infty$, unless stated otherwise.

The asymptotic $\left(t_{c} \rightarrow \infty\right)$ result of $S$ as a function of $\omega$ is shown in Fig. 3.27 for $\alpha=0.1, T=0$ and different values of $D$ and $\epsilon$. As in case of the static susceptibility one obtains a strong $D$ dependence. But again one may normalize the result using $S(0)=\lim _{\omega \rightarrow 0} \chi^{\prime \prime}(\omega) / \omega$ and the frequency $\omega_{\max } \approx \Delta_{r}$, where $S$ is maximum. Then the curves for different cutoffs coincide, thereby showing the correct scaling behaviour (see Fig. 3.28, where $\epsilon$ has also been rescaled).

We test the Shiba-relation, Eq. (3.165), for different $\alpha, \epsilon$ and $D$ which can be seen in Tab. 3.1. For $\alpha \lesssim 0.1$, we achieve a very good agreement with the Shiba-relation (error smaller than 5\%). In comparison, the flow equation method of Wegner produces an error of $25 \%$ for $\alpha=0.1$ [66]. One recognizes, that for larger $\alpha$ our error increases strongly, which is due to our neglecting of double vertex objects. However, in case of $\alpha=0.2$ we 


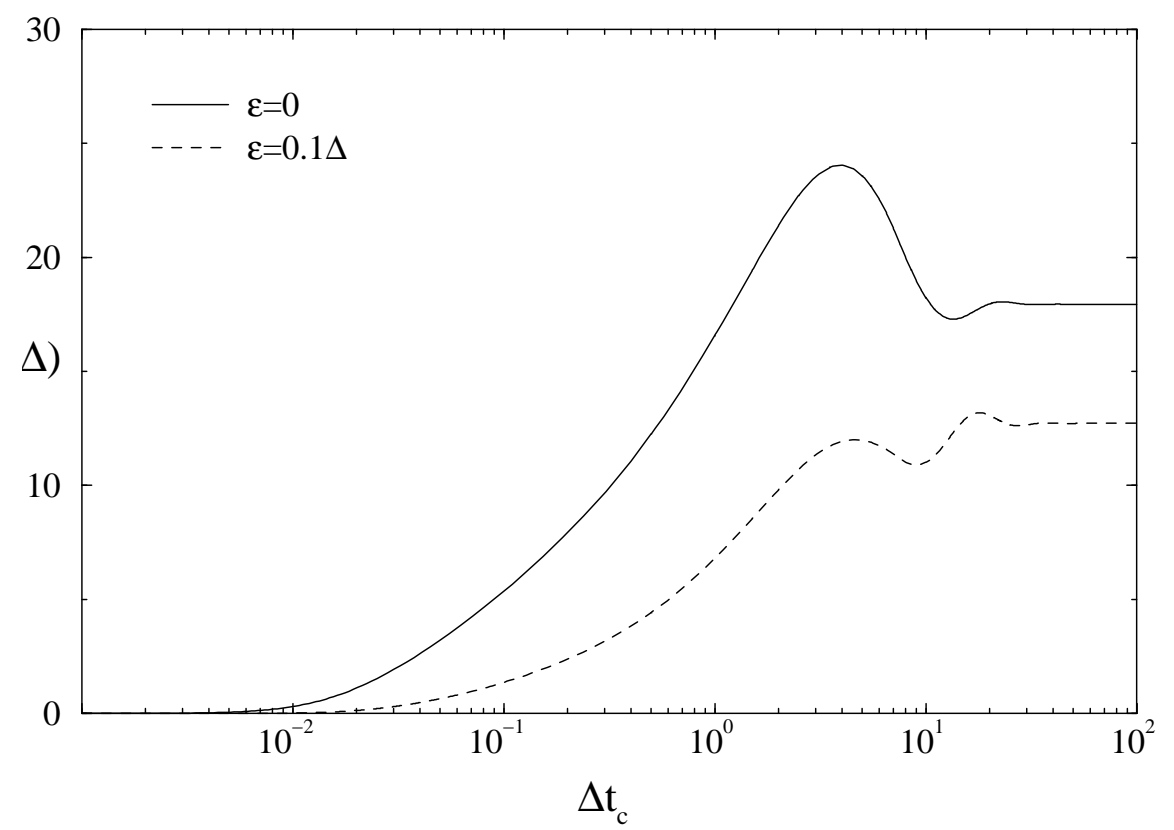

Figure 3.26: Spectral function $S(0.1 \Delta)$ as a function of $t_{c}$ for $\alpha=0.2, D=100 \Delta$ and $T=0$. Solid line: $\epsilon=0$. Dashed line: $\epsilon=0.1 \Delta$.

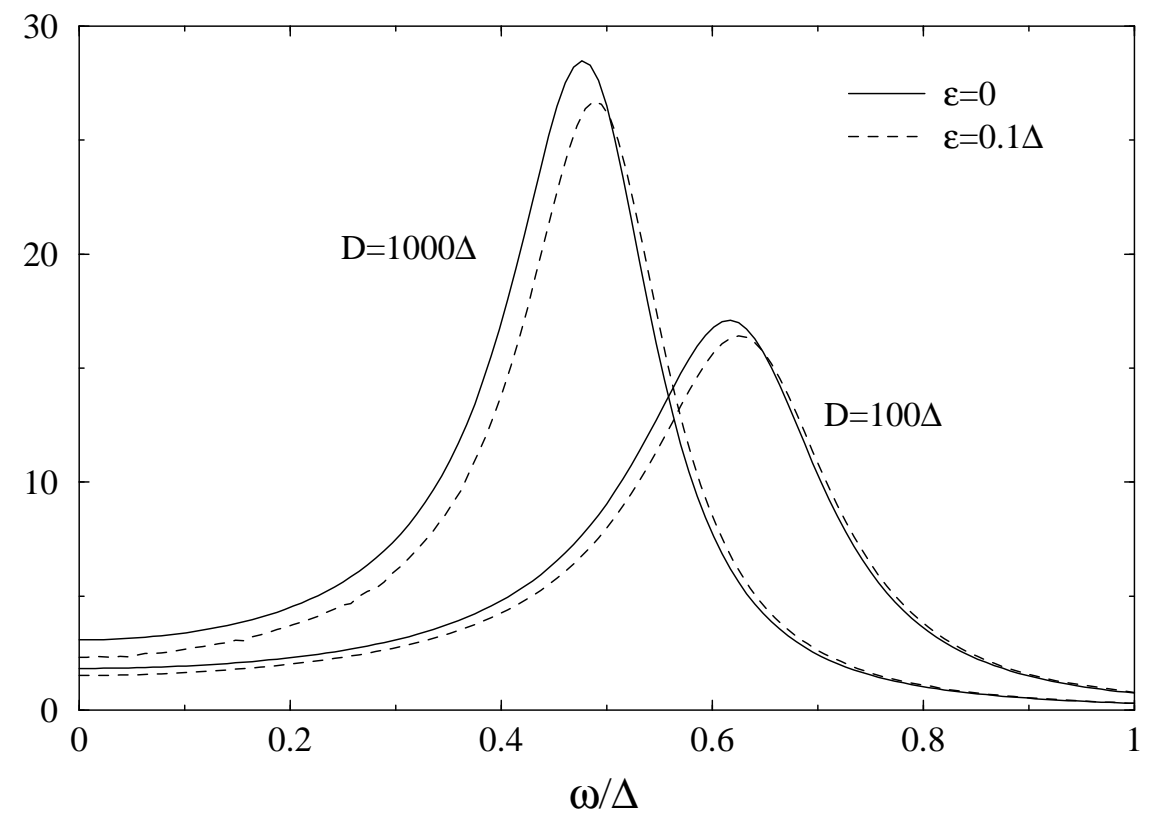

Figure 3.27: Spectral function $S$ as a function of frequency $\omega$ for $\alpha=0.1, T=0$ and $D=100 \Delta, 1000 \Delta$. Solid lines: $\epsilon=0$. Dashed lines: $\epsilon=0.1 \Delta$. 


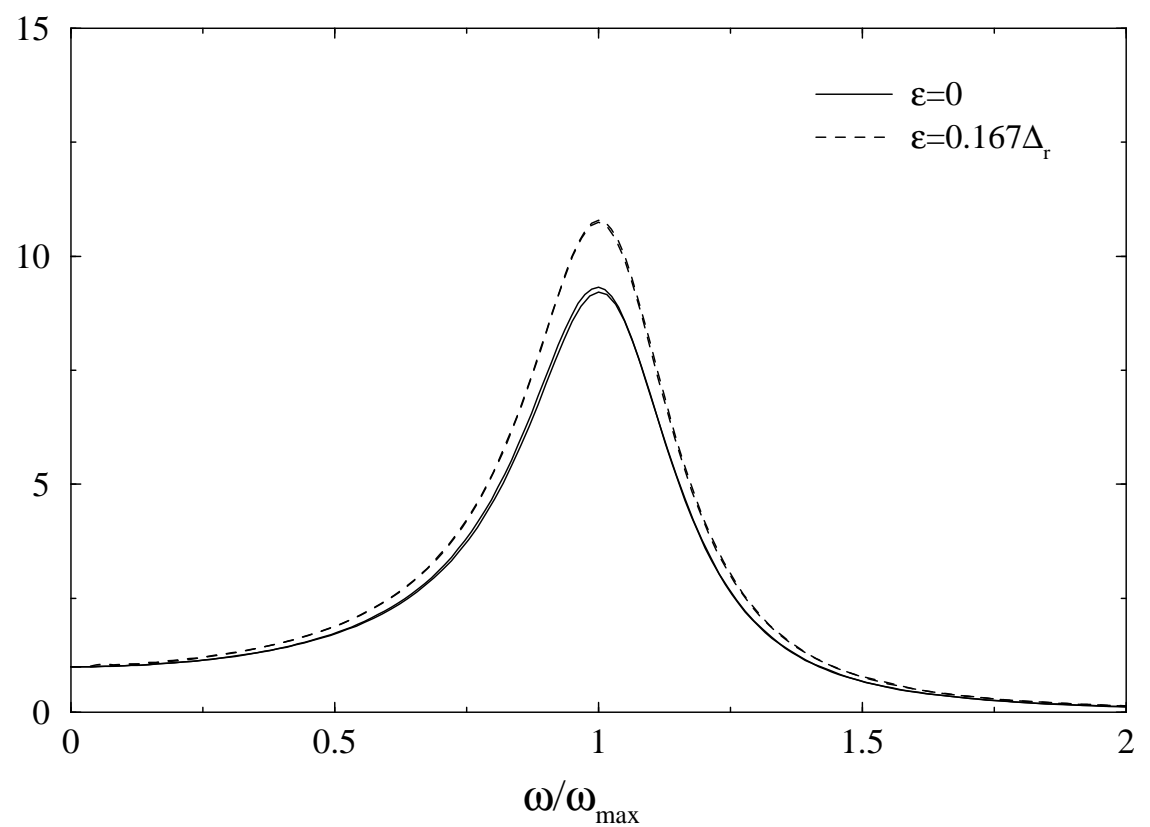

Figure 3.28: Rescaled spectral function $S$ as a function of frequency $\omega$ for $\alpha=0.1$, $D \gg \Delta_{r}$ and $T=0$. Solid lines: $\epsilon=0$. Dashed lines: $\epsilon=0.167 \Delta_{r}$.

\begin{tabular}{|l|l|r|r|r|r|r|}
\hline$\alpha$ & $\epsilon / \Delta$ & $D / \Delta$ & $\Delta \chi_{0}$ & $2 \pi \alpha\left(\Delta \chi_{0}\right)^{2}$ & $\lim _{\omega \rightarrow 0} \Delta^{2} S(\omega)$ & error \\
\hline 0.01 & 0.0 & 100 & 1.051 & 0.06942 & 0.06798 & $2.09 \%$ \\
0.05 & 0.0 & 100 & 1.290 & 0.5227 & 0.5083 & $2.79 \%$ \\
0.1 & 0.0 & 100 & 1.687 & 1.788 & 1.834 & $2.55 \%$ \\
0.2 & 0.0 & 100 & 2.995 & 11.27 & 15.54 & $31.85 \%$ \\
0.2 & 0.0 & 100 & $3.240^{*}$ & $13.19^{*}$ & $12.31^{*}$ & $6.90 \%{ }^{*}$ \\
0.01 & 0.0 & 1000 & 1.077 & 0.07291 & 0.07115 & $2.44 \%$ \\
0.05 & 0.0 & 1000 & 1.453 & 0.6636 & 0.6445 & $2.92 \%$ \\
0.1 & 0.0 & 1000 & 2.180 & 2.987 & 3.088 & $3.33 \%$ \\
0.2 & 0.0 & 1000 & 5.159 & 33.44 & 51.34 & $42.23 \%$ \\
0.2 & 0.0 & 1000 & $5.722^{*}$ & $41.14^{*}$ & $35.49^{*}$ & $14.75 \%$ \\
0.1 & 0.01 & 100 & 1.686 & 1.786 & 1.831 & $2.49 \%$ \\
0.1 & 0.05 & 100 & 1.667 & 1.746 & 1.752 & $0.34 \%$ \\
0.1 & 0.1 & 100 & 1.604 & 1.616 & 1.621 & $0.31 \%$ \\
0.1 & 0.01 & 1000 & 2.178 & 2.980 & 3.091 & $3.66 \%$ \\
0.1 & 0.05 & 1000 & 2.137 & 2.869 & 2.723 & $5.22 \%$ \\
0.1 & 0.1 & 1000 & 2.019 & 2.561 & 2.438 & $4.92 \%$ \\
\hline
\end{tabular}

Table 3.1: Shiba-relation for different $\alpha, \epsilon$ and $D$.

The star* indicates the finite value of $\bar{t}=1 / \Delta$. 


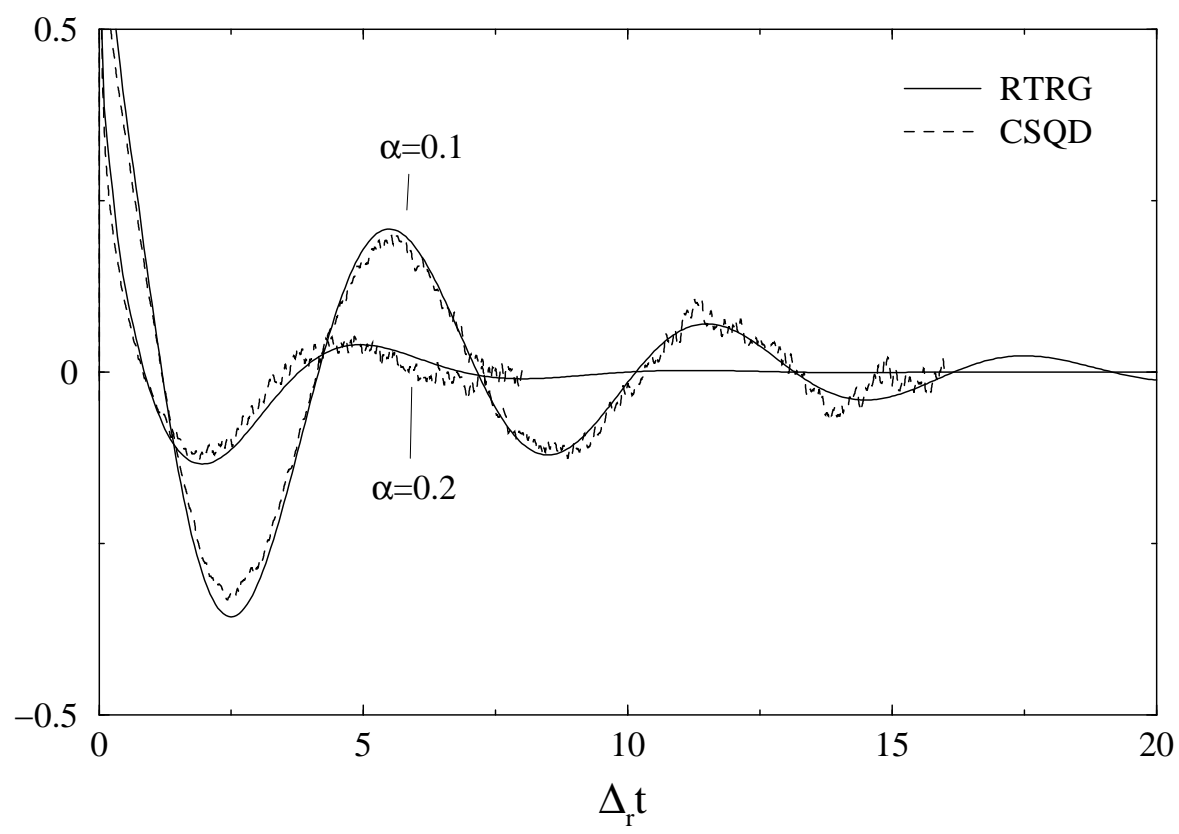

Figure 3.29: Correlation function $C_{y y}^{s}(t)$ for $\alpha=0.1,0.2, \epsilon=0, D=50 \Delta_{r}$ and $T=0$. Solid lines: RTRG. Dashed lines: CSQD.

also present the data obtained for a finite crossover parameter $\bar{t}=1 / \Delta$ (indicated by ${ }^{*}$ ). Then, the error is reduced substantially. In this context it must be noted again, that our results for the reduced density matrix $p(t)$ were independent of $\bar{t}$ within numerical errors.

Analogously to Subsection 3.4.3 we may also transform the results for the Laplace tranform of an equilibrium correlation function into time space. We present the time evolution of

$$
C_{y y}^{s}(t):=\frac{1}{2} \operatorname{Tr}\left[\left[\sigma_{y}(t), \sigma_{y}\right]_{+} \rho^{e q}\right] .
$$

In Fig. 3.29 our results for $\epsilon=0, D=50 \Delta_{r}, T=0$ and different $\alpha$ are compared to CSQD calculations [57]. We again find a good agreement.

\subsection{Discussion}

We studied the spin-boson model as a fundamental quantum dissipative system. It is applicable for any physical problem, where one effectively deals with two states, which are coupled to a bosonic heat reservoir. Thus it is a basic model which, however, contains the nontrivial features of quantum dissipation and, therefore is not exactly soluble. We have used a new RG approach, the real-time renormalization-group method to study the spin-boson model. Thereby we have been able to determine both static and dynamic properties. By calculating the time-dependent reduced density matrix, we have been able to examine the effects of quantum dissipation on the dynamics of a local system, when 
one starts out from an arbitrary nonequilibrium state. Previously such studies have not been possible within a (nonperturbative) RG approach.

The RTRG method has been explained in detail. It is formulated for the Keldysh contour, so that dissipation is properly taken into account, i.e. non-Hamiltonian dynamics is generated within this approach. Therefore any nonequilibrium situation may be considered. Furthermore, we have extended the formalism to the calculation of equilibrium correlation functions $[25,26]$.

We have found that the standard form of the RTRG (sharp cutoff for the bath contractions) generates unphysical terms for the spin-boson model. However, since the general formalism does not specify the $t_{c}$ dependence of the bath contractions explicitly, we could avoid those unphysical terms by applying a modified choice for $\gamma^{p_{1}, p_{2}}\left(t, t_{c}\right)$.

From the resulting RG equations we achieved reliable results for the whole parameter space with the only restriction of $\alpha \lesssim 0.1-0.2[25,26]$. We have shown, that, due to its nonperturbative nature the approach accounts for the correct renormalized tunnel matrix element. In contrast to the study of the polaron, the numerical solution of the RG equations exhibited a convergent behaviour for $t_{c} \rightarrow \infty$, since, here, the bath contractions were decaying. From the results for the stationary reduced density we determined the static susceptibility. Furthermore, we presented a solution for the complete dynamics of the two-state system for an arbitrary initial nonequilibrium preparation. The tunneling frequency $\Omega \sim \Delta_{r}$ as well as the decay constants in the asymptotic regime, the dephasing and the relaxation time, have been determined. Eventually, we have calculated equilibrium correlation functions both in energy and in time space.

We directly solved the spin-boson model for any parameter value of the high-energy cutoff $D$, the bias $\epsilon$, the tunneling $\Delta$, and the temperature $T$. Therefore, a quantitative and unambigious comparison to the anisotropic Kondo model could be drawn in a parameter regime, where the mapping of that model on the spin-boson model cannot be proven rigorously. We found, that for $\epsilon=0$ both the oscillation frequency $\Omega$ of the diagonal elements of $p(t)$ and the dephasing time $\tau^{d e p h}$ agree with the results of CFT, which were obtained for the anisotropic Kondo model. Furthermore, in the scaling limit the static susceptibility agrees rather well with Bethe ansatz results for the anisotropic Kondo model for $\epsilon=0$, but deviations occur at finite bias.

We have also shown that our method gives a much better description for the time evolution of the reduced density matrix than the NIBA, which, for vanishing temperature, is restricted to the diagonal elements for the unbiased case. In contrast to the NIBA, we have been able to study the complete dynamics of the reduced density matrix both for the unbiased and the biased case.

The generalization of the RTRG method to the calculation of equilibrium correlation functions allowed us the examination of the imaginary part of the dynamic susceptibility. Its low-frequency behaviour for vanishing temperature is connected with the static susceptibility by the exact Shiba-relation. Concerning this relation we obtained the very small error $\lesssim 5 \%$ for $\alpha \lesssim 0.1$. Additionally, we compared our result for the correlation function $C_{y y}^{s}(t)$ with CSQD and obtained a good agreement. We note, that, in contrast to other methods, the RTRG yields reliable results for the equilibrium correlation func- 
tions for the spin-boson model for $\alpha=0.1$. NRG results $[6,70]$ are very accurate for low frequency but fail for $\omega \sim \Delta$ [66], flow equation methods have already an error of $\sim 25 \%$ concerning the Shiba-relation [66], and CSQD does not provide a check of the Shiba-relation [57]. Generally, QMC methods have difficulties to determine the longtime respectively low-frequency behaviour of physical quantities due to the dynamical sign problem. Furthermore, the reliability of our method is also demonstrated by the consistency of the time-dependent reduced density matrix with CSQD and the correct scaling behaviour of our results. The restriction in $\alpha$ is due to the fact that we neglected double vertex objects. When one includes these objects in the approach, the method gets much more complicated. However, in Chapter 5 a possibility to account for double vertex objects will be explained.

Finally, we note that the derivation of the formalism in principle holds for any quantum dissipative system. The presented solution of the spin-boson model shows that the RTRG provides a powerful tool to study various kinds of quantum dissipative systems for any nonequilibrium situation. Among these systems, quantum dot structures have recently attracted both experimental and theoretical interest. In the next chapter we will study coupled quantum dots in a phonon environment. We will calculate the current through them, when an external bias gives rise to a nonequilibrium stationary state. 


\section{Chapter 4}

\section{Coupled quantum dots}

Today quantum dot systems allow a detailed study of many physical phenomena, like Coulomb blockade [76, 31], Kondo effects [77, 32, 33] or interference effects [32]. As in these structures quantum states can be manipulated, they also may have an application in future quantum gates [78]. Quantum dot systems can typically be characterized by only a few parameters, which are experimentally controllable. Theoretically, these systems may then be described by basic models, which capture the essential physics. In this chapter we present a theoretical analysis of an out-of-equilibrium quantum dot experiment, where an external voltage $V$ gives rise to a stationary tunnel current through a double quantum dot, which interacts with a phonon bath (see Fig. 4.1). The current was measured at low temperature as a function of the energy difference $\epsilon$ of the two dot levels and the influence of the phonon environment was examined [30]. The thermal energy of the environment is always a source of unwanted transitions in quantum dot devices. Even at zero temperature spontaneous emission of phonons gives rise to inelastic transitions, i.e. they occur between dot states of nonequal energy. In the experiment described in Ref. [30] the inelastic contribution to the tunnel current through the double dot was studied. A first theoretical interpretation of the experimental results focused on the interaction of the dots with the phonons, which is analogous to the spin-boson model [34]. The authors found the qualitative current spectrum by eliminating the coupling to the leads perturbatively, and applying an approximation to the electron-phonon problem, which corresponds to the noninteracting blip approximation (NIBA) for the spin-boson model [27]. However, a quantitative comparison with the experiment has not yet been possible. Especially, the unexpectedly large inelastic current of the experiment could not be explained.

As we have seen in Chapter 3, the RTRG provides a powerful method to study such a nonequilibrium problem for moderately strong couplings. Therefore, here we again apply the RTRG approach, thereby treating both the coupling to the phonon reservoir and the coupling to the leads nonperturbatively. Thus, we obtain a quantitatively reliable solution for the tunnel current through the double dot system for a wide range of coupling parameters, including those of the experiment of Ref. [30]. Both the level broadening induced by the coupling to the leads is included in this method, and the external voltage is accounted for properly. Moreover, we do not deal with the parameter restrictions of the 


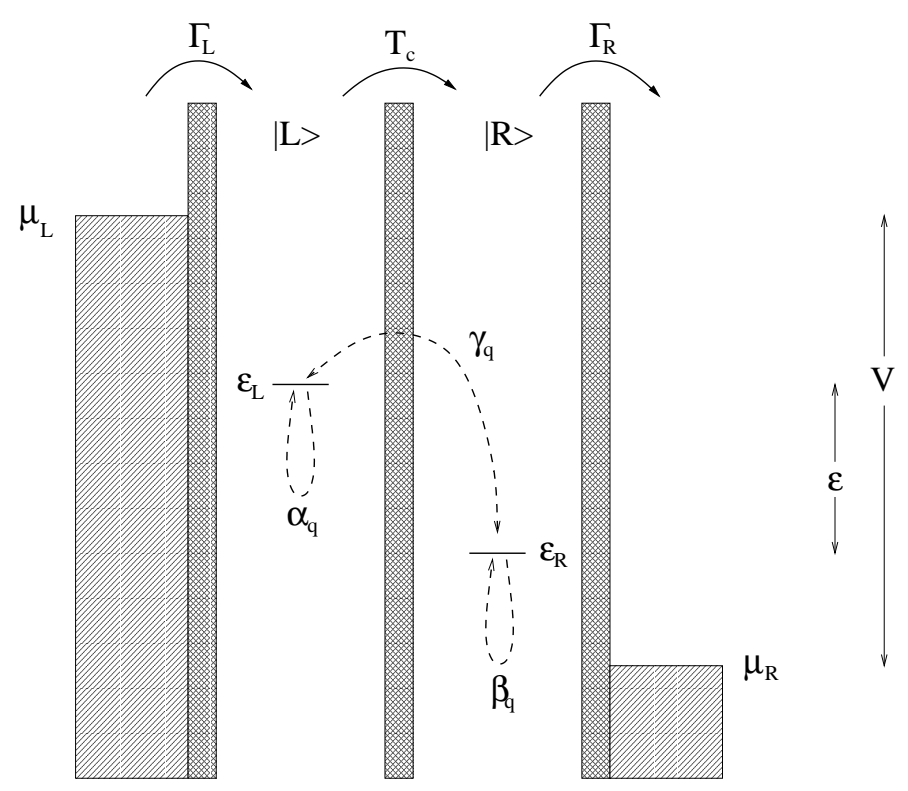

Figure 4.1: The double quantum dot may be charged with one additional electron in the left or right dot. The corresponding states $|L\rangle$ and $|R\rangle$ are coupled by the tunnel amplitude $T_{c}$. The couplings to the leads are given by $\Gamma_{L(R)}$. The energy difference between the quantum dots is $\epsilon=\epsilon_{L}-\epsilon_{R}$, and there is an external voltage $V=\mu_{L}-\mu_{R}$. The interaction with the acoustical phonons (dashed lines), consists of a diagonal part with the coefficients $\alpha_{q}$ and $\beta_{q}$, and an off-diagonal part with the constants $\gamma_{q}$.

NIBA, which, in this case is valid only for sufficiently high temperature. Note that the calculation of the tunnel current through the double dot as a function of $\epsilon$ corresponds to determining the off-diagonal elements of the reduced density matrix for the biased case of the spin-boson model, but we have seen in Chapter 3, that for this case the NIBA fails at low temperature. Additionally, the RTRG method may treat any form of dot-phonon interaction. Therefore, we are able to account for the full electron-phonon interaction, i.e. including interaction terms, which involve a tunneling between the dots ("off-diagonal interaction terms"). In the quantitative analysis the off-diagonal interaction terms lead to a strong dependence of the current on the extension of the wavefunctions within one dot. We find that the variation of this extension with the energy difference $\epsilon$ between the dot levels has to be accounted for. By fitting the result for the current with the experimental data, we obtain the width of the electron density within one dot as a function of $\epsilon$ [35].

\subsection{Model Hamiltonian}

Let us first derive a model Hamiltonian for the double dot system as it was realized in the experiment [30]. Our model consists of two coupled quantum dots ( $L$ and $R$, respectively). Each dot is coupled to an electron reservoir with the chemical potentials $\mu_{L}$ and $\mu_{R}$, see Fig. 4.1. In the experiment the external voltage $V=\mu_{L}-\mu_{R}$ was much smaller 
than the Coulomb charging energy $U$. Thus, due to Coulomb blockade the double dot cannot be charged with more than one additional electron. Furthermore, a strong magnetic field was applied perpendicular to the dots. Thus, we assume spin polarization here and omit the spin index. We denote the many-particle ground-state, where an additional electron is in the left (right) dot, by $|L\rangle(|R\rangle)$ and neglect any excited states. Therefore, together with the uncharged ground state $|0\rangle$, there are only three possible states of the double dot. The total Hamiltonian $\bar{H}$ for the system can be written as a sum of the dot Hamiltonian, the contributions of the electron reservoirs and the phonon bath, and the interaction parts stemming from the coupling to the leads and the electron-phonon interaction:

$$
\bar{H}=H_{d}+H_{r e s}+H_{p h}+H_{e-r e s}+H_{e-p h} .
$$

The dot Hamiltonian $H_{d}$ reads

$$
H_{d}=\epsilon_{L}|L\rangle\left\langle L\left|+\epsilon_{R}\right| R\right\rangle\langle R|+T_{c}(|L\rangle\langle R|+| R\rangle\langle L|)
$$

where $\epsilon_{L}\left(\epsilon_{R}\right)$ is the ground-state energy of $|L\rangle(|R\rangle)$ and the coupling between the dots is described by the tunnel matrix element $T_{c}$. The reservoir contributions are given by

$$
\begin{aligned}
H_{r e s} & =\sum_{k} \epsilon_{k} c_{k}^{\dagger} c_{k}+\sum_{k} \epsilon_{k} d_{k}^{\dagger} d_{k} \\
H_{p h} & =\sum_{q} \omega_{q} a_{q}^{\dagger} a_{q} .
\end{aligned}
$$

Here, the operator $c_{k}^{\dagger}\left(c_{k}\right)$ creates (annihilates) an electron with the energy $\epsilon_{k}$ in the left lead, whereas the creation (annihilation) operator $d_{k}^{\dagger}\left(d_{k}\right)$ refers to the right electron reservoir. Analogously, $a_{q}^{\dagger}\left(a_{q}\right)$ creates (annihilates) a phonon with the wave vector $\vec{q}$ and the frequency $\omega_{q}$. In this chapter we again choose units such that $\hbar=k_{B}=1$, furthermore we set the elementary charge $e=1$. The double dot is coupled to the external leads by the parameters $V_{k}$ and $W_{k}$ :

$$
\begin{aligned}
H_{e-r e s}= & \sum_{k}\left(V_{k} c_{k}|L\rangle\left\langle 0\left|+V_{k}^{*}\right| 0\right\rangle\langle L| c_{k}^{\dagger}\right) \\
& +\sum_{k}\left(W_{k} d_{k}|R\rangle\left\langle 0\left|+W_{k}^{*}\right| 0\right\rangle\langle R| d_{k}^{\dagger}\right) .
\end{aligned}
$$

The electron-phonon interaction consists of a diagonal part, which is characterized by the coupling constants $\alpha_{q}$ and $\beta_{q}$, and an off-diagonal contribution with the parameter $\gamma_{q}$ :

$$
\begin{aligned}
H_{e-p h}= & \sum_{q}\left(\alpha_{q}|L\rangle\left\langle L\left|+\beta_{q}\right| R\right\rangle\langle R|\right)\left(a_{q}^{\dagger}+a_{-q}\right) \\
& +\sum_{q} \gamma_{q}(|L\rangle\langle R|+| R\rangle\langle L|)\left(a_{q}^{\dagger}+a_{-q}\right) .
\end{aligned}
$$


The above interaction coefficients are given by [34]

$$
\begin{aligned}
\alpha_{q} & =\lambda_{q}\left\langle L\left|e^{i \vec{q} \vec{x}}\right| L\right\rangle, \\
\beta_{q} & =\lambda_{q}\left\langle R\left|e^{i \vec{q} \vec{x}}\right| R\right\rangle, \\
\gamma_{q} & =\lambda_{q}\left\langle L\left|e^{i \vec{q} \vec{x}}\right| R\right\rangle,
\end{aligned}
$$

where $\lambda_{q}$ is the matrix element for the interaction of 2DEG electrons with phonons. The phonons are assumed to be three-dimensional acoustical phonons [30]. It then follows for the interaction [34]

$$
\left|\lambda_{q}\right|^{2}=g \frac{\pi^{2} c_{s}^{2}}{V|\vec{q}|}
$$

and the dispersion reads

$$
\omega_{q}=c_{s}|\vec{q}|
$$

Here, we introduced $c_{s}$ as the speed of sound in the medium, $V$ as the volume of the crystal, and the dimensionless coupling constant $g$ [34]. For the evaluation of Eqs. (4.7) - (4.9) we model the electron density $\rho_{L}(\vec{x})\left(\rho_{R}(\vec{x})\right)$ within one dot by a Gaussian, which is peaked around the dot position $\vec{x}_{L}\left(\vec{x}_{R}=\vec{x}_{L}+\vec{d}\right)$ with a width $|\Delta \vec{x}|=\sqrt{3 / 2} \sigma$ [35]:

$$
\rho_{L(R)}(\vec{x})=\left(\frac{1}{\pi \sigma^{2}}\right)^{3 / 2} e^{-\frac{\left(\vec{x}-\vec{x}_{L(R)}\right)^{2}}{\sigma^{2}}} .
$$

The finite width $\sigma$ leads to a high-energy cutoff $D=c_{s} / \sigma$ for the coefficients $\alpha_{q}, \beta_{q}$ and $\gamma_{q}$. We include this cutoff in an exponential form, so that we end up with the following interaction coefficients:

$$
\begin{aligned}
& \alpha_{q}=\lambda_{q} e^{i \vec{q} \vec{x}_{L}} e^{-\frac{c_{s}|\vec{q}|}{2 D}} \\
& \beta_{q}=\lambda_{q} e^{i \vec{q} \vec{x}_{R}} e^{-\frac{c_{s}|\vec{q}|}{2 D}} \\
& \gamma_{q}=\lambda_{q} e^{i \vec{q}\left(\frac{\vec{x}_{L}+\vec{x}_{R}}{2}\right)} e^{-\frac{|\vec{d}| D}{2 c_{s}}} e^{-\frac{c_{s}|\vec{q}|}{2 D}} .
\end{aligned}
$$

A simple form of the Hamiltonian, which shows the analogy with the spin-boson model, is obtained by shifting the bosonic field operators. One introduces the unitary transformation 


$$
U=\exp \left[\sum_{q}\left(\frac{\alpha_{q}+\beta_{q}}{2 \omega_{q}} a_{q}^{\dagger}-\frac{\alpha_{q}^{*}+\beta_{q}^{*}}{2 \omega_{q}} a_{q}\right)\right]
$$

so that

$$
U a_{q} U^{\dagger}=a_{q}-\frac{\alpha_{q}+\beta_{q}}{2 \omega_{q}}
$$

Thus, our final Hamiltonian $H=U \bar{H} U^{\dagger}$ reads

$$
\begin{aligned}
H= & H_{0}+H_{B}+H_{V}, \\
H_{0}= & \frac{\epsilon}{2}(|L\rangle\langle L|-| R\rangle\langle R|) \\
& +T_{c}^{\mathrm{eff}}(|L\rangle\langle R|+| R\rangle\langle L|) \\
& +E(|L\rangle\langle L|+| R\rangle\langle R|-| 0\rangle\langle 0|), \\
H_{B}= & \sum_{k} \epsilon_{k} c_{k}^{\dagger} c_{k}+\sum_{k} \epsilon_{k} d_{k}^{\dagger} d_{k}+\sum_{q} \omega_{q} a_{q}^{\dagger} a_{q}, \\
H_{V}= & \sum_{\mu}: g_{\mu} j_{\mu}:,
\end{aligned}
$$

where we have used Eqs. (4.10), (4.11) and (4.13) - (4.15). Furthermore, for simplicity we have set $\left(\epsilon_{L}+\epsilon_{R}\right) / 2=0$ and introduced the parameters

$$
\begin{aligned}
\epsilon & =\epsilon_{L}-\epsilon_{R}, \\
T_{c}^{\mathrm{eff}} & =T_{c}-2 g \omega_{d} e^{-\frac{D}{2 \omega_{d}}} \arctan \frac{D}{2 \omega_{d}}, \\
E & =-\frac{g}{4}\left(D+\omega_{d} \arctan \frac{D}{\omega_{d}}\right)
\end{aligned}
$$

and

$$
\omega_{d}=\frac{c_{s}}{|\vec{d}|}
$$

Thus, the tunnel amplitude $T_{c}$ has to be replaced by a smaller effective $T_{c}^{\text {eff }}$, which is due to the off-diagonal electron-phonon interaction. One already recognizes that the reduction of $T_{c}$ strongly depends on the width of the electron densities $\sigma=|\vec{d}| \omega_{d} / D$. Finally, in view of the RTRG method, we have written the interaction part $H_{V}$ as normal ordered 
products of local (dot) operators $g_{\mu}$ and environmental operators $j_{\mu}$. They are defined by

$$
\begin{aligned}
g_{b_{1}} & =\frac{1}{2}(|L\rangle\langle L|-| R\rangle\langle R|), \\
j_{b_{1}} & =\sum_{q}\left(\alpha_{q}-\beta_{q}\right)\left(a_{q}^{\dagger}+a_{-q}\right), \\
g_{b_{2}} & =(|L\rangle\langle R|+| R\rangle\langle L|), \\
j_{b_{2}} & =\sum_{q} \gamma_{q}\left(a_{q}^{\dagger}+a_{-q}\right), \\
g_{b_{3}} & =-\frac{1}{2}|0\rangle\langle 0|, \\
j_{b_{3}} & =\sum_{q}\left(\alpha_{q}+\beta_{q}\right)\left(a_{q}^{\dagger}+a_{-q}\right), \\
g_{+L} & =g_{-L}^{\dagger}=|L\rangle\langle 0| \quad, \quad j_{+L}=j_{-L}^{\dagger}=\sum_{k} V_{k} c_{k}, \\
g_{+R} & =g_{-R}^{\dagger}=|R\rangle\langle 0| \quad, \quad j_{+R}=j_{-R}^{\dagger}=\sum_{k} W_{k} d_{k} .
\end{aligned}
$$

Therefore, the interaction index $\mu$ runs over the bosonic indices $b_{1}, b_{2}, b_{3}$ and the fermionic ones $+L,-L,+R,-R$. From Eqs. (4.18) - (4.21) a spin-boson model is recovered by omitting the electron reservoirs and the interaction with them, excluding the state $|0\rangle$ and neglecting the off-diagonal electron-phonon interaction, i.e. $\gamma_{q}=0$, which also means $T_{c}^{\mathrm{eff}}=T_{c}$. The correspondence to the spin-boson model, defined in Eqs. (3.2), (3.5) and (3.6), is then given by $T_{c}=-\Delta / 2$. Note that $E$ is then only a constant energy, which can be neglected. Although in this case the electron-phonon interaction is not exactly of the type of an Ohmic bath, we will see below in Subsection 4.2.2, that there is a strong relation to the standard spin-boson model in the Ohmic case with the correspondence of the coupling constants $g=2 \alpha$.

A solution for the stationary tunnel current in case of vanishing electron-phonon coupling $(g=0)$ was found by Stoof and Nazarov [79]. If one introduces the tunneling densities of states as

$$
\begin{aligned}
& \Gamma_{L}(\epsilon)=2 \pi \sum_{k}\left|V_{k}\right|^{2} \delta\left(\epsilon-\epsilon_{k}\right), \\
& \Gamma_{R}(\epsilon)=2 \pi \sum_{k}\left|W_{k}\right|^{2} \delta\left(\epsilon-\epsilon_{k}\right),
\end{aligned}
$$

and assumes, that $\Gamma_{L(R)}(\epsilon) \approx$ const. holds, the result reads

$$
I_{s t}=\frac{T_{c}^{2} \Gamma_{R}}{T_{c}^{2}\left(2+\Gamma_{R} / \Gamma_{L}\right)+\Gamma_{R}^{2} / 4+\epsilon^{2}} .
$$

Note that this formula deviates from the expressions given in both Ref. [30] and Ref. [34], which is due to the fact that the results given therein are incorrect. In Ref. [30] $\epsilon$ must 
be divided by $\hbar$ instead of $h$ [80], and in Ref. [34] a factor of 2 must be taken out of the definition of the rates $\Gamma_{f}$ to be consistent with the literature and with the results presented therein [81].

The influence of the electron-phonon interaction was qualitatively studied by using an approximation, which corresponds to the NIBA for the spin-boson model [34]. Thereby it was shown that the interference of phonons interacting with the electron densities at the two dots leads to an oscillating structure in the current spectrum. However, they treated the interaction with the electron reservoirs only perturbatively. Therefore, they had to introduce an additional cutoff parameter, which simulates the level broadening due to the coupling to the leads. Furthermore, they considered the limit of large external voltage $(V \rightarrow \infty)$, so that they could not study the regime $\epsilon \approx V$. Additionally, we have already mentioned that the calculation of the tunnel current as a function of the energy difference $\epsilon$ corresponds to determining the off-diagonal elements of the reduced density matrix for the spin-boson model for arbitrary bias $\epsilon$. Thus, this approach is only valid for the parameter regime $\left(\Delta_{r}^{2}+\epsilon^{2}\right)^{1 / 2} \lesssim T$ (see Appendix A). Here, $\Delta_{r}=\Delta(\Delta / D)^{\alpha /(1-\alpha)}$ is the renormalized tunnel amplitude for the spin-boson model (Eq. (3.11)). Finally, this approximation did not account for the off-diagonal terms of the electron-phonon interaction. Therefore, this analysis could not provide a quantitative result for $I_{s t}(\epsilon)$.

\subsection{The tunnel current within the RTRG}

We apply the RTRG to determine the stationary tunnel current through the double dot system. It is defined by

$$
I_{s t}=\lim _{t \rightarrow \infty}\langle I\rangle(t)
$$

where $I$ is the current operator

$$
I=i \sum_{k}\left(V_{k}^{*}|0\rangle\left\langle L\left|c_{k}^{\dagger}-V_{k} c_{k}\right| L\right\rangle\langle 0|\right)
$$

To calculate $I_{s t}$ it is not sufficient to determine the kernel $\Sigma$ introduced in Section 3.2. However, the scheme for the calculation of the reduced density matrix, which we have derived in Section 3.2, can easily be modified to also determine expectation values of operators, which are linear in the bath field operators [12]. Before we set up the RG equations, we again derive an exact expression for $I_{s t}$, which now depends on a kernel $\Sigma_{I}$.

\subsubsection{Exact expression for the stationary tunnel current}

First, we again introduce the Liouvillian $L=L_{0}+L_{B}+L_{V}$, which is defined as in Eq. (3.21). It acts on an operator $O$ according to

$$
L O=[H, O]_{-}\left(L_{0} O=\left[H_{0}, O\right]_{-}, \quad L_{B} O=\left[H_{B}, O\right]_{-}, \quad L_{V} O=\left[H_{V}, O\right]_{-}\right) \text {. }
$$


Similarly as in Eq. (3.22) the time-dependent expectation value can be written as

$$
\begin{aligned}
\langle I\rangle(t) & =\operatorname{Tr}_{0} \operatorname{Tr}_{B}\left[I e^{-i L t} p(0) \rho_{B}^{e q}\right] \\
& =\operatorname{Tr}_{0} \operatorname{Tr}_{B}\left[\left(-i A_{I}\right) e^{-i L t} p(0) \rho_{B}^{e q}\right] .
\end{aligned}
$$

Here we again assumed a factorized initial density matrix with $p(0)$ as the initial dot density matrix and $\rho_{B}^{e q}$ as the equilibrium distribution of the electron reservoirs and the phonon bath. Furthermore, we introduced the superoperator $A_{I}$ as

$$
A_{I} O=\left[\frac{i}{2} I, O\right]_{+},
$$

where $O$ again is an usual operator and $[\cdot, \cdot]_{+}$denotes the anticommutator. As in Section 3.2 we now use the interaction picture with respect to $L_{0}+L_{B}$ and obtain

$$
\langle I\rangle(t)=\operatorname{Tr}_{0} \operatorname{Tr}_{B}\left[e^{-i L_{0} t} T\left(-i A_{I}(t)\right) e^{-i \int_{0}^{t} d t^{\prime} L_{V}\left(t^{\prime}\right)} p(0) \rho_{B}^{e q}\right],
$$

where $T$ again denotes the time ordering operator. Expanding the above expression in $L_{V}$ and performing the trace over the bath degrees of freedom using Wick's theorem we can use the same diagrammatic language as in Section 3.2. According to Eq. (4.21) the interaction part $L_{V}$ can be written as

$$
L_{V}=\sum_{\mu, p= \pm}: G_{\mu}^{p} J_{\mu}^{p}:,
$$

where the superoperators $G_{\mu}^{p}$ and $J_{\mu}^{p}$ act on an operator $O$ :

$$
\begin{aligned}
G_{\mu}^{+} O & =g_{\mu} O, \\
G_{\mu}^{-} O & =-O g_{\mu}, \\
J_{\mu}^{+} O & =j_{\mu} O, \\
J_{\mu}^{-} O & =O j_{\mu} .
\end{aligned}
$$

Furthermore, the vertex $A_{I}$ can be written as

$$
A_{I}=\sum_{\mu, p= \pm}: A_{I \mu}^{p} J_{\mu}^{p}:
$$

where we introduced

$$
\begin{aligned}
& A_{I \mu}^{+} O=\frac{1}{2}\left(\delta_{\mu,+L}|L\rangle\left\langle 0\left|-\delta_{\mu,-L}\right| 0\right\rangle\langle L|\right) O \\
& A_{I \mu}^{-} O=\frac{1}{2} O\left(\delta_{\mu,+L}|L\rangle\left\langle 0\left|-\delta_{\mu,-L}\right| 0\right\rangle\langle L|\right) .
\end{aligned}
$$

Thus, we again obtain an effective theory for the local system, where the vertices $G_{\mu}^{p}$ and $A_{I \mu}^{p}$ of the local system are connected by the pair contractions

$$
\gamma_{\mu_{1}, \mu_{2}}^{p_{1}, p_{2}}(t)=\operatorname{Tr}_{B}\left[J_{\mu_{1}}^{p_{1}}(t) J_{\mu_{2}}^{p_{2}} \rho_{B}^{e q}\right]
$$




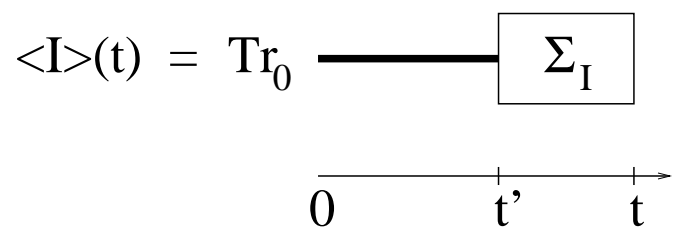

Figure 4.2: Diagrammatic expression for $\langle I\rangle(t)$. The two lines of the Keldysh contour are put together to one line. The irreducible diagrams in $\Sigma_{I}$ include the leftmost vertex superoperator at the time point $t^{\prime}$ and the current superoperator $A_{I \mu}^{p}$ at the time point $t . \Sigma_{I}$ acts on $p\left(t^{\prime}\right)$, which is represented by the thick horizontal line.

Introducing the kernel $\Sigma\left(t_{1}-t_{2}\right)$ as in Section 3.2, we again have to account for all possible sequences of such $\Sigma$ blocks, which can be expressed by $p(t)$. However, for the calculation of $\langle I\rangle(t)$ the last vertex of the last irreducible block is $A_{I \mu}^{p}$. We call that new object $\Sigma_{I}$, see Fig. 4.2. Finally, we also have to perform the trace over the local degrees of freedom. Formally, this yields [35]

$$
\langle I\rangle(t)=\operatorname{Tr}_{0}\left[\int_{0}^{t} d t^{\prime} \Sigma_{I}\left(t-t^{\prime}\right) p\left(t^{\prime}\right)\right]
$$

where the kernel is given by

$$
\Sigma_{I}\left(t_{1}-t_{2}\right)=(-i)^{2} \operatorname{Tr}_{B}\left[A_{I} e^{-i L_{0} t_{1}} T e^{-i \int_{t_{2}}^{t_{1}} d t L_{V}(t)} e^{i L_{0} t_{2}} L_{V} \rho_{B}^{e q}\right]_{\text {irred. }} .
$$

In addition to Eq. (3.31) we introduce the Laplace transforms

$$
\langle I\rangle(z)=\int_{0}^{\infty} d t e^{i z t}\langle I\rangle(t), \quad \Sigma_{I}(z)=\int_{0}^{\infty} d t e^{i z t} \Sigma_{I}(t)
$$

for $\operatorname{Im} z>0$. This leads to

$$
\langle I\rangle(z)=\operatorname{Tr}_{0}\left[\Sigma_{I}(z) p(z)\right]
$$

Using $I_{s t}=-i \lim _{z \rightarrow 0} z\langle I\rangle(z)$ together with Eq. (3.33), we finally obtain the exact equation

$$
I_{s t}=\operatorname{Tr}_{0}\left[\Sigma_{I}(z=0) p_{s t}\right] .
$$

The stationary reduced density matrix $p_{s t}$ can again be calculated from Eq. (3.34):

$$
\left[L_{0}+i \Sigma(z=0)\right] p_{s t}=0 .
$$

Thus, for the calculation of $I_{s t}$ we need to determine the kernels $\Sigma(z=0)$ and $\Sigma_{I}(z=0)$.

\subsubsection{RG equations for the coupled quantum dots}

For the calculation of $\Sigma(z=0)$ we use the framework given by Eqs. (3.60) - (3.64). Note that we now have the additional bath indices $\mu$, which the vertex superoperators 


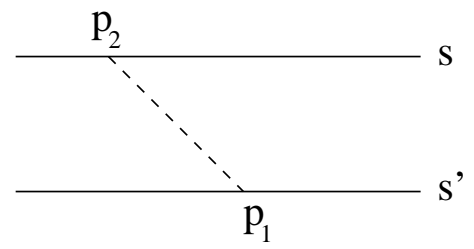

Figure 4.3: Additional sign arises for fermionic contractions, if the difference between the fermionic occupation numbers of the states $s$ and $s^{\prime}$ is odd, and $p_{1} \neq p_{2}$.
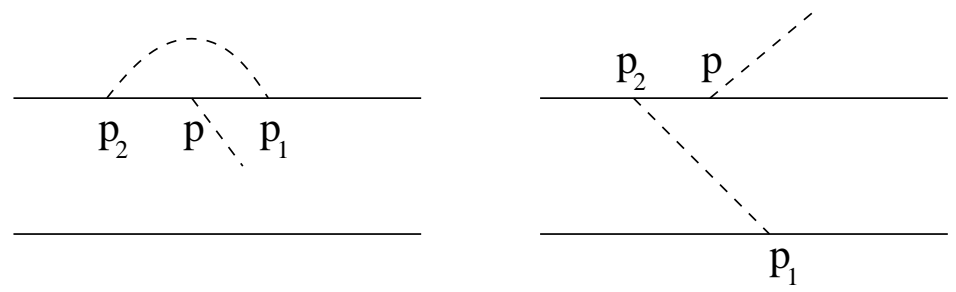

Figure 4.4: Additional sign arises for fermionic contractions, if the free vertex is fermionic, and $p=p_{2}$.

$G_{\mu}^{p}, A_{(I) \mu}^{p}$ and $B_{\mu}^{p}$ as well as the bath contractions $\gamma_{\mu_{1}, \mu_{2}}^{p_{1}, p_{2}}(t)$ depend on. Thus, we have $\mathrm{RG}$ equations for the vertices for each $\mu$, and the right-hand sides of the RG equations have to be summed over all possible contractions. Furthermore, we have to account for additional signs arising from the commutation of fermionic field operators [12]. There are two possible situations, where a fermionic contraction leads to a minus sign on the right-hand side of the RG equations, see Figs. 4.3 and 4.4. First, in Fig. 4.3 a minus sign occurs, when a fermionic contraction connects the upper and the lower propagator and the fermionic occupation numbers of the states $s$ and $s^{\prime}$ differ by an odd number. We account for this by including a matrix $\hat{\sigma}^{p_{1}, p_{2}}$ after the later vertex, i.e. in the RG equations we multiply the later vertex superoperator from the left with $\hat{\sigma}^{p_{1}, p_{2}}$, which is given by

$$
\left(\hat{\sigma}^{p_{1}, p_{2}}\right)_{s s^{\prime}, s s^{\prime}}=\left\{\begin{array}{cc}
p_{1} p_{2} & \text { for } N_{s}-N_{s^{\prime}}=\text { odd } \\
1 & \text { for } N_{s}-N_{s^{\prime}}=\text { even }
\end{array} .\right.
$$

Since we include the matrix $\hat{\sigma}^{p_{1}, p_{2}}$ after the later contraction vertex an additional minus sign occurs, when a fermionic vertex lies between the contraction vertices, and on the same propagator as the first contraction vertex (Fig. 4.4). This is accounted for by the function

$$
\eta_{\mu}^{p_{1}, p_{2}}=\left\{\begin{array}{cc}
-p_{1} p_{2} & \text { for } \mu \text { fermionic } \\
1 & \text { else }
\end{array} .\right.
$$

Regarding $\Sigma_{I}$ the only difference to $\Sigma$ is that the last vertex is not a usual vertex but the current vertex $A_{I \mu}^{p}$. Thus, we replace the right boundary vertex $A_{\mu}^{p}$ by $A_{I \mu}^{p}$ in Eq. (3.64) to obtain the RG equation for $\Sigma_{I}$. The renormalization of the current vertex $A_{I \mu}^{p}$ is completely analogous to Eq. (3.62), so that we again only have to replace $A_{\mu}^{p}$ by $A_{I \mu}^{p}$ to obtain 
the corresponding RG equation. However, note that the object $A_{I \mu}^{p}$ cannot be derived from $A_{\mu}^{p}$, since the initial conditions differ, see Eq. (3.43) and Eq. (4.48).

Let us now consider the explicit form of the bath contractions $\gamma_{\mu_{1}, \mu_{2}}^{p_{1}, p_{2}}(t)$. The bosonic contractions can be derived using Eqs. (4.13) - (4.15). As mentioned earlier they are strongly related to the bath contraction of the spin-boson model given by Eqs. (3.18) (3.20) with the correspondence of the coupling constants: $g=2 \alpha$. We again consider the case $D \gg T$, and therefore, we define

$$
\gamma_{S B M}^{p_{1}, p_{2}}(t)=-g\left(\operatorname{Re}\left[(\pi T)^{2} \frac{1}{\sinh ^{2}(\pi T(t-i / D))}\right]+i p_{2} \operatorname{Im}\left[\frac{1}{(t-i / D)^{2}}\right]\right) .
$$

Then, the bosonic contractions for the double dot system in the phonon bath read

$$
\begin{aligned}
\gamma_{b 1, b 1}^{p_{1}, p_{2}}(t) & =\gamma_{S B M}^{p_{1}, p_{2}}(t)-\frac{\omega_{d}}{2} \int_{t-1 / \omega_{d}}^{t+1 / \omega_{d}} d t^{\prime} \gamma_{S B M}^{p_{1}, p_{2}}\left(t^{\prime}\right) \\
\gamma_{b 1, b 2}^{p_{1}, p_{2}}(t) & =\gamma_{b 2, b 1}^{p_{1}, p_{2}}(t)=0 \\
\gamma_{b 1, b 3}^{p_{1}, p_{2}}(t) & =\gamma_{b 3, b 1}^{p_{1}, p_{2}}(t)=0 \\
\gamma_{b 2, b 2}^{p_{1}, p_{2}}(t) & =\frac{1}{2} e^{-D / \omega_{d}} \gamma_{S B M}^{p_{1}, p_{2}}(t), \\
\gamma_{b 2, b 3}^{p_{1}, p_{2}}(t) & =\gamma_{b 3, b 2}^{p_{1}, p_{2}}(t)=\omega_{d} e^{-D / 2 \omega_{d}} \int_{t-1 / 2 \omega_{d}}^{t+1 / 2 \omega_{d}} d t^{\prime} \gamma_{S B M}^{p_{1}, p_{2}}\left(t^{\prime}\right), \\
\gamma_{b 3, b 3}^{p_{1}, p_{2}}(t) & =\gamma_{S B M}^{p_{1}, p_{2}}(t)+\frac{\omega_{d}}{2} \int_{t-1 / \omega_{d}}^{t+1 / \omega_{d}} d t^{\prime} \gamma_{S B M}^{p_{1}, p_{2}}\left(t^{\prime}\right) .
\end{aligned}
$$

The above integral can be performed:

$$
\begin{aligned}
\int_{t-1 / \omega_{d}}^{t+1 / \omega_{d}} d t^{\prime} \gamma_{S B M}^{p_{1}, p_{2}}\left(t^{\prime}\right)= & -g\left(\operatorname { R e } \left[\frac{\pi T}{\sinh \left(\pi T\left(t+1 / \omega_{d}-i / D\right)\right)}\right.\right. \\
& \left.\times \frac{\sinh \left(\pi T\left(2 / \omega_{d}\right)\right)}{\sinh \left(\pi T\left(t-1 / \omega_{d}-i / D\right)\right)}\right] \\
& \left.+i p_{2} \operatorname{Im}\left[\frac{2 / \omega_{d}}{\left(t+1 / \omega_{d}-i / D\right)\left(t-1 / \omega_{d}-i / D\right)}\right]\right)
\end{aligned}
$$

Here, we assumed that the bosons, which are represented by the shifted phonon operators (see Eq. (4.17)), are in equilibrium. Accounting for the original phonons being in equilibrium would give rise to correction terms to the above pair contractions, which are of second order in $g$. In fact, within numerical errors this correction does not change the results for $I_{s t}$. In Eqs. (4.62) and (4.63) one again recognizes that the influence of the off-diagonal electron-phonon interaction strongly depends on the width of the electron densities $\sigma=|\vec{d}| \omega_{d} / D$. The fermionic contractions can be written as

$$
\gamma_{\eta f, \eta^{\prime} f^{\prime}}^{p_{1},+}(t)=\gamma_{\eta^{\prime} f^{\prime}, \eta f}^{p_{1},-}(-t)=\delta_{\eta,-\eta^{\prime}} \delta_{f, f^{\prime}} \gamma_{\eta f}(t)
$$


where the indices $\eta$ and $f$ run over \pm and $L, R$. Using again the definitions in Eqs. (4.35) and (4.36) and the assumption $\Gamma_{f}(\epsilon) \approx$ const. we obtain

$$
\gamma_{\eta f}(t)=\frac{-i T \Gamma_{f} e^{-i \eta \mu_{f} t}}{2 \sinh \left(\pi T\left(t-i / D_{f}\right)\right)} .
$$

Here, we also introduced a bandwidth $D_{f}$ of the reservoir $f$.

To obtain the explicit form of the RG equations one has to make a choice of the $t_{c}$ dependence of the bath contractions $\gamma_{\mu_{1}, \mu_{2}}^{p_{1}, p_{2}}\left(t, t_{c}\right)$. Since the bosonic bath contractions $\gamma_{b_{j}, b_{k}}^{p_{1}, p_{2}}(t)(j, k=1,2,3)$ correspond to those of the spin-boson model, we choose the $t_{c}$ dependence analogously to $\gamma_{2}^{p_{1}, p_{2}}\left(t, t_{c}\right)$ in Section 3.3. Thus, we set

$$
\begin{aligned}
\gamma_{b_{j}, b_{k}}^{p_{1}, p_{2}}\left(t, t_{c}\right)= & \frac{d}{d t}\left(\tilde{R}_{j k}(t) \Theta\left(t-t_{c}\right)\right)+i p_{2} S_{j k}(t) \Theta\left(t-t_{c}\right) \\
+\Theta\left(t-t_{c}\right) \frac{d}{d t} g \pi T \operatorname{sign}(t) & \\
& \times\left\{\begin{array}{cc}
1 & \text { for } j=k=1 \text { or } j=k=3 \\
\frac{1}{2} e^{-D / \omega_{d}} & \text { for } j=k=2 \\
0 & \text { else }
\end{array},\right.
\end{aligned}
$$

where we defined

$$
\begin{aligned}
\tilde{R}_{11}(t)= & g \operatorname{Re}[\pi T \operatorname{coth}(\pi T(t-i / D)) \\
& \left.+\frac{\omega_{d}}{2} \ln \left(\frac{\sinh \left(\pi T\left(t-1 / \omega_{d}-i / D\right)\right)}{\sinh \left(\pi T\left(t+1 / \omega_{d}-i / D\right)\right)}\right)\right] \\
& -g \pi T \operatorname{sign}(t), \\
S_{11}(t)= & g \operatorname{Im}\left[\frac{1 / \omega_{d}^{2}}{\left((t-i / D)^{2}-1 / \omega_{d}^{2}\right)(t-i / D)^{2}}\right], \\
\tilde{R}_{12}= & \tilde{R}_{21}=S_{12}=S_{21}=0, \\
\tilde{R}_{13}= & \tilde{R}_{31}=S_{13}=S_{31}=0,
\end{aligned}
$$




$$
\begin{aligned}
\tilde{R}_{22}(t)= & \frac{g}{2} e^{-D / \omega_{d}} \operatorname{Re}[\pi T \operatorname{coth}(\pi T(t-i / D))] \\
& -\frac{g}{2} e^{-D / \omega_{d}} \pi T \operatorname{sign}(t) \\
S_{22}(t)= & -\frac{g}{2} e^{-D / \omega_{d}} \operatorname{Im}\left[\frac{1}{(t-i / D)^{2}}\right] \\
\tilde{R}_{23}(t)= & \tilde{R}_{32}(t)=-g \operatorname{Re}\left[\omega_{d} e^{-D / 2 \omega_{d}}\right. \\
& \left.\times \ln \left(\frac{\sinh \left(\pi T\left(t-1 / 2 \omega_{d}-i / D\right)\right)}{\sinh \left(\pi T\left(t+1 / 2 \omega_{d}-i / D\right)\right)}\right)\right] \\
S_{23}(t)= & S_{32}(t)=-g \operatorname{Im}\left[\frac{e^{-D / 2 \omega_{d}}}{\left((t-i / D)^{2}-1 / 4 \omega_{d}^{2}\right)}\right] \\
\tilde{R}_{33}(t)= & g \operatorname{Re}[\pi T \operatorname{coth}(\pi T(t-i / D)) \\
& \left.-\frac{\omega_{d}}{2} \ln \left(\frac{\sinh \left(\pi T\left(t-1 / \omega_{d}-i / D\right)\right)}{\sinh \left(\pi T\left(t+1 / \omega_{d}-i / D\right)\right)}\right)\right] \\
& -g \pi T \operatorname{sign}(t) \\
S_{33}(t)= & g \operatorname{Im}\left[\frac{1 / \omega_{d}^{2}-2(t-i / D)^{2}}{\left((t-i / D)^{2}-1 / \omega_{d}^{2}\right)(t-i / D)^{2}}\right]
\end{aligned}
$$

Regarding the fermionic contractions we choose the standard sharp cutoff:

$$
\gamma_{\eta f, \eta^{\prime} f^{\prime}}^{p_{1}, p_{2}}\left(t, t_{c}\right)=\gamma_{\eta f, \eta^{\prime} f^{\prime}}^{p_{1}, p_{2}}(t) \Theta\left(t-t_{c}\right)
$$

Furthermore, because of Eq. (4.66) we can write

$$
\begin{aligned}
\gamma_{\eta f \eta^{\prime} f^{\prime}}^{p_{1} p_{2}}(t)= & \delta_{\eta,-\eta^{\prime}} \delta_{f, f^{\prime}}\left(\frac{1}{2}\left(\gamma_{\eta f}(t)+\gamma_{-\eta f}(-t)\right)\right. \\
& \left.+\frac{p_{2}}{2}\left(\gamma_{\eta f}(t)-\gamma_{-\eta f}(-t)\right)\right)
\end{aligned}
$$

With

$$
\lim _{D_{f} \rightarrow \infty} \frac{1}{2}\left(\gamma_{\eta f}(t)+\gamma_{-\eta f}(-t)\right)=\frac{\Gamma_{f}}{2} \delta(t)
$$

we obtain for large $D_{L}\left(D_{R}\right)$

$$
\gamma_{\eta f, \eta^{\prime} f^{\prime}}^{p_{1}, p_{2}}\left(t, t_{c}\right)=\delta_{\eta,-\eta^{\prime}} \delta_{f, f^{\prime}}\left(\frac{\Gamma_{f}}{2} \delta(t) \Theta\left(t-t_{c}\right)+\bar{\gamma}_{\eta f}^{p_{1}, p_{2}}(t) \Theta\left(t-t_{c}\right)\right)
$$

where we have introduced

$$
\begin{aligned}
\bar{\gamma}_{\eta f}^{p_{1}, p_{2}}(t) & =\frac{p_{2}}{2}\left(\gamma_{\eta f}(t)-\gamma_{-\eta f}(-t)\right) \\
& =-i \frac{p_{2}}{2} T \Gamma_{f} e^{-i \eta \mu_{f} t} \operatorname{Re}\left[\frac{1}{\sinh \left(\pi T\left(t-i / D_{f}\right)\right)}\right]
\end{aligned}
$$

Note that the above choice of the $t_{c}$ dependence of the bath contractions yields a welldefined RG scheme, since $\gamma_{\mu_{1}, \mu_{2}}^{p_{1}, p_{2}}\left(t, t_{c}=0\right)=\gamma_{\mu_{1}, \mu_{2}}^{p_{1}, p_{2}}(t)$. As for the spin-boson model, the 
last term in Eq. (4.68) can be incorporated in the initial conditions. In Section 3.3 we have seen that such terms only contribute to an initial renormalization of $L_{0}$ and $\Sigma_{(I)}$. The first term in Eq. (4.82) will be accounted for in the same way, so that we obtain the modified initial conditions

$$
\begin{aligned}
L_{0}\left(t_{c}=0\right)= & L_{0}-i g \pi T \sum_{p_{1}, p_{2}}\left(G_{b_{1}}^{p_{1}} G_{b_{1}}^{p_{2}}+\frac{1}{2} e^{-D / \omega_{d}} G_{b_{2}}^{p_{1}} G_{b_{2}}^{p_{2}}+G_{b_{3}}^{p_{1}} G_{b_{3}}^{p_{2}}\right) \\
& -i \sum_{\eta f, p_{1}, p_{2}} \frac{\Gamma_{f}}{4} \hat{\sigma}^{p_{1}, p_{2}} G_{\eta f}^{p_{1}} G_{-\eta f}^{p_{2}}, \\
\Sigma\left(z, t_{c}=0\right)= & -g \pi T \sum_{p_{1}, p_{2}}\left(G_{b_{1}}^{p_{1}} G_{b_{1}}^{p_{2}}+\frac{1}{2} e^{-D / \omega_{d}} G_{b_{2}}^{p_{1}} G_{b_{2}}^{p_{2}}+G_{b_{3}}^{p_{1}} G_{b_{3}}^{p_{2}}\right) \\
& -\sum_{\eta f, p_{1}, p_{2}} \frac{\Gamma_{f}}{4} \hat{\sigma}^{p_{1}, p_{2}} G_{\eta f}^{p_{1}} G_{-\eta f}^{p_{2}}, \\
\Sigma_{I}\left(z, t_{c}=0\right)= & -\frac{\Gamma_{L}}{4} \sum_{\eta, p_{1}, p_{2}} \hat{\sigma}^{p_{1}, p_{2}} A_{I \eta L}^{p_{1}} G_{-\eta L}^{p_{2}} .
\end{aligned}
$$

Substituting the remaining terms of Eqs. (4.68) and (4.82) in Eqs. (3.60) - (3.64) and accounting for the additional signs for fermionic contractions yields the final RG equations:

$$
\begin{aligned}
& \frac{d L_{0}}{d t_{c}}= \sum_{p_{1}, p_{2}, j, k}\left(\tilde{R}_{j k}\left(t_{c}\right)\left[G_{b_{j}}^{p_{1}}\left(t_{c}\right), L_{0}\right]+p_{2} S_{j k}\left(t_{c}\right) G_{b_{j}}^{p_{1}}\left(t_{c}\right)\right) G_{b_{k}}^{p_{2}}(0) \\
&-i \sum_{p_{1}, p_{2}, \eta, f} \bar{\gamma}_{\eta f}^{p_{1} p_{2}}\left(t_{c}\right) \hat{\sigma}^{p_{1} p_{2}} G_{\eta f}^{p_{1}}\left(t_{c}\right) G_{-\eta f}^{p_{2}}(0), \\
& \frac{d G_{\mu}^{p}}{d t_{c}}= \sum_{p_{1}, p_{2}, j, k}\left(\tilde{R}_{j k}\left(t_{c}\right)\left(G_{b_{j}}^{p_{1}}\left(t_{c}\right) G_{\mu}^{p}(0)-G_{\mu}^{p}(0) G_{b_{j}}^{p_{1}}\left(t_{c}\right)\right) G_{b_{k}}^{p_{2}}(0)\right. \\
&-i \int_{0}^{t_{c}} d t\left(G_{b_{j}}^{p_{1}}(t) G_{\mu}^{p}(0)-G_{\mu}^{p}(0) G_{b_{j}}^{p_{1}}(t)\right) \\
&\left.\times\left(\tilde{R}_{j k}\left(t_{c}\right)\left[L_{0}, G_{b_{k}}^{p_{2}}\left(t-t_{c}\right)\right]+p_{2} S_{j k}\left(t_{c}\right) G_{b_{k}}^{p_{2}}\left(t-t_{c}\right)\right)\right) \\
&+\sum_{p_{1}, p_{2}, \eta, f} \bar{\gamma}_{\eta f}^{p_{1} p_{2}}\left(t_{c}\right) \int_{0}^{t_{c}} d t\left(G_{\mu}^{p}(0) \hat{\sigma}^{p_{1} p_{2}} G_{\eta f}^{p_{1}}(t)\right. \\
&\left.-\eta_{\mu}^{p p_{2}} \hat{\sigma}^{p_{1} p_{2}} G_{\eta f}^{p_{1}}(t) G_{\mu}^{p}(0)\right) G_{-\eta f}^{p_{2}}\left(t-t_{c}\right),
\end{aligned}
$$




$$
\begin{aligned}
& \frac{d A_{(I) \mu}^{p}}{d t_{c}}(z)=\sum_{p_{1}, p_{2}, j, k}\left(\tilde{R}_{j k}\left(t_{c}\right)\left(A_{(I) b_{j}}^{p_{1}}\left(t_{c}, z\right) G_{\mu}^{p}(0)-A_{(I) \mu}^{p}(0, z) G_{b_{j}}^{p_{1}}\left(t_{c}\right)\right) G_{b_{k}}^{p_{2}}(0)\right. \\
& -i \int_{0}^{t_{c}} d t\left(A_{(I) b_{j}}^{p_{1}}(t, z) G_{\mu}^{p}(0)-A_{(I) \mu}^{p}(0, z) G_{b_{j}}^{p_{1}}(t)\right) \\
& \left.\times\left(\tilde{R}_{j k}\left(t_{c}\right)\left[L_{0}, G_{b_{k}}^{p_{2}}\left(t-t_{c}\right)\right]+p_{2} S_{j k}\left(t_{c}\right) G_{b_{k}}^{p_{2}}\left(t-t_{c}\right)\right)\right) \\
& +\sum_{p_{1}, p_{2}, \eta, f} \bar{\gamma}_{\eta f}^{p_{1} p_{2}}\left(t_{c}\right) \int_{0}^{t_{c}} d t\left(A_{(I) \mu}^{p}(0, z) \hat{\sigma}^{p_{1} p_{2}} G_{\eta f}^{p_{1}}(t)\right. \\
& \left.-\eta_{\mu}^{p p_{2}} \hat{\sigma}^{p_{1} p_{2}} A_{(I) \eta f}^{p_{1}}(t, z) G_{\mu}^{p}(0)\right) G_{-\eta f}^{p_{2}}\left(t-t_{c}\right), \\
& \frac{d B_{\mu}^{p}}{d t_{c}}(z)=\sum_{p_{1}, p_{2}, j, k}\left(\tilde{R}_{j k}\left(t_{c}\right) G_{b_{j}}^{p_{1}}\left(t_{c}\right) G_{\mu}^{p}(0) B_{b_{k}}^{p_{2}}(0, z)\right. \\
& -i \int_{0}^{t_{c}} d t G_{b_{j}}^{p_{1}}(t) G_{\mu}^{p}(0) \\
& \left.\times\left(\tilde{R}_{j k}\left(t_{c}\right)\left(L_{0}-z\right)+p_{2} S_{j k}\left(t_{c}\right)\right) B_{b_{k}}^{p_{2}}\left(t-t_{c}, z\right)\right) \\
& -\sum_{p_{1}, p_{2}, \eta, f} \bar{\gamma}_{\eta f}^{p_{1} p_{2}}\left(t_{c}\right) \int_{0}^{t_{c}} d t \eta_{\mu}^{p_{2}} \hat{\sigma}^{p_{1} p_{2}} G_{\eta f}^{p_{1}}(t) G_{\mu}^{p}(0) B_{-\eta f}^{p_{2}}\left(t-t_{c}, z\right), \\
& \frac{d \Sigma_{(I)}}{d t_{c}}(z)=-i \sum_{p_{1}, p_{2}, j, k} A_{(I) b_{j}}^{p_{1}}\left(t_{c}, z\right)\left(\tilde{R}_{j k}\left(t_{c}\right)\left(L_{0}-z\right)+p_{2} S_{j k}\left(t_{c}\right)\right) B_{b_{k}}^{p_{2}}(0, z) \\
& -\sum_{p_{1}, p_{2}, \eta, f} \bar{\gamma}_{\eta f}^{p_{1} p_{2}}\left(t_{c}\right) \hat{\sigma}^{p_{1} p_{2}} A_{(I) \eta f}^{p_{1}}\left(t_{c}, z\right) B_{-\eta f}^{p_{2}}(0, z) .
\end{aligned}
$$

Note that the convergence condition, Eq. (3.39), is fulfilled, since

$$
\begin{aligned}
\lim _{t \rightarrow \infty} \tilde{R}_{b_{j} b_{k}}(t) & =\lim _{t \rightarrow \infty} S_{b_{j} b_{k}}(t) \\
& =\lim _{t \rightarrow \infty} \bar{\gamma}_{\eta f}^{p_{1}, p_{2}}(t)=0 .
\end{aligned}
$$

The generation of multiple vertex superoperators is neglected here as in Chapter 3. Within this approximation we solved the spin-boson model for couplings up to $\alpha=g / 2 \lesssim 0.1-0.2$. Since realistic values of $g$ are significantly smaller $(g=0.05$ for GaAs), our approach will lead to very reliable results. Furthermore, in contrast to the spin-boson model we do not introduce a crossover between different definitions of the $t_{c}$ dependent bosonic bath contractions (see Section 3.3), since in the relevant coupling regime the results turn out to be independent of such a crossover. Note that by using the RTRG for the spin-boson model we achieved reliable results for arbitrary bias $\epsilon$ and temperature $T$, and we also obtained the off-diagonal elements of the reduced density matrix. That means for the coupled quantum dots, that the solution of the above RG equations will yield accurate results for the stationary current $I_{s t}$ for arbitrary $\epsilon$ even at low temperature, whereas an approach using the NIBA can give $I_{s t}$ as a function of $\epsilon$ only for sufficiently high temperature, see Section 4.1. 
Since also the coupling to the leads is treated nonperturbatively, the induced level broadening is accounted for properly, so that we do not have to simulate that by an additional cutoff parameter. Furthermore, a finite value of the external voltage $V$ is included in our method, so that we obtain correct results also in the regime $\epsilon \approx V$. Eventually, using the RTRG we are also able to account for the off-diagonal electron-phonon interaction, which is important for small $D / \omega_{d}$, see Eqs. (4.23), (4.62) and (4.63).

\subsection{Results}

We solve the set of ordinary differential equations, Eqs. (4.87) - (4.91), numerically, where we again use the methods described in Appendix E.1 and E.2. The stationary tunnel current $I_{s t}$ then follows from Eqs. (4.54) and (4.55). Our choice of the parameters corresponds to the experiment, where a GaAs structure was used at the temperature $T=23 m K=1.98 \mu \mathrm{eV}$ with an external voltage $V=140 \mu \mathrm{eV}$ [30]. For GaAs we deal with $c_{s}=5000 \mathrm{~m} / \mathrm{s}$ and $g=0.05$ [82]. The distance between the dots is estimated as $d=200 \cdot 10^{-9} \mathrm{~m}$ [34], which yields $\omega_{d}=16.5 \mu \mathrm{eV}$.

Let us first study the parameters $T_{c}=\Gamma_{L}=\Gamma_{R}=1 \mu \mathrm{eV}, D_{L}=D_{R}=1 \mathrm{meV}$ and $D=150 \mu \mathrm{eV}$, which corresponds to the parameters studied in Ref. [34]. The $t_{c}$ flow of $I_{s t}$ for different energy differences $\epsilon$ is shown in Fig. 4.5. The external voltage $V$ gives rise to a finite stationary tunnel current, which we see in the limit of $t_{c} \rightarrow \infty$.

The asymptotic $\left(t_{c} \rightarrow \infty\right)$ result for $I_{s t}$ as a function of $\epsilon$ for the same parameters, but also for $D=100 \mu \mathrm{eV}$ is shown in Fig. 4.6. The elastic current can be seen around $\epsilon$ with a width depending on the coupling to the leads $\Gamma_{L}=\Gamma_{R}$ and the internal tunnel amplitude $T_{c}$ (see Eq. (4.37)). There the phonons do not participate in the tunnel process. Due to the coupling to the phonons there is also an inelastic current, where phonons are emitted $(\epsilon>0)$ respectively absorbed $(\epsilon<0)$ during the tunnel process. For increasing $\Gamma_{L(R)}$ the width of the elastic current grows, while an increased coupling constant $g$ leads to a larger inelastic current. The effect of the finite voltage $V$ can be seen in Fig. 4.6, where for $\epsilon>V$ the tunnel current drops to zero. Furthermore, Fig. 4.6 shows that the off-diagonal interaction leads to a larger inelastic current. This effect is increased with decreasing $D$, see also Eqs. (4.62) - (4.63). We will see below (Fig. 4.8) that, due to Eq. (4.23), the off-diagonal interaction also suppresses the elastic current. Eventually, in Fig. 4.6 one also recognizes the oscillations stemming from the interference of the phonons interacting with the two dots [34].

Let us now study the current quantitatively in comparison with the experiment. For this it is necessary to choose realistic parameter values for $T_{c}, \Gamma_{L(R)}, D$ and $D_{L(R)}$. From changing the bias polarity in the experiments the ratio $\Gamma_{R} / \Gamma_{L} \approx 0.5 . .1$ was found [30]. To determine the couplings $T_{c}$ and $\Gamma_{R}$, the experimental data are compared with the result of Stoof and Nazarov, given in Eq. (4.37), which is valid for no electron-phonon interaction $(g=0)$. A good agreement of the elastic current is found for $T_{c}=0.124 \mu \mathrm{eV}$ and $\Gamma_{L}=\Gamma_{R}=3.5 \mu \mathrm{eV}$, see Fig. 4.7. However, due to the absent electron-phonon interaction the influence of the off-diagonal interaction terms on the internal tunnel amplitude $T_{c}^{\text {eff }}$ is 


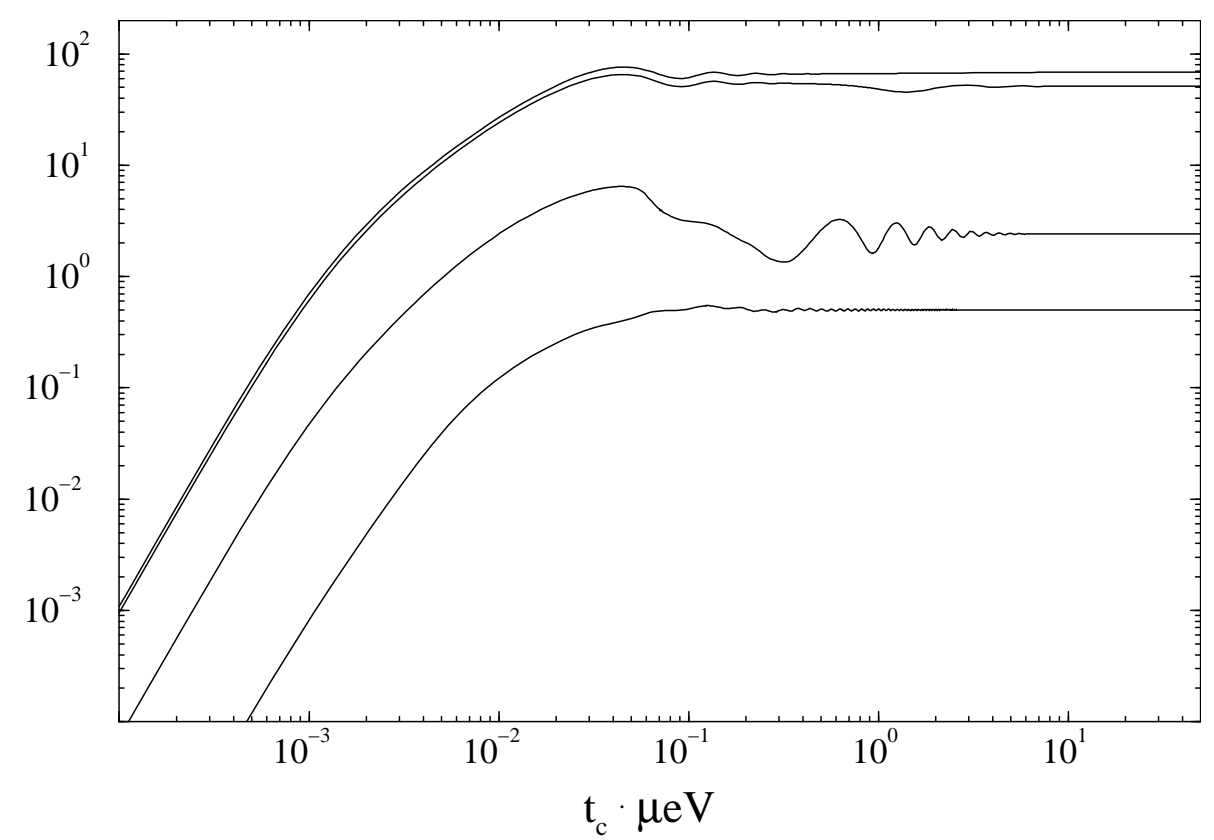

Figure 4.5: Stationary tunnel current $I_{s t}$ as a function of $t_{c}$ for $T_{c}=\Gamma_{L}=\Gamma_{R}=1 \mu \mathrm{eV}$, $D=150 \mu \mathrm{eV}, D_{L}=D_{R}=1 \mathrm{meV}$ and $\epsilon / \mu \mathrm{eV}=0,1,10,100$ (from top to bottom).

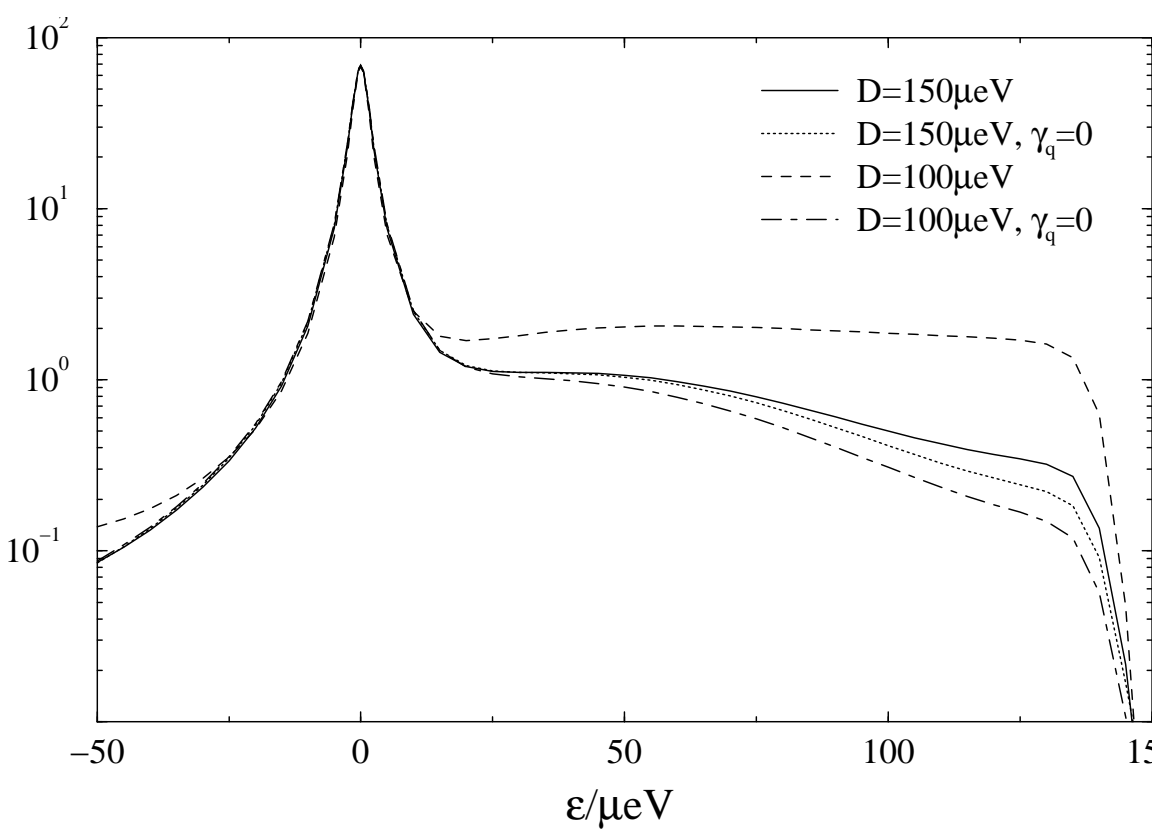

Figure 4.6: Stationary tunnel current $I_{s t}$ as a function of the energy difference $\epsilon$ for $T_{c}=$ $\Gamma_{L}=\Gamma_{R}=1 \mu \mathrm{eV}$ and $D_{L}=D_{R}=1 \mathrm{meV}$. Solid line: $D=150 \mu \mathrm{eV}$. Dotted line: $D=150 \mu \mathrm{eV}, \gamma_{q}=0$. Dashed line: $D=100 \mu \mathrm{eV}$. Dot-dashed line: $D=100 \mu \mathrm{eV}$, $\gamma_{q}=0$ 


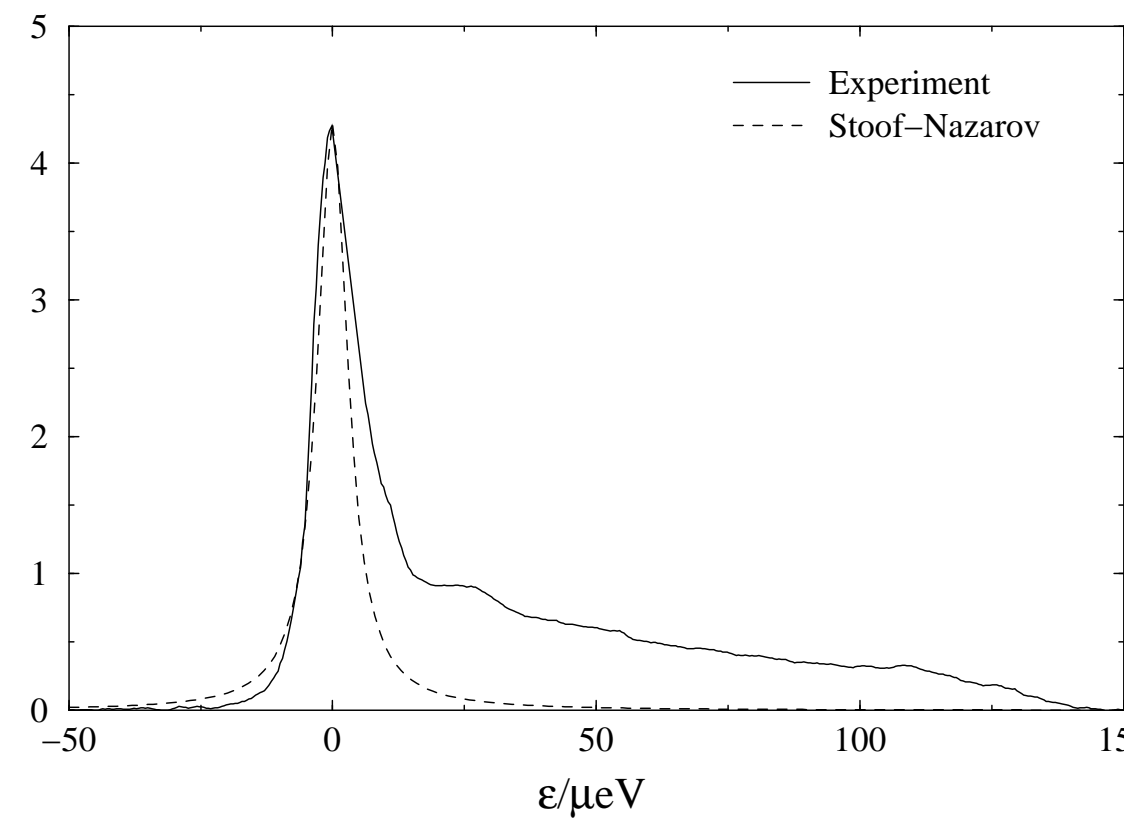

Figure 4.7: Stationary tunnel current $I_{s t}$ as a function of the energy difference $\epsilon$. Solid line: Experiment [30]. Dashed line: Stoof-Nazarov result for the case of no electronphonon interaction, with the parameters $T_{c}=0.124 \mu \mathrm{eV}$ and $\Gamma_{L}=\Gamma_{R}=3.5 \mu \mathrm{eV}$.

disregarded in the Stoof-Nazarov result. In contrast, within our approach this influence is accounted for in Eq. (4.23). In case of a finite extension of the electron densities within the dots, i.e. finite ratios $D / \omega_{d}$, the tunnel amplitude is effectively reduced. Therefore, the Stoof-Nazarov result underestimates the value of $T_{c}$.

From the large inelastic current in the experiment one can conclude, that in fact a finite value of $D$ was realized. It turns out that $T_{c} \approx 0.375$ allows sensible fits. First, in Fig. 4.8 our results for $T_{c}=0.375 \mu \mathrm{eV}, \Gamma_{L}=\Gamma_{R}=3.5 \mu \mathrm{eV}, D_{L}=D_{R}=1 \mathrm{meV}$ and $D=70 \mu \mathrm{eV}$ respectively $D=100 \mu \mathrm{eV}$ are compared with the experiment. One recognizes that with decreasing $D$ the larger overlap of the dots' wavefunctions leads to a stronger impact of the off-diagonal electron-phonon interaction. The elastic current is suppressed, whereas the inelastic current is increased. The deviations from the experiment show, that there is an $\epsilon$ dependence of the width of the electron densities, which we have to account for in order to achieve agreement. In Fig. 4.9 we show a fit of the width $\sigma=d \omega_{d} / D$, which is based on the experimental results for $I_{s t}$. One recognizes that for larger absolute values of $\epsilon$ the electron densities are more sharply peaked. The asymmetry is due to the finite external voltage $V$. For $\epsilon<0$ the state $|L\rangle$ lies in a deep potential well, thus this energetic separation of the two quantum dot levels and the leads causes a very small overlap of the wavefunctions within the dots. On the other hand for $\epsilon>0$ neither dot level lies in a deep potential well, however, an increasing energetic separation $\epsilon$ again leads to more sharply defined electron densities.

Eventually, in Fig. 4.8 one also recognizes that the structure on the emission side 


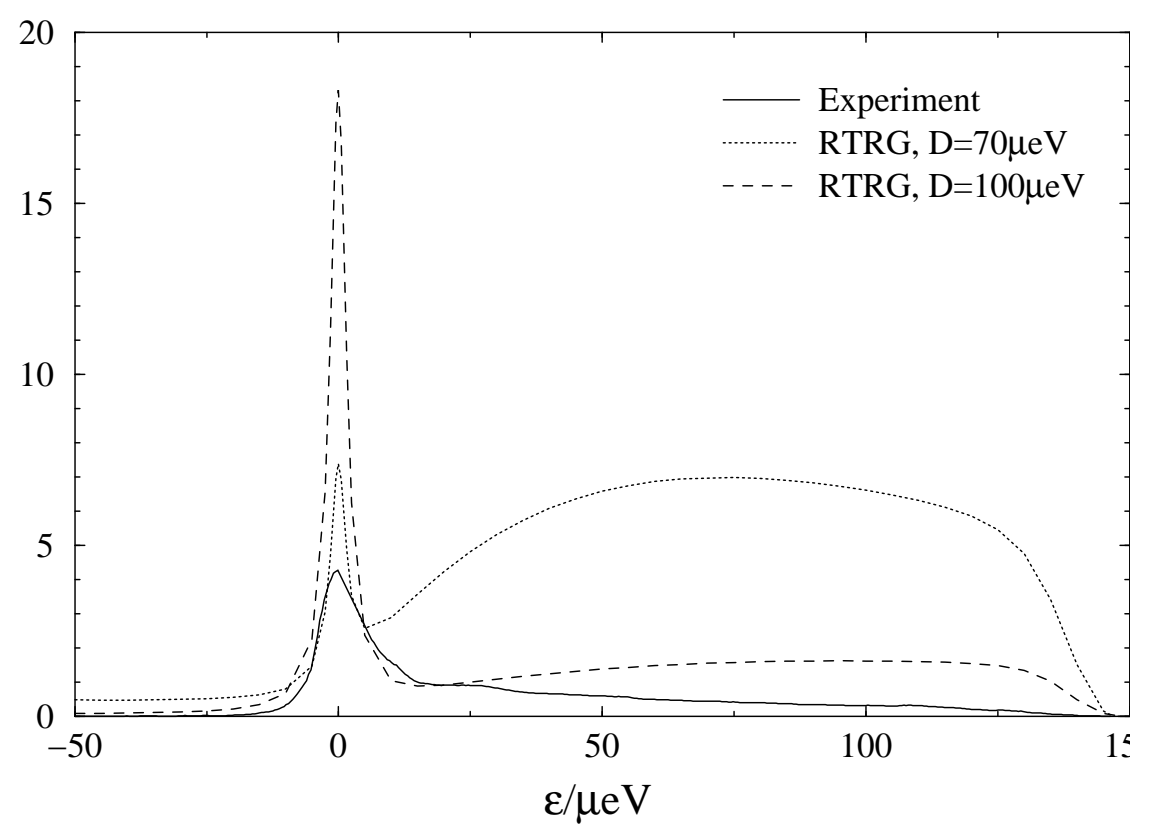

Figure 4.8: Stationary tunnel current $I_{s t}$ as a function of the energy difference $\epsilon$. Solid line: Experiment [30]. Dotted line: RTRG with $T_{c}=0.375 \mu \mathrm{eV}, \Gamma_{L}=\Gamma_{R}=3.5 \mu \mathrm{eV}$, $D_{L}=D_{R}=1 \mathrm{meV}$ and $D=70 \mu \mathrm{eV}$. Dashed line: RTRG with $T_{c}=0.375 \mu \mathrm{eV}$, $\Gamma_{L}=\Gamma_{R}=3.5 \mu \mathrm{eV}, D_{L}=D_{R}=1 \mathrm{meV}$ and $D=100 \mu \mathrm{eV}$.

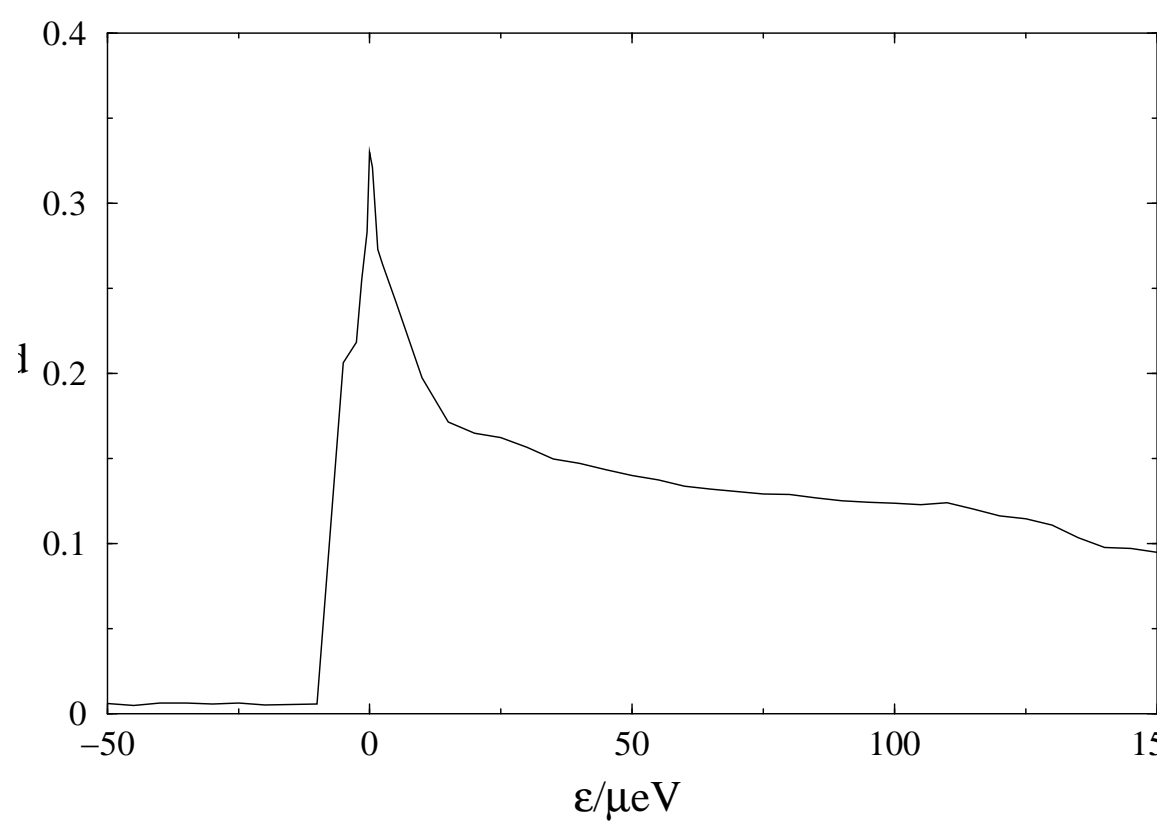

Figure 4.9: The width of the electron density within one dot, $\sigma$, as a function of the energy difference $\epsilon . T_{c}=0.375 \mu \mathrm{eV}, \Gamma_{L}=\Gamma_{R}=3.5 \mu \mathrm{eV}$ and $D_{L}=D_{R}=1 \mathrm{meV}$. 
$(\epsilon>0)$ of the current spectrum observed in the experiment (see also Fig. 4.7) does not stem from interference effects of the phonons. In fact, the oscillations generated by this interference occur on a much larger energy scale than the structure found in the experimental curve. In contrast, our results show that the bump on the emission side of the current spectrum (Fig. 4.7) is due to another mechanism: on the one hand we see in Fig. 4.8 that for small $\epsilon>0$ the inelastic current grows with increasing $\epsilon$. On the other hand however, the electron densities are simultaneously sharpened, so that the cutoff $D$ is increased. For larger $\epsilon$ this again reduces the inelastic current.

\subsection{Summary}

A coupled quantum dot system in a phonon bath in nonequilibrium was examined. We have explained the close relation of this problem to the previously studied spin-boson model. As in case of the spin-boson model we applied the RTRG to this problem and determined the stationary tunnel current. In this case it was necessary to formulate the RTRG also for observables, which are linear in the bath field operators, and we had to account for additional signs arising from the commutation of fermionic field operators.

By accounting for both the coupling to the leads and the coupling to the environmental phonons nonperturbatively we achieved a reliable solution for the stationary tunnel current in the whole parameter space with moderately large couplings. Especially, we obtained accurate results for arbitrary energy difference $\epsilon$ between the dots and temperature $T$, which is in contrast to approximations using the NIBA. Furthermore, we included off-diagonal terms of the electron-phonon interaction, which give a contribution in case of finite extensions of the electron densities within the dots. Thereby, for the first time both the elastic and the inelastic current of the experiment could quantitatively be reproduced [35]. Our analysis shows the importance of the finite width $\sigma$ of the electron densities within one dot, and for the experiment, the dependence of $\sigma$ on $\epsilon$ was calculated.

For the coupling parameters realized in the experiment [30] the neglecting of double and higher-order vertex objects was justified. In contrast, in the next chapter we will study a Kondo model, for which we will have to account for double vertices. We have already mentioned in Section 3.2, that we will use a modified formulation of the RTRG to treat these double vertex objects. 


\section{Chapter 5}

\section{Two-lead Kondo model}

The Kondo model describes a magnetic impurity coupled to a band of conduction electrons. Over the past decades this problem has often been studied both experimentally and theoretically (for a review see Ref. [36]). When Kondo first studied the model perturbatively in 1964 [83], he found low-temperature divergencies. In fact, perturbative studies give only a good description of the problem for $T \gg T_{K}$, where $T_{K}$ is the Kondo temperature. Renormalization-group studies show that the coupling constant increases when reducing the relevant energy scale. Thus, perturbative approaches like Poor Man's scaling break down at $T_{K}$. In 1975, the problem was solved by Wilson [5] by applying the numerical renormalization-group method to the Kondo model. Later, a solution was also found by using the Bethe ansatz $[84,85]$, an approach which had first been introduced in Ref. [86].

In recent experiments quantum dot systems served as realizations of the Kondo model $[87,88,89,77]$. Such systems have the advantage that the parameters can be tuned within a wide range. When one applies an external bias voltage to the quantum dot, one deals with a nonequilibrium problem, the two-lead Kondo model (TLKM). It has attracted much theoretical interest and the question has been raised, if an external voltage induces twochannel physics [37] - [43].

We apply the RTRG method to the problem. As the bare Hamiltonian already involves double vertices, the usual formulation would lead to complicated equations because of retardation effects (see Appendix C). Therefore, we use a formulation in energy space, by which we avoid the problem of retardation. However, one then has to account for an additional frequency-dependence of the vertices. Furthermore, this method naturally leads to divergencies, if the cutoff is defined only with respect to the frequency of the contraction vertices. We introduce a generalized definition of the cutoff-function, which also depends on the external vertices. This new scheme allows a RG study of the coupling constants of the two-lead Kondo model. We study an effective Hamiltonian for vanishing temperature $T$. Thus, effects which are only taken into account by an analysis on the Keldysh contour, such as rates, are neglected. Thereby we quantitatively find a two-channel behaviour for the running couplings which, on this level, was previously proposed in a qualitative analysis [37]. The influence of rates, which arise for a finite bias, is discussed. 

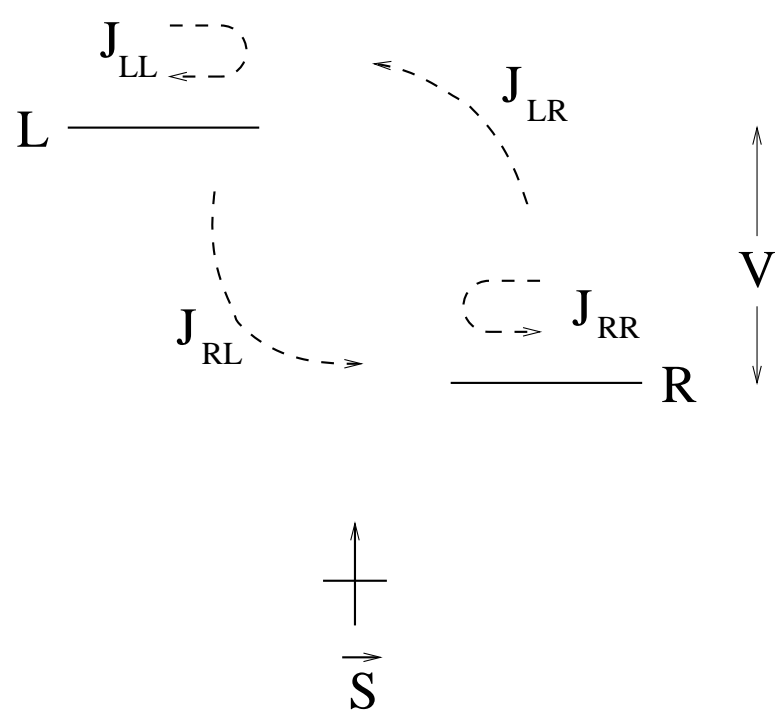

Figure 5.1: The two-lead Kondo model. The voltage $V$ is applied between two leads ( $L$ and $R$ ), which are coupled to the impurity spin $\vec{S}$. The coupling constants $J_{L L}$ and $J_{R R}$ ( $J_{L R}$ and $J_{R L}$ ) correspond to reflection (transmission) of conduction electrons.

\subsection{Model Hamiltonian}

In 1964 Kondo studied a model Hamiltonian for a magnetic impurity in a conduction band [83]. There an exchange scattering potential gives rise to an interaction part of the Hamiltonian $H_{V}$, which is parametrized by the energy $J$ :

$$
H_{V}=\sum_{k \sigma \sigma^{\prime}} J c_{k \sigma}^{\dagger} \vec{\sigma}_{\sigma \sigma^{\prime}} c_{k \sigma^{\prime}} \vec{S}
$$

Here, $\vec{S}$ is the spin of the impurity and $\vec{\sigma}$ is the vector consisting of the Pauli spin matrices. $c_{k \sigma}^{\dagger}\left(c_{k \sigma}\right)$ creates (annihilates) an electron in the conduction band with the energy $\epsilon_{k}$ and the spin $\sigma$. The total Hamiltonian can be written as a sum $H=H_{B}+H_{V}$, where $H_{B}$ denotes the free electron part for the conduction electrons. When two bands are coupled to the impurity, we end up with

$$
\begin{aligned}
& H_{B}=\sum_{\alpha k \sigma} \epsilon_{\alpha k} c_{\alpha k \sigma}^{\dagger} c_{\alpha k \sigma} \\
& H_{V}=\sum_{\alpha \alpha^{\prime} k \sigma \sigma^{\prime}} J_{\alpha \alpha^{\prime}} c_{\alpha k \sigma}^{\dagger} \vec{\sigma}_{\sigma \sigma^{\prime}} c_{\alpha^{\prime} k \sigma^{\prime}} \vec{S}
\end{aligned}
$$

where the index $\alpha=L(R)$ refers to the left (right) electron reservoir. The coupling constants $J_{L L}$ and $J_{R R}$ correspond to a reflection of conduction electrons, whereas $J_{L R}$ and $J_{R L}$ give rise to transmission from one reservoir to the other. Between them a finite external voltage $V$ may be applied (see Fig. 5.1).

The case of vanishing bias involves a well-known renormalization-group flow [5, 36]. Introducing the high-energy cutoff $\omega_{c}$ as a flow parameter the antiferromagnetic coupling 
constants increase, when $\omega_{c}$ is successively reduced. This leads to a low-temperature regime where the impurity spin is quenched by the band electrons. The physics is then determined by the only relevant energy scale, the Kondo temperature $T_{K}$. Regarding the above Hamiltonian we use the density of states of the reservoir $\alpha, \rho_{\alpha}$, to define the dimensionless coupling $\bar{J}_{\alpha \alpha^{\prime}}:=\rho_{\alpha} J_{\alpha \alpha^{\prime}}$. In the following we consider the symmetric case, where $\bar{J}:=\bar{J}_{L L}=\bar{J}_{R R}=\bar{J}_{L R}=\bar{J}_{R L}$. The Kondo temperature is given by

$$
T_{K}=D \sqrt{2 \bar{J}} e^{-1 / 4 \bar{J}}
$$

where $D \gg T_{K}$ is the bandwidth of the electron reservoirs. Note that, in this chapter we again use $\hbar=k_{B}=e=1$. For the case $V=0$ the increase of the coupling constant for $T_{K}>T$ follows from the $\mathrm{RG}$ equation $[5,36]$

$$
\begin{aligned}
\frac{d \bar{J}}{d \ln \omega_{c}} & =-4 \bar{J}^{2} \\
\Rightarrow \frac{d \bar{J}^{-1}}{d \ln \omega_{c}} & =4 .
\end{aligned}
$$

For the case of finite $V$, Coleman et al. [37] proposed for the running couplings the existence of two different regimes, which are separated by the energy scale $V$ :

$$
\left.\begin{array}{rlrl}
\frac{d \bar{J}}{d \ln \omega_{c}} & =-4 \bar{J}^{2} & \text { for } \quad \omega_{c} \gg V>T_{K}>T, \\
\frac{d \bar{J}_{L L}}{d \ln \omega_{c}} & =\frac{d \bar{J}_{R R}}{d \ln \omega_{c}}=-2 \bar{J}^{2}, \\
\frac{d \bar{J}_{L R}}{d \ln \omega_{c}} & =\frac{d \bar{J}_{R L}}{d \ln \omega_{c}}=0
\end{array}\right\} \quad \text { for } \quad V \gg \omega_{c} \gg T_{K}>T .
$$

However, since Coleman et al. used a qualitative argumentation within a Poor Man's scaling approach, these equations have not been derived quantitatively. Furthermore, the above equations do not reflect the effects of rates which arise for a finite voltage.

\subsection{The RTRG for the two-lead Kondo model}

We apply the real-time renormalization-group method to obtain the running couplings for $T=0$ and finite bias $V$. As in case of the polaron, we consider the $S$ matrix

$$
\begin{aligned}
S & =\lim _{t_{0} \rightarrow \infty} T e^{-i \int_{-t_{0}}^{t} d t H} \\
& =\lim _{t_{0} \rightarrow \infty} e^{-i H_{B} t} T e^{-i \int_{-t_{0}}^{t} d t^{\prime} H_{V}\left(t^{\prime}\right)} e^{-i H_{B} t_{0}},
\end{aligned}
$$

where $T$ denotes time ordering and $H_{V}$ is taken in the interaction picture with respect to $H_{B}$. The underlying picture is that we turn on the interaction adiabatically in a large time interval, but keep the external voltage at a constant value during that process. Using 
the RTRG we obtain an effective Hamiltonian, which contains the running couplings. However, this approach does not account for the rates generated by the current. To include them one would have to study the problem on the Keldysh contour.

Let us first write the Hamiltonian in a convenient form. We define

$$
\begin{aligned}
g_{\eta \alpha \sigma, \eta^{\prime} \alpha^{\prime} \sigma^{\prime}} & :=\delta_{\eta,-} \delta_{\eta^{\prime},+} J_{\alpha \alpha^{\prime}} \vec{\sigma}_{\sigma \sigma^{\prime}} \vec{S}, \\
j_{+\alpha \sigma} & :=\sum_{k} c_{\alpha k \sigma}, \\
j_{-\alpha \sigma} & :=\sum_{k} c_{\alpha k \sigma}^{\dagger},
\end{aligned}
$$

where the index $\eta$ takes the values \pm referring to out- and ingoing electrons in the corresponding reservoir. With the superindex $\nu=(\eta, \alpha, \sigma)$ we then obtain for $H_{V}$

$$
H_{V}=\sum_{\nu, \nu^{\prime}} \frac{g_{\nu, \nu^{\prime}}-g_{\nu^{\prime}, \nu}}{2}: j_{\nu} j_{\nu^{\prime}}:
$$

Here, : $:$ denotes normal ordering according to Wick's theorem. Note, that

$$
\sum_{\nu, \nu^{\prime}} g_{\nu, \nu^{\prime}}\left\langle j_{\nu} j_{\nu^{\prime}}\right\rangle=0
$$

holds. One obtains for a bath contraction

$$
\begin{aligned}
\left\langle j_{\nu}(t) j_{\nu^{\prime}}\right\rangle & =\delta_{\eta,-\eta^{\prime}} \delta_{\alpha, \alpha^{\prime}} \delta_{\sigma, \sigma^{\prime}} e^{-i \eta \mu_{\alpha} t} \int d \omega e^{-i \eta \omega t} \frac{\rho_{\alpha}}{2} e^{-\eta \omega / D} \Theta(\eta \omega) \\
& =\delta_{\eta,-\eta^{\prime}} \delta_{\alpha, \alpha^{\prime}} \delta_{\sigma, \sigma^{\prime}}(-i) \frac{\rho_{\alpha}}{2} \frac{e^{-i \eta \mu_{\alpha} t}}{t-i / D}
\end{aligned}
$$

where $\mu_{\alpha}$ is the chemical potential of the reservoir $\alpha$ and we assumed a constant density of states $\rho_{\alpha}(\epsilon)=2 \sum_{k} \delta\left(\epsilon-\epsilon_{\alpha k}\right) \approx$ const.. We now proceed analogously to Section 2.3, i.e. we apply Wick's theorem and integrate out certain diagrams. However, in this case the "cross contractions" (see the derivation of the RTRG in Section 3.2) will be specified in energy space. The diagrams giving rise to "cross contractions" may be read from the equation

$$
\begin{aligned}
: j_{\nu_{1}} j_{\nu_{2}}:: j_{\nu_{3}} j_{\nu_{4}}:= & : j_{\nu_{1}} j_{\nu_{2}} j_{\nu_{3}} j_{\nu_{4}}: \\
& +: j_{\nu_{2}} j_{\nu_{3}}:\left\langle j_{\nu_{1}} j_{\nu_{4}}\right\rangle-: j_{\nu_{1}} j_{\nu_{3}}:\left\langle j_{\nu_{2}} j_{\nu_{4}}\right\rangle \\
& +: j_{\nu_{1}} j_{\nu_{4}}:\left\langle j_{\nu_{2}} j_{\nu_{3}}\right\rangle-: j_{\nu_{2}} j_{\nu_{4}}:\left\langle j_{\nu_{1}} j_{\nu_{3}}\right\rangle \\
& +\left\langle j_{\nu_{2}} j_{\nu_{3}}\right\rangle\left\langle j_{\nu_{1}} j_{\nu_{4}}\right\rangle-\left\langle j_{\nu_{1}} j_{\nu_{3}}\right\rangle\left\langle j_{\nu_{2}} j_{\nu_{4}}\right\rangle
\end{aligned}
$$

As we outlined in Appendix C, double vertices lead to retardation effects in the RTRG, thereby causing integro-differential equations. We now develop a formulation in energy space, which avoids this problem. We write for the bath contraction

$$
\left\langle j_{\nu}(t) j_{\nu^{\prime}}\left(t^{\prime}\right)\right\rangle=\delta_{\eta,-\eta^{\prime}} \delta_{\alpha, \alpha^{\prime}} \delta_{\sigma, \sigma^{\prime}} \int d \omega \int d \omega^{\prime} e^{-i \eta \omega t} e^{-i \eta^{\prime} \omega^{\prime} t^{\prime}} \hat{\gamma}_{\nu}\left(\omega, \omega^{\prime}\right)
$$


with

$$
\hat{\gamma}_{\nu}\left(\omega, \omega^{\prime}\right)=\delta\left(\omega-\omega^{\prime}\right) \frac{\rho_{\alpha}}{2} e^{-\eta\left(\omega-\mu_{\alpha}\right) / D} \Theta\left(\eta\left(\omega-\mu_{\alpha}\right)\right) .
$$

The factor $\exp \left(-i\left(\eta \omega t+\eta^{\prime} \omega^{\prime} t^{\prime}\right)\right)$ in Eq. (5.15) may formally be included in the definition of the interaction picture for $g_{\nu, \nu^{\prime}}$ :

$$
g_{\nu, \nu^{\prime}, \omega, \omega^{\prime}}(t)=e^{i H_{0} t} g_{\nu, \nu^{\prime}, \omega, \omega^{\prime}} e^{-i H_{0} t} e^{-i \eta \omega t} e^{-i \eta^{\prime} \omega^{\prime} t} .
$$

$H_{0}$ is the Hamiltonian for the impurity and vanishes initially. Furthermore, we have already accounted for a $\omega$ dependence of the operators $g_{\nu, \nu^{\prime}}$, which is generated during the RG flow. Initially, we start with $g_{\nu, \nu^{\prime}, \omega, \omega^{\prime}}=g_{\nu, \nu^{\prime}}$. By the definition in Eq. (5.17) the information about the time arguments of the bath contractions is contained in $g_{\nu, \nu^{\prime}, \omega, \omega^{\prime}}(t)$, i.e. the vertices may be shifted, so that an additional time-dependence of $g_{\nu, \nu^{\prime}, \omega, \omega^{\prime}}(t)$ is not necessary. The RG is now set up by including the cutoff-function $q\left(\omega, \omega_{c}\right)$. We define

$$
\gamma_{\nu}\left(\omega, \omega_{c}\right)=\frac{\rho_{\alpha}}{2} e^{-\eta\left(\omega-\mu_{\alpha}\right) / D} \Theta\left(\eta\left(\omega-\mu_{\alpha}\right)\right) q\left(\omega-\mu_{\alpha}, \omega_{c}\right),
$$

which is analogous to the corresponding definition in time space (see Eq. (3.36)). Thus, we end up with the RG equations

$$
\begin{aligned}
\frac{d H_{0}}{d \omega_{c}}= & i \int_{0}^{\infty} d t \int d \omega \int d \omega^{\prime} \sum_{\nu, \nu^{\prime}} \frac{d}{d \omega_{c}}\left(\gamma_{\nu}\left(\omega, \omega_{c}\right) \gamma_{\nu^{\prime}}\left(\omega^{\prime}, \omega_{c}\right)\right) \\
& \times\left(g_{\nu^{\prime}, \nu, \omega^{\prime}, \omega}(t / 2)-g_{\nu, \nu^{\prime}, \omega, \omega^{\prime}}(t / 2)\right) g_{-\nu,-\nu^{\prime}, \omega, \omega^{\prime}}(-t / 2), \\
\frac{d g_{\nu_{1}, \nu_{2}, \omega_{1}, \omega_{2}}}{d \omega_{c}}= & i \int_{0}^{\infty} d t \int d \omega \sum_{\nu} \frac{d}{d \omega_{c}} \gamma_{\nu}\left(\omega, \omega_{c}\right) \\
& \times\left(g_{\nu_{1}, \nu, \omega_{1}, \omega}(t / 2)-g_{\nu, \nu_{1}, \omega, \omega_{1}}(t / 2)\right) \\
& \times\left(g_{-\nu, \nu_{2}, \omega, \omega_{2}}(-t / 2)-g_{\nu_{2},-\nu, \omega_{2}, \omega}(-t / 2)\right)
\end{aligned}
$$

where we have used $-\nu=(-\eta, \alpha, \sigma)$. The following ansatz solves the above equations:

$$
\begin{aligned}
H_{0}\left(\omega_{c}\right)= & h\left(\omega_{c}\right) \mathbb{1}, \\
\frac{g_{\nu_{1}, \nu_{2}, \omega_{1}, \omega_{2}}\left(\omega_{c}\right)-g_{\nu_{2}, \nu_{1}, \omega_{2}, \omega_{1}}\left(\omega_{c}\right)}{2}= & J_{\alpha_{1} \alpha_{2}, \omega_{1}, \omega_{2}}\left(\omega_{c}\right)\left(\vec{\sigma}_{\sigma_{1} \sigma_{2}} \vec{S}\right) \\
& +K_{\alpha_{1} \alpha_{2}, \omega_{1}, \omega_{2}}\left(\omega_{c}\right)\left(\delta_{\sigma_{1} \sigma_{2}} \mathbb{1}\right) .
\end{aligned}
$$

The initial conditions are given at the initial high-energy cutoff $\omega_{c}^{0}$ :

$$
\begin{aligned}
h\left(\omega_{c}^{0}\right) & =0 \\
J_{\alpha_{1} \alpha_{2}, \omega_{1}, \omega_{2}}\left(\omega_{c}^{0}\right) & =J_{\alpha_{1} \alpha_{2}}, \\
K_{\alpha_{1} \alpha_{2}, \omega_{1}, \omega_{2}}\left(\omega_{c}^{0}\right) & =0
\end{aligned}
$$

Thus, since the impurity Hamiltonian stays degenerate, Eq. (5.20) does not depend on $H_{0}\left(\omega_{c}\right)$. Therefore, the running couplings are determined by Eq. (5.20) only. However, 
because of the degeneracy of $H_{0}$ we have to introduce a regularization parameter $\epsilon$ to perform the time integral. Defining the dimensionless couplings

$$
\begin{aligned}
& \bar{J}_{\alpha_{1} \alpha_{2}, \omega_{1}, \omega_{2}}:=\rho_{\alpha_{1}} J_{\alpha_{1} \alpha_{2}, \omega_{1}+\mu_{\alpha_{1}, \omega_{2}}+\mu_{\alpha_{2}}}, \\
& \bar{K}_{\alpha_{1} \alpha_{2}, \omega_{1}, \omega_{2}}:=\rho_{\alpha_{1}} K_{\alpha_{1} \alpha_{2}, \omega_{1}+\mu_{\alpha_{1}, \omega_{2}}+\mu_{\alpha_{2}}}
\end{aligned}
$$

we obtain the $R G$ equations

$$
\begin{gathered}
\frac{d \bar{J}_{\alpha_{1} \alpha_{2}, \omega_{1}, \omega_{2}}=-\int d \omega \sum_{\alpha}}{d \omega_{c}} \frac{d q\left(\omega, \omega_{c}\right)}{d \omega_{c}}\left\{\left(\frac{e^{\omega / D} \Theta(-\omega)}{\bar{\omega}-\omega+\bar{V}_{\alpha}-i \epsilon}-\frac{e^{-\omega / D} \Theta(\omega)}{\bar{\omega}-\omega+\bar{V}_{\alpha}+i \epsilon}\right)\right. \\
\quad \times \bar{J}_{\alpha_{1} \alpha, \omega_{1}, \omega} \bar{J}_{\alpha \alpha_{2}, \omega, \omega_{2}} \\
+\left(\frac{e^{\omega / D} \Theta(-\omega)}{\bar{\omega}-\omega+\bar{V}_{\alpha}-i \epsilon}+\frac{e^{-\omega / D} \Theta(\omega)}{\bar{\omega}-\omega+\bar{V}_{\alpha}+i \epsilon}\right) \\
\left.\quad \times\left(\bar{J}_{\alpha_{1} \alpha, \omega_{1}, \omega} \bar{K}_{\alpha \alpha_{2}, \omega, \omega_{2}}+\bar{K}_{\alpha_{1} \alpha, \omega_{1}, \omega} \bar{J}_{\alpha \alpha_{2}, \omega, \omega_{2}}\right)\right\} \\
\frac{d \bar{K}_{\alpha_{1} \alpha_{2}, \omega_{1}, \omega_{2}}}{d \omega_{c}}=-\int d \omega \sum_{\alpha} \frac{d q\left(\omega, \omega_{c}\right)}{d \omega_{c}}\left(\frac{e^{\omega / D} \Theta(-\omega)}{\bar{\omega}-\omega+\bar{V}_{\alpha}-i \epsilon}+\frac{e^{-\omega / D} \Theta(\omega)}{\bar{\omega}-\omega+\bar{V}_{\alpha}+i \epsilon}\right) \\
\times\left(3 / 4 \bar{J}_{\alpha_{1} \alpha, \omega_{1}, \omega} \bar{J}_{\alpha \alpha_{2}, \omega, \omega_{2}}+\bar{K}_{\alpha_{1} \alpha, \omega_{1}, \omega} \bar{K}_{\alpha \alpha_{2}, \omega, \omega_{2}}\right)
\end{gathered}
$$

Here, we introduced the parameters

$$
\begin{aligned}
\bar{\omega} & =1 / 2\left(\omega_{1}+\omega_{2}\right), \\
\bar{V}_{\alpha} & =1 / 2\left(\mu_{\alpha_{1}}-\mu_{\alpha}\right)+1 / 2\left(\mu_{\alpha_{2}}-\mu_{\alpha}\right) .
\end{aligned}
$$

One now has to make a choice for the cutoff-function $q\left(\omega, \omega_{c}\right)$. The standard choice would be a sharp cutoff, which reads

$$
q\left(\omega, \omega_{c}\right)=\Theta\left(\omega+\omega_{c}\right)-\Theta\left(\omega-\omega_{c}\right)
$$

so that

$$
\frac{d q\left(\omega, \omega_{c}\right)}{d \omega_{c}}=\delta\left(\omega+\omega_{c}\right)+\delta\left(\omega-\omega_{c}\right) .
$$

Substituting this into Eqs. (5.28) and (5.29), however generates divergencies for $\epsilon \rightarrow 0$. If one uses a smooth cutoff, one deals with a principal value integral instead of divergencies, but consequently, one then has to solve integro-differential equations. This has been an inherent problem of the RTRG in energy space. However, one may obtain a well-defined $\mathrm{RG}$ scheme represented only by differential equations, if one uses a more general form of $q\left(\omega, \omega_{c}\right)$ by allowing for dependences on the external vertices $\left(\alpha_{1}, \alpha_{2}, \omega_{1}, \omega_{2}\right)$ as well as a further dependence on the contraction index $\alpha$. We define

$$
\begin{aligned}
\tilde{q}\left(\omega, \omega_{c}\right) & =\Theta\left(\omega-\bar{\omega}-\bar{V}_{\alpha}+\omega_{c}\right)-\Theta\left(\omega-\bar{\omega}-\bar{V}_{\alpha}-\omega_{c}\right), \\
\Rightarrow \frac{d \tilde{q}\left(\omega, \omega_{c}\right)}{d \omega_{c}} & =\delta\left(\omega-\bar{\omega}-\bar{V}_{\alpha}+\omega_{c}\right)+\delta\left(\omega-\bar{\omega}-\bar{V}_{\alpha}-\omega_{c}\right) .
\end{aligned}
$$




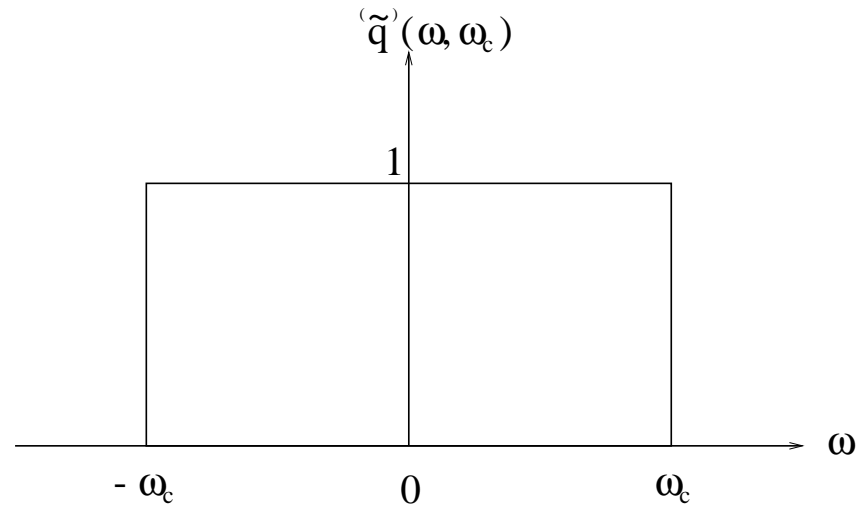

$$
\left(\bar{\omega}+\bar{V}_{\alpha}-\omega_{c}\right) \quad\left(\bar{\omega}+\bar{V}_{\alpha}\right) \quad\left(\bar{\omega}+\bar{V}_{\alpha}+\omega_{c}\right)
$$

Figure 5.2: The cutoff function in energy space. The standard choice $q\left(\omega, \omega_{c}\right)$ and our new choice $\tilde{q}\left(\omega, \omega_{c}\right)$ (in brackets). The new sharp cutoff is shifted by $\bar{\omega}+\bar{V}_{\alpha}$.

This choice is again a sharp cutoff. But whereas in case of Eq. (5.32) the cutoff is centered around 0 (with respect to the chemical potential of the contraction reservoir), the definition in Eq. (5.34) is centered around $\bar{\omega}+\bar{V}_{\alpha}$ (see Fig. 5.2). Thereby the divergencies for $\epsilon \rightarrow 0$ are avoided for $\omega_{c}>0$. With the definition

$$
\Delta\left(\omega_{c}\right)=\omega_{c}+\bar{\omega}+\bar{V}_{\alpha}
$$

we obtain

$$
\begin{aligned}
& \frac{d \bar{J}_{\alpha_{1} \alpha_{2}, \omega_{1}, \omega_{2}}}{d \ln \omega_{c}}=\sum_{\alpha}\left\{-\operatorname{sign}(\Delta) \bar{J}_{\alpha_{1} \alpha, \omega_{1}, \Delta} \bar{J}_{\alpha \alpha_{2}, \Delta, \omega_{2}}\right. \\
& \left.+\left(\bar{J}_{\alpha_{1} \alpha, \omega_{1}, \Delta} \bar{K}_{\alpha \alpha_{2}, \Delta, \omega_{2}}+\bar{K}_{\alpha_{1} \alpha, \omega_{1}, \Delta} \bar{J}_{\alpha \alpha_{2}, \Delta, \omega_{2}}\right)\right\} \\
& -\sum_{\alpha}\left\{\quad \Delta\left(\omega_{c}\right) \rightarrow \Delta\left(-\omega_{c}\right) \quad\right\} \\
& \frac{d \bar{K}_{\alpha_{1} \alpha_{2}, \omega_{1}, \omega_{2}}}{d \ln \omega_{c}}=\sum_{\alpha}\left\{\left(3 / 4 J_{\alpha_{1} \alpha, \omega_{1}, \Delta} \bar{J}_{\alpha \alpha_{2}, \Delta, \omega_{2}}+\bar{K}_{\alpha_{1} \alpha, \omega_{1}, \Delta} \bar{K}_{\alpha \alpha_{2}, \Delta, \omega_{2}}\right)\right\} \\
& -\sum_{\alpha}\left\{\quad \Delta\left(\omega_{c}\right) \rightarrow \Delta\left(-\omega_{c}\right)\right\},
\end{aligned}
$$

where we have used $\Theta(x)-\Theta(-x)=\operatorname{sign}(x)$ and $\Theta(x)+\Theta(-x)=1$. Furthermore, we have set $D \rightarrow \infty$, so that the initial high-energy cutoff $\omega_{c}^{0}$ plays the role of the bandwidth. The above equations fulfill the symmetry relations

$$
\begin{aligned}
\bar{J}_{\alpha_{1} \alpha_{2}, \omega_{1}, \omega_{2}} & =\bar{J}_{\bar{\alpha}_{1} \bar{\alpha}_{2},-\omega_{1},-\omega_{2}}, \\
\bar{K}_{\alpha_{1} \alpha_{2}, \omega_{1}, \omega_{2}} & =-\bar{K}_{\bar{\alpha}_{1} \bar{\alpha}_{2},-\omega_{1},-\omega_{2}}, \\
\bar{J}_{\alpha_{1} \alpha_{2}, \omega_{1}, \omega_{2}} & =\bar{J}_{\alpha_{2} \alpha_{1}, \omega_{2}, \omega_{1}}, \\
\bar{K}_{\alpha_{1} \alpha_{2}, \omega_{1}, \omega_{2}} & =\bar{K}_{\alpha_{2} \alpha_{1}, \omega_{2}, \omega_{1}} .
\end{aligned}
$$




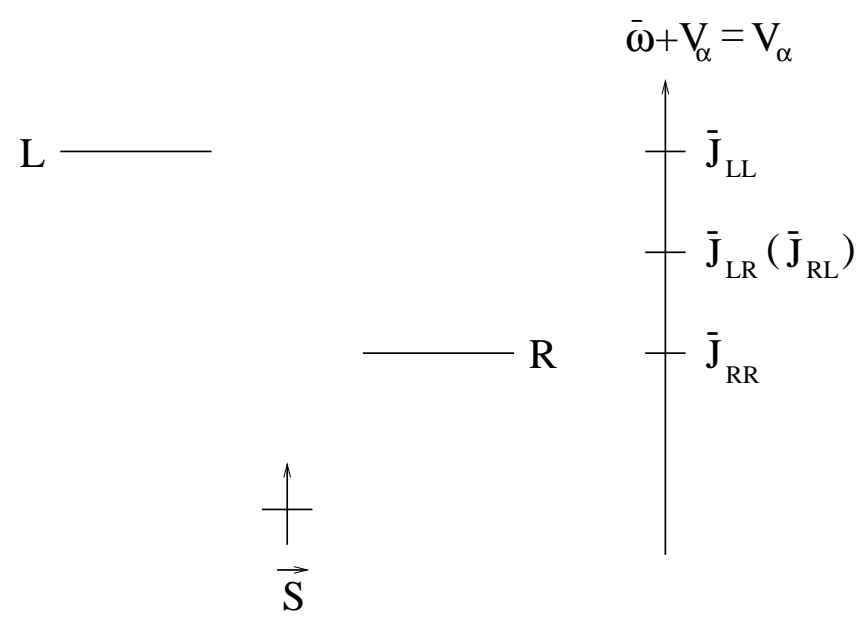

Figure 5.3: The center of the cutoff-function, $\bar{\omega}+\bar{V}_{\alpha}$, for the different coupling constants $\bar{J}_{L L}, \bar{J}_{R R}$ and $\bar{J}_{L R}\left(\bar{J}_{R L}\right)$ with $\bar{\omega}=\omega_{1}=\omega_{2}=0$. This applies in the same way for the couplings $\bar{K}$.

By $\bar{\alpha}$ we denote the opposite electron reservoir of the band $\alpha$. Let us consider the RG flow of the coupling constants in the low-energy limit, i.e. $\omega_{1}=\omega_{2}=0$. The energies, around which the cutoff-functions are centered, can be seen from Fig. 5.3. They are different for the reflection coefficients $\bar{J}_{L L}\left(\bar{K}_{L L}\right), \bar{J}_{R R}\left(\bar{K}_{R R}\right)$ and the transmission constants $\bar{J}_{L R}$ $\left(\bar{K}_{L R}\right), \bar{J}_{R L}\left(\bar{K}_{R L}\right)$.

\subsection{The running couplings}

\subsubsection{The role of the external voltage}

In Fig. 5.3 one recognizes that due to the occupancy in the bands at $T=0$, the renormalization-group flow should qualitatively change at $\omega_{c}=V\left(\omega_{c}=V / 2\right)$ regarding the reflection (transmission) couplings. This can be studied for the couplings $\bar{J}$ by neglecting the coefficients $\bar{K}$, which will be justified later in an exact solution. Then 
Eq. (5.37) yields for $\omega_{1}=\omega_{2}=0$

$$
\begin{aligned}
& \frac{d \bar{J}_{L L, 0,0}}{d \ln \omega_{c}}=\frac{d \bar{J}_{R R, 0,0}}{d \ln \omega_{c}} \\
& =-\left(\bar{J}_{L L, 0, \omega_{c}} \bar{J}_{L L, \omega_{c}, 0}+\bar{J}_{L L, 0,-\omega_{c}} \bar{J}_{L L,-\omega_{c}, 0}\right) \\
& -\left(\bar{J}_{L R, 0, V+\omega_{c}} \bar{J}_{R L, V+\omega_{c}, 0}+\bar{J}_{L R, 0, V-\omega_{c}} \bar{J}_{R L, V-\omega_{c}, 0}\right) \\
& \text { for } \omega_{c}>V \text {, } \\
& \frac{d \bar{J}_{L L, 0,0}}{d \ln \omega_{c}}=\frac{d \bar{J}_{R R, 0,0}}{d \ln \omega_{c}} \\
& =-\left(\bar{J}_{L L, 0, \omega_{c}} \bar{J}_{L L, \omega_{c}, 0}+\bar{J}_{L L, 0,-\omega_{c}} \bar{J}_{L L,-\omega_{c}, 0}\right) \\
& -\left(\bar{J}_{L R, 0, V+\omega_{c}} \bar{J}_{R L, V+\omega_{c}, 0}-\bar{J}_{L R, 0, V-\omega_{c}} \bar{J}_{R L, V-\omega_{c}, 0}\right) \\
& \text { for } \omega_{c}<V \text {, } \\
& \frac{d \bar{J}_{L R, 0,0}}{d \ln \omega_{c}}=\frac{d \bar{J}_{R L, 0,0}}{d \ln \omega_{c}} \\
& =-\left(\bar{J}_{L L, 0,-V / 2+\omega_{c}} \bar{J}_{L R,-V / 2+\omega_{c}, 0}+\bar{J}_{L L, 0,-V / 2-\omega_{c}} \bar{J}_{L R,-V / 2-\omega_{c}, 0}\right) \\
& -\left(\bar{J}_{L R, 0, V / 2+\omega_{c}} \bar{J}_{R R, V / 2+\omega_{c}, 0}+\bar{J}_{L R, 0, V / 2-\omega_{c}} \bar{J}_{R R, V / 2-\omega_{c}, 0}\right) \\
& \text { for } \omega_{c}>V / 2 \text {, } \\
& \frac{d \bar{J}_{L R, 0,0}}{d \ln \omega_{c}}=\frac{d \bar{J}_{R L, 0,0}}{d \ln \omega_{c}} \\
& =\left(\bar{J}_{L L, 0,-V / 2+\omega_{c}} \bar{J}_{L R,-V / 2+\omega_{c}, 0}-\bar{J}_{L L, 0,-V / 2-\omega_{c}} \bar{J}_{L R,-V / 2-\omega_{c}, 0}\right) \\
& -\left(\bar{J}_{L R, 0, V / 2+\omega_{c}} \bar{J}_{R R, V / 2+\omega_{c}, 0}-\bar{J}_{L R, 0, V / 2-\omega_{c}} \bar{J}_{R R, V / 2-\omega_{c}, 0}\right) \\
& \text { for } \omega_{c}<V / 2 \text {. }
\end{aligned}
$$

If one neglects the frequency-dependence in the above equations, one obtains the RG flow proposed by Coleman et al. (Eqs. (5.6) and (5.7)). Thus, $V$ acts as a cutoff for the running couplings giving rise to a two-channel behaviour, in which the couplings $\bar{J}_{L L}$ and $\bar{J}_{R R}$ are renormalized independently by intra-lead processes and the transmission terms are effectively suppressed. But as we have already mentioned, a finite external voltage also gives rise to rates which we did not account for here. These rates may act as a further cutoff which may destroy the two-channel physics [43].

\subsubsection{Exact results}

In this subsection we present the exact solution of the set of ordinary differential equations, Eqs. (5.37) and (5.38). We again use the numerical method described in Appendix E.1. The initial high-energy cutoff $\omega_{c}^{0}=1000$ sets the energy scale of the problem. For the initial value of $\bar{J}\left(=\bar{J}_{L L}=\bar{J}_{R R}=\bar{J}_{L R}=\bar{J}_{R L}\right)$ we choose 0.01 , so that $T_{K} \approx 10^{-9}$. For the solution we choose a logarithmic discretization of the frequencies $\omega_{1}$ respectively $\omega_{2}$. It turns out, that it is sufficient to take into account 50 frequencies. The running couplings for $\omega_{1}=\omega_{2}=0$ both for the biased and the unbiased case are 


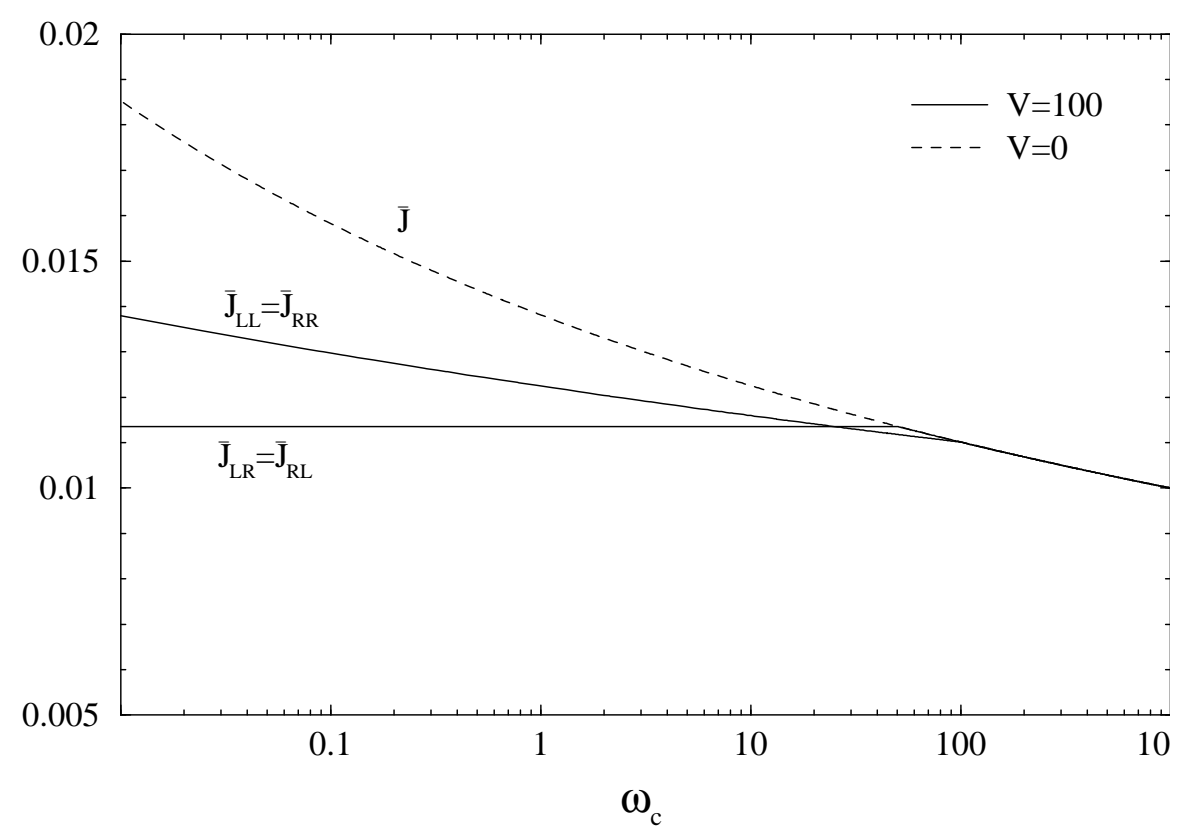

Figure 5.4: RG flow of the coupling constants $\bar{J}_{\alpha_{1} \alpha_{2}}$ for $\omega_{1}=\omega_{2}=0$.

Solid line: $V=100$. Dashed line: $V=0$.

shown in Figs. 5.4 and 5.5. For $V=0$, Eqs. (5.37) and (5.38) conserve the symmetry $\bar{J}=\bar{J}_{L L}=\bar{J}_{R R}=\bar{J}_{L R}=\bar{J}_{R L}$, which also applies to the couplings $\bar{K}$. Hence, with the symmetry relations in Eqs. (5.39) one recognizes that $\bar{K}=0$ holds (not shown in Fig. 5.5). For $V=100$ the couplings stay the same up to $\omega_{c} \approx V$. For $\omega_{c}<V$ one recognizes the different flow for the reflection coefficients and the transmission coefficients. $\bar{K}_{L L}$ and $\bar{K}_{R R}$ are generated, but they are negligible compared to the couplings $\bar{J}$. To compare our solution with the proposal of Coleman et al. in detail we plot $1 / \bar{J}_{\alpha_{1} \alpha_{2}}$ (see Fig. 5.6). For the unbiased case we obtain the correct single-lead behaviour given by Eq. (5.5). For $V=100$ one recognizes that the renormalization strength of the inverse reflection coefficients is decreased by a factor of 2 when $\omega_{c}$ crosses $V$, whereas the transmission coefficients stay constant for $\omega_{c}<V / 2$. This corresponds to the same two-channel behaviour that we already obtained from our approximation in the previous subsection. Thus the RG flow confirms the proposal of Coleman et al., Eqs. (5.6) and (5.7). The slope of the couplings $J$ is discontinuous, since we study the case of vanishing temperature. However, the stationary current in case of finite $V$ corresponds to a rate $\Gamma$ which we neglected. Thus the presented RG flow only holds for $\omega_{c}>\Gamma$.

The frequency-dependence of $\bar{J}$ and $\bar{K}$ for $V=0$ and $\omega_{c}=0.01$ is shown in Figs. 5.7 and 5.8. For $\bar{J}$ we obtain a peak structure, where the peaks are located at $\bar{\omega}=0$. This is due to our definition of the cutoff function $\tilde{q}\left(\omega, \omega_{c}\right)$, where $\bar{\omega}$ acts in the same way as $\bar{V}_{\alpha}$. Hence, both a finite $V$ and a finite $\bar{\omega}$ act as a cutoff for the RG flow. From Fig. 5.8 we see that $|\bar{K}|<10^{-4}$ holds for all frequencies, which justifies the neglecting of the couplings $\bar{K}$ in Subsection 5.3.1. In Figs. 5.9 and 5.10 we show the frequency-dependence of $\bar{J}_{L L}$ 


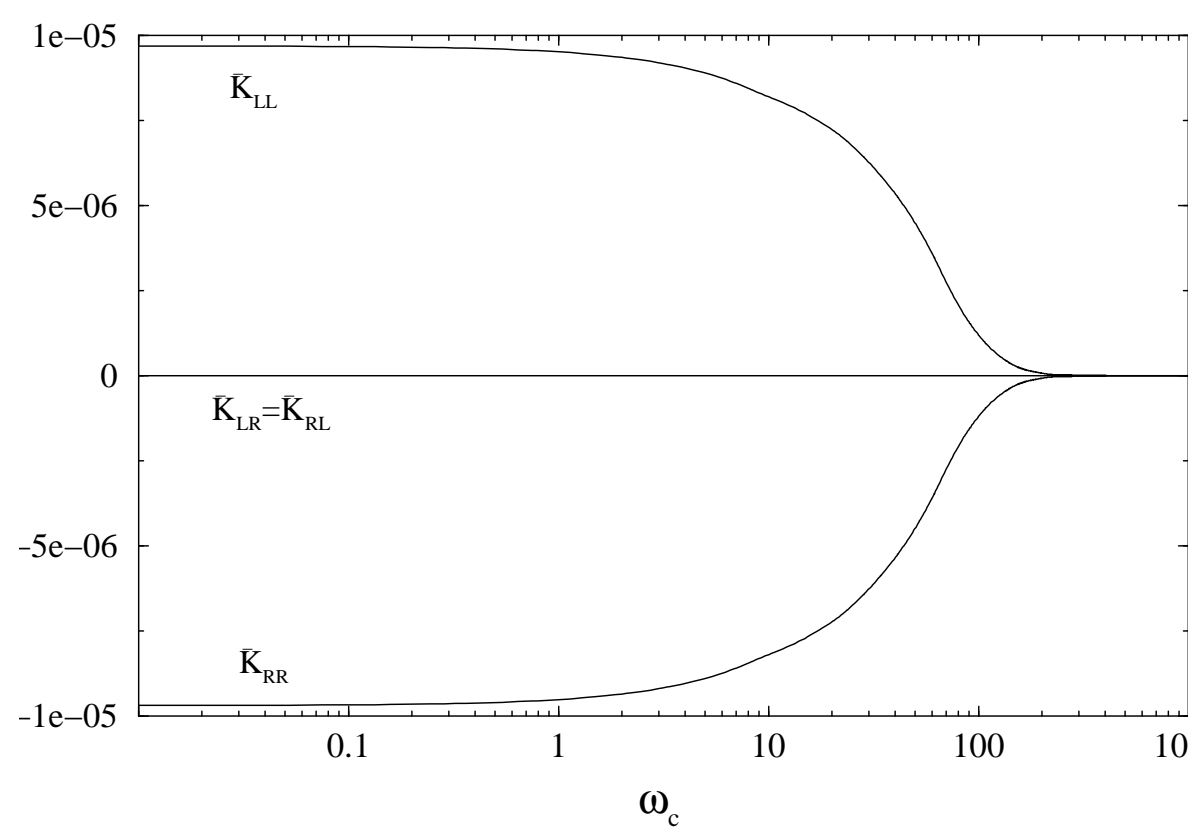

Figure 5.5: RG flow of the coupling constants $\bar{K}_{\alpha_{1} \alpha_{2}}$ for $\omega_{1}=\omega_{2}=0$ and $V=100$.

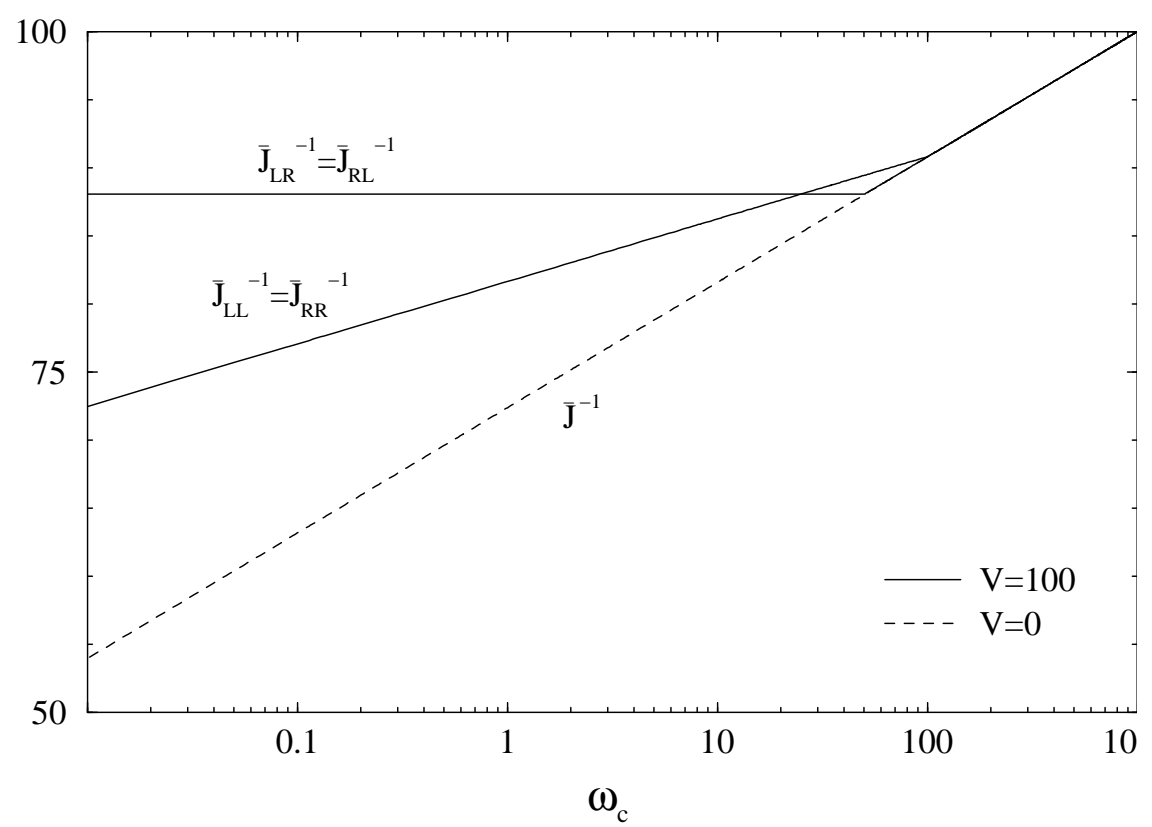

Figure 5.6: RG flow of the coupling constants $\bar{J}_{\alpha_{1} \alpha_{2}}^{-1}$ for $\omega_{1}=\omega_{2}=0$.

Solid line: $V=100$. Dashed line: $V=0$. 


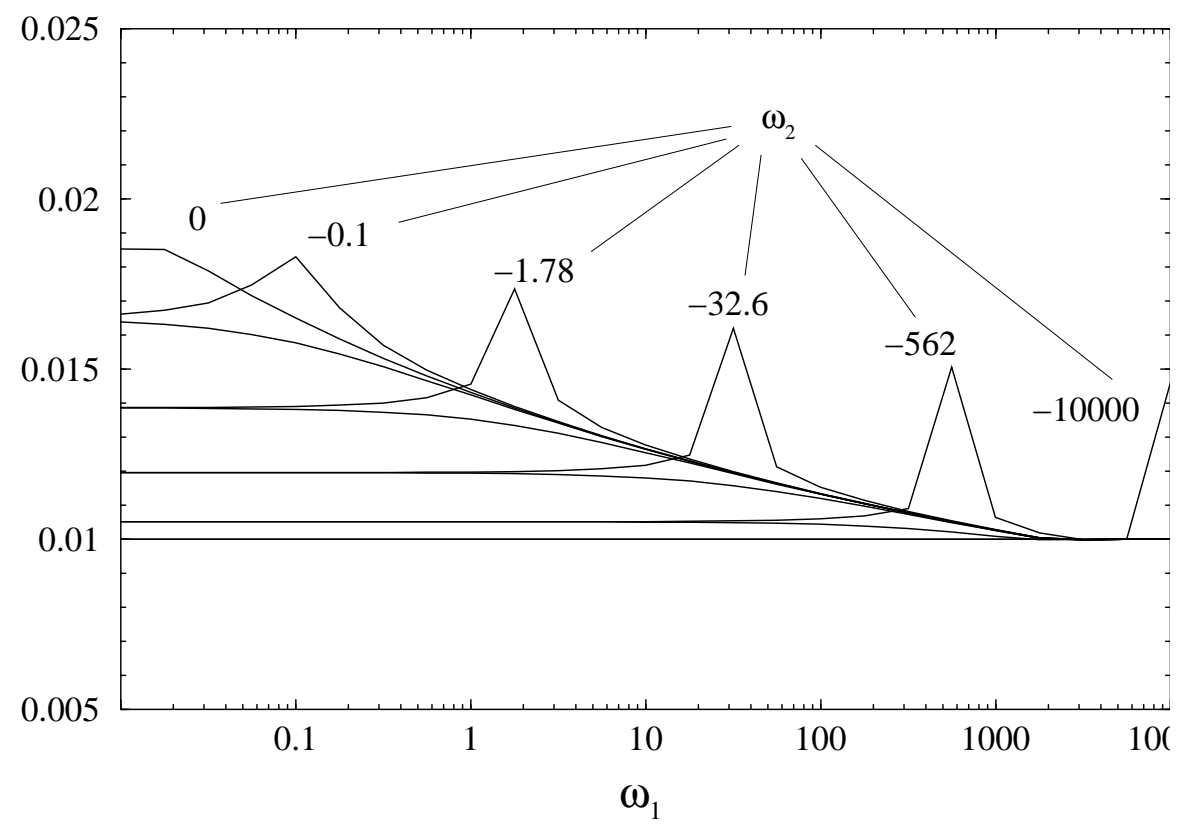

Figure 5.7: Coupling constant $\bar{J}$ as a function of frequency $\omega_{1}$ for different $\omega_{2}$ with $V=0$ and $\omega_{c}=0.01$.

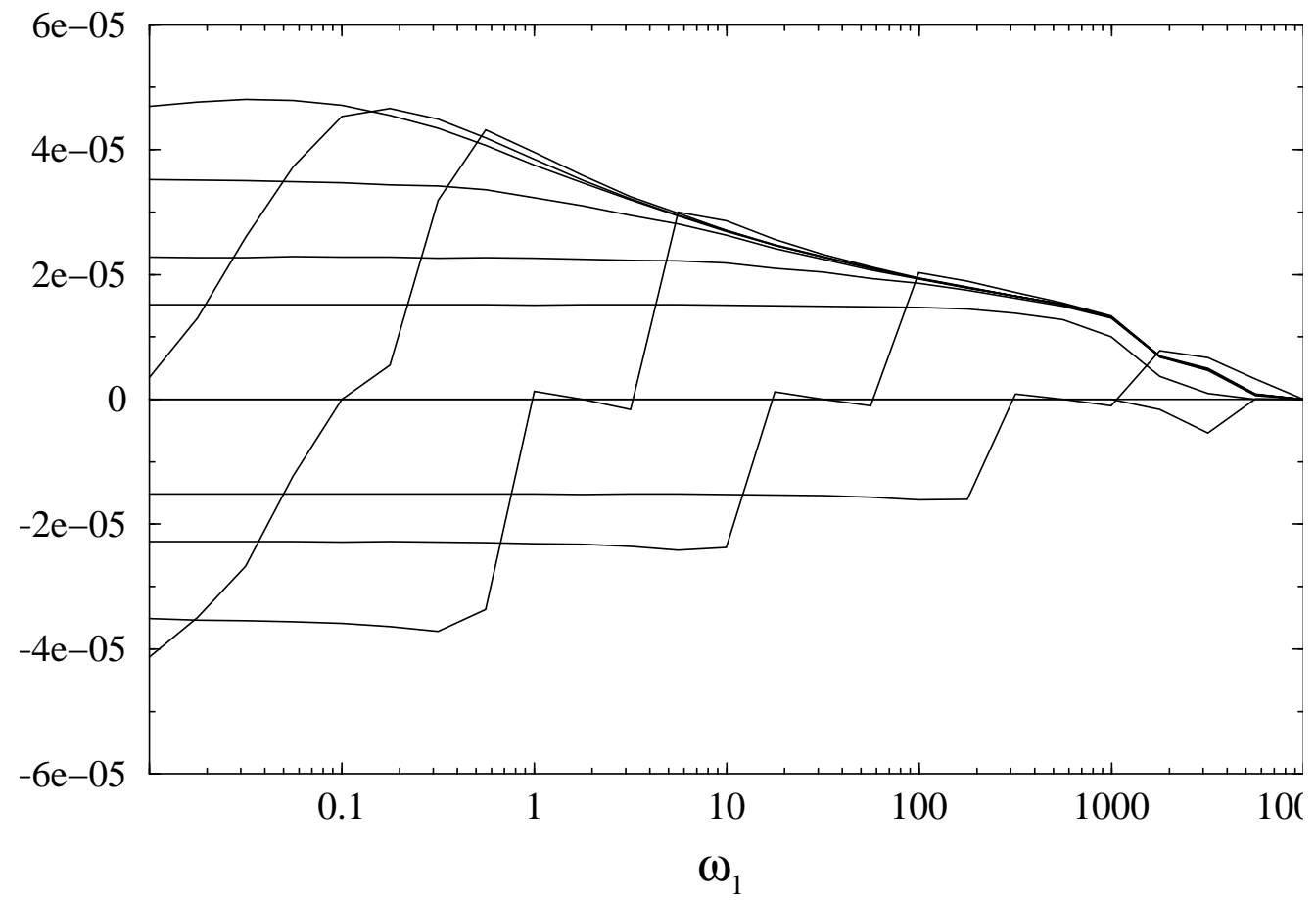

Figure 5.8: Coupling constant $\bar{K}$ as a function of frequency $\omega_{1}$ for different $\omega_{2}$ with $V=0$ and $\omega_{c}=0.01$. 


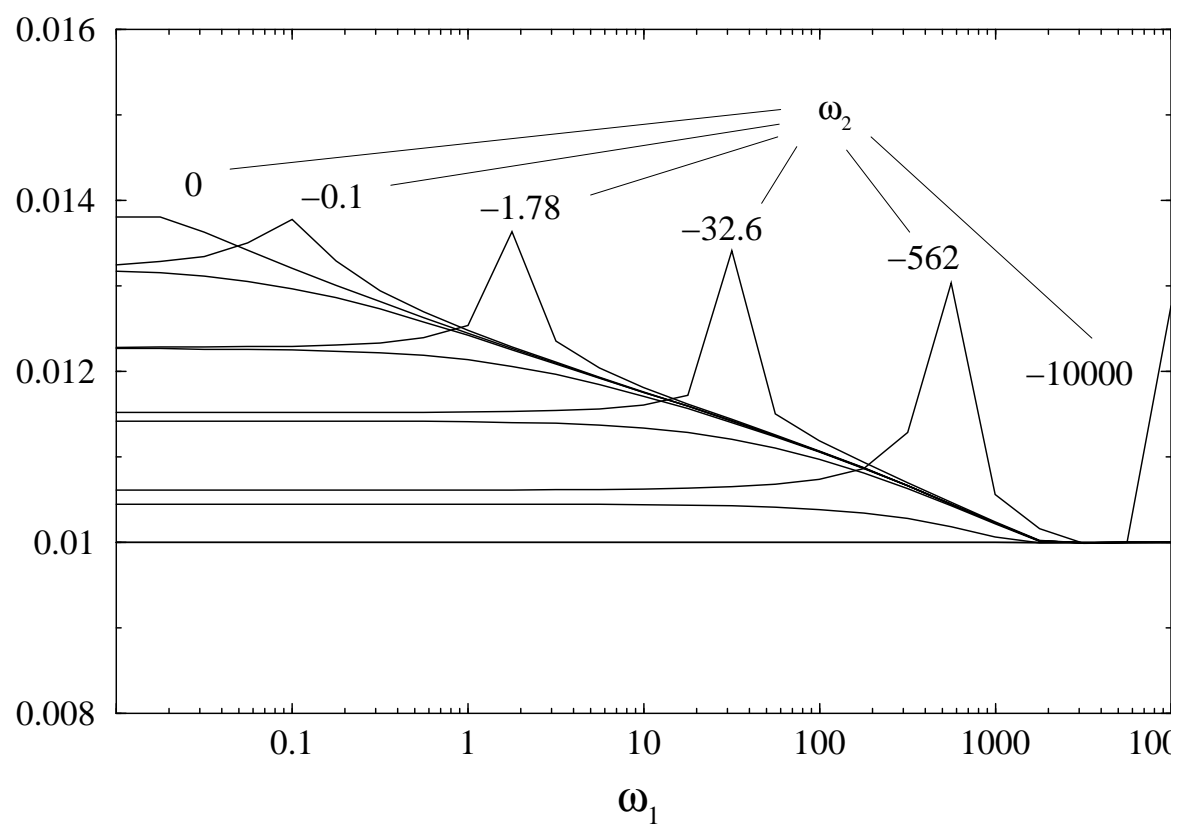

Figure 5.9: Coupling constant $\bar{J}_{L L}$ as a function of frequency $\omega_{1}$ for different $\omega_{2}$ with $V=100$ and $\omega_{c}=0.01$.

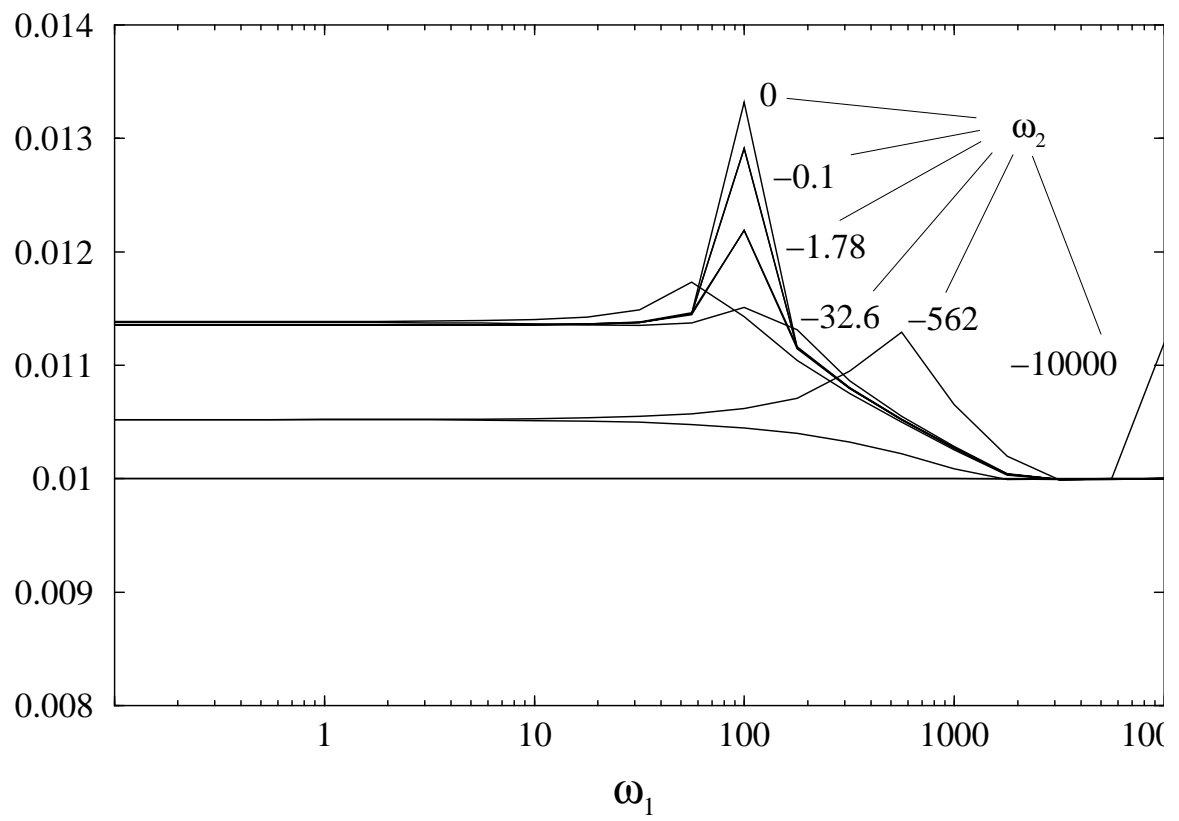

Figure 5.10: Coupling constant $\bar{J}_{L R}$ as a function of frequency $\omega_{1}$ for different $\omega_{2}$ with $V=100$ and $\omega_{c}=0.01$. 
and $\bar{J}_{L R}$ for $V=100$ and $\omega_{c}=0.01 . \bar{J}_{L L}$ is smaller than for the unbiased case, but it shows the same peak structure. In contrast, for $\bar{J}_{L R}$ the peaks are concentrated around $\omega_{1}=V$.

\subsection{Discussion}

In summary, we have applied the RTRG to the two-lead Kondo model and studied the renormalization-group flow of the coupling constants quantitatively. Since the Kondo problem involves double vertex objects, we have used a formulation of the RTRG in energy space. Thereby the retardation effects are accounted for without an additional timedependence, i.e. the generation of integro-differential equations is avoided. However, this formulation naturally leads to divergencies in the RG equations. To set up a well-defined RG scheme, we have developed a technique by which the singularities arising in energy space are avoided. Studying an effective Hamiltonian for $T=0$ we obtained the correct renormalization-group flow for the single-lead Kondo model, i.e. for vanishing bias. For the case of finite bias we quantitatively found a two-channel behaviour of the running couplings, which had been proposed earlier in a qualitative analysis [37].

However, since we studied only one propagator, we did not take effects into account, which can only be explained by considering the Keldysh contour. Thus, we disregarded rates which arise for a finite voltage $V$. A rate $\Gamma$ may act as a further cutoff, so that the obtained RG flow is cut off at $\omega_{c} \approx \Gamma$. Depending on the magnitude of $\Gamma$ this may destroy the two-channel physics [38]. Thus, a complete analysis of the two-lead Kondo model would require a study of the Keldysh contour. We have already presented the formulation of the RTRG for the Keldysh contour (see Section 3.2). This is also applicable in energy space, and again any divergencies can be avoided by applying our generalization of the cutoff-function (the RG equations are presented in Appendix D). However, one then deals with $(4 \times 4)$-matrices. Despite certain symmetries this results in a too large problem size. The numerical solution for only 10 frequencies on a PIII (500MHz)-computer then requires roughly 100 days. 


\section{Chapter 6}

\section{Conclusion}

In this thesis we used renormalization-group (RG) theory to study different quantum dissipative systems, which are formed by a local system interacting with a dissipative environment. By applying the recently developed real-time renormalization-group (RTRG) method [12] we have not been restricted to equilibrium considerations, but we also examined both stationary states in out-of-equilibrium situations and the time-dependent reduced density matrix, where the latter describes the time evolution of the local system out of an arbitrary nonequilibrium state.

As a starting point we applied the RTRG to the polaron problem, a standard problem of many-particle theory, where we also drew comparisons to various methods including the flow equation formalism of Wegner [3]. We considered the one-dimensional case and calculated the ground-state energy and the effective mass for vanishing temperature [24]. Thus, we were able to use the Gell-Mann-Low theorem, and a full nonequilibrium consideration on the Keldysh contour was not necessary. It turned out that the standard approximation of the flow equations for that problem [49] led to worse results than simple perturbation theory. On the other hand, the RTRG method was able to describe the polaron beyond perturbation theory. However, there problems arose from the form of the bath correlations, which did not decay. As a consequence we had to apply a physically motivated approximation, and we achieved only a low accuracy for the ground-state energy and the effective mass. Moreover, in case of the polaron the local system consisted of a continuous electron spectrum, so that a study of the reduced density matrix was numerically impossible.

This motivated us to study a more fundamental, yet nontrivial quantum dissipative system, the spin-boson model, where a two-state system is coupled to a bosonic heat bath. For this model the dynamics of the local system in the presence of quantum dissipation could be determined. Since in this case the bath contractions were decaying we obtained a convergent RG flow. Before we applied the RTRG to the spin-boson model, we presented a detailed explanation of the formalism for arbitrary nonequilibrium situations, i.e. involving the Keldysh contour, and we also extended the RG scheme to the calculation of equilibrium correlation functions. Within the RTRG formalism the explicit cutoff-dependence of the bath contractions $\left(\gamma^{p_{1}, p_{2}}\left(t, t_{c}\right)\right)$ is not specified. For the spin- 
boson model we discussed different choices of $\gamma^{p_{1}, p_{2}}\left(t, t_{c}\right)$. We showed that an adequate definition was necessary in this case, because the standard choice of a sharp cutoff led to large errors, which could be avoided by applying a modified definition of $\gamma^{p_{1}, p_{2}}\left(t, t_{c}\right)$.

An important advantage of renormalization-group theory is the possibility to study a physical problem beyond perturbation theory. In case of the spin-boson model this means, that the correct renormalized tunnel matrix element is accounted for. For the RTRG we demonstrated that in an analytical calculation.

We solved the RG equations numerically to determine stationary quantities, such as the static susceptibility, and the time-dependent reduced density matrix $p(t)$, starting from an arbitrary nonequilibrium state. For $p(t)$ we also analyzed the oscillation frequency as well as the asymptotic behaviour. Furthermore, we calculated equilibrium correlation functions both in the frequency and the time domain $[25,26]$. The reliability of our results was shown by the consistency with chromostochastic quantum dynamics (CSQD), and the good agreement with the exact Shiba-relation. Furthermore, we checked the correct scaling behaviour of the spin-boson model. We obtained accurate results for arbitrary parameters with the only restriction of not too large couplings to the environment. The latter is due to the approximation, where we neglected double and higher-order vertex objects.

In contrast to the noninteracting blip approximation (NIBA), our approach allowed the calculation of the dynamics of the complete reduced density matrix. Moreover, the regime of validity of the NIBA is characterized by further restrictions regarding the parameters of the model. We also compared our results to those obtained for the anisotropic Kondo model. Since we could choose arbitrary parameters these comparisons could be made in a parameter regime, where the mapping of the spin-boson model on the anisotropic Kondo model cannot be proven rigorously.

The results for the spin-boson model demonstrated that the RTRG is a powerful method, which is easily generalized to other quantum dissipative systems, where the neglecting of double vertex objects is justified.

We also studied a double quantum dot system, which is coupled to two electron leads and to a phonon environment. There a finite external voltage gives rise to a stationary tunnel current, which was measured in a recent experiment [30]. Applying the RTRG to that problem we could examine this out-of-equilibrium situation beyond perturbation theory. Using a formulation of the RTRG for expectation values of operators, which are linear in the bath operators, we set up the RG equations for the stationary tunnel current. For the experimentally realized coupling parameters, which were only moderately large, we obtained quantitatively reliable results. In contrast to previous theoretical studies [34] we could well describe the experimental data. We found that the finite extension of the electron densities within the quantum dots cannot be neglected, and we presented its dependence on the energy difference between the dots [35].

At the end of this thesis we discussed the two-lead Kondo model, where an impurity is coupled to two electron bands, between which a finite voltage may be applied. This is a model, where double vertices are important. Thus, the standard approximation, where one neglects double vertex objects, is not applicable. However, taking these objects into 
account leads to RG equations which are much more complicated: instead of ordinary differential equations one deals with integro-differential equations. This principal difficulty has been overcome by introducing a formulation of the RTRG in energy space. However, then the standard form of the RG equations involves divergencies. Using a generalized definition for the cutoff-dependence in the RG, we found a systematic way to avoid these divergencies. Thereby, we obtained well-defined RG equations for an effective Hamiltonian. The solution showed a two-channel behaviour of the running couplings, which was previously proposed but was not derived quantitatively [37]. However, the influence of rates, which arise from the stationary current in case of a finite voltage, was not taken into account, since we did not study the problem on the Keldysh contour. Such an analysis requires too much numerical effort, as we deal with an additional frequency-dependence of the vertex objects, when the RTRG is formulated in energy space.

Thus, it was shown, that also double (and higher-order) vertex objects can be treated within the RTRG, when one accounts for an additional frequency-dependence. Then the RG scheme is given by a set of ordinary differential equations. In principle, such equations can easily be solved numerically. However, a solution may become too timeconsuming, if the problem involves a too large problem size, which follows from the number of degrees of freedom in the local system and the number of vertices. In this context, note that, if one accounts for double vertex objects, an additional frequency-dependence is generated, which increases the numerical effort substantially.

In summary, the RTRG provides a new possibility to study quantum dissipative systems beyond perturbation theory; unlike other RG procedures, within the RTRG also arbitrary nonequilibrium quantities can be calculated. The physics of rates giving rise to non-Hamiltonian dynamics is included in this approach, and all time scales are accounted for, since the propagators have not been expanded. Applying this method we were able to present the first RG calculation of a time-dependent reduced density matrix. This was achieved for the spin-boson model, for which we determined various quantities, whose accuracy was high compared to other methods (see Subsection 3.4.4). Furthermore, we presented a quantitatively reliable solution for the stationary tunnel current through a double dot system in a phonon environment, and we systematically calculated the running couplings for the two-lead Kondo model. Both results could not be obtained before. In case of the double dot system it became also clear, that within the RTRG method different reservoirs can easily be treated since they do not lead to qualitatively more complicated RG equations. Therefore, in an outlook this method can also be used to study the phenomenon of thermal transport, wich arises for two baths at different temperatures. 



\section{Appendix A}

\section{The noninteracting blip approximation}

The noninteracting blip approximation (NIBA) [27] gives an approximate result for the time-dependent reduced density matrix $p(t)=\operatorname{Tr}_{B} \rho(t)$ for the spin-boson model given by the Hamiltonian in Eqs. (3.2), (3.5) and (3.6). In Chapter 3 we compared our result for $p(t)$ to that of the NIBA. The NIBA is most often used for the calculation of the diagonal elements of $p(t)$ for the unbiased case. However, we considered all elements of $p(t)$ for arbitrary $\epsilon$. Our analysis in Chapter 3 showed the restrictions of the NIBA regarding its validity for that general case. These findings were also important for our study of the coupled quantum dots in a phonon bath in Chapter 4.

Therefore, we here present the general formal results of the NIBA for the complete reduced density matrix for arbitrary parameters. The derivation is described in some detail in Ref. [1]. In the following we outline the main steps. As in Subsection 3.2.1 we assume a product initial state at $t=0$ :

$$
\rho(0)=p(0) \rho_{B}^{e q} .
$$

Following the method of Feynman and Vernon [62] we write $p(t)$ as a path-integral and account for the bath degrees of freedom by the influence functional $F$.

$$
p(t)=J_{F V}(t) p(0),
$$

where $J_{F V}$ is a superoperator in Liouville space. Its matrix elements are given by

$$
\left(J_{F V}\right)_{\sigma_{f} \sigma_{f}^{\prime}, \sigma_{i} \sigma_{i}^{\prime}}(t)=\int \mathcal{D} \sigma \int \mathcal{D} \sigma^{\prime} \exp \left(\frac{i}{\hbar}\left(S_{0}[\sigma]-S_{0}\left[\sigma^{\prime}\right]\right)\right) F\left[\sigma, \sigma^{\prime}\right] .
$$

The paths $\sigma$ jump between the states $|u\rangle(\sigma=1)$ and $|d\rangle(\sigma=-1)$, and the integration boundaries are given by

$$
\begin{aligned}
& \sigma(0)=\sigma_{i}, \quad \sigma(t)=\sigma_{f}, \\
& \sigma^{\prime}(0)=\sigma_{i}^{\prime}, \quad \sigma^{\prime}(t)=\sigma_{f}^{\prime} .
\end{aligned}
$$

$S_{0}$ is the action of the undamped two-state system. Let $m$ be the number of jumps between the two states on the path $\sigma$, and $m^{\prime}$ the corresponding number on the path $\sigma^{\prime}$. Then the 
path integrals for the free case can be written as $m+m^{\prime}$ time-integrals:

$$
\begin{gathered}
\int \mathcal{D} \sigma \int \mathcal{D} \sigma^{\prime} \exp \left(\frac{i}{\hbar}\left(S_{0}[\sigma]-S_{0}\left[\sigma^{\prime}\right]\right)\right) \\
=\sum_{m, m^{\prime}=0}^{\infty} \int_{0}^{t} d t_{1} \int_{t_{1}}^{t} d t_{2} \cdots \int_{t_{m+m^{\prime}-1}}^{t} d t_{m+m^{\prime}}\left(\frac{i}{\hbar} \frac{\Delta}{2}\right)^{m+m^{\prime}}(-1)^{m^{\prime}} \\
\times \exp \left(-\int_{0}^{t} d t^{\prime} \frac{i}{\hbar} \frac{\epsilon}{2}\left(\sigma\left(t^{\prime}\right)-\sigma^{\prime}\left(t^{\prime}\right)\right)\right) .
\end{gathered}
$$

The influence functional $F$ is obtained by integrating out the bath degrees of freedom:

$$
\begin{array}{r}
F\left[\sigma, \sigma^{\prime}\right]=\exp \left(\frac { 1 } { 4 } \int _ { 0 } ^ { t } d t ^ { \prime } \int _ { 0 } ^ { t ^ { \prime } } d t ^ { \prime \prime } \left(\left(\dot{\sigma}\left(t^{\prime}\right)-\dot{\sigma}^{\prime}\left(t^{\prime}\right)\right) \operatorname{Re}\left[Q\left(t^{\prime}-t^{\prime \prime}\right)\right]\left(\dot{\sigma}\left(t^{\prime \prime}\right)-\dot{\sigma}^{\prime}\left(t^{\prime \prime}\right)\right)\right.\right. \\
\left.\left.+i\left(\dot{\sigma}\left(t^{\prime}\right)-\dot{\sigma}^{\prime}\left(t^{\prime}\right)\right) \operatorname{Im}\left[Q\left(t^{\prime}-t^{\prime \prime}\right)\right]\left(\dot{\sigma}\left(t^{\prime \prime}\right)+\dot{\sigma}^{\prime}\left(t^{\prime \prime}\right)\right)\right)\right)
\end{array}
$$

with

$$
Q(t)=\int_{0}^{\infty} d \omega \frac{J(\omega)}{\pi \omega^{2}}\left(\operatorname{coth}\left(\frac{\hbar \omega}{2 k_{B} T}\right)(1-\cos (\omega t))+i \sin (\omega t)\right) .
$$

The spectral density $J(\omega)$ is given by Eq. (3.7) respectively Eq. (3.8). Comparing the expression for $Q(t)$ with the bath correlation function $\gamma(t)$ introduced in Eq. (3.15) one finds $\ddot{Q}(t)=\gamma(t)$. Correspondingly, in the limit of large high-energy cutoff $(D \rightarrow \infty)$ one obtains for the Ohmic bath

$$
Q(t)=2 \alpha \ln \left(\frac{D}{\pi k_{B} T} \sinh \left(\pi k_{B} T|t| / \hbar\right)\right)+i \pi \alpha \operatorname{sign}(t) .
$$

The spin paths $\sigma$ and $\sigma^{\prime}$ enter Eqs. (A.5) and (A.6) as combinations representing a diagonal state $\left(\sigma+\sigma^{\prime}\right)$ respectively an off-diagonal state $\left(\sigma-\sigma^{\prime}\right)$. When we start out from a diagonal state at $t_{0}=0$, we may write for a path for $\sigma$ and $\sigma^{\prime}$ with $2 n$ transitions at the time points $t_{j}(j=1, \ldots, 2 n)$

$$
\begin{aligned}
\frac{1}{2}\left(\sigma(t)+\sigma^{\prime}(t)\right) & =\sum_{j=0}^{n} \eta_{j}\left(\Theta\left(t-t_{2 j}\right)-\Theta\left(t-t_{2 j+1}\right)\right), \\
\frac{1}{2}\left(\sigma(t)-\sigma^{\prime}(t)\right) & =\sum_{j=1}^{n} \xi_{j}\left(\Theta\left(t-t_{2 j-1}\right)-\Theta\left(t-t_{2 j}\right)\right) .
\end{aligned}
$$

Here, the coefficients $\eta_{j}= \pm 1$ and $\xi_{j}= \pm 1$ depend on the diagonal respectively offdiagonal states. The time intervals $\left[t_{2 j}, t_{2 j+1}\right]$ are named sojourns, whereas the periods $\left[t_{2 j-1}, t_{2 j}\right]$ are called blips [27]. With the matrices

$$
\begin{array}{rr}
\Lambda_{j k}=\operatorname{Re}\left[Q\left(t_{2 j}-t_{2 k-1}\right)+Q\left(t_{2 j-1}-t_{2 k}\right)\right. \\
\left.-Q\left(t_{2 j}-t_{2 k}\right)-Q\left(t_{2 j-1}-t_{2 k-1}\right)\right] \\
X_{j k}=\operatorname{Im}\left[Q\left(t_{2 j}-t_{2 k+1}\right)+Q\left(t_{2 j-1}-t_{2 k}\right)\right. \\
\left.-Q\left(t_{2 j}-t_{2 k}\right)-Q\left(t_{2 j-1}-t_{2 k+1}\right)\right]
\end{array}
$$


the influence functional for the path in Eq. (A.9) is given by

$$
\begin{aligned}
F_{n}= & \exp \left(-\sum_{j=1}^{n} \operatorname{Re}\left[Q\left(t_{2 j}-t_{2 j-1}\right)\right]\right) \exp \left(-\sum_{j=2}^{n} \sum_{k=1}^{j-1} \xi_{j} \xi_{k} \Lambda_{j k}\right) \\
& \times \exp \left(i \sum_{k=0}^{n-1} \sum_{j=k+1}^{n} \xi_{j} \eta_{k} X_{j k}\right) .
\end{aligned}
$$

With Eq. (A.8) for $D \rightarrow \infty$ the last exponential in the above equation reads

$$
\exp \left(i \sum_{k=0}^{n-1} \sum_{j=k+1}^{n} \xi_{j} \eta_{k} X_{j k}\right)=\exp \left(i \sum_{k=1}^{n} \xi_{k} \eta_{k-1} \operatorname{Im}\left[Q\left(t_{2 k}-t_{2 k-1}\right)\right]\right) .
$$

The NIBA is based on the assumption that the average sojourn time is very large compared to the average blip time. Thus, in the NIBA one sets the interblip interactions $\Lambda_{j k}$ to zero. Then the influence functional reduces to the factorized form

$$
F_{n}^{\mathrm{NIBA}}=\prod_{j=1}^{n} \exp \left(-\operatorname{Re}\left[Q\left(t_{2 j}-t_{2 j-1}\right)\right]+i \xi_{j} \eta_{j-1} \operatorname{Im}\left[Q\left(t_{2 j}-t_{2 j-1}\right)\right]\right) .
$$

Within this approximation one may derive an expression for $p(t)$. According to Eq. (3.151) it is sufficient to consider the expectation values $\left\langle\sigma_{z}(t)\right\rangle$ and $\left\langle\sigma_{x}(t)\right\rangle$. Their Laplace transforms $\left\langle\sigma_{j}(z)\right\rangle=\int_{0}^{\infty} d t e^{i z t}\left\langle\sigma_{j}(t)\right\rangle \mathrm{read}$

$$
\begin{aligned}
\left\langle\sigma_{z}(z)\right\rangle & =\frac{1-\frac{i}{\hbar z} \Sigma_{z}^{(a)}(z)}{\Sigma_{z}^{(s)}(z)-i \hbar z-i \hbar z \Delta^{2} /\left(\epsilon^{2}-(\hbar z)^{2}\right)} \\
\left\langle\sigma_{x}(z)\right\rangle & =\frac{i}{\hbar z} \Sigma_{x}^{(s)}(z)-\left\langle\sigma_{z}(z)\right\rangle\left(\Sigma_{x}^{(a)}(z)+\epsilon \Delta /\left(\epsilon^{2}-(\hbar z)^{2}\right)\right) .
\end{aligned}
$$

Here, initially the system is prepared in the state $|u\rangle$ and we introduced the self-energies

$$
\begin{aligned}
& \Sigma_{z}^{(s)}(z)=\Delta^{2} \int_{0}^{\infty} d \tau e^{i z \tau} \cos (\epsilon \tau / \hbar)\left(e^{-\operatorname{Re}[Q(\tau)]} \cos (\operatorname{Im}[Q(\tau)])-1\right), \\
& \Sigma_{z}^{(a)}(z)=\Delta^{2} \int_{0}^{\infty} d \tau e^{i z \tau} \sin (\epsilon \tau / \hbar) e^{-\operatorname{Re}[Q(\tau)]} \sin (\operatorname{Im}[Q(\tau)]) \\
& \Sigma_{x}^{(s)}(z)=\Delta \int_{0}^{\infty} d \tau e^{i z \tau} \cos (\epsilon \tau / \hbar) e^{-\operatorname{Re}[Q(\tau)]} \sin (\operatorname{Im}[Q(\tau)]) \\
& \Sigma_{x}^{(a)}(z)=\Delta \int_{0}^{\infty} d \tau e^{i z \tau} \sin (\epsilon \tau / \hbar)\left(e^{-\operatorname{Re}[Q(\tau)]} \cos (\operatorname{Im}[Q(\tau)])-1\right)
\end{aligned}
$$

where $s(a)$ denotes whether the self-energies are (anti)symmetric under inversion of the bias $\epsilon$.

For the Ohmic bath the NIBA will provide accurate results for $\left\langle\sigma_{z}(t)\right\rangle$ in case of vanishing bias $\epsilon$ and weak coupling ( $\alpha \ll 1$ ). For, the contributions of the interblip correlations $\Lambda_{j k}$ to the self-energy $\Sigma_{z}^{(s)}$ cancel in first order in $\alpha$. Another regime of the Ohmic 
bath, where the NIBA is justified, is given by large damping and/or high temperature. This is due to the suppression of long blips because of the increase of $\operatorname{Re}[Q]$ with $t$ at long times. A more detailed study [1] yields that the temperature range, where the NIBA is valid, is given by

$$
\sqrt{\Delta_{r}^{2}+\epsilon^{2}} \lesssim k_{B} T
$$

Here, $\Delta_{r}=\Delta(\Delta / D)^{\alpha /(1-\alpha)}$ denotes the renormalized tunnel matrix element (see Eq. 3.11).

For $\epsilon=0$ and vanishing temperature one finds in the scaling limit (see Subsection 3.1)

$$
\left\langle\sigma_{z}(t)\right\rangle=E_{2-2 \alpha}\left(-\left(\Delta_{\mathrm{eff}} t / \hbar\right)^{2-2 \alpha}\right),
$$

where $E_{\nu}(z)$ is the Mittag-Leffler function [90] and

$$
\Delta_{\text {eff }}=(\Gamma(1-2 \alpha) \cos (\pi \alpha))^{1 / 2(1-\alpha)} \Delta_{r}
$$

For $\alpha<1 / 2$ this solution for $\left\langle\sigma_{z}(t)\right\rangle$ has an incoherent part, which decays as $1 /\left(\Delta_{\text {eff }} t / \hbar\right)^{2-2 \alpha}$, and a coherent part, which is proportional to $\cos (\Omega t) e^{-t / \tau^{d e p h}}$. The oscillation frequency $\Omega$ and the dephasing time $\tau^{\text {deph }}$ are given by

$$
\begin{aligned}
\hbar \Omega & =\Delta_{\text {eff }} \cos (\pi \alpha / 2(1-\alpha)), \\
\tau^{d e p h} / \hbar & =\Delta_{\text {eff }}^{-1} \sin ^{-1}(\pi \alpha / 2(1-\alpha)) .
\end{aligned}
$$

These results yield the same quality factor $Q=\Omega \tau^{d e p h}$ as obtained by Lesage and Saleur [29] (see Eqs. (3.155) and (3.159)). However, the aymptotic behaviour given by the algebraic decay of the incoherent part is not correct, as more detailed studies of the interblip correlations show [1]. Concerning the asymptotics of equilibrium correlation functions the NIBA also fails, for it violates the Shiba-relation. 


\section{Appendix B}

\section{Relation of the SBM to the Kondo model}

The Kondo model describes an impurity with spin $1 / 2$ in a conduction band. It interacts with the conduction electrons via an exchange scattering potential. In order to map this problem on the spin-boson model, which we studied in Chapter 3 one has to consider the anisotropic Kondo model, where one deals with different coupling constants for processes which conserve the impurity spin $\left(J_{\|}\right)$, and spin-flip processes $\left(J_{\perp}\right)$. The corresponding Hamiltonian reads

$$
\begin{aligned}
H_{K}= & \sum_{k \sigma} \epsilon_{k} c_{k \sigma}^{\dagger} c_{k \sigma}+J_{\perp} \sum_{k k^{\prime}}\left(c_{k \uparrow}^{\dagger} c_{k^{\prime} \downarrow} S^{-}+c_{k \downarrow}^{\dagger} c_{k^{\prime} \uparrow} S^{+}\right) \\
& +J_{\|} \sum_{k k^{\prime}}\left(c_{k \uparrow}^{\dagger} c_{k^{\prime} \uparrow}-c_{k \downarrow}^{\dagger} c_{k^{\prime} \downarrow}\right) S_{z}-g \mu_{B} h S_{z} .
\end{aligned}
$$

Here $c_{k \sigma}^{\dagger}\left(c_{k \sigma}\right)$ creates (annihilates) a conduction electron with spin $\sigma$ and energy $\epsilon_{k}$. $S_{i}$ ( $i=x, y, z)$ are the impurity spin operators with $S^{ \pm}=S_{x} \pm i S_{y}$. The last term in Eq. (B.1) represents the energy when a local magnetic field $h$ in $z$ direction couples to the impurity. In contrast to this model, in Chapter 5 we investigated the isotropic $\left(J_{\|}=J_{\perp}\right)$ two-lead Kondo model, where the impurity is coupled to two different bands.

The electron-hole excitations have bosonic character. In fact, using bosonization $[91,92]$ the Kondo Hamiltonian in Eq. (B.1) transforms into the spin-boson Hamiltonian given by Eqs. (3.2), (3.5) and (3.6). The parameters are then given by

$$
\begin{aligned}
\Delta / D & =\rho J_{\perp} \cos ^{2}\left(\delta_{K}\right), \\
\alpha & =\left(1-2 \delta_{K} / \pi\right)^{2}, \\
\epsilon & =-g \mu_{B} h
\end{aligned}
$$

where $\rho$ is the constant density of states of the conduction electrons. The scattering phase shift $\delta_{K}$ depends on the applied regularization [93]. For a separable form one obtains $\delta_{K}=\arctan \left(\pi \rho J_{\|} / 4\right)$, whereas else one deals with $\delta_{K}=\pi \rho J_{\|} / 4$. Thus, the universality 
of this mapping is rigorously correct only in the limit

$$
\begin{aligned}
\rho J_{\perp} & =\Delta / D \ll 1, \\
\rho\left|J_{\|}\right| & =|1-\alpha| \ll 1 .
\end{aligned}
$$

The ferromagnetic regime $\rho J_{\|}<0$ corresponds to $\alpha>1$, whereas the antiferromagnetic Kondo model with $\rho J_{\|}>0$ translates into $\alpha<1$. In the antiferromagnetic regime, the Kondo temperature $T_{K}$ sets the temperature scale, below which perturbation theory breaks down. The corresponding characteristic energy scale of the spin-boson model is the renormalized tunnel matrix element $\Delta_{r}$ (see Eq. (3.11)). For $\rho J_{\perp} \ll \rho J_{\|} \ll 1$, which is equivalent to $\Delta / D \ll 1-\alpha \ll 1$, the relation between $T_{K}$ and $\Delta_{r}$ reads

$$
k_{B} T_{K}=\frac{2}{\pi}\left(\frac{1}{2(1-\alpha)}\right)^{1 /(1-\alpha)} \Delta_{r}
$$




\section{Appendix C}

\section{Double vertices in the RTRG}

In Section 3.2 we derived the RG equations for the spin-boson model in the real-time renormalization-group formalism. We applied an approximation by neglecting the double and higher-order vertex objects. Here, we include the double vertex objects within the systematic approach. We again symbolically write $G_{1} \equiv G^{p_{1}}\left(t_{1}\right), A_{1} \equiv A^{p_{1}}\left(t_{1}\right)$ and $B_{1} \equiv B^{p_{1}}\left(t_{1}\right)$. The double vertices are denoted by $G_{12} \equiv G^{p_{1}, p_{2}}\left(t_{1}, t_{2}\right)$ respectively $A_{12} \equiv A^{p_{1}, p_{2}}\left(t_{1}, t_{2}\right), B_{12} \equiv B^{p_{1}, p_{2}}\left(t_{1}, t_{2}\right)$. Henceforth, we choose the time ordering always by $t_{n-1}>t_{n}(n>1)$, and we again use the cross contraction " $\aleph_{7}$ " defined in Eq. (3.47). Note that, here also double vertices may be connected by a cross contraction. Thus, it is important, to which index the contraction refers to. If $t_{1}$ of a double vertex $G_{12}$ is connected only to vertices at time points $t>t_{1}$ and $t_{2}$ only to vertices at $t<t_{2}$, the resulting term may lead to a reducible diagram. We call such a double vertex object $G_{12}^{r}$. (Note that such a configuration leading to reducible diagrams is not possible for a boundary vertex.) Correspondingly, $G_{12}^{i}$ denotes the double vertex, which in any case gives rise to irreducible diagrams. Since only irreducible diagrams contribute to $\Sigma(z)$, we have to distinguish those double vertices. We write

$$
G_{12}=G_{12}^{r}+G_{12}^{i}
$$

The RG equation for the Liouvillian reads

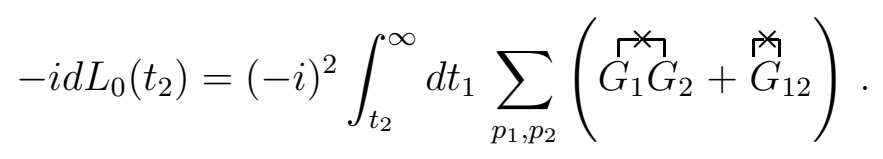

Note that in this appendix we again use $\hbar=1$. Additionally, in the following we omit the factors $(-i)$. Furhermore, neither the time-integrals nor the $p$ sums are written explicitly. By convention those indices, which are denoted by a hat, are identified on the left-hand side and the right-hand side. The remaining indices on the right-hand side correspond to integration variables $t_{i}$ and summation indices $p_{i}$. These indices are connected by a contraction. 
Extending the scheme of Section 3.2 to double vertex objects then yields

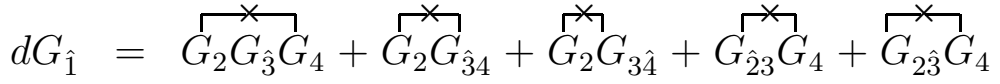

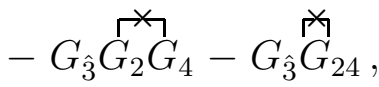

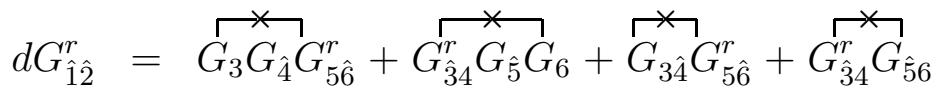

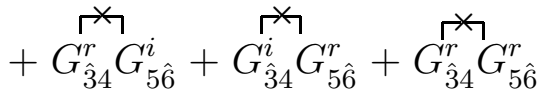

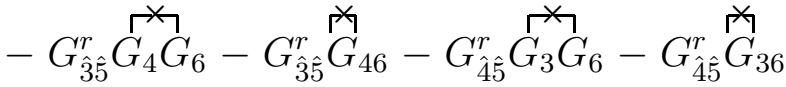

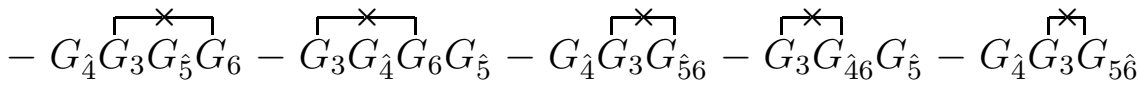

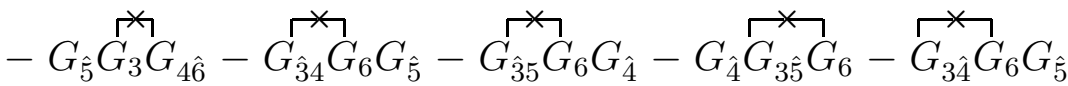

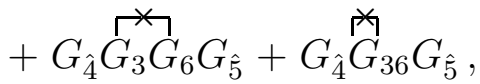

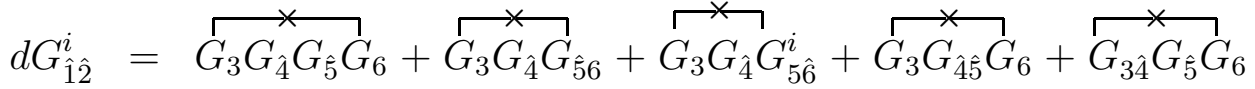

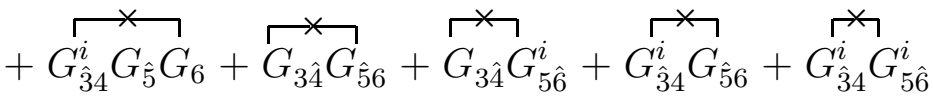

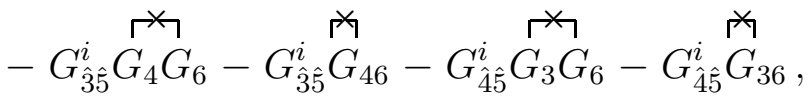

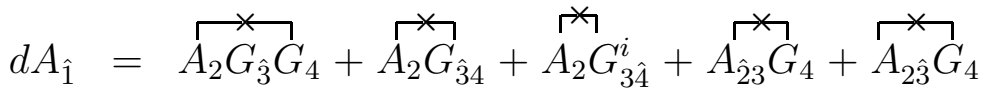

$$
\begin{aligned}
& -A_{\hat{3}}{\stackrel{\text { 盾 }}{G_{2}}}_{4}-A_{\hat{3}} \stackrel{\text { G }}{G}_{24}
\end{aligned}
$$

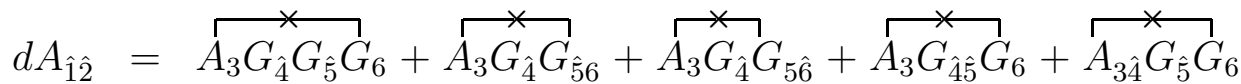

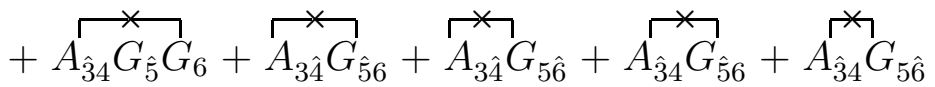

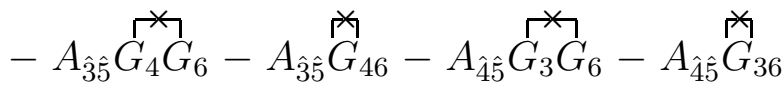

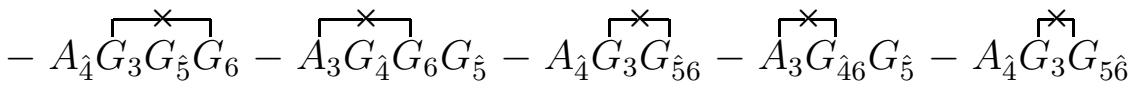

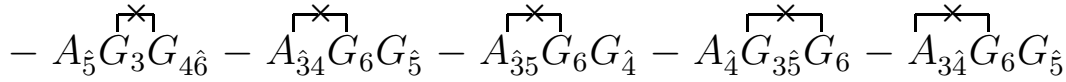

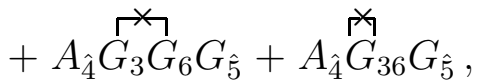




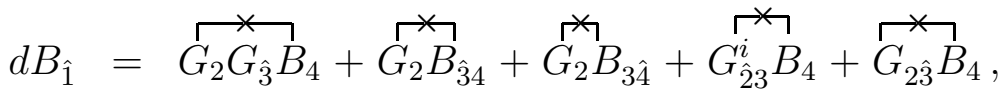

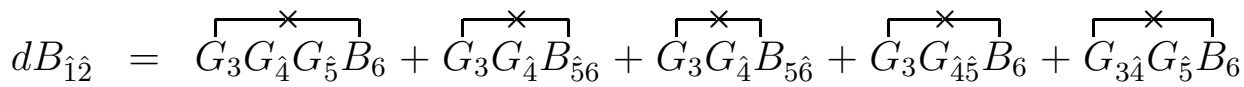

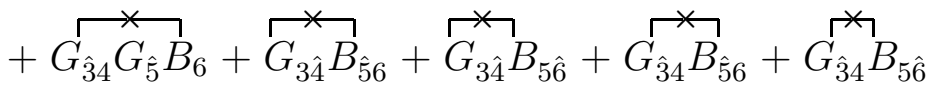

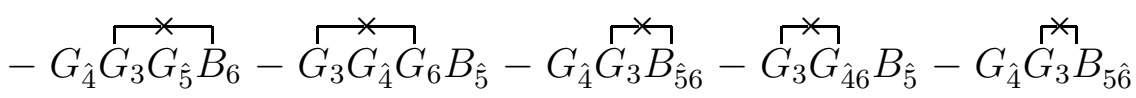

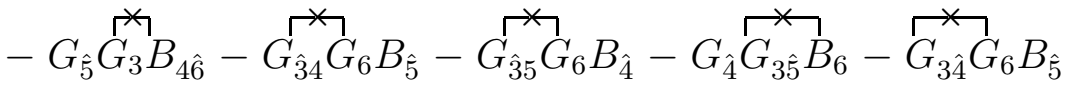

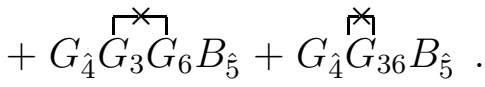

For $d \Sigma(z)$ we obtain

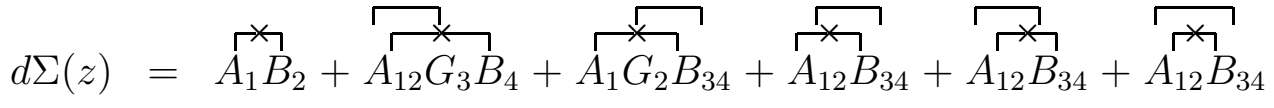

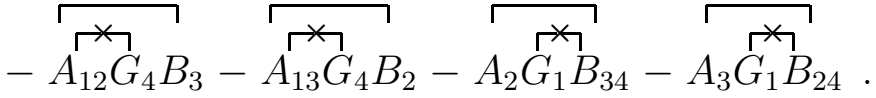

Note that in the above equation there is one integration less than time variables, since the Laplace integral involves only the time difference between the larger time of $A$ and the smaller time of $B$. Furthermore, " $\neg$ " again represents the usual contraction. The correction terms have an additional minus sign, which is accounted for by the signs in Eqs. (C.3) - (C.10).

As pointed out in Section 3.2, the number of terms strongly increases by considering double vertex objects. Furthermore, the RG equations are now integro-differential equations. The reason is a nontrivial time-dependence of the double vertices due to retardation effects. If one uses the interaction picture for the double vertices, one ends up with equations for

$$
\begin{aligned}
& G^{p_{1}, p_{2}}\left(t_{1}-t_{2}\right)=e^{-i L_{0} t_{1}} G^{p_{1}, p_{2}}\left(t_{1}, t_{2}\right) e^{i L_{0} t_{2}} \\
& A^{p_{1}, p_{2}}\left(t_{1}-t_{2}\right)=e^{-i z t_{1}} A^{p_{1}, p_{2}}\left(t_{1}, t_{2}\right) e^{i L_{0} t_{2}} \\
& B^{p_{1}, p_{2}}\left(t_{1}-t_{2}\right)=e^{-i L_{0} t_{1}} B^{p_{1}, p_{2}}\left(t_{1}, t_{2}\right) e^{i z t_{2}} .
\end{aligned}
$$

Thus, the numerical solution of these equations requires much more effort than solving the problem within the single vertex approximation. However, in Chapter 5 we formulate the real-time renormalization-group approach in energy space. Then, the retardation problems are not present, and double vertices become tractable.

Eventually, we note that, as in Section 3.2, the results presented in this appendix are generally valid for any quantum dissipative system. Again for fermionic environments one has to account for additional signs. 



\section{Appendix D}

\section{Keldysh formalism for the TLKM}

In Chapter 5 we studied the running couplings for the two-lead Kondo model (TLKM). However, we restricted ourselves to a study of the effective Hamiltonian for $T=0$. In this appendix we present the RG equations for an analysis on the Keldysh contour. To treat the double vertex objects we again apply the formulation in energy space.

Similarly as in Chapter 3 we define superoperators by

$$
\begin{aligned}
L_{0} O & =\left[H_{0}, O\right]_{-}, \\
G_{\nu, \nu^{\prime}}^{++} O & =g_{\nu, \nu^{\prime}} O \\
G_{\nu, \nu^{\prime}}^{--} O & =-O g_{\nu, \nu^{\prime}}
\end{aligned}
$$

where $O$ is again an usual operator, $H_{0}$ is the impurity Hamiltonian which vanishes initially, and $g_{\nu, \nu^{\prime}}$ is given by Eq. (5.9). Furthermore, initially the superoperators $G_{\nu, \nu^{\prime}}^{+-}$and $G_{\nu, \nu^{\prime}}^{-+}$vanish. We again use the interaction picture analogously to Eq. (5.17):

$$
G_{\nu, \nu^{\prime}, \omega, \omega^{\prime}}^{p p^{\prime}}(t)=e^{i L_{0} t} G_{\nu, \nu^{\prime}, \omega, \omega^{\prime}} e^{-i L_{0} t} e^{-i \eta \omega t} e^{-i \eta^{\prime} \omega^{\prime} t}
$$

Additionally, we introduce

$$
C_{\nu, \nu^{\prime}, \omega, \omega^{\prime}}^{p p^{\prime}}=\frac{1}{2}\left(p G_{\nu, \nu^{\prime}, \omega, \omega^{\prime}}^{p p^{\prime}}-p^{\prime} G_{\nu^{\prime}, \nu, \omega^{\prime}, \omega}^{p^{\prime} p}\right)
$$

For setting up the RG equations we have to choose a cutoff-dependence. In generalization of our definition in Eqs. (5.34) we also include a dependence on the matrix elements of $L_{0}$. The matrix elements are chosen corresponding to those of the external vertices and the contraction vertex, so that again divergencies are avoided. As outlined in Appendix E.2 we may assume a block-diagonal Liouvillian $L_{0}$ with a maximum block size of $(2 \times 2)$. For the eigenvalues of these blocks we use the same notation as Appendix E.2. We define

$$
T_{i \sigma_{i}, k \sigma_{k}, j \sigma_{j}}=\frac{1}{2} \lambda_{i \sigma_{i}}-\lambda_{k \sigma_{k}}+\frac{1}{2} \lambda_{j \sigma_{j}}
$$

where $\lambda_{i \sigma_{i}}$ are the eigenvalues of the blocks as given by Eqs. (E.26) and (E.28). Furthermore, we use the matrix $A_{i i^{\prime} \sigma_{i}}$ defined in Eqs. (E.25) and (E.27). For $L_{0}$ we obtain the 
RG equation

$$
\begin{aligned}
\left(\frac{d L_{0}}{d \omega_{c}}\right)_{i j}= & \sum_{i^{\prime} j^{\prime} k k^{\prime} \sigma_{i} \sigma_{k} \sigma_{j}} \sum_{p_{1} p_{2} p_{3} p_{4}, \nu_{1} \nu_{2}}\left[\frac{1}{2} \int d \omega p_{3} p_{4} \frac{f\left(p_{1} \Delta_{-}\left(\omega_{c}\right)\right) f\left(-p_{2} \omega\right)}{\left(-\omega_{c}-i \operatorname{Im}\left[T_{i \sigma_{i} k \sigma_{k} j \sigma_{j}}\right]\right)}\right. \\
& \times\left(1-\frac{d}{d \omega_{c}} \operatorname{Re}\left[T_{i \sigma_{i} k \sigma_{k} j \sigma_{j}}\right]\right) \\
& \times A_{i i^{\prime} \sigma_{i}}\left(C_{\nu_{1}, \nu_{2}, \Delta_{-}\left(\omega_{c}\right), \omega}^{p_{4} p_{3}}\right)_{i^{\prime} k} A_{k k^{\prime} \sigma_{k}}\left(C_{\nu_{2}, \nu_{1}, \omega, \Delta_{-}\left(\omega_{c}\right)}^{p_{2} p_{1}}\right)_{k^{\prime} j^{\prime}} A_{j^{\prime} j \sigma_{j}} \\
& +\frac{1}{2} \int d \omega p_{3} p_{4} \frac{f\left(p_{1} \omega\right) f\left(-p_{2} \Delta_{+}\left(\omega_{c}\right)\right)}{\left(\omega_{c}-i \operatorname{Im}\left[T_{\left.\left.i \sigma_{i} k \sigma_{k} j \sigma_{j}\right]\right)}\right.\right.} \\
& \times\left(1+\frac{d}{d \omega_{c}} \operatorname{Re}\left[T_{i \sigma_{i} k \sigma_{k} j \sigma_{j}}\right]\right) \\
& \left.\times A_{i i^{\prime} \sigma_{i}}\left(C_{\nu_{2}, \nu_{1}, \omega, \Delta_{+}\left(\omega_{c}\right)}^{p_{4} p_{3}}\right)_{i^{\prime} k} A_{k k^{\prime} \sigma_{k}}\left(C_{\nu_{1}, \nu_{2}, \Delta_{+}\left(\omega_{c}\right), \omega}^{p_{2} p_{1}}\right)_{k^{\prime} j^{\prime}} A_{j^{\prime} j \sigma_{j}}\right] \\
& +\sum_{i^{\prime} j^{\prime} k k^{\prime} \sigma_{i} \sigma_{k} \sigma_{j}} \sum_{p_{1} p_{2} p_{3} p_{4}, \nu_{1} \nu_{2}}\left[\omega_{c} \rightarrow-\omega_{c}\right]
\end{aligned}
$$

with

$$
\Delta_{\eta}\left(\omega_{c}\right)=\omega_{c}+\omega-\mu_{\alpha_{1}}+\mu_{\alpha_{2}}+\eta \operatorname{Re}\left[T_{i \sigma_{i} k \sigma_{k} j \sigma_{j}}\right] .
$$

Here, we considered the case of arbitrary temperature $T$ and used the Fermi-function $f(x)=1 /\left(e^{x / T}+1\right) . \quad \mu_{\alpha}$ is again the chemical potential of the reservoir $\alpha$. The RG equation for $C_{\nu_{1}, \nu_{2}, \omega_{1}, \omega_{2}}^{p_{1} p_{2}}$ reads

$$
\begin{aligned}
& \left(\frac{d C_{\nu_{1}, \nu_{2}, \omega_{1}, \omega_{2}}^{p_{1} p_{2}}}{d \omega_{c}}\right)_{i j}=\sum_{i^{\prime} j^{\prime} k k^{\prime} \sigma_{i} \sigma_{k} \sigma_{j}} \sum_{p_{1}^{\prime} p_{2}^{\prime}, \nu}\left[p_{1} p_{1}^{\prime} \frac{\left.f\left(-p_{2}^{\prime} \Delta_{+}\left(\omega_{c}\right)\right)\right]}{\left(\omega_{c}-i \operatorname{Im}\left[T_{i \sigma_{i} k \sigma_{k} j \sigma_{j}}\right]\right)}\right. \\
& \times\left(1+\frac{d}{d \omega_{c}} \operatorname{Re}\left[T_{i \sigma_{i} k \sigma_{k} j \sigma_{j}}\right]\right) \\
& \times A_{i i^{\prime} \sigma_{i}}\left(C_{\nu_{1}, \nu, \omega_{1}, \Delta_{+}\left(\omega_{c}\right)}^{p_{1} p^{\prime}}\right)_{i^{\prime} k} A_{k k^{\prime} \sigma_{k}}\left(C_{\nu, \nu_{2}, \Delta_{+}\left(\omega_{c}\right), \omega_{2}}^{p_{2}^{\prime} p_{2}}\right)_{k^{\prime} j^{\prime}} A_{j^{\prime} j \sigma_{j}} \\
& -p_{2} p_{2}^{\prime} \frac{f\left(p_{1}^{\prime} \Delta_{-}\left(\omega_{c}\right)\right)}{\left(-\omega_{c}-i \operatorname{Im}\left[T_{i \sigma_{i} k \sigma_{k} j \sigma_{j}}\right]\right)} \\
& \times\left(1-\frac{d}{d \omega_{c}} \operatorname{Re}\left[T_{i \sigma_{i} k \sigma_{k} j \sigma_{j}}\right]\right) \\
& \left.\times A_{i i^{\prime} \sigma_{i}}\left(C_{\nu, \nu_{2}, \Delta_{-}\left(\omega_{c}\right), \omega_{2}}^{p_{2}^{\prime} p_{2}}\right)_{i^{\prime} k} A_{k k^{\prime} \sigma_{k}}\left(C_{\nu_{1}, \nu, \omega_{1}, \Delta_{-}\left(\omega_{c}\right)}^{p_{1} p^{\prime}}\right)_{k^{\prime} j^{\prime}} A_{j^{\prime} j \sigma_{j}}\right] \\
& +\sum_{i^{\prime} j^{\prime} k k^{\prime} \sigma_{i} \sigma_{k} \sigma_{j}} \sum_{p_{1}^{\prime} p_{2}^{\prime}, \nu}\left[\omega_{c} \rightarrow-\omega_{c}\right]
\end{aligned}
$$


with

$$
\Delta_{\eta}\left(\omega_{c}\right)=\omega_{c}+\bar{\omega}+\bar{V}_{\alpha}+\eta \operatorname{Re}\left[T_{i \sigma_{i} k \sigma_{k} j \sigma_{j}}\right] .
$$

The parameters $\bar{\omega}$ and $\bar{V}_{\alpha}$ are defined in Eqs. (5.30) and (5.31).

The above equations fulfill the symmetry of spin inversion on the impurity and

$$
C_{\nu_{1}, \nu_{2}, \omega_{1}, \omega_{2}}^{p_{1} p_{2}}=-C_{\bar{\nu}_{2}, \bar{\nu}_{1},-\omega_{2},-\omega_{1}}^{p_{2} p_{1}}
$$

where $\bar{\nu}=(\eta, \bar{\alpha}, \sigma)$ corresponds to the opposite reservoir $\bar{\alpha}$ of the band $\alpha$.

In the above RG equations the matrix elements of the Liouvillian may act as a cutoff in a similar way as the voltage $V$ does in Section 5.3. By accounting for the Liouvillian we also consider rates here, i.e. by solving the RG equations the question can be examined, if there is two-channel physics in case of the two-lead Kondo model or if it is destroyed by the rates. Note that observables can be calculated by considering kernels $\Sigma$ (as in Chapter 3) or $\Sigma_{I}$ (as in Chapter 4). Their RG equations are given analogously to Eq. (D.6).

However, a numerical solution of Eqs. (D.6) and (D.8) turns out to require too much numerical effort since we deal with frequency-dependent $(4 \times 4)$-matrices. As already pointed out in Section 5.4 the numerical solution for only 10 frequencies on a PIII (500MHz)-computer requires about 100 days. 



\section{Appendix E}

\section{Numerical methods}

\section{E.1 Ordinary differential equations}

Renormalization-group approaches typically require the solution of an initial value problem for ordinary differential equations. In this thesis we solved these equations using numerical methods. In the following, we give a description of the applied algorithm.

There are many different methods for the numerical solution of initial value problems for ordinary differential equations. Among them the Runge-Kutta method is used very often. It provides a stable and efficient algorithm for most problems, if one does not require too high accuracy [94]. Regarding our problems it turned out that this method performs very well.

In general, the initial value problem is given by

$$
\begin{aligned}
\frac{d y_{i}}{d x}(x) & =f_{i}\left(x, y_{1}, \ldots, y_{N}\right) \\
y_{i}\left(x_{0}\right) & =y_{0 i}, \quad i=1, \ldots, N
\end{aligned}
$$

The Runge-Kutta algorithm is based on the Euler method, where one integrates the differential equations stepwise with a stepsize $h$ :

$$
\vec{y}(x+h)=\vec{y}(x)+h \vec{f}(x, \vec{y})+\mathcal{O}\left(h^{2}\right) .
$$

Here we used the vector notation for the $N$ components $y_{i}$ and $f_{i}$. Conventially one calls a method $n$th order if its error term is $\mathcal{O}\left(h^{n+1}\right)$. Thus, this simple Euler scheme is a firstorder method. It is not of any practical use because of low accuracy and stability problems. The reason is that at each integration step only the information of the derivatives at the original point is used. Therefore, the method can be improved by also using the derivative information at some intermediate point(s), e.g. the midpoint of the step interval. With the right combination of these derivatives one may eliminate the error in higher orders. One very often used algorithm is the fourth-order Runge-Kutta method, which needs four derivative calculations per step. However, we use a Runge-Kutta algorithm where we need six derivative calculations. The advantage of this additional effort is that we may 


\begin{tabular}{|c|c|c|c|c|c|c|c|c|}
\hline & & & $b_{i j}$ & & & $a_{i}$ & $c_{i}^{(4)}$ & $c_{i}^{(5)}$ \\
\hline$i$ & 1 & 2 & 3 & 4 & 5 & & & \\
\hline 1 & & & & & & & $\frac{2825}{27648}$ & $\frac{37}{378}$ \\
\hline 2 & $\frac{1}{5}$ & & & & & $\frac{1}{5}$ & 0 & 0 \\
\hline 3 & $\frac{3}{40}$ & $\frac{9}{40}$ & & & & $\frac{3}{10}$ & $\frac{18575}{48384}$ & $\frac{250}{621}$ \\
\hline 4 & $\frac{3}{10}$ & $-\frac{9}{10}$ & $\frac{6}{5}$ & & & $\frac{3}{5}$ & $\frac{13525}{55296}$ & $\frac{125}{594}$ \\
\hline 5 & $-\frac{11}{54}$ & $\frac{5}{2}$ & $-\frac{70}{27}$ & $\frac{35}{27}$ & & 1 & $\frac{277}{14336}$ & 0 \\
\hline 6 & $\frac{1631}{55296}$ & $\frac{175}{512}$ & $\frac{575}{13824}$ & $\frac{44275}{110592}$ & $\frac{253}{4096}$ & $\frac{7}{8}$ & $\frac{1}{4}$ & $\frac{512}{1771}$ \\
\hline
\end{tabular}

Table E.1: Cash-Karp parameters for embedded Runge-Kutta method.

combine these derivatives in two different ways, one resulting in a fourth-order method, Eq. (E.4), the other corresponding to a fifth-order algorithm, Eq. (E.5).

$$
\begin{aligned}
\vec{k}_{1} & =h \vec{f}(x, \vec{y}), \\
\vec{k}_{i} & =h \vec{f}\left(x+a_{i} h, \vec{y}+\sum_{j=1}^{i-1} b_{i j} \vec{k}_{j}\right) \quad, \quad i=2, \ldots, 6 \\
\vec{y}^{(4)}(x+h) & =\vec{y}(x)+c_{1}^{(4)} \vec{k}_{1}+c_{2}^{(4)} \vec{k}_{2}+c_{3}^{(4)} \vec{k}_{3}+c_{4}^{(4)} \vec{k}_{4}+c_{5}^{(4)} \vec{k}_{5}+c_{6}^{(4)} \vec{k}_{6}, \\
\vec{y}(x+h) & =\vec{y}^{(4)}(x+h)+\mathcal{O}\left(h^{5}\right), \\
\vec{y}^{(5)}(x+h) & =\vec{y}(x)+c_{1}^{(5)} \vec{k}_{1}+c_{2}^{(5)} \vec{k}_{2}+c_{3}^{(5)} \vec{k}_{3}+c_{4}^{(5)} \vec{k}_{4}+c_{5}^{(5)} \vec{k}_{5}+c_{6}^{(5)} \vec{k}_{6}, \\
\vec{y}(x+h) & =\vec{y}^{(5)}(x+h)+\mathcal{O}\left(h^{6}\right) .
\end{aligned}
$$

The parameters in Tab. E.1 have been found by Cash and Karp [95]. This type of equations is called an embedded Runge-Kutta formula. By comparing both results one obtains an error estimate $\Delta_{i}=\left|y_{i}^{(4)}-y_{i}^{(5)}\right|$ of the fourth-order formula. Using this error information one can then adjust the stepsize to keep a given accuracy $\epsilon_{i}$. As the error scales with $h^{5}$ the adjusted stepsize $h_{a}$ is given by

$$
h_{a}=h \max _{1 \leq i \leq N}\left(\frac{\epsilon_{i}}{\Delta_{i}}\right)^{0.2}
$$

If $h_{a}<h$, the step is rejected and repeated using $h_{a}$ as stepsize, whereas if $h_{a}>h$, the step is accepted and $h_{a}$ is used in the next step. In our numerical integrations we used a relative tolerance $T O L \sim 10^{-4}$ where $T O L=\frac{\epsilon_{i}}{y_{i}}$. For more details on the adaptive stepsize control, see Ref. [94]. 


\section{E.2 Exponentials of a matrix in differential equations}

If the real-time renormalization-group approach is used for the Keldysh contour (as in Chapters 3 and 4), one deals with ordinary differential equations for $N(n \times n)$-matrices $M_{i}$ with respect to $t_{c}$ :

$$
\frac{d M_{i}}{d t_{c}}=f\left(t_{c}, M_{1}, \ldots, M_{N}\right) \quad, \quad 1 \leq i \leq N .
$$

The function on the right-hand side includes exponentials of the matrix $M_{1}=L_{0}$. Thus, for a numerical solution we introduce a transformation $U$ such that

$$
U L_{0} U^{-1}=\left(\begin{array}{ccccc}
l_{1} & & & & \\
& \cdot & & 0 & \\
& & \cdot & & \\
& 0 & & \cdot & \\
& & & & l_{m}
\end{array}\right) \quad, \quad m \leq n .
$$

Here, the $l_{k}(1 \leq k \leq m)$ are $(1 \times 1)$ - or $(2 \times 2)$-matrices. In the following we write $\tilde{M}_{i}=U M_{i} U^{-1}$. For $t_{c}=0$ the transformation $U$ has to be calculated for the initial value of $L_{0}$. To obtain the $t_{c}$ flow of $U$ we first note that

$$
\frac{d \tilde{M}_{i}}{d t_{c}}=\left[E, \tilde{M}_{i}\right]+U \frac{d M_{i}}{d t_{c}} U^{-1} \quad, \quad 1 \leq i \leq N
$$

where $E$ is given by

$$
E=\frac{d U}{d t_{c}} U^{-1}
$$

$E$ shall then be determined such that the block structure of $\tilde{L}_{0}$ in Eq. (E.8) is retained. If the indices $i$ and $j$ correspond to different blocks, we have to fulfill

$$
S_{i j}+\left[E, \tilde{L}_{0}\right]_{i j}=0
$$

where we have written

$$
S=U \frac{d L_{0}}{d t_{c}} U^{-1}
$$

For the case, where both $i$ and $j$ correspond to $(1 \times 1)$-blocks this yields

$$
E_{i j}=\frac{S_{i j}}{\left(\tilde{L}_{0}\right)_{i i}-\left(\tilde{L}_{0}\right)_{j j}} \quad \text { for } \quad\left(\tilde{L}_{0}\right)_{i i} \neq\left(\tilde{L}_{0}\right)_{j j}
$$

If $\left(\tilde{L}_{0}\right)_{i i} \approx\left(\tilde{L}_{0}\right)_{j j}$, i.e. for the crossing of two eigenvalues of $\tilde{L}_{0}$, we interprete them as a $(2 \times 2)$-block. If $i$ corresponds to a $(1 \times 1)$-block, whereas $j$ is an element of a 
$(2 \times 2)$-block, Eq. (E.11) leads to

$$
\begin{aligned}
& E_{i j}= \frac{\left(\left(\tilde{L}_{0}\right)_{i i}-\left(\tilde{L}_{0}\right)_{\bar{j} \bar{j}}\right) S_{i j}+\left(\tilde{L}_{0}\right)_{\bar{j} j} S_{i \bar{j}}}{\left(\left(\tilde{L}_{0}\right)_{i i}-\left(\tilde{L}_{0}\right)_{j j}\right)\left(\left(\tilde{L}_{0}\right)_{i i}-\left(\tilde{L}_{0}\right)_{\bar{j} \bar{j}}\right)-\left(\tilde{L}_{0}\right)_{\bar{j} j}\left(\tilde{L}_{0}\right)_{j \bar{j}}}, \\
& E_{j i}=--\frac{\left(\left(\tilde{L}_{0}\right)_{i i}-\left(\tilde{L}_{0}\right)_{\bar{j} \bar{j}}\right) S_{j i}+\left(\tilde{L}_{0}\right)_{j \bar{j}} S_{\bar{j} i}}{\left(\left(\tilde{L}_{0}\right)_{i i}-\left(\tilde{L}_{0}\right)_{j j}\right)\left(\left(\tilde{L}_{0}\right)_{i i}-\left(\tilde{L}_{0}\right)_{\bar{j} \bar{j}}\right)-\left(\tilde{L}_{0}\right)_{\bar{j} j}\left(\tilde{L}_{0}\right)_{j \bar{j}}} \\
& \text { for } \quad\left(\left(\tilde{L}_{0}\right)_{i i}-\left(\tilde{L}_{0}\right)_{j j}\right)\left(\left(\tilde{L}_{0}\right)_{i i}-\left(\tilde{L}_{0}\right)_{\bar{j} \bar{j}}\right)-\left(\tilde{L}_{0}\right)_{\bar{j} j}\left(\tilde{L}_{0}\right)_{j \bar{j}} \neq 0 .
\end{aligned}
$$

Here, the two indices of the $(2 \times 2)$-block are denoted by $j$ and $\bar{j}$. The case

$$
\left(\left(\tilde{L}_{0}\right)_{i i}-\left(\tilde{L}_{0}\right)_{j j}\right)\left(\left(\tilde{L}_{0}\right)_{i i}-\left(\tilde{L}_{0}\right)_{\bar{j} \bar{j}}\right)-\left(\tilde{L}_{0}\right)_{\bar{j} j}\left(\tilde{L}_{0}\right)_{j \bar{j}} \approx 0
$$

corresponds to the situation, where $\left(\tilde{L}_{0}\right)_{i i}$ crosses one eigenvalue of the $(2 \times 2)$-block. Whenever a $(2 \times 2)$-block involves two different eigenvalues, we therefore stop the $t_{c}$ integration, diagonalize that block, consider it as two $(1 \times 1)$-blocks and resume the integration with a modified transformation $U$. Thus, we may use the above equations, unless three eigenvalues of $\tilde{L}_{0}$ cross each other around one value of $t_{c}$. If $i$ and $j$ correspond to two different $(2 \times 2)$-blocks (with the indices $i$ and $\bar{i}$ respectively $j$ and $\bar{j}$ ), we obtain from Eq. (E.11)

$$
\left(\begin{array}{c}
E_{i j} \\
E_{i \bar{j}} \\
E_{\bar{i} j} \\
E_{\overline{i j}}
\end{array}\right)=T^{-1}\left(\begin{array}{c}
S_{i j} \\
S_{i \bar{j}} \\
S_{\overline{i j}} \\
S_{\overline{i j}}
\end{array}\right)
$$

where the matrix $T$ is given by

$$
T=\left(\begin{array}{cccc}
\left(\tilde{L}_{0}\right)_{i i}-\left(\tilde{L}_{0}\right)_{j j} & -\left(\tilde{L}_{0}\right)_{\bar{j} j} & \left(\tilde{L}_{0}\right)_{i \bar{i}} & 0 \\
-\left(\tilde{L}_{0}\right)_{j \bar{j}} & \left(\tilde{L}_{0}\right)_{i i}-\left(\tilde{L}_{0}\right)_{\bar{j} \bar{j}} & 0 & \left(\tilde{L}_{0}\right)_{i \bar{i}} \\
\left(\tilde{L}_{0}\right)_{\bar{i} i} & 0 & \left(\tilde{L}_{0}\right)_{\overline{i i}}-\left(\tilde{L}_{0}\right)_{j j} & -\left(\tilde{L}_{0}\right)_{\bar{j} j} \\
0 & \left(\tilde{L}_{0}\right)_{\bar{i} i} & -\left(\tilde{L}_{0}\right)_{j \bar{j}} & \left(\tilde{L}_{0}\right)_{\overline{i i}}-\left(\tilde{L}_{0}\right)_{\bar{j} \bar{j}}
\end{array}\right)
$$

As we diagonalize $(2 \times 2)$-blocks with two different eigenvalues, the inverse $T^{-1}$ does exist, unless four eigenvalues of $\tilde{L}_{0}$ are approximately equal for one $t_{c}$. For the case, where the indices $i$ and $j$ belong to the same block, we set $E_{i j}=0$. Thus, $E$ is completely determined and Eq. (E.10) leads to the additional differential equation

$$
\frac{d U}{d t_{c}}=E U
$$


The above scheme now only requires the evaluation of exponentials of the form

$$
U e^{c L_{0}} U^{-1}=\left(\begin{array}{ccccc}
e^{c l_{1}} & & & & \\
& \cdot & & 0 & \\
& & \cdot & & \\
& 0 & & \cdot & \\
& & & e^{c l_{m}}
\end{array}\right)
$$

where $c$ is a complex number. The calculation of $\exp \left(c l_{k}\right)$ is trivial in case of $(1 \times 1)$ blocks. The exponential of a $(2 \times 2)$-matrix can be written as

$$
e^{c l_{k}}=\sum_{\sigma= \pm} A_{\sigma}^{(2)} e^{c \lambda_{k, \sigma}^{(2)}} .
$$

With the parameters

$$
\begin{aligned}
a_{k}^{(2)} & =\frac{1}{2}\left(\left(l_{k}\right)_{11}+\left(l_{k}\right)_{22}\right) \\
b_{k}^{(2)} & =\left(\frac{1}{4}\left(\left(l_{k}\right)_{11}-\left(l_{k}\right)_{22}\right)^{2}+\left(l_{k}\right)_{12}\left(l_{k}\right)_{21}\right)^{\frac{1}{2}}
\end{aligned}
$$

the matrix $A_{k, \sigma}^{(2)}$ and the eigenvalues $\lambda_{k, \sigma}^{(2)}$ of $l_{k}$ are given by

$$
\begin{aligned}
A_{k, \sigma}^{(2)} & =\frac{\sigma}{2 b_{k}^{(2)}}\left(l_{k}-\lambda_{k,-\sigma}^{(2)} \mathbb{1}\right) \quad \text { for } \quad b_{k}^{(2)} \neq 0, \\
\lambda_{k, \sigma}^{(2)} & =a_{k}^{(2)}+\sigma b_{k}^{(2)} .
\end{aligned}
$$

From the above considerations for $(1 \times 1)$ - or $(2 \times 2)$-matrices one readily obtains

$$
\left(U e^{c L_{0}} U^{-1}\right)_{i j}=\sum_{\sigma= \pm} A_{i j, \sigma} e^{c \lambda_{i, \sigma}}
$$

Here, $A_{i j, \sigma}=0$ holds, if the indices $i$ and $j$ correspond to different blocks. Trivially, if $i=j$ corresponds to a $(1 \times 1)$-block, we have to set

$$
\begin{aligned}
A_{i j, \sigma} & =\delta_{i j} / 2 \\
\lambda_{i, \sigma} & =\left(\tilde{L}_{0}\right)_{i i} .
\end{aligned}
$$

Eventually, if the indices $i$ and $j$ correspond to a $(2 \times 2)$-block, $A_{i j, \sigma}$ and $\lambda_{i, \sigma}$ are given by

$$
\begin{aligned}
A_{i j, \sigma} & =\frac{\sigma}{2 b_{i}}\left(\left(\tilde{L}_{0}\right)_{i j}-\lambda_{i,-\sigma} \delta_{i j}\right) \quad \text { for } \quad b_{i} \neq 0, \\
\lambda_{i, \sigma} & =a_{i}+\sigma b_{i} .
\end{aligned}
$$


Here we used the parameters

$$
\begin{aligned}
a_{i} & =\frac{1}{2}\left(\left(\tilde{L}_{0}\right)_{i i}+\left(\tilde{L}_{0}\right)_{\overline{i i}}\right) \\
b_{i} & =\left(\frac{1}{4}\left(\left(\tilde{L}_{0}\right)_{i i}-\left(\tilde{L}_{0}\right)_{\bar{i} i}\right)^{2}+\left(\tilde{L}_{0}\right)_{i \bar{i}}\left(\tilde{L}_{0}\right)_{\bar{i} i}\right)^{\frac{1}{2}},
\end{aligned}
$$

where we again denote the two indices of that $(2 \times 2)$-block by $i$ and $\bar{i}$. We still have to consider the case where $b_{i} \approx 0$. Generally, we have to calculate products of the matrix elements in Eq. (E.24). Suppose that the indices $i_{1}, \ldots, i_{n}$ correspond to $(2 \times 2)$-blocks with

$$
b_{i_{1}} \approx \cdots \approx b_{i_{n}} \ll 1
$$

The products of the form

$$
P=\sum_{\sigma_{i_{1}}, \ldots, \sigma_{i_{n}}} A_{i_{1} j_{1}, \sigma_{i_{1}}} \cdots A_{i_{n} j_{n}, \sigma_{i_{n}}} f\left(\lambda_{i_{1}, \sigma_{i_{1}}}, \ldots, \lambda_{i_{n}, \sigma_{i_{n}}}\right)
$$

can then be determined by expanding the function $f$. One obtains

$$
\begin{aligned}
P= & \sum_{\sigma_{i_{1}}, \ldots, \sigma_{i_{n}}}\left\{f\left(a_{i_{1}}, \ldots, a_{i_{n}}\right)\right. \\
+ & \sum_{j=1}^{\infty} \frac{1}{j !} \sum_{k_{1}, \ldots, k_{j} \in\left\{i_{1}, \ldots, i_{n}\right\}} \sigma_{k_{1}} \cdots \sigma_{k_{j}} b_{k_{1}} \cdots b_{k_{j}} \frac{\partial^{j}}{\partial \lambda_{k_{1}, \sigma_{k_{1}}} \cdots \partial \lambda_{k_{j}, \sigma_{k_{j}}}} f\left(a_{i_{1}}, \ldots, a_{i_{n}}\right) \\
& \times\left(\sigma_{i_{1}} \frac{\left(\left(\tilde{L}_{0}\right)_{i_{1} j_{1}}-a_{i_{1}} \delta_{i_{1} j_{1}}\right)}{2 b_{i_{1}}}+\frac{\delta_{i_{1} j_{1}}}{2}\right) \cdots \\
& \left.\ldots \times\left(\sigma_{i_{n}} \frac{\left(\left(\tilde{L}_{0}\right)_{i_{n} j_{n}}-a_{i_{n}} \delta_{i_{n} j_{n}}\right)}{2 b_{i_{n}}}+\frac{\delta_{i_{n} j_{n}}}{2}\right)\right\} .
\end{aligned}
$$

Thus, we may write

$$
P \approx \sum_{\sigma_{i_{1}}, \ldots, \sigma_{i_{n}}} B_{i_{1} j_{1}, \sigma_{i_{1}}} \cdots B_{i_{n} j_{n}, \sigma_{i_{n}}} g_{\sigma_{i_{1}}, \ldots, \sigma_{i_{n}}}\left(a_{i_{1}}, \ldots, a_{i_{n}}\right),
$$

where we introduced

$$
\begin{aligned}
B_{i_{s} j_{s},+} & =\delta_{i_{s} j_{s}} \\
B_{i_{s} j_{s},-} & =\left(\tilde{L}_{0}\right)_{i_{s} j_{s}}-a_{i_{s}} \delta_{i_{s} j_{s}} \quad, \quad 1 \leq s \leq n, \\
g_{\sigma_{i_{1}}, \ldots, \sigma_{i_{n}}}\left(a_{i_{1}}, \ldots, a_{i_{n}}\right) & =\frac{1}{j !} \frac{\partial^{j}}{\partial \lambda_{k_{1}, \sigma_{k_{1}}} \cdots \partial \lambda_{k_{j}, \sigma_{k_{j}}}} f\left(a_{i_{1}}, \ldots, a_{i_{n}}\right) \\
\text { for } k_{1}, \ldots, k_{j} & \in\left\{i_{1}, \ldots, i_{n}\right\} \quad \text { and } \sigma_{k_{1}}=\cdots=\sigma_{k_{j}}=-.
\end{aligned}
$$


Note that our result for $b_{i_{1}} \approx \cdots \approx b_{i_{n}} \ll 1$ in Eq. (E.32) is regular for $b_{i_{1}}=\cdots=$ $b_{i_{n}}=0$. Therefore, the prescriptions, which we have developed in this appendix, solve the problem of the occurence of exponential functions. Only if more than two eigenvalues of $\tilde{L}_{0}$ are the same for one $t_{c}$, we obtain a divergence for the matrix $E_{i j}$. This is not the case for the problems studied in Chapters 3 and 4.

\section{E.3 Fourier transforms}

The real-time renormalization-group approach is formulated for the Laplace tranform of the reduced density matrix $p(z)$. From the numerical data for $p(z)$ the time-dependent reduced density matrix can be calculated by a Fourier transform (see Subsection 3.4.3). In the following, we outline the algorithm we used for that problem.

We have to perform the integral

$$
\tilde{f}(t)=\int_{-\infty}^{\infty} d x f(x) e^{-i x t}
$$

where $f(x)$ follows from the RG procedure. For large enough $d$ this integral is approximately given by

$$
\tilde{f}(t) \approx \int_{-d}^{d} d x f(x) e^{-i x t}-\frac{f(-d) e^{i d t}}{i t}+\frac{f(d) e^{-i d t}}{i t} .
$$

The error is controlled by calculating $\tilde{f}(t)$ for different cutoffs $d$. For the numerical evaluation of the integral in Eq. (E.35) we have to discretize $f(x)$. It turns out that there are subintervals in $[-d, d]$ with quite different scales, which $f(x)$ varies on. Therefore, we split the integral into these subintervals applying an adequate discretization of $f(x)$ for each. Thus, we have to consider integrals of the form

$$
I(t)=\int_{a}^{b} d x f(x) e^{-i x t}
$$

For the numerical evaluation of Eq. (E.36) we set the discretization $\Delta x=\frac{b-a}{M}$ with $M \in \mathbb{N}$. Then $I(t)$ is a periodic function with the period $2 \bar{t}$, where $\bar{t}=\pi / \Delta x$ is the time corresponding to the Nyquist critical frequency [94]. As the times $t_{1}$ and $t_{2}=t_{1}+2 \bar{t}$ give the same discretized $f(x)$, the value of $I(t)$ for $t>\bar{t}$ is falsely translated ("aliased") into the interval $[-\bar{t}, \bar{t}]$. Therefore, given a certain discretization we may only determine $I(t)$ for $t<\bar{t}$.

With $x_{j}=a+j \Delta x$ for $0 \leq j \leq M$ the integration then requires the evaluation of $f$ at the points $x_{j}$. For large $\left|x_{j}\right|$ it is possible to fit the decay of $f(x)$ algebraically, so that in this case we need not calculate $f\left(x_{j}\right)$ from the RG equations.

A straightforward application of a standard integration method, such as the extended trapezoidal rule, is not possible though. As in case of the RG equations for the polaron in Chapter 2 the oscillatory nature of the integral would lead to large errors, for the integrand 
oscillates with a frequency $t<\bar{t}$, i.e. the parameter determining the error, $\Delta x t$, will be as large as $\pi$. As in Chapter 2 we use interpolation to overcome these difficulties. Using a piecewise polynomial interpolation scheme, $f(x)$ is of the general form

$$
f(x) \approx \sum_{j=0}^{M} f\left(x_{j}\right) \psi\left(\frac{x-x_{j}}{\Delta x}\right)+\sum_{j=\text { endpoints }} f\left(x_{j}\right) \phi_{j}\left(\frac{x-x_{j}}{\Delta x}\right)
$$

where the functions $\psi$ and $\phi_{j}$ depend on the applied interpolation scheme. Inserting this expression in Eq. (E.36) yields

$$
I(t) \approx \Delta x e^{-i a t}\left(W(\Delta x t) \sum_{j=0}^{M} f\left(x_{j}\right) e^{-i j \Delta x t}+\sum_{j=\text { endpoints }} f\left(x_{j}\right) \alpha_{j}(\Delta x t)\right)
$$

with the definitions

$$
\begin{aligned}
& W(y)=\int_{-\infty}^{\infty} d s e^{-i y s} \psi(s) \\
& \alpha_{j}(y)=\int_{-\infty}^{\infty} d s e^{-i y s} \phi_{j}(s-j) .
\end{aligned}
$$

For a given interpolation scheme we may evaluate the above functions analytically. The sum in Eq. (E.38) corresponds to a discrete Fourier transform. In view of a finer sampling in time space we introduce $N>M, N \in \mathbb{N}$. Setting $f\left(x_{j}\right)=0$ for $M<j<N$ we then extend the sum, so that $j$ takes the range $0 \leq j \leq N-1$. Thereby we are able to produce an output for $t_{n}=2 \pi n / N \Delta x$ with $0 \leq n \leq N / 2-1$. $N$ is chosen as an integer power of 2 , because then, we may easily apply the fast Fourier transform to perform the sum. This standard algorithm reduces the computational effort from $\mathcal{O}\left(N^{2}\right)$ to $\mathcal{O}(N \log N)$ by rewriting a discrete Fourier transform of length $N$ as two discrete Fourier transforms of length $N / 2$ [94].

Using a cubic interpolation scheme in Eq. (E.37) we finally obtain

$$
\begin{aligned}
I\left(t_{n}\right)= & \Delta x e^{-i a t_{n}}\left(W\left(\Delta x t_{n}\right) \sum_{j=0}^{N-1} f\left(x_{j}\right) e^{-2 \pi i j n / N}\right. \\
& \left.+\sum_{j=0}^{3}\left(\alpha_{j}\left(\Delta x t_{n}\right) f\left(x_{j}\right)+e^{-i(b-a) t_{n}} \alpha_{j}^{*}\left(\Delta x t_{n}\right) f\left(x_{M-j}\right)\right)\right)
\end{aligned}
$$


with the functions

$$
\begin{aligned}
W(y)= & \frac{6+y^{2}}{3 y^{4}}(3-4 \cos y+\cos (2 y)) \\
\alpha_{0}(y)= & \frac{-42+5 y^{2}+\left(6+y^{2}\right)(8 \cos y-\cos (2 y))}{6 y^{4}} \\
& -i \frac{-12 y+6 y^{3}+\left(6+y^{2}\right) \sin (2 y)}{6 y^{4}}, \\
\alpha_{1}(y)= & \frac{14\left(3-y^{2}\right)-7\left(6+y^{2}\right) \cos y}{6 y^{4}}-i \frac{30 y-5\left(6+y^{2}\right) \sin y}{6 y^{4}}, \\
\alpha_{2}(y)= & \frac{-4\left(3-y^{2}\right)+2\left(6+y^{2}\right) \cos y}{3 y^{4}}-i \frac{-12 y+2\left(6+y^{2}\right) \sin y}{3 y^{4}}, \\
\alpha_{3}(y)= & \frac{2\left(3-y^{2}\right)-\left(6+y^{2}\right) \cos y}{6 y^{4}}-i \frac{6 y-\left(6+y^{2}\right) \sin y}{6 y^{4}} .
\end{aligned}
$$

To calculate $I(t)$ for all $0<t<\bar{t}$ we perform polynomial interpolation on the spectrum of the discrete Fourier transform. This interpolation, however, is only accurate for a large "oversampling" $(N \gg M)[94]$. 



\section{Bibliography}

[1] U. Weiss, Quantum Dissipative Systems (World Scientific, Singapore, 2nd edition, 2000).

[2] R. P. Feynman and A. R. Hibbs, Quantum Mechanics and Path Integrals (Mc GrawHill, New York, 1965).

[3] F. Wegner, Ann. Physik (Leipzig) 3, 77 (1994).

[4] P. W. Anderson, G. Yuval, D. R. Hamann, Phys. Rev. B 1, 4464 (1970).

[5] K. G. Wilson, Rev. Mod. Phys. 47, 773 (1975).

[6] T. A. Costi, C. Kieffer, Phys. Rev. Lett.76, 1683 (1996).

[7] C. J. Halboth, W. Metzner, Phys. Rev. B 61, 7364 (2000).

[8] V. Meden, W. Metzner, U. Schollwöck, and K. Schönhammer, cond-mat/0104336

[9] V. Meden, W. Metzner, U. Schollwöck, and K. Schönhammer, cond-mat/0109013

[10] W. Hofstetter, H. Schoeller, cond-mat/0108359

[11] W. Hofstetter, J. König, H. Schoeller, Phys. Rev. Lett. 87, 156803 (2001).

[12] H. Schoeller, in Low-Dimensional Systems, edited by T. Brandes (Springer, Berlin, 1999), p. 137.

[13] J. König, H. Schoeller, Phys. Rev. Lett. 81, 3511 (1998).

[14] H. Schoeller, J. König, Phys. Rev. Lett. 84, 3686 (2000).

[15] H. Fröhlich, Adv. Phys. 3, 325 (1954).

[16] J. Sak, Phys. Rev. B 6, 3981 (1972).

[17] S. Das Sarma, Phys. Rev. B 27, 2590 (1983).

[18] F. M. Peeters, Wu Xiaoguang and J. T. Devreese, Phys. Rev. B 33, 3926 (1986).

[19] F. M. Peeters, M. A. Smondyrev, Phys. Rev. B 43, 4920 (1991). 
[20] V. Meden, K. Schönhammer, and O. Gunnarson, Phys. Rev. B 50, 11179 (1994).

[21] T. D. Lee, F. E. Low and D. Pines, Phys. Rev. 90, 297 (1953).

[22] T. D. Lee, D. Pines, Phys. Rev. 92, 883 (1953).

[23] R. P. Feynman, Phys. Rev. 97, 660 (1955).

[24] M. Keil, H. Schoeller, Phys. Rev. B 62, 2990 (2000).

[25] M. Keil, H. Schoeller, Phys. Rev. B 63, 180302(R) (2001).

[26] M. Keil, H. Schoeller, Chemical Physics 268, 11-20 (2001).

[27] A. J. Leggett, S. Chakravarty, A. T. Dorsey, M. P. A. Fisher, A. Garg, W. Zwerger, Rev. Mod. Phys. 59, 1 (1987).

[28] T. A. Costi, G. Zarand, Phys. Rev. B 59, 12398-418 (1999).

[29] F. Lesage and H. Saleur, Phys. Rev. Lett. 80, 4370 (1998).

[30] T. Fujisawa, T. H. Oosterkamp, W. G. van der Wiel, B. W. Broer, R. Aguado, S. Tarucha, L. P. Kouwenhoven, Science 282, 932 (1998).

[31] S. Tarucha, D. G. Austing, Y. Tokura, W. G. van der Wiel, and L. P. Kouwenhoven, Phys. Rev. Lett. 84, 2485 (2000).

[32] W.G. van der Wiel, S. De Franceschi, T. Fujisawa, J. M. Elzerman, S. Tarucha, L.P. Kouwenhoven, Science 289, 2105 (2000).

[33] S. Sasaki, S. De Franceschi, J. M. Elzerman, W. G. van der Wiel, M. Eto, S. Tarucha, L. P. Kouwenhoven, Nature 405, 764-767 (2000).

[34] T. Brandes, B. Kramer, Phys. Rev. Lett. 83, 3021 (1999).

[35] M. Keil, H. Schoeller, to be published.

[36] A. C. Hewson, The Kondo Problem to Heavy Fermions (Cambridge University Press, Cambridge, 1997).

[37] P. Coleman, C. Hooley, O. Parcollet, Phys. Rev Lett. 86, 4088 (2001).

[38] A. Rosch, J. Kroha, P. Wölfle, Phys. Rev. Lett. 87, 156802 (2001).

[39] Yu-Wen Lee, Yu-Li Lee, cond-mat/0105009

[40] D. Giuliano, B. Jouault, A. Tagliacozzo, cond-mat/0101341

[41] E. Lebanon, A. Schiller, cond-mat/0105488. 
[42] A. Kaminski, Y. V. Nazarov, and L. I. Glazman, Phys. Rev. B 62, 8154 (2000).

[43] A. Kaminski, Y. V. Nazarov, and L. I. Glazman, Phys. Rev. Lett. 83, 384 (1999).

[44] V. D. Lakhno, Polarons and Applications (Wiley, Chichester, 1994).

[45] S. I. Pekar, V. I. Sheka, G. V. Dimitrenko, Sov. Phys. JETP 36, 771 (1973) [Zh. Eksp. Teor. Fiz. 63, 1455 (1972)].

[46] S. I. Pekar, L. S. Khazan, V. I. Sheka, Sov. Phys. JETP 38, 999 (1974) [Zh. Eksp. Teor. Fiz. 65, 1999 (1973)].

[47] C. Fürst, A. Leitenstorfer, A. Laubereau, and R. Zimmermann, Phys. Rev. Lett. 78, 3733 (1997).

[48] M. Keil, diploma thesis, University of Heidelberg, Germany (1998).

[49] P. Lenz, F. Wegner, Nuclear Physics B 482, 693 (1996).

[50] H. Kleinert, Path Integrals in Quantum Mechanics, Statistics and Polymer Physics (World Scientific, Singapore, 2nd edition, 1995).

[51] Y. Lu, R. Rosenfelder, Phys. Rev. B 46, 5211 (1992).

[52] S. K. Kehrein, A. Mielke, P. Neu, Z. Phys. B 99, 269 (1996).

[53] D. Chandler, in Liquids, Freezing, and the Glass Transition, edited D. Levesque et al. (Les Houches Lectures, Elsevier, Amsterdam, 1991).

[54] A. J. Leggett, in Percolation, Localization and Superconductivity, edited by M. Goldman, S. A. Wolf (vol. 109, NATO Advanced Study Institute, Series B: Physics, Plenum, New York, 1984).

[55] H. Grabert, H. Wipf, in Advances in Solid State Physics (vol. 30, Vieweg, Braunschweig, 1990), p. 1.

[56] B. Golding, N. M. Zimmerman, S. N. Coppersmith, Phys. Rev. Lett. 68, 998 (1992).

[57] J. T. Stockburger, C. H. Mak, Phys. Rev. Lett. 80, 2657 (1998).

[58] A. O. Caldeira, A. J. Leggett, Ann. Phys. (New York) 149, 374 (1983).

[59] M. Sassetti, U. Weiss, Phys. Rev. A 41, 5383 (1990).

[60] H. Shiba, Progr. Theor. Phys. 54, 967 (1975).

[61] C. P. Slichter, Principles of Magnetic Resonance (Harper \& Row, New York, 1963).

[62] R. P. Feynman, F. L. Vernon, Ann. Phys. (N.Y.) 24, 18 (1963). 
[63] M. Sassetti, U. Weiss, Phys. Rev. Lett. 65, 2262 (1990).

[64] R. Egger, C. H. Mak, Phys. Rev. B 50, 15210 (1994).

[65] J. T. Stockburger, Phys. Rev. E 59, R4709 (1999).

[66] S. K. Kehrein, A. Mielke, Ann. Physik (Leipzig) 6, 90 (1997).

[67] S. Charkravarty, Phys. Rev. Lett. 50, 1811 (1982).

[68] A. J. Bray, M. A. Moore, Phys. Rev. Lett. 49, 1546 (1982).

[69] V. Hakim, F. Guinea, A. Muramatsu, Phys. Rev. B 32, 4410 (1985).

[70] T. A. Costi, Phys. Rev. Lett. 80, 1038 (1998).

[71] T. A. Costi, Phys. Rev. B 55, 3003 (1997).

[72] D. Weinmann, diploma thesis, University of Stuttgart, Germany (1991).

[73] I. S. Gradshteyn, I. M. Ryzhik, Table of Integrals, Series, and Products (Academic Press, London, 1994).

[74] Private communication with J. T. Stockburger.

[75] A. Geist, A. Beguelin, J. Dongarra, W. Jiang, R. Manchek, V. Sunderam, PVM: Parallel Virtual Machine (MIT Press, Cambridge, 1994).

[76] M. Raikh, A. Asenov, Superlatt. Microstr. 11, 325 (1992).

[77] S. M. Cronenwett, T. H. Oosterkamp, L. P. Kouwenhoven, Science 281, 540 (1998).

[78] D. Loss and D. P. DiVincenzo, Phys. Rev. A 57, 120 (1998).

[79] T. H. Stoof and Y. V. Nazarov, Phys. Rev. B 53, 1050 (1996).

[80] Private communication with T. Fujisawa.

[81] Private communication with T. Brandes.

[82] H. Bruus, K. Flensberg, and H. Smith, Phys. Rev. B 48, 11144 (1993).

[83] J. Kondo, Prog. Theor. Phys. 32, 37 (1964).

[84] N. Andrei, Phys. Rev. Lett. 45, 379 (1980).

[85] P. B. Wiegmann, Sov. Phys. JETP Lett. 31, 392 (1980).

[86] H. A. Bethe, Z. Phys. 71, 205 (1931).

[87] D. Goldhaber-Gordon et al., Nature 391, 156 (1998). 
[88] F. Simmel, R. H. Blick, J. P. Kotthaus, W. Wegscheider, M. Bichler, Phys. Rev. Lett. 83, 804 (1999).

[89] J. Schmid, J. Weis, K. Eberl, K. v. Klitzing, Phys. Rev. Lett. 84, 5824 (2000).

[90] A. F. Nikiforov, V. B. Uvarov, Special Functions of Mathematical Physics (Birkhäuser Verlag, Basel, 1988).

[91] G. Yuval, P. W. Anderson, Phys. Rev. B 1, 1522 (1970).

[92] J. v. Delft, H. Schoeller, Ann. Phys. (Berlin) 7, 225-305 (1998).

[93] A. M. Tsvelik, P. B. Wiegmann, Adv. Phys. 32, 453 (1983).

[94] W. H. Press, S. A. Teukolsky, W. T. Vetterling, B. P. Flannery, Numerical Recipes in $C$ (Cambridge University Press, New York, 1992).

[95] J. R. Cash, A. H. Karp, ACM Transactions on Mathematical Software 16, 201 (1990). 



\section{Ackowledgements}

I would like to thank Prof. Dr. Kurt Schönhammer for giving me the opportunity to write this thesis and for his unconditional support. He always showed a great interest in my work and readily discussed the results with me. His comments have helped me very much. Furthermore, I am grateful to him for critically reading the manuscript of my thesis.

I am deeply indebted to Prof. Dr. Herbert Schoeller whose continual enthusiasm for my scientific work always encouraged me over the past few years. Our numerous discussions and his suggestions have been of great benefit to me. I very much enjoyed our collaboration which turned out to be very fruitful. Finally, I thank him for his kind hospitality during my several stays at the University of Karlsruhe, the Forschungszentrum Karlsruhe and the Rheinisch-Westfälische Technische Hochschule Aachen.

My special thanks go to Dr. Jürgen Holm for his patience concerning all kinds of computer problems. His advice was always very helpful. For their support of my computational work out of Göttingen I would also like to thank Dr. Matthias Hettler in Karlsruhe and Dr. Uwe Kahlert in Aachen.

I am also grateful to Prof. Dr. Ulrich Weiss, Dr. Jürgen Stockburger, Dr. Theo Costi, Dr. Tobias Brandes and Dr. Toshimasa Fujisawa for answering questions and/or for their numerical data.

I would like to thank Urs Wiesemann, Thomas Korb and Dr. Maarten Wegewijs for useful discussions and for their comments on the manuscript of my thesis.

Finally, I want to express my thanks to all of our present and former group members who contributed to the friendly atmosphere which I appreciated very much.

This work was financially supported by the Deutsche Forschungsgemeinschaft as part of the Sonderforschungsbereich 345 . 



\section{Lebenslauf}

Name:

Geburtsdatum:

Geburtsort:

Familienstand:

Staatsangehörigkeit:

Schulausbildung:

$1978-1980$

1980 - 1982

1982 - 1991

06/1991

Zivildienst:

07/1991 - 09/1992

Studium:

10/1992 - 09/1998

$06 / 1994$

02/1995 - 07/1995

$09 / 1998$

10/1995 - 07/1998

07/1998

\section{Promotion:}

seit 02/1999
Markus Keil

21.12.1971

Groß-Gerau

ledig

deutsch

Hasengrundschule, Rüsselsheim

Grundschule Selbecke, Hagen

Fichte-Gymnasium, Hagen

Allgemeine Hochschulreife

Zivildienst bei der Arbeiterwohlfahrt in Hagen

Studium der Physik

an der Ruprecht-Karls-Universität zu Heidelberg

Vordiplom in Physik

Studium an der University of Adelaide in Adelaide, Australien

Diplom in Physik

Diplomarbeit bei Prof. Dr. Franz Wegner:

„Untersuchung des Polarons mittels Flußgleichungen“

Studium der Volkswirtschaftslehre

an der Ruprecht-Karls-Universität zu Heidelberg

Vordiplom in Volkswirtschaftslehre

Wissenschaftlicher Mitarbeiter

am Institut für Theoretische Physik

der Georg-August-Universität zu Göttingen,

Promotion bei Prof. Dr. Kurt Schönhammer:

„Renormalization Group Theory

for Quantum Dissipative Systems in Nonequilibrium“ 
